\title{
ASSESSING UNCONSCIOUSNESS IN LIVESTOCK AT SLAUGHTER
}

Merel Verhoeven

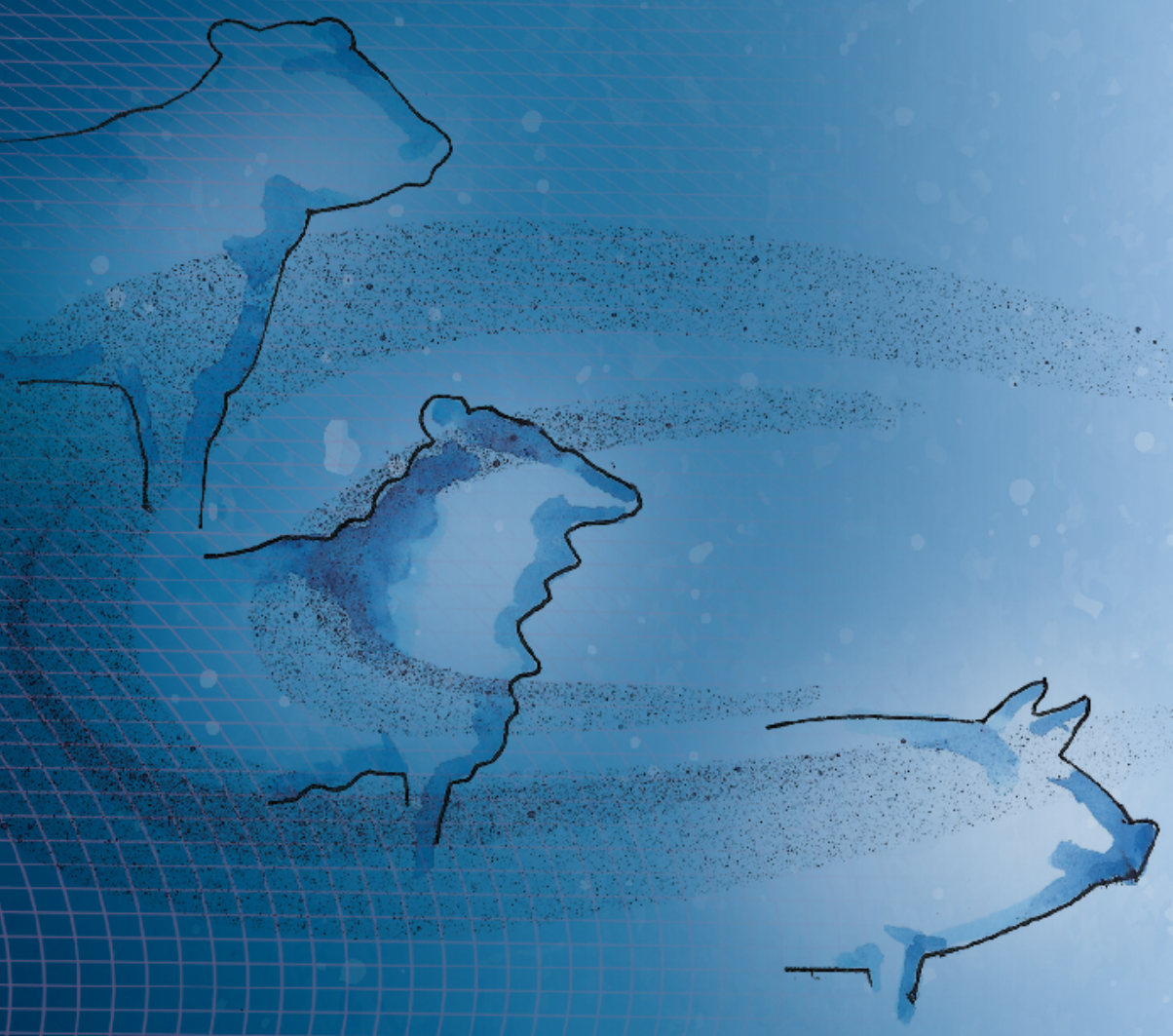





\section{ASSESSING UNCONSCIOUSNESS IN LIVESTOCK AT SLAUGHTER}

Merel Verhoeven 


\section{Thesis committee}

\section{Promotor}

Prof. Dr B. Kemp

Professor of Adaptation Physiology

Wageningen University

\section{Co-promotors}

Dr M.A. Gerritzen

Senior Scientist, Animal Welfare Group

Wageningen UR Livestock Research

Prof. Dr L.J. Hellebrekers

Director Central Veterinary Institute

Wageningen University and Research Centre

\section{Other members}

Prof. Dr P.W.G. Groot Koerkamp, Wageningen University

Dr D. McKeegan, University of Glasgow, United Kingdom

Dr E.M.C. Terlouw, l'Institut National de la Recherche Agronomique, France

Dr M. Marahrens, Friedrich-Loeffler-Institut, Celle, Germany

This research was conducted under the auspices of the Graduate School of Wageningen Institute of Animal Science (WIAS) 


\title{
ASSESSING UNCONSCIOUSNESS \\ IN LIVESTOCK AT SLAUGHTER
}

\author{
Merel Theresa Willemijn Verhoeven
}

\section{Thesis}

Submitted in fulfilment of the requirements for the degree of doctor at Wageningen University

by the authority of the Rector Magnificus

Prof. Dr A.P.J. Mol,

in the presence of the

Thesis Committee appointed by the Academic Board

to be defended in public

on Friday 11 November 2016

at 1.30 p.m. in the Aula. 
Merel Theresa Willemijn Verhoeven

Assessing unconsciousness in livestock at slaughter 188 pages.

$\mathrm{PhD}$ thesis, Wageningen University, Wageningen, NL (2016)

With references, with a summary in English

ISBN: 978-94-6257-906-4

DOI: $10.18174 / 388582$ 
Voor $p p$ \& $m m$ 



\section{ABSTRACT}

Verhoeven, M.T.W. (2016). Assessing unconsciousness in livestock at slaughter. $\mathrm{PhD}$ thesis, Wageningen University, The Netherlands

Assessing unconsciousness in livestock at different stages of the slaughter process is a legal requirement according to EU legislation. The assessment can be based on absence of readily observable indicators (behavioural indicators, physical signs and reflexes) or, under experimental conditions, by recording and subsequent assessment of brain activity as presented in an electroencephalogram (EEG). There is no consensus, however, to what extent different behavioural indicators, physical signs and reflexes accurately reflect unconsciousness. The aim of this thesis was to study the relationships between these readily observable indicators and EEG activity to provide information on 1. the exact point in time at which animals lose consciousness when subjected to different stunning and slaughter methods and 2. the validity of behavioural indicators, physical signs and reflexes used to assess unconsciousness at slaughter under commercial conditions. The results showed a large variation in time to loss of consciousness, based on EEG activity, both between stunning and slaughter procedures and amongst animals. Captive bolt stunned calves lost consciousness instantly following the stun. Loss of consciousness in pigs during $\mathrm{CO}_{2}$ stunning varied from 21 to $61 \mathrm{~s}$ after start of the exposure. An increased $\mathrm{CO}_{2}$ concentration decreased the time to loss of consciousness. Times to loss of consciousness in sheep slaughtered without stunning varied from 6 to $24 \mathrm{~s}$. In cattle slaughtered without stunning, times to loss of consciousness varied from $14 \mathrm{~s}$ up to over two minutes. Following captive bolt stunning in calves, absence of reflexes indicated unconsciousness. When consciousness was lost gradually (e.g. $\mathrm{CO}_{2}$ stunning and nonstunned slaughter) none of the readily observable indicators could identify the exact point in time at which animals lost consciousness. Absence of rhythmic breathing, corneal reflex- and eyelid reflex were valid indicators of unconsciousness following $\mathrm{CO}_{2}$ stunning and non-stunned slaughter, but these indicators were quite conservative as they were initially absent long after EEG activity indicated unconsciousness. When presence of these indicators would require (re)stunning, many animals will have to be (re)stunned. The results also showed that under full commercial conditions, stunning effectiveness must be closely monitored by slaughter plant employees, since risks for stun failures are higher under commercial conditions compared to experimental conditions. 



\section{CONTENTS}

CHAPTER 1 GENERAL INTRODUCTION

CHAPTER 2 INDICATORS USED IN LIVESTOCK TO ASSESS

UNCONSCIOUSNESS AFTER STUNNING: A REVIEW

CHAPTER 3 VALIDATION OF BEHAVIOURAL INDICATORS USED TO ASSESS UNCONSCIOUSNESS IN SHEEP

CHAPTER 4 VALIDATION OF BEHAVIOURAL INDICATORS USED TO ASSESS UNCONSCIOUSNESS IN VEAL CALVES AT SLAUGHTER

CHAPTER 5 TIME TO LOSS OF CONSCIOUSNESS AND ITS RELATION TO 85 BEHAVIOUR IN SLAUGHTER PIGS DURING STUNNING WITH 80\% OR 95\% CARBON DIOXIDE

CHAPTER 6 SCIENTIFIC CONTRIBUTION TO THE ETHICAL DEBATE ON 105 STUNNING AND SLAUGHTER OF LIVESTOCK

CHAPTER 7 GENERAL DISCUSSION 119

$\begin{array}{ll}\text { APPENDICES } & \\ \text { SUMMARY } & 146 \\ \text { REFERENCES } & 150 \\ \text { LIST OF PUBLICATIONS } & 172 \\ \text { ACKNOWLEDGEMENTS } & 176 \\ \text { ABOUT THE AUTHOR } & 182 \\ \text { TRAINING AND SUPERVISION PLAN } & 184 \\ \text { COLOPHON } & 188\end{array}$



GENERAL INTRODUCTION 


\section{INTRODUCTION}

In 2014, 536 million broilers, 14 million fattening pigs, 2 million cattle and nearly 1 million sheep were slaughtered in The Netherlands (CBS, 2014). The slaughter process itself consists of two stages: stunning and bleeding. Stunning refers to 'any intentionally induced process which causes loss of consciousness and sensibility without pain, including any process resulting in instantaneous death' (Council Regulation (EC) 1099/2009). Bleeding follows when an animal's throat is cut, using a very sharp knife, severing the major blood vessels in the animal's neck that supply the brain, ensuring rapid blood loss and eventually resulting in death of the animal (EFSA, 2004).

It is generally acknowledged that slaughter by neck incision of conscious animals causes pain and that bleeding while conscious may distress the animal (Gregory, 2005a; Johnson et al., 2012; Johnson et al., 2015). European legislation therefore states that animals should be stunned before bleeding, with exception of animals subject to particular methods of slaughter prescribed by religious rites (Council Regulation (EC) 1099/2009). European legislation also states that regular checks should be carried out to ensure that animals do not regain consciousness between the end of the stunning process and death, since unconscious animals do not experience pain or distress. In animals subjected to particular methods of slaughter prescribed by religious rites, unconsciousness must be ascertained before release from restraint (Council Regulation (EC) 1099/2009). For all stunning and slaughter methods it is thus critical to determine the time to loss of consciousness and duration of unconsciousness.

Assessing unconsciousness is generally performed by evaluation of behavioural indicators (e.g. vocalisations and coordinating walking movements), physical signs (e.g. rhythmic breathing and loss of posture), testing of reflexes (e.g. eyelid-, and corneal reflex) and assessment of brain activity, as presented in an electroencephalogram (EEG). The use of EEG recordings is considered the most objective way to assess unconsciousness, but can only be applied under experimental conditions. The European Food Safety Authority (EFSA) has therefore stipulated that EEG recordings should be used in research (performed under experimental conditions) that evaluates stunning interventions to establish relations between EEG activity and commonly used indicators applied under commercial conditions (EFSA, 2013a). There is, however, a lack of studies that establish these relationships. When more information could be obtained about unambiguous and quick measures to validly assess unconsciousness at slaughter, practical recommendations could be formulated so that professionals involved in the slaughter process can reliably assess unconsciousness during the slaughter process (Erasmus et al., 2010a).

The main aim of this thesis was to investigate times to loss of consciousness and the relationships between behavioural indicators, physical signs and reflexes and EEG activity in different livestock species subjected to different stunning and slaughter methods. 


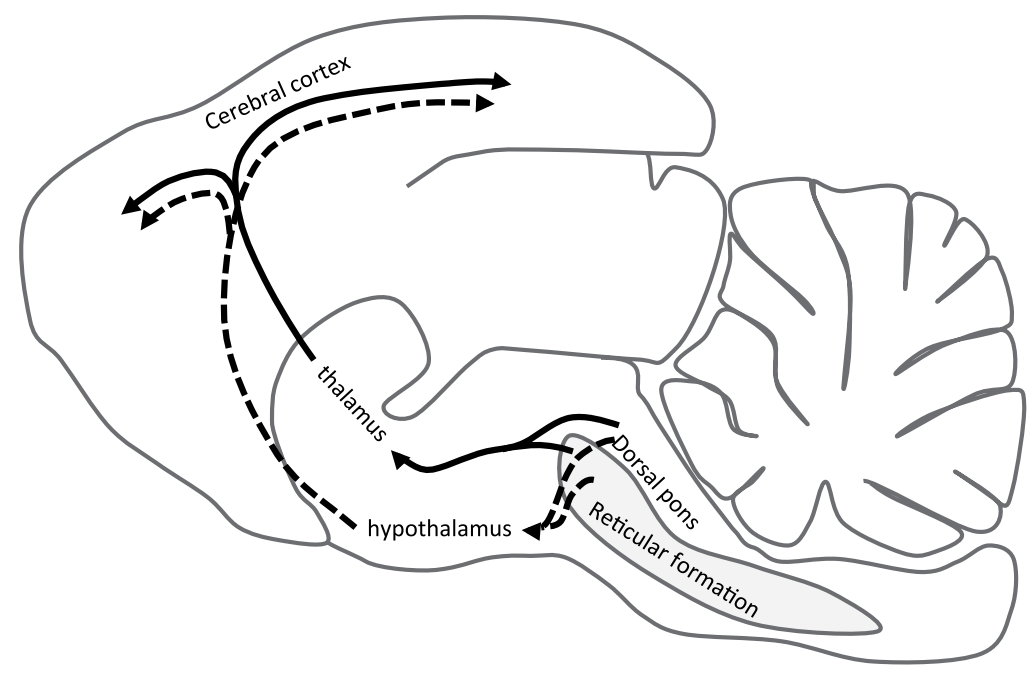

FIGURE 1

Dorsal (continuous arrows) and ventral pathways (dotted arrows) of the ascending reticular activating system arising from the reticular formation and the dorsal pons. ${ }^{1}$

\section{(UN)CONSCIOUSNESS}

Consciousness is a multifaceted concept, which has been broadly defined in many different ways. In clinical neurological examination consciousness is 'generally equated with the waking state, and the abilities to perceive, interact and communicate with the environment and with others' (Zeman, 2001). Consciousness itself has two distinct dimensions: arousal, or wakefulness (i.e. level of consciousness and awareness (i.e. content of consciousness) (Zeman, 2005). When a subject is awake, this subject will usually also be aware. During the slaughter process, an animal will be aware of its environment and this awareness is related to functional primary and associative cortices. The primary cortices receive direct (e.g. visual, auditory or somatosensory) input and are directly involved in execution of movements. Associative cortices are cortices outside the primary areas, that are involved in the complex processing input from primary cortices and the subsequent generation of behaviour. Earlier studies have indicated that neural activity in primary cortices alone is not sufficient for awareness, but that connection to associative cortices is needed as well (Crick and Koch, 1995).

\footnotetext{
${ }^{1}$ Reprinted from Meat Science, 118 , Terlouw, C., Bourguet, C. and Deiss, V. Consciousness, unconsciousness and death in the context of slaughter. Part I. Neurobiological mechanisms underlying stunning and killing, $p$ 133-146, Copyright (2016), with permission from Elsevier.
} 
Wakefulness is a state of consciousness. Consciousness in this sense is not binary, but a matter of degree: there are different states of consciousness from waking through sleep into coma and a person can be wide awake or half-awake (Zeman, 2001; Zeman, 2005). Wakefulness is a functional brain state that allows the performance of several 'high brain functions', resulting in diverse behavioural, cognitive and emotional activities (Lin, 2000). A network of nerve fibres in the brainstem and specific structures in the dorsal pons modulate wakefulness by ascending projections to the cerebral cortex. These projections are called the 'Ascending Reticular Activating System' (ARAS) (Brown et al., 2012) and are composed of several neuronal circuits connecting the brainstem to the cortex. These neuronal connections originate mainly in the reticular formation of the brainstem and project through synaptic relays in the intralaminar nucleus of thalamus to the cerebral cortex (Brown et al., 2012). Figure 1 shows the two major pathways for this system. One, the dorsal pathway, passes the thalamus and projects thereafter on the cortex. The ventral pathway runs, amongst others, via the hypothalamus before projecting onto the cortex (Brown et al., 2012; Terlouw et al., 2016a). An animal that is asleep can potentially be awakened by stimuli that activate the ARAS which in turn activates the neocortex to produce wakefulness and consciousness (Klemm, 2011). If the reticular formation or ARAS is damaged due to stunning, the cortex does not function fully or insufficiently and the subject is unconscious (Terlouw et al., 2016a). It has been generally accepted that humans possess consciousness based upon their ability to communicate experiences and emotions and the fact that they are aware of themselves. Nowadays, the general opinion is that non-human animals can also be in different conscious states and that they have the capacity to exhibit intentional behaviours (Low et al., 2012).

The opposite state of consciousness, i.e. unconsciousness, is defined as: 'a state of unawareness (loss of consciousness) in which there is temporary or permanent disruption to brain function. As a consequence of this disruption the unconscious animal is unable to respond to normal stimuli, including pain' (EFSA, 2006). In addition, it is believed that an unconscious animal should not be able to perceive normal stimuli, including pain. Otherwise, a conscious, yet immobilized animal would be considered unconscious based on the EFSA definition.

\section{(UN)CONSCIOUSNESS IN RELATION TO DIFFERENT STUNNING METHODS}

Disruption of brain function at slaughter can occur as a result of different stunning methods employed that include mechanical stunning, electrical stunning and gas stunning. Figure 2 represents a schematic overview of the most commonly used stunning methods on different livestock species at commercial slaughter plants in the EU. The different stunning methods are shortly described below. 


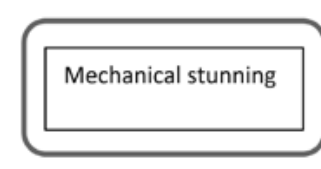

Penetrating captive-bolt stunning

Non-penetrating captive-bolt stunning

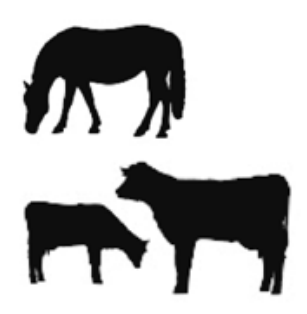

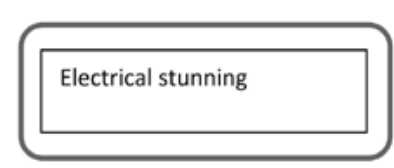

Head-only stunning

Head-to-body stunning

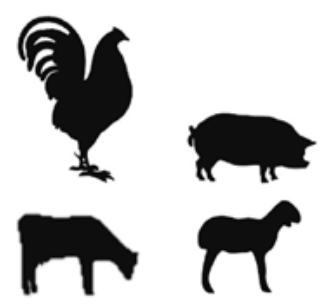

Gas stunning

Carbon dioxide $\left(\mathrm{CO}_{2}\right)$ stunning

Inert (Argon, Nitrogen) gas stunning

\section{FIGURE 2}

Schematic representation of the most commonly used stunning methods at commercial slaughter plants in the EU.

\section{Mechanical stunning}

One of the ways by which mainly larger animals, such as cattle and horses, are stunned instantaneously is by means of a penetrating captive bolt, also referred to as mechanical stunning. With penetrating captive bolt stunning, a metal rod is forced through the skull, damaging (part of) the brain. A sufficient force will cause intracranial trauma in a way that organized electrical activity of the brain is disrupted. The duration of unconsciousness depends on how much damage is done to nervous tissue and to which degree the blood supply is reduced (Shaw, 2002). Non-penetrating captive bolt stunning consists of firing a mushroom-headed blunt bolt on the forehead of animals (under $10 \mathrm{~kg}$ ) (Council Regulation (EC) 1099/2009). The skull of the animal may be fractured, but the bolt will not penetrate through the skull (EFSA, 2004).

\section{Electrical stunning}

Electrical stunning is another stunning method that induces unconsciousness instantly following the stun. Electrical stunning methodology is based upon passing an electrical current through the animal brain leading to a so-called epileptiform seizure. The epileptiform seizure is the result of continuous synchronised depolarisation or hyperpolarizing of many neurons (Carlson, 2007). Two frequently applied electrical stunning methods are head-only stunning and head-to-body stunning. In the case of 
head-to-body stunning, a current is not only applied to the head, but also to the heart to induce fibrillation or the stopping of the heart. The duration of unconsciousness depends on the amount of the current that passes through the brain and the exposure time to the current (Anil and McKinstry, 1998).

\section{Gas stunning}

Carbon dioxide $\left(\mathrm{CO}_{2}\right)$ is a commonly used gas for stunning pigs and poultry in the EU. Pigs and poultry are immersed, often in groups, into a pit, tunnel or chamber that is pre-filled with $\mathrm{CO}_{2}$. Exposure to high concentrations of $\mathrm{CO}_{2}$ will induce a state of hypercapnic hypoxia and is associated with changes in blood parameters such as a lowered pH (Martoft et al., 2002). Because $\mathrm{CO}_{2}$ travels across the blood-brain barrier relatively easy, the high $\mathrm{CO}_{2}$ levels cause rapid acidification of the cerebrospinal fluid. The acidification of the brain cells induces a depression of brain activity that causes loss of consciousness and ultimately death (Martoft et al., 2002).

Loss of consciousness is not immediate upon exposure to high $\mathrm{CO}_{2}$ levels, but depends on the $\mathrm{CO}_{2}$ concentration used and the speed at which animals are immersed into the highest concentration of $\mathrm{CO}_{2}$ at the bottom of the well (Troeger, 1991, Raj and Gregory, 1996). Time to unconsciousness has been reported 14-60 s after initial exposure to 80$90 \% \mathrm{CO}_{2}$ (Raj and Gregory, 1996; EFSA, 2004; Rodriguez et al., 2008). The duration of unconsciousness depends on the concentration of $\mathrm{CO}_{2}$ used and the exposure time to the gas (EFSA, 2004).

\section{Slaughter without stunning}

Livestock must be stunned prior to bleeding as stated by European legislation. Slaughter prescribed by religious rites, however, is exempt from this requirement (Council Regulation (EC) 1099/2009). During this so-called non-stunned slaughter, animals are restrained and bled by a transverse incision of the neck, severing the skin, muscles, trachea, oesophagus, carotid arteries, jugular veins and major nerves. Bleeding of the animal will result in unconsciousness, eventually followed by death. Consciousness in sheep and poultry is generally lost around $15 \mathrm{~s}$ and 20-35 s, respectively after the neck cut, with little variation between animals (Gregory and Wotton, 1984; Devine et al., 1986; Gregory and Wotton, 1986; Terlouw et al., 2016b).

Results from studies on non-stunned slaughter of cattle show considerable more variation in time to loss of consciousness compared to sheep and poultry. Some studies suggest a rapid loss of consciousness (4-7 s) following the neck cut (Nangeroni and Kennett, 1963; Bager et al., 1992). Other studies report loss of consciousness between 34 and $85 \mathrm{~s}$, but also the possibility of an intermittent return to consciousness for up to 123 to $323 \mathrm{~s}$ after the neck cut (Newhook and Blackmore, 1982b; Bager et al., 1992; Lambooij et al., 2012). Prolonged consciousness in cattle is believed to be caused by two phenomena. First, the 
vertebral artery stays intact in bovine when the neck is cut and continues to supply blood to the brain (Daly et al., 1988). Secondly, occlusion of severed arteries may cause impaired bleeding in cattle and therefore prolonged consciousness (Gregory et al., 2006; Gregory et al., 2010).

\section{METHODS TO ASSESS UNCONSCIOUSNESS AT SLAUGHTER}

Unconsciousness and thus the effectiveness of the stun can be assessed under commercial conditions by observing behavioural indicators, physical signs and reflexes. Under laboratory or experimental conditions, recording and observing changes in brain activity, as presented in an electroencephalogram (EEG), can be employed to this aim (Alkire et al., 2008; Verhoeven et al., 2015a).

\section{Electroencephalogram (EEG)}

The EEG displays the electrical potentials produced by nerve cells in the cerebral cortex recorded via electrodes attached at various locations on the scalp (Knudsen, 2005) (Figure 3). There are four different types of wave patterns in the EEG that can be distinguished based on their respective frequencies and that are related to the state of consciousness: delta- $(\delta ;<4 \mathrm{~Hz})$, theta- $(\theta ; 4$ to $8 \mathrm{~Hz})$, alpha- $(\alpha ; 8$ to $12 \mathrm{~Hz})$ and beta- $(\beta ;>12 \mathrm{~Hz})$ waves (Figure 4).

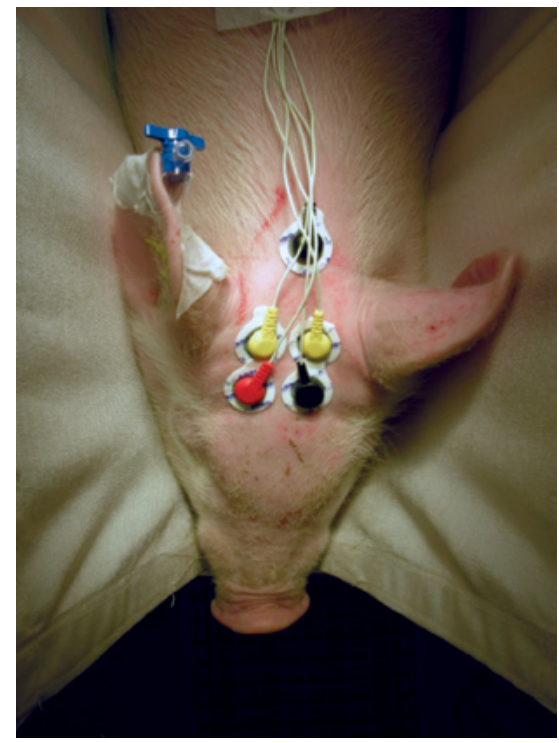

FIGURE 3

Example of a pig equipped with electrodes to measure the EEG.
Alpha $(\alpha)$ : 8-15 Hz

Beta $(\beta): 16-31 \mathrm{~Hz}$

Theta $(\theta): 4-7 \mathrm{~Hz}$

Delta $(\delta):<4 \mathrm{~Hz}$

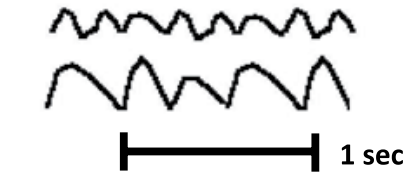

\section{FIGURE 4}

Example of alpha-, beta-, theta-, and deltaelectroencephalography frequencies. 
Both $\delta$ - and $\theta$ - (slow wave) waves are related to sleep or reduced consciousness. Alphawaves are prominent in subjects that are conscious, but mentally inactive (closing eyes and relaxation) and $\beta$-waves are associated with active movements and increased alertness (Kooi et al., 1978; Schomer and Da Silva, 2012).

When an animal loses consciousness, changes from an EEG pattern typically observed in an awake animal can be seen (Sandercock et al., 2014) (Figure 5). The EEG pattern observed in an awake animal is characterised by low-amplitude, high-frequency waves $(\geq 8 \mathrm{~Hz}$ ) that are unsynchronised because of irregularly firing neurons (Baars et al., 2003) (Figure 5a). Unconsciousness on the other hand is characterised by high amplitude, lowfrequency waves $(\leq 8 \mathrm{~Hz}$ ) caused by synchronised firing of neurons (Baars et al., 2003) (Figure 5b).

When stunning causes irreversible brain damage, the EEG pattern continues to change until a flat line is observed, also known as an iso-electric EEG (Figure 5c). This indicates that all brain activity has ceased (Gibson et al., 2007; McKeegan et al., 2007; Kroeger et al., 2013). The exact time of onset of unconsciousness, based solely on EEG activity, is difficult to determine as changes are often gradual. The iso-electric EEG, however, is never compatible with consciousness.

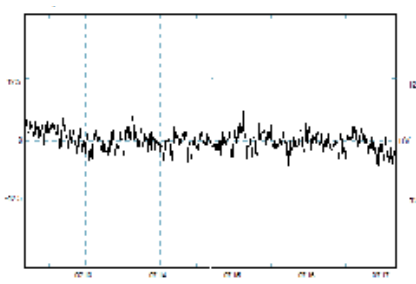

A

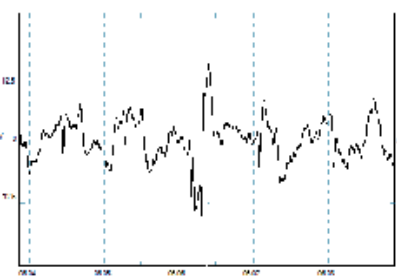

B

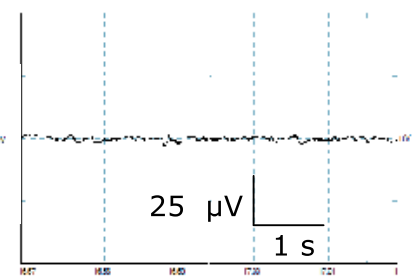

C

\section{FIGURE 5A-C}

Representative examples of the different stages identified with visual assessment of brain activity, presented in an electroencephalogram (EEG), prior to and after $\mathrm{CO}_{2}$ stunning in pigs. The three stages from left to right: baseline (a), unconscious (b), and iso-electric (c). Total X-axis represents 5 seconds, Y-axis represents amplitude of the EEG-trace $(\mu \mathrm{V})$.

\section{Fast Fourier Transformation}

Next to visual assessment of the EEG, as described previously, raw EEG data can also be analysed by computing a Fast Fourier Transformation (FFT). With FFT analyses, the frequency composition of the signal is determined, or alternatively formulated, it provides information on how much power is present in the different frequency bands. Further (automatic) calculations of the FFT can lead to EEG derivatives presented as a single value or percentage. Examples of such derivates include: the total power (Ptot), which is the area underneath the frequency spectrum curve, the median frequency (F50), which 
is the frequency below which $50 \%$ of the power is located and the spectral edge frequency (F95), which is the frequency below which 95\% of the power is located. Figure 6 shows an example of a frequency spectrum from a two second EEG epoch, demonstrating the derived values for total power (PTOT), median frequency (F50), and the spectral edge (F95). These readout parameters are considered useful and standardized quantitative tools to uniformly describe changes in EEG activity (Murrell and Johnson, 2006).
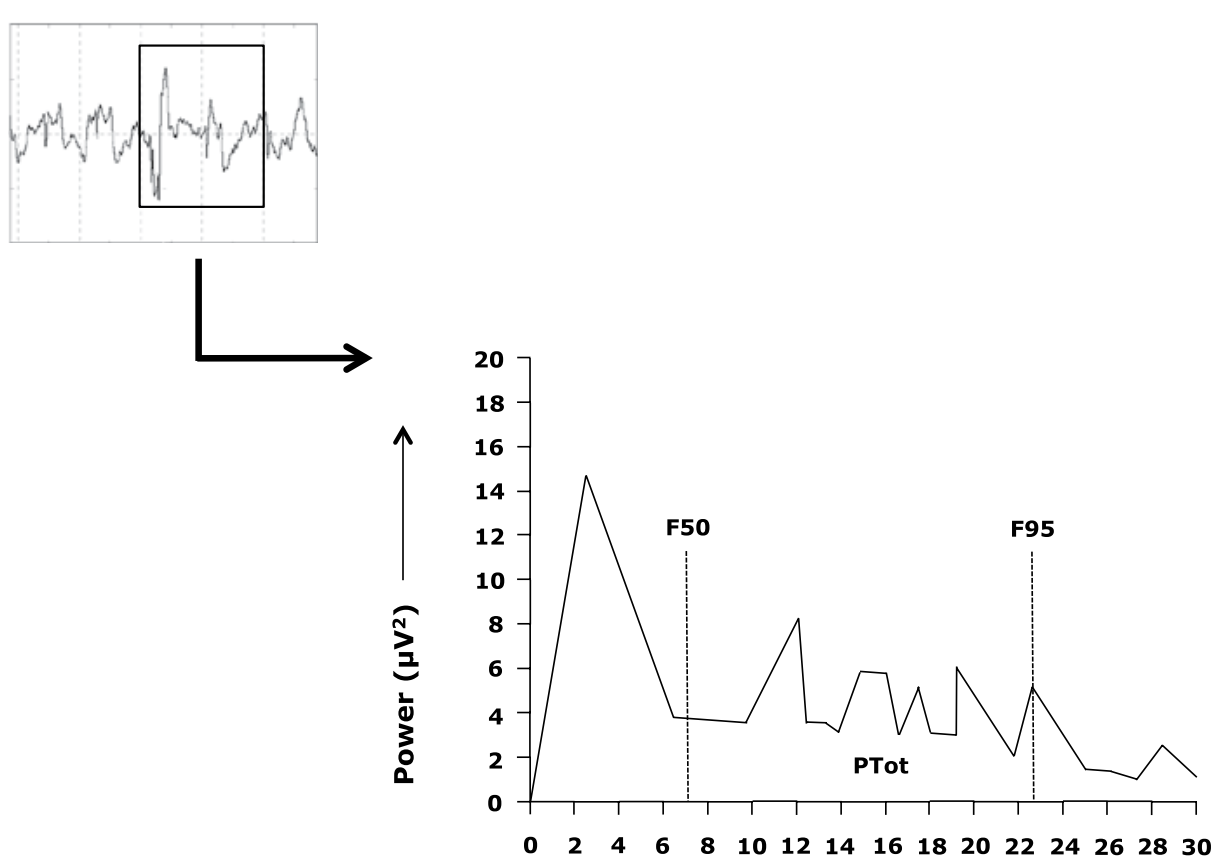

Frequency (Hz)

FIGURE 6

An example of a frequency spectrum from a two second EEG epoch, demonstrating the derived values for total power (PTOT), median frequency (F50), and the spectral edge (F95).

\section{Evoked potentials}

The EEG recording is also used to assess unconsciousness by way of generating evoked responses. Evoked responses are responses in the EEG following external stimuli (visual, somatosensory or auditory), generated in specific areas of the cerebral cortex, mid brain and brainstem compared to the ongoing spontaneous EEG signal (Gregory and Wotton, 1990; Knudsen, 2005). These responses occur at a fixed time after the stimulus has been applied and can therefore be extracted from the EEG (Knudsen, 2005). 


\section{Difficulties in the use of EEG recordings}

An important reason why the EEG is not used to assess unconsciousness in slaughter plants is that the method is expensive, time-consuming and requires a high degree of expertise compared to observing behavioural indicators, physical signs and reflexes. Another important reason is that the EEG is easily disturbed by artefacts that are animal related (eye or muscle movements) or technical related (cable movements, impedance fluctuation or $50 / 60 \mathrm{~Hz}$ interference) (Teplan, 2002). Experimental controlled situations provide a significantly better environment to limit these artefact sources than commercial slaughter plants and EEG recordings are therefore currently only used under experimentalor semi-commercial conditions.

\section{INDICATORS USED AT COMMERCIAL SLAUGHTER PLANTS}

In commercial slaughter plants, unconsciousness is generally assessed by observing behavioural indicators, physical signs and reflexes. A questionnaire from Gerritzen and Hindle (2009) to experts in the field of stunning and killing showed there is much variation in how different indicators are valued and that this is partially due to the different stunning methods used (EFSA, 2004; Gerritzen and Hindle, 2009; Verhoeven et al., 2015a). Below, some of the most commonly used behavioural indicators, physical signs and reflexes applied in commercial slaughter plants are described.

\section{Behavioural indicators}

Cognitive responses and voluntary motor activity, such as coordinated walking movements, should be absent after stunning, since effective stunning induces a general dysfunction of the brain (Terlouw et al., 2016b). Vocalisation is also a conscious response, often following a stressful event and should therefore be absent after stunning (Grandin, 1998).

\section{Physical signs}

Absence of rhythmic breathing is a highly valued indicator for unconsciousness by experts (Gerritzen and Hindle, 2009). According to Grandin (2013) rhythmic breathing should be absent following a successful stun and rhythmic breathing after stunning is an indication to re-stun the animal immediately.

Loss of posture, i.e. the inability to remain in a standing position, is another highly valued indicator of unconsciousness. Control of a standing posture is regulated by the reticular formation and when this is damaged, the animal is not able to maintain such a posture. A conscious animal, on the other hand, may lift its head or body to regain its normal position (Anil, 1991). 


\section{Reflexes}

The absence of eye reflexes (i.e. corneal-, eyelid- and pupillary light reflex) are indicators of unconsciousness that are linked to functioning of the brain stem. The corneal reflex causes involuntary blinking of the eyelids in response to stimulation of the cornea. The eyelid or palpebral reflex also results in blinking as a response to touching the medial canthus of the eye and the pupillary light reflex is tested by letting light fall on the eye and observing whether the pupil adapts to it. The withdrawal reflex is elicited by applying a painful stimulus to the animal, such as a nose- or ear prick. In a survey on expert opinions, some of the eye reflexes and the withdrawal reflex were ranked high, and thus valued highly, as indicators for unconsciousness after all types of stunning (Gerritzen and Hindle, 2009).

\section{Relationships between different indicators}

Relationships between behavioural indicators, physical signs, reflexes and brain activity can increase our understanding to what extent different indicators reflect (un)consciousness. So far, there is a scarcity of studies that have looked into these relationships. Further, such research is often difficult to compare and extrapolate across species and different stunning and killing methods. Benson et al. (2012) looked into the relationship between loss of posture and the $\alpha / \delta$ ratio extracted from the EEG to assess loss of consciousness in broilers. The two methods were highly correlated and provided equal times in time to loss of consciousness. Studies as performed by Benson et al. (2012) provide additional, more objective data to support the use of behavioural indicators as a measure of unconsciousness and provide details when certain behaviours may be present or absent in an animal that loses consciousness during the slaughter process.

\section{AIM AND OUTLINE OF THE THESIS}

The aim of this thesis was to investigate times to loss of consciousness and the relationships between behavioural indicators, physical signs, reflexes and brain activity, as presented in an EEG, in different species subjected to different stunning and slaughter methods to find accurate indicators for unconsciousness that can be used under commercial conditions. First, a literature study (Chapter 2) was conducted to look into the different ways in which unconsciousness can be assessed after stunning. Since the assessment of unconsciousness can be performed in a variety of ways, the pros and cons to each assessment method were also reviewed. The review provided a good basis for the set-up of the multiple studies that were conducted hereafter and that are described in this thesis. In the first experiment described in Chapter 3, a selection of the indicators as described in Chapter 2 (i.e. 
eyelid-, withdrawal- and threat reflex and rhythmic breathing) was assessed in sheep during propofol anaesthesia. Anaesthetic agents can be used to induce different stages of unconsciousness and allow for recovery, as opposed to stunning and bleeding. The use of specific anaesthetic agents can therefore provide a methodology to validate the absence and presence of different indicators in the assessment of unconsciousness, and provide detailed information on relationships between these indicators and EEG activity. In the second experiment described in Chapter 3, sheep were subjected to non-stunned slaughter while assessing the same indicators as during propofol anaesthesia in relation to EEG activity. The results from that study indicated that the absence of certain reflexes at slaughter do not necessarily indicate unconsciousness and the study was repeated in veal calves (Chapter 4). The objective of Chapter 4 was to assess absence and presence of the threat-, withdrawal-, corneal-, and eyelid reflex and determine their relation with (un)consciousness as identified by EEG activity in both stunned and non-stunned slaughtered calves. A stunning method during which consciousness is not lost instantly is $\mathrm{CO}_{2}$ stunning. The prolonged time to loss of consciousness, however, is associated with aversive behaviour including excitements, escape- and retreat attempts and respiratory changes. In Chapter 5 relationships between behavioural measurements and onset of unconsciousness as identified by EEG activity during $80 \%$ or $95 \% \mathrm{CO}_{2}$ stunning of pigs was assessed.

From all chapters followed that the process of slaughtering raises many questions both from a technical as well as a societal perspective. This entails that the process of slaughter can be conceived as a moral dilemma, i.e. as a practice that requires further ethical reflection. Therefore, the aim of Chapter $\mathbf{6}$ was to map and explicate some of the ethical issues related to stunning and slaughter of livestock in the light of the scientific research performed in this thesis. In Chapter 7, the general discussion, the major findings of all studies described in this thesis are discussed. 
GENERAL INTRODUCTION | 23 
Animal (2015) 9: 320-330

DOI: $10.1017 / S 1751731114002596$

M.T.W. Verhoeven ${ }^{1,2}$, M.A. Gerritzen ${ }^{1}$, L.J. Hellebrekers ${ }^{3}$ and B. Kemp ${ }^{2}$

${ }^{1}$ Wageningen University and Research Centre, Livestock Research, PO Box 65, 8200 AB Lelystad, The Netherlands

${ }^{2}$ Adaptation Physiology Group, Department of Animal Sciences, Wageningen University, P.O. Box 338, 6700 AH Wageningen, The Netherlands

${ }^{3}$ Faculty of Veterinary Medicine, Utrecht University, PO Box 80154, 3508 TD Utrecht, The Netherlands 
INDICATORS USED IN LIVESTOCK TO ASSESS UNCONSCIOUSNESS AFTER STUNNING: A REVIEW 


\section{ABSTRACT}

Assessing unconsciousness is important to safeguard animal welfare shortly after stunning at the slaughter plant. Indicators that can be visually evaluated are most often used when assessing unconsciousness, as they can be easily applied in slaughter plants. These indicators include reflexes originating from the brain stem (e.g. eye reflexes) or from the spinal cord (e.g. pedal reflex) and behavioural indicators such as loss of posture, vocalisations and rhythmic breathing. When physically stunning an animal, for example, captive bolt, most important indicators looked at are posture, righting reflex, rhythmic breathing and the corneal or palpebral reflex that should all be absent if the animal is unconscious. Spinal reflexes are difficult as a measure of unconsciousness with this type of stunning, as they may occur more vigorous. For stunning methods that do not physically destroy the brain, for example electrical and gas stunning, most important indicators looked at are posture, righting reflex, natural blinking response, rhythmic breathing, vocalisations and focused eye movement that should all be absent if the animal is unconscious. Brain stem reflexes such as the corneal reflex are difficult as measures of unconsciousness in electrically stunned animals, as they may reflect residual brain stem activity and not necessarily consciousness. Under commercial conditions, none of the indicators mentioned above should be used as a single indicator to determine unconsciousness after stunning. Multiple indicators should be used to determine unconsciousness and sufficient time should be left for the animal to die following exsanguination before starting invasive dressing procedures such as scalding or skinning. The recording and subsequent assessment of brain activity, as presented in an electroencephalogram (EEG), is considered the most objective way to assess unconsciousness compared with reflexes and behavioural indicators, but is only applied in experimental set-ups. Studies performed in an experimental set-up have often looked at either the EEG or reflexes and behavioural indicators and there is a scarcity of studies that correlate these different readout parameters. It is recommended to study these correlations in more detail to investigate the validity of reflexes and behavioural indicators and to accurately determine the point in time at which the animal loses consciousness.

\section{IMPLICATIONS}

This review evaluates the different ways in which unconsciousness after stunning is assessed and weighs the pros and cons of these methods. Assessing unconsciousness is performed in a variety of ways, depending on species as well as the method of stunning. Assessing brain activity by way of electroencephalogram (EEG) analysis is suggested to be the most objective method to evaluate unconsciousness, but this is only applied in 
experimental set-ups. Studies in which correlations between the EEG and other indicators are looked at in more detail could provide additional information on the exact time points at which animals lose consciousness after stunning.

\section{INTRODUCTION}

European legislation provides laws, rules and procedures regarding the slaughter of livestock (GWvD, 1992; Council Directive 93/119/EC, 1993; Council Regulation (EC) 1999, 2009). Article 4 of Council Regulation (EC) 1099 (2009) describes the mandatory pre-slaughter stunning, with exception of particular methods of slaughter prescribed by religious rites, to ensure unconsciousness and insensibility to prevent unnecessary suffering of animals. There is no consensus about the extent to which slaughter of conscious, meaning sensible and/or aware, animals causes them pain and distress. It is claimed that when a clean incision is made with an exquisitely sharp knife, significant pain and distress are avoided (e.g. Grandin, 1994; Rosen, 2004). Johnson et al. (2012) suggest that massive stimulation of all sensory nerves after the neck cut may lead to shock and distress that would be experienced as pain for the duration of consciousness. Until now, neurophysiological methodology has not provided the ultimate answer to this issue. Because animals are considered not to experience pain when unconscious, it is important to validly determine unconsciousness after stunning. Stunning methods most frequently applied include mechanical stunning (captive bolt), applying an electrical current through the head of the animal or by immersion in a mixture of gasses consisting of (low level) oxygen $\left(\mathrm{O}_{2}\right)$, carbon dioxide $\left(\mathrm{CO}_{2}\right)$, argon $(\mathrm{Ar})$ and/or nitrogen $\left(\mathrm{N}_{2}\right)$. For all stunning methods, it is critical to determine the onset and duration of unconsciousness. Available data from different livestock species to examine the different methods used to assess unconsciousness, include reflexes and behavioural indicators. Less used in practice, but considered the most objective method for the assessment of unconsciousness, involves the evaluation of brain activity as presented in an electroencephalogram (EEG). The possibilities and limitations of the use of EEG for this purpose are further elaborated upon in this manuscript.

\section{CONSCIOUSNESS AND UNCONSCIOUSNESS}

Consciousness is defined in many different ways, but in general is associated with the awake state and the ability to perceive, interact and communicate with the environment and others (Zeman, 2001). The opposite state, that is, unconsciousness, is defined as: 'a state of unawareness (loss of consciousness) in which there is temporary or permanent disruption to brain function. As a consequence of this disruption the unconscious animal 
is unable to respond to normal stimuli, including pain' (EFSA, 2006). Disruption of brain function can occur as a result of brain concussion, administration of anaesthetics, anoxia or an electroconvulsive shock (Lopes da Silva, 1982). Some authors prefer the term insensibility over unconsciousness, as they find it less anthropomorphic (Blackmore and Delany, 1988). Insensibility refers to the complete inability to experience any sensations, including unpleasant sensations such as pain (Hemsworth et al., 2009). Pain is defined as 'an unpleasant sensory and/or emotional experience associated with actual or potential tissue damage, or described in terms of such damage' (Merskey, 1986). Pain is considered a conscious experience and needs to be avoided during the slaughter process. The term unconsciousness, as used in this review, also includes insensibility. Stunning of animals aims at inducing unconsciousness and thus insensibility, which lasts until the animal is dead. An animal is considered dead when: 'respiration and blood circulation have ceased as the respiratory and circulatory centres in the medulla oblongata are irreversibly inactive. Due to the permanent absence of nutrients and oxygen in the brain, consciousness is irreversibly lost' (EFSA, 2004). During the slaughter process, regular checks should be carried out to ensure that the animal does not present any signs of consciousness or sensibility in the period between the end of the stunning process and death (Council Regulation (EC) 1099, 2009).

Brain regions that are involved in consciousness are the cerebral cortex and thalamus, together forming the thalamocortical complex, which is regulated by the brainstem. A well-functioning brainstem and thalamus are essential for the maintenance of consciousness and damage to (one of) these regions can cause rapid loss of consciousness (Gregory and Shaw, 2000). However, localised lesions in the cortex, for instance in the sensory cortex, do not necessarily cause unconsciousness, but may only change specific features such as colour vision or the way visual objects and faces are interpreted (Seth et al., 2005). The central core of the brainstem is formed by the reticular formation, a large network of neural tissue located in the central region of the brain stem. The reticular formation receives sensory information from the cortex and several subcortical regions and its axons project to the cerebral cortex, thalamus and spinal cord. The reticular formation plays not only a role in sleep and arousal, but also in attention, muscle tone, movement and various vital reflexes (Carlson, 2007). When the reticular formation fails, the cerebral cortex will be switched off or cannot be switched on. When the cortex is (functionally) damaged, neuronal integration of signals from the central nervous system necessary for conscious perception and subjective experience cannot occur. The disruption of normal electrical brain activity is considered to be incompatible with consciousness (Savenije et al., 2002; Lambooij, 2004; Adams and Sheridan, 2008). To maintain consciousness, a constant supply of $\mathrm{O}_{2}$ and energy to the brain and continuous removal of metabolic waste, such as $\mathrm{CO}_{2}$, is needed. If one of the mechanisms fails, for instance due to stunning, an animal will become unconscious (Adams and Sheridan, 2008). 


\section{TIME TO AND DURATION OF UNCONSCIOUSNESS}

In a large-scale study by von Wenzlawowicz et al. (2012), stunning effectiveness was assessed in over 37000 pigs and cattle, stunned by different methods. The mean percentages for animals showing signs compatible with insufficient stunning ranged from 3 to $14 \%$, depending on the stunning method and with a high variability between slaughter plants. Gregory (2008b) found that $8 \%$ of electrically stunned cattle $(n=67)$ were not deeply stunned and showed signs of consciousness at 20 and $90 \mathrm{~s}$ post stunning. If stunning is reversible, the chance for recovery should be minimised and the stun-tostick interval should be kept to a minimum to prevent recovery during exsanguination. With electrical stunning in pigs, an interval under $15 \mathrm{~s}$ was recommended, where after exposure to gas a stun-to-stick interval of 25 to $45 \mathrm{~s}$ was advised, depending on the gas mixture and concentration used (Anil, 1991; Raj, 1999). Recommendations on the duration of stun-to-stick interval depend on different factors including the amount of current or concentration of gas used and the exposure time. When the stun is found not to be effective, the animal should be re-stunned as soon as possible. Animals that are conscious at time of the neck cut lose consciousness as a consequence of the severe decrease in cerebral blood flow leading to a rapid onset of disorganised brain function and thus unconsciousness (Mellor et al., 2009). Sheep and poultry lose spontaneous brain activity after on average 14 and $23 \mathrm{~s}$ when both carotid arteries are severed (Gregory and Wotton, 1984 and 1986). In cattle, however, consciousness after the neck cut is prolonged, as the vertebral arteries, which are not severed by the neck cut, supply blood to the circle of Willis and play a direct role in the blood supply to the brain (Baldwin and Bell, 1963a,b). Cattle lose spontaneous brain activity $75 \pm 48$ s post neck cut (range 19 to 113 s), but Newhook and Blackmore (1982b) suggested possible intermittent sensibility for up to 123 to $323 \mathrm{~s}$ after slaughter in cattle (Daly et al., 1988). The time to loss of consciousness in non-stunned animals, emphasises the need to verify unconsciousness after stunning and take sufficient time for full bleed out before the start of carcass processing, especially in cattle.

\section{ASSESSING UNCONSCIOUSNESS}

Unconsciousness, caused by temporary or permanent disruption to the brain, is generally assessed by the observation of behavioural indicators, which are internally coordinated responses to internal or external stimuli (Levitis et al., 2009). They include reflexes originating from the brain stem (e.g. eye reflexes) or spinal cord (e.g. pedal reflex) and behavioural indicators such as loss of posture, vocalisation and rhythmic breathing. In an experimental set-up, the assessment of brain activity as presented in an EEG, derivatives of the EEG, and evoked potentials can be used to assess unconsciousness. 


\section{Reflexes}

Reflexes are automatic, stereotyped movements that are produced as the direct result of a stimulus and are mediated by the central nervous system (Carlson, 2007). The presence of central reflexes are indicators of consciousness that are linked to functioning of the brain stem or spinal cord. Brain stem reflexes are regulated by 12 pairs of cranial nerves that enter and exit the brain and are not under cortical control. Two cranial nerves (I and II) enter from the forebrain and the other nerves (III to XII) enter and exit from the brain stem (Carlson, 2007; Rubin and Safdieh, 2007). Brain stem reflexes that are used to assess unconsciousness after stunning in livestock are corneal- or blinking, palpebral-, pupillary light- and threat reflex. The corneal reflex causes involuntary blinking of the eyelids in response to stimulation of the cornea and is in general the last reflex to be lost in anaesthetised animals (Dugdale, 2010). The palpebral reflex also results in blinking as a response to touching the medial canthus of the eye and disappears earlier than the corneal reflex in anaesthetised animals. Both the corneal and palpebral reflex require a functional afferent cranial nerve V (trigeminal) and efferent cranial nerve VII (facial) and the relevant eye muscles to function adequately (Adams and Sheridan, 2008). The pupillary light reflex is tested by letting light fall on the eye and observing whether the pupil adapts to it. The reflex is controlled by cranial nerves II (optic) and III (oculomotor) and is not considered a reliable reflex during exsanguination, as exsanguination interferes with the blood supply to the retina (Blackman et al., 1986). When testing the threat reflex, an object (finger or pencil) suddenly approaches the eye and a conscious animal will close its eye or withdraw the head. This reflex requires a functional efferent cranial nerve VII (facial) and integration of the motor cortex, but is not often applied, as it requires the eye to be open. Focused eye movement, not a reflex, is considered a definite sign of consciousness, as it needs cortical activity for perception and controlled motor activity from the eyeball muscles (Grillner et al., 2008; Vogel et al., 2011). It is pointed out that positive eye reflexes alone do not necessarily indicate consciousness, as positive brain stem reflexes might occur on the basis of residual brain stem activity and do not distinguish clearly between consciousness and unconsciousness (Anil, 1991). This especially holds true for animals that are electrically stunned which was documented as early as 80 years ago (Roos and Koopmans, 1936; Blackmore and Delany, 1988; von Holleben et al., 2010). In both sheep and calves, brain stem reflexes were present long after electrical stunning, even though the EEG was suppressed or iso-electric (Anil, 1991; Anil and McKinstry, 1991). On the other hand, eye reflexes may be inhibited after electrical stunning, whereas the cerebral cortex still functions and the animal may be conscious (Blackmore and Delany, 1988).

There is no literature available on the frequency of such incidences and its risk for animal welfare is there for difficult to estimate. After effective captive bolt stunning, however, no eye reflexes should be present, because of the brain trauma produced (Finnie, 1995; 
Gregory and Shaw, 2000). Thus, cranial nerve reflexes can be good indicators for impaired midbrain or brain stem activity, but only work reliably in one way: when absent, it is very likely that the animal is unconscious, but when they are present, the animal is not necessarily conscious. Spinal reflexes include stretch and flexor reflexes. The stretch reflex, a monosynaptic reflex, is the most basic reflex and plays an important role in control of posture. It does not involve the brain and is therefore not used to assess unconsciousness (Carlson, 2007). The flexor reflex, a polysynaptic reflex, involves activation of nociceptors and is used to assess unconsciousness (Anil, 1991; Erasmus et al., 2010a). An example of a flexor reflex is the pain withdrawal reflex which is elicited by applying a painful stimulus to the animal, such as a nose- or ear prick. In a survey on expert opinion, the pain withdrawal reflex was ranked high, and thus valued highly, as an indicator to assess unconsciousness after all types of stunning (Gerritzen and Hindle, 2009). The pedal reflex is elicited by, for instance, pinching the skin between the toes of an animal. This reflex is often used for assessment of depth of anaesthesia in laboratory animals, such as rodents and rabbits, but is only occasionally applied in livestock after stunning, as all spinal reflexes are difficult to assess when animals exhibit convulsions or body movements (Tidswell et al., 1987). This especially holds true for animals that are physically stunned, for example captive bolt stunning, when there is lack of inhibition from the brain and spinal reflexes may occur more vigorously (Blackmore and Delany, 1988). Again, electrically stunned animals may exhibit this reflex long after losing consciousness and the reflex may occur more vigorously when the animal is handled (Blackmore and Newhook, 1982). The righting reflex refers to any reflex that tends to bring the body into its normal upright position. It is often assessed when animals are removed from the stunning box or are hung to the bleeding rail and is also referred to the head righting reflex. This reflex is also difficult to assess when animals exhibit convulsions or involuntary body movements (Blackmore and Newhook, 1982; Anil, 1991). Table 1 shows an overview of the different brain stem- and spinal reflexes used to asses unconsciousness after stunning. 
TABLE 1

Reflexes used to assess unconsciousness in livestock after stunning

\begin{tabular}{lll}
\hline \multirow{2}{*}{ Reflex } & Definition & ${ }^{1}$ Present in animals that are \\
\cline { 3 - 3 } Brain stem reflexes & Reflexes that originate from the brain stem & Conscious $\quad$ Unconscious \\
\hline Cornea reflex & $\begin{array}{l}\text { Involuntary blinking in response to stimulation } \\
\text { of the cornea }\end{array}$ & $+(-)$ \\
\hline
\end{tabular}

\begin{tabular}{|lll}
\hline Palpebral reflex & $\begin{array}{l}\text { Involuntary blinking in response to touching the } \\
\text { medial canthus of the eye }\end{array}$ & $+(-)$ \\
\hline Pupillary light reflex & $\begin{array}{l}\text { Narrowing of the pupil in response to light that } \\
\text { falls on the retina }\end{array}$
\end{tabular}

$\begin{array}{ll}\text { Threat reflex } & \begin{array}{l}\text { Involuntary blinking or withdrawal of the head in } \\ \text { response to bringing a finger or hand with speed } \\ \text { towards the eye of an animal }\end{array} \\ \text { Spinal reflexes } & \text { Reflexes that originate from the spinal cord }\end{array}$

\begin{tabular}{|c|c|c|c|}
\hline Pain withdrawal reflex & $\begin{array}{l}\text { Withdrawal of the body part that has had a } \\
\text { painful stimulus applied to }\end{array}$ & $+(-)$ & $-(+)$ \\
\hline Pedal reflex & $\begin{array}{l}\text { Withdrawal of the foot in response to pinching } \\
\text { (the skin between) the toes of an animal }\end{array}$ & $+(-)$ & $-(+)$ \\
\hline Righting reflex & $\begin{array}{l}\text { Bringing the body into its normal position when } \\
\text { taken out of its normal upright position }\end{array}$ & $+(-)$ & $-(+)$ \\
\hline
\end{tabular}

${ }^{1}$ Presence and absence of reflexes are presented as follows: $+=$ Present, $-=$ Absent, $(+)=$ May be present, $(-)$ = May be absent. 
TABLE 1

Continued

\section{Based on}

Remarks

Functional cranial nerves originating from the brain stem

- Reflex may be present in animals that are unconscious, depending on the method of stunning (Gerritzen and Hindle, 2009).

- Absence of these reflexes though are considered valuable indicators for assessing unconsciousness (von Holleben et al., 2010).

- Cannot be tested when seizures occur (Blackmore and Delany, 1988).

Functional cranial nerves V and VII and eye muscles

- One of the most commonly used reflexes after stunning.

- In general the last reflex to be lost in anaesthetised animals (Dugdale, 2010).

- May be present after electrical stunning, but never after effective captive bolt stunning (Roos and Koopmans, 1936; Gregory and Shaw, 2000).

Functional cranial nerves II and III and eye muscles

Functional cranial nerves V and VII and eye muscles

- Disappears earlier than the cornea reflex in anaesthetised animals (Dugdale, 2010).

- Considered of little value during exsanguination, as the blood supply to the retina is restricted during this period (Blackman et al., 1986).

- Pupillary dilatation is considered a sign of total brain dysfunction (Blackman et al., 1986).

- May be absent in paralysed, though conscious animals (Blackmore and Delany, 1988).

Functional cranial nerve VII, eye muscles and - Cannot be tested when the eyes are closed. integration with motor cortex

Require a functional spinal cord, but do not necessarily require cerebral coordination
- May occur more vigorously when there is lack of inhibition from the brain (e.g. captive bolt stunning) (Blackmore and Delany, 1988).

- In a survey on expert opinion, the pain withdrawal reflex was ranked high, and thus valued highly, as an indicator to assess unconsciousness after all types of stunning (Gerritzen and Hindle, 2009).

- Difficult to assess when convulsions occur.

- Not easy to perform in all species. Mainly used in poultry.

- Difficult to assess when convulsions occur (Blackmore and Newhook, 1982; Anil, 1991). 


\section{Behavioural indicators}

Loss of posture, the inability of the animal to remain in an initial standing or sitting position, is considered a valuable indicator as it is often the first sign to be lost after successful stunning and indicates that the cerebral cortex is no longer able to control posture (Raj et al., 1992; Raj and Gregory, 1996; Llonch et al., 2013). Both mechanical and electrical stunning should lead to immediate collapse (AVMA, 2013). Nystagmus, involuntary rapid horizontal eye flickering, is caused by damage to the vestibular, labyrinthine or central nervous system and was more present in cattle that had a shallow depth of concussion following captive bolt. It was observed in only $3 \%$ of 1608 cattle, but was associated with a greater chance of rhythmic breathing. Its presence could add strength to the conclusion that the depth of concussion has been shallow (Gregory et al., 2007). In a study by Bourquet et al. (2011), nystagmus was observed in one out of 95 captive bolt shot cattle. This animal was reshot, and this supported the study by Gregory et al. (2007), which indicated that when nystagmus was observed, there was a one in three chance that the quality of the stun was insufficient. Nystagmus may occur as a result of electrical stunning (Grandin, 2002), but in $\mathrm{CO}_{2}$ stunned pigs, nystagmus was not observed once (Atkinson et al., 2012). It is stated that under no circumstances should a stunned animal vocalise, as vocalisation after stunning indicates consciousness and probably distress and pain (Grandin and Smith, 2004; Gouveia et al., 2009). A large network of brain regions is involved in the production of vocalisations, including the frontal lobe and primary motor cortex and vocalisations are considered a conscious response (Carlson, 2007). The involuntary passage of air along the vocal cords, however, may cause sounds that can be mistaken for vocalisations. Absence of vocalisations on the other hand, is certainly no guarantee for absence of pain or distress, as the occurrence of vocalisations also depends on the species. A sheep often does not vocalise when injured, where a pig will scream loudly (Broom, 2001; EFSA, 2004). Grandin (2002) believes an animal to be unconscious when it shows a limp head and protruding tongue. The tongue is controlled by nerve XII (hypoglossal) and when relaxed this may indicate loss of cranial nerve function. A study by Gregory et al. (2007) showed that a protruding tongue was not associated with depth of concussion after captive bolt stunning, but was proposed as indicator following exsanguination, when $40 \%$ of the cattle had a protruding tongue while hanging on the bleeding rail. Similarly, relaxation of the jaw may be taken into account, but can be observed in unconscious animals (Gregory et al., 2009a). Both jaw relaxation and tongue protruding are not used as single indicators to assess unconsciousness, but can support other indicators of unconsciousness (Grandin, 2002; von Holleben et al., 2010). Beside the important role regarding consciousness, the brain stem also houses the regulatory centres for respiratory and circulatory systems. Rhythmic breathing movements after stunning indicate that the corticospinal, ventral and lateral columns of the spinal cord are still intact and may thus indicate consciousness (Mitchell 
and Berger, 1975). The presence of rhythmic breathing after stunning is generally accepted to indicate that an animal may not be fully unconscious and is thought to be one of the first signs of recovery after $\mathrm{CO}_{2}$ and electrical stunning (Gerritzen and Hindle, 2009; Anastasov and Wotton, 2012). In captive bolt stunned cattle, rhythmic breathing immediately disappears after an effective shot because of axonal injuries to the brainstem (Finnie et al., 2000). The occurrence of convulsions, observed as uncontrolled movements of the body, indicates effective stunning in electrical or mechanical stunned animals, but also occur in unconscious animals that are gas stunned (Adams and Sheridan, 2008; Marzin et al., 2008; von Holleben et al., 2010). These convulsions are thought to be incompatible with consciousness due to the absence of higher motor control (Lambooij, 2004). They can, however, sometimes be mistaken for rhythmic breathing, as they can occur as almost rhythmic body movements (Wotton and Sparrey, 2002). Gagging refers to low-frequency inhalations with the neck positioned towards the front legs and occasional emission of sounds similar to snoring and is considered an indicator of deep unconsciousness (Rodríguez et al., 2008). Gasping is seen when an animal takes deep breaths through an open mouth and is considered an indicator of onset of breathlessness during $\mathrm{CO}_{2}$ stunning which continues long after loss of consciousness even when brain activity is no longer recorded, but may also occur after electrical stunning (Blackmore and Petersen, 1981; Newhook and Blackmore, 1982b; Grandin, 2013). Interpretation of all individual indicators mentioned above can be doubtful unless supported by other information (Blackmore, 1984; Gerritzen and Hindle, 2009; Anastasov and Wotton, 2012). Table 2 shows an overview of the different behavioural indicators used to asses unconsciousness after stunning. 
TABLE 2

Behavioural indicators used to assess unconsciousness in livestock after stunning

\begin{tabular}{|c|c|c|c|}
\hline \multirow[b]{2}{*}{ Indicator } & \multirow[b]{2}{*}{ Definition } & \multicolumn{2}{|c|}{${ }^{1}$ Present in animals that are } \\
\hline & & Conscious & Unconscious \\
\hline Loss of posture & $\begin{array}{l}\text { The inability of the animal to remain in an initial } \\
\text { standing or sitting position }\end{array}$ & $-(+)$ & $+(-)$ \\
\hline Nystagmus & Involuntary rapid horizontal eye flickering & $-(+)$ & $-(+)$ \\
\hline
\end{tabular}

$\begin{aligned} & \text { Vocalisations } \\ & \begin{array}{l}\text { Voluntary sounds made by the vibration of vocal } \\ \text { folds modified by the resonance of the vocal tract }\end{array} \quad+(-)\end{aligned}$
Focused eye movement Accommodation of the eye

\begin{tabular}{|c|c|c|c|}
\hline Protuding tongue & Tongue hanging from the mouth & - & $+(-)$ \\
\hline Relaxed jaw & No tension on the jaw & - & $+(-)$ \\
\hline
\end{tabular}

Limp head / no neck tension

Rhythmic breathing
Breathing consisting of rhythmic in- and exhalation
$(-)$

$(+)$

\begin{tabular}{|l|l|}
\hline Convulsions & $\begin{array}{l}\text { Uncontrolled involuntary contraction of muscles. } \\
\text { Clonic (uncontrolled jerking) and tonic (rigid) } \\
\text { activity }\end{array}$ \\
\hline Gagging & $\begin{array}{l}\text { Low-frequency inhalations with the neck towards } \\
\text { the front legs and occasional emitting of sounds } \\
\text { similar to snoring }\end{array}$ \\
\hline Deep breaths taken non-rhythmically through an \\
open mouth
\end{tabular}

${ }^{1}$ Presence and absence of reflexes are presented as follows: $+=$ Present, - = Absent, $(+)=$ May be present, $(-)=$ May be absent. 
TABLE 2

Continued

Based on

Absent when cerebral cortex can no longer control posture

Damage to the vestibular, labyrinthine or central nervous system

\section{Remarks}

- Both mechanical and electrical stunning should lead to immediate collapse (AVMA, 2013).

- More pronounced in gas stunning and starts before loss of consciousness (Raj et al., 1992; Raj and Gregory, 1996; Llonch et al., 2013).

- Should be absent, but it presence after captive bolt stunning

- could add strength to the conclusion that the depth of concussion has been shallow (Gregory et al., 2007).

- May occur as a result of electrical stunning (Grandin, 2002).

- Not observed after CO2 stunning in pigs (Atkinson et al., 2012).

A large network of brain regions that is involved in the production of vocalisations
- Can be observed while assessing other indicators and should not be present after stunning (Grandin and Smith, 2004; Gouveia et al., 2009).

- Occurrence of vocalisations is very dependent of the species. (Broom, 2001; EFSA, 2004)

Functional brain stem and cortex

- Not used as an indicator itself, though may be observed when assessing other indicators.

- Considered a definite sign of consciousness (Vogel et al., 2011).

Absence of functional cranial nerve XII and loss of control of tongue muscles

- Little reported in literature. Based on expert opinion mainly and may be gender dependent in captive bolt stunned in cattle (Grandin, 2002; EFSA, 2004; Gregory et al., 2007)

Absence of functional cranial nerve $\mathrm{V}$ and control of jaw muscles

- Little reported in literature. Based on expert opinion mainly and may depend on stunning method (Grandin, 2002; EFSA, 2004; Gregory et al., 2009).

Absence of functional cranial nerve XI and control of neck muscles

Intact corticospinal, ventral and lateral columns of the spinal cord

- May be masked when neck muscles are severed.

- Considered the first sign of potential return of consciousness following stunning (Gerritzen and Hindle, 2009; Anastasov and Wotton, 2012)

- Difficult to assess when convulsions occur.

Absence of higher centre motor control

- A lot of variation is seen between animals (Anil, 1991).

- Not thought to be compatible with consciousness (Lambooij, 2004).

- Can be misinterpreted as rhythmic breathing (Wotton and Sparrey, 2002)

Functional cranial nerves IX and $\mathrm{X}$ and control of pharynx muscles

- Considered an indicator of deep state of unconsciousness (Rodríguez, 2008)

Suppression of neuronal activities aimed at respiration in the pons and the occurrence of certain mechanisms in the medulla
- Is a first indicator of onset of breathlessness and may persist even when no brain activity is recorded anymore (Blackmore and Petersen, 1981; Newhook and Blackmore, 1982)

- May be present after electrical or CO2 stunning (Grandin, 2013) 


\section{Brain activity (EEG)}

When monitoring brain functioning, activity can be presented in an EEG which displays electrical activity derived from electrodes attached to various locations on the surface of the head. The EEG is considered the most objective method for assessing unconsciousness and reflects the sum of underlying electrical activity of populations of neurones supported by glia cells (Murrell and Johnson, 2006). There are four different types of wave patterns in the EEG that can be distinguished based on their respective frequencies and that are related to the state of consciousness: $\delta$ ( 0 to $4 \mathrm{~Hz}), \theta(4$ to $8 \mathrm{~Hz}), \alpha(8$ to $12 \mathrm{~Hz})$ and $\beta$ $(>12 \mathrm{~Hz}$ ) waves. Both $\delta$ and $\theta$ (slow wave) activity is related to sleep or reduced consciousness. $\alpha$ activity is prominent in subjects that are conscious, but mentally inactive (closing eyes and relaxation) and $\beta$ waves are associated with active movements and increased alertness (Kooi et al., 1978; Niedermeyer et al., 2011). Depending on the method of stunning, the EEG shows a characteristic pattern of change when animals lose consciousness. Generally, an increase in low frequency activity is accompanied by an increase in amplitude. When neurons depolarise at the same time or frequency, they fire in a synchronised fashion creating slow high amplitude waves as seen in unconscious states suggesting a depression of the reticular formation (Lopes da Silva, 1982). Consciousness on the other hand is characterised by high frequency ( $\alpha$ and $\beta$ ), low amplitude waves (Seth et al., 2005). When looking more specifically at EEG wave patterns, the EEG can be broken down in different time segments, better known as epochs. These epochs can be analysed for frequency $(\mathrm{Hz})$, amplitude $(\mu \mathrm{V})$ and power $\left(\mu \mathrm{V}^{2}\right)$, together representing the amount of activity in the brain. Four stages of EEG can be distinguished during the process of stunning and slaughter and are related to the level of consciousness, namely: active, transitional, unconscious and iso-electric (flat) EEG. In the first (active) stage, normal awake activity is recorded with high frequency, low amplitude waves, indicating the animal is conscious. In the second (transitional) stage, the amplitude of the EEG increases together with a decrease in frequency. When these changes become more profound, the animal is considered unconscious. When loss of consciousness progresses, the EEG turns iso-electric and brain activity is no longer recorded (Gibson et al., 2007; McKeegan et al., 2007). The exact moment when unconsciousness sets in, based on the EEG, is difficult to determine as changes are often gradual. The iso-electric EEG, however, is never compatible with consciousness. There is no consistency in the literature regarding the number of stages used in the assessment of unconsciousness. Other research may only differentiate between the stages conscious and unconscious or contrary, use additional stages besides the four mentioned above.

\section{Derivatives of the EEG}

Another way of analysing raw EEG data, next to visual appraisal of the EEG, would be to compute a Fast Fourier Transformation (FFT). The output thereof represents the 
frequency composition of the signal, or alternatively formulated, how much power is presented in the different frequency bands. The principle is similar to defining the EEG in different EEG types that consist of slow or fast waves with high or low amplitudes (Davidson, 2006). Further (automatic) calculations of the FFT can lead to EEG derivatives presenting a single value or percentage that is easier to standardise.

Derivatives of the EEG include: the total power (Ptot) which is the area underneath the frequency spectrum curve, the medium frequency (F50) which is the frequency below which $50 \%$ of the total power is located and the spectral edge frequency (F95) which is the frequency below which $95 \%$ of the power is located. These readout parameters are considered quantitative tools to describe changes in EEG activity (Murrell and Johnson, 2006). An initial increase in Ptot may represent a loss of functional cerebrocortical activity when amplitudes of EEG waves increase because of synchronised firing of neurons. But as the level of unconsciousness deepens, a decrease in all three derivatives is seen (Bager et al., 1992; Martoft et al., 2001). In electrically stunned livestock, an increase in power of all frequency bands is first observed in the first 5 to $15 \mathrm{~s}$ post-stun because of initial the epileptiform activity (Velarde et al., 2002; Beyssen et al., 2004). Automatic FFT is applied during human surgeries and on a smaller scale during animal surgeries, where the raw EEG and its FFT are used to assess anaesthetic depth. Established anaesthesia monitors are used to assess depth of anaesthesia, but they differ in the algorithm used to analyse the EEG (Bruhn et al., 2006). To the authors' knowledge, only one of such monitors, namely the Index of Consciousness or IoC, has been used in a study concerning stunning in animals. During gas stunning of pigs, the raw EEG was recorded and based on that data a dimensionless variable (IoC) was calculated (Llonch et al., 2013). This variable ranges from 100 (awake) to 0 (iso-electric) and decreases with increasing anaesthetic and sedative depth. Values between 40 and 60 are suggested to represent an adequate hypnotic effect of the subject under general anaesthesia (Grover and Bharti, 2008). In the study by Llonch et al. (2013) time to loss of posture occurred almost $20 \mathrm{~s}$ earlier then the accompanying decrease in IoC. A delay in IoC reading, compared with loss of balance, was also seen in pigs anaesthetised with propofol, but with a delay of only $7 \mathrm{~s}$ (Llonch et al., 2011). Muscular excitations that occur during $\mathrm{CO}_{2}$ stunning probably affected the IoC calculation, as movement artefacts are known to influence EEG data and calculations made in anaesthesia monitors (Teplan, 2002). This is one of the reasons offline calculation is used to more adequately compare and correlate brain activity data with behavioural indicators. Though many studies have looked at behavioural indicators or the EEG separately, only a few have studied correlations between these different readout parameters for assessing unconsciousness. In a study by Benson et al. (2012) loss of posture was correlated to the $\alpha / \delta$ ratio extracted from the EEG, in an effort to find a more objective and alternative method (as opposed to loss of posture) to assess loss of consciousness in broilers. A correlation and no difference was found between time to 
unconsciousness as observed by the two methods, supporting the use of alpha/delta ratio as method to assess unconsciousness. The study shows that such correlations can provide additional, more objective data to support the use of behavioural indicators as a measure of unconsciousness and provide details when certain behaviours may be present or absent in an animal that loses consciousness.

\section{Evoked responses}

The EEG recording is also used to assess unconsciousness by way of generating evoked responses. Evoked responses are responses in the EEG following external stimuli (visual, somatosensory or auditory), generated in specific areas of the cerebral cortex, mid brain and brainstem (Schneider and Sebel, 1997; Grover and Bharti, 2008). Evoked responses are frequently used as additional indicators to assess unconsciousness next to behavioural indicators, and have been applied in sheep, cattle, poultry and pigs. No correlations, however, have been calculated for the presence or absence of evoked potentials and presence or absence of behavioural indicators. Though, similar to EEG derivatives, evoked potentials may in this way provide additional support for the use of certain behavioural indicators. As for now, evoked responses are only used in experimental set-ups. Rapid changes in consciousness are difficult to observe with evoked potentials, as repeated stimulation and averaging of data (EEG) is needed to see these changes (Beyssen et al., 2004). Differences in time to loss of consciousness based on the loss of spontaneous EEG or evoked responses have been observed in multiple studies. In hens stunned with different gas mixtures, evoked responses were observed to disappear $\sim 15$ $\mathrm{s}$ after the EEG became suppressed, but almost $30 \mathrm{~s}$ before the occurrence of an isoelectric EEG (Raj et al., 1991 and 1992). In poultry slaughtered by nine different methods, all without prior stunning, spontaneous brain activity was lost after 23 to 233 s, where visual evoked potentials were lost after 90 to 349 s (Gregory and Wotton, 1986). The loss of somatosensory evoked potentials was also recorded before an iso-electric EEG, but after a suppressed EEG in gas-stunned turkeys (Raj and Gregory, 1993). The presence of an evoked response implies that the afferent pathways to the higher brain centres are intact, but not necessarily that the animal is aware of the stimulus (Raj et al., 1991). Visual evoked potentials have been observed in, for instance, anaesthetised animals (Gregory and Wotton, 1986; Gregory, and Wotton, 1989). Conversely, the absence of evoked potentials may not always guarantee unconsciousness (Anil et al., 2000). Gregory and Wotton (1990a) looked at the effects of multiple electrical stunning currents on spontaneous physical activity and evoked responses and found that the loss of somatosensory evoked potentials indicated a deeper level of unconscious than absence of neck tension. All these studies show that the use of different methods to assess unconsciousness may lead to different findings regarding the time to loss of consciousness. The use of absence of evoked responses or iso-electric EEG, may provide 
more conservative times to loss of consciousness compared with loss of spontaneous EEG. The indicators based on brain activity that can be used to asses unconsciousness after stunning are presented in Table 3.

\section{Difficulties in the use of EEG}

Though the EEG may be considered most objective when assessing unconsciousness, there are some disadvantages to its use. First, there is no golden standard for the way in which the division of stages of consciousness is described and this also limits the use of brain function monitors in differentiating between consciousness and unconsciousness, especially during transitional stages (Alkire et al., 2008). Second, it is difficult to compare EEG values between species and individuals, because of animal variation caused by electrode placement, skull thickness and differences between equipment. Third, the EEG can be influenced by artefacts that are animal related (eye or muscle movements) or technical related (cable movements, impedance fluctuation or $50 / 60 \mathrm{~Hz}$ interference) (Teplan, 2002). Experimental controlled situations provide a significantly better environment to limit these artefact sources than slaughter plants. These artefacts, however, limit possibilities for EEG application as an evaluation method in slaughter plants at this stage. 


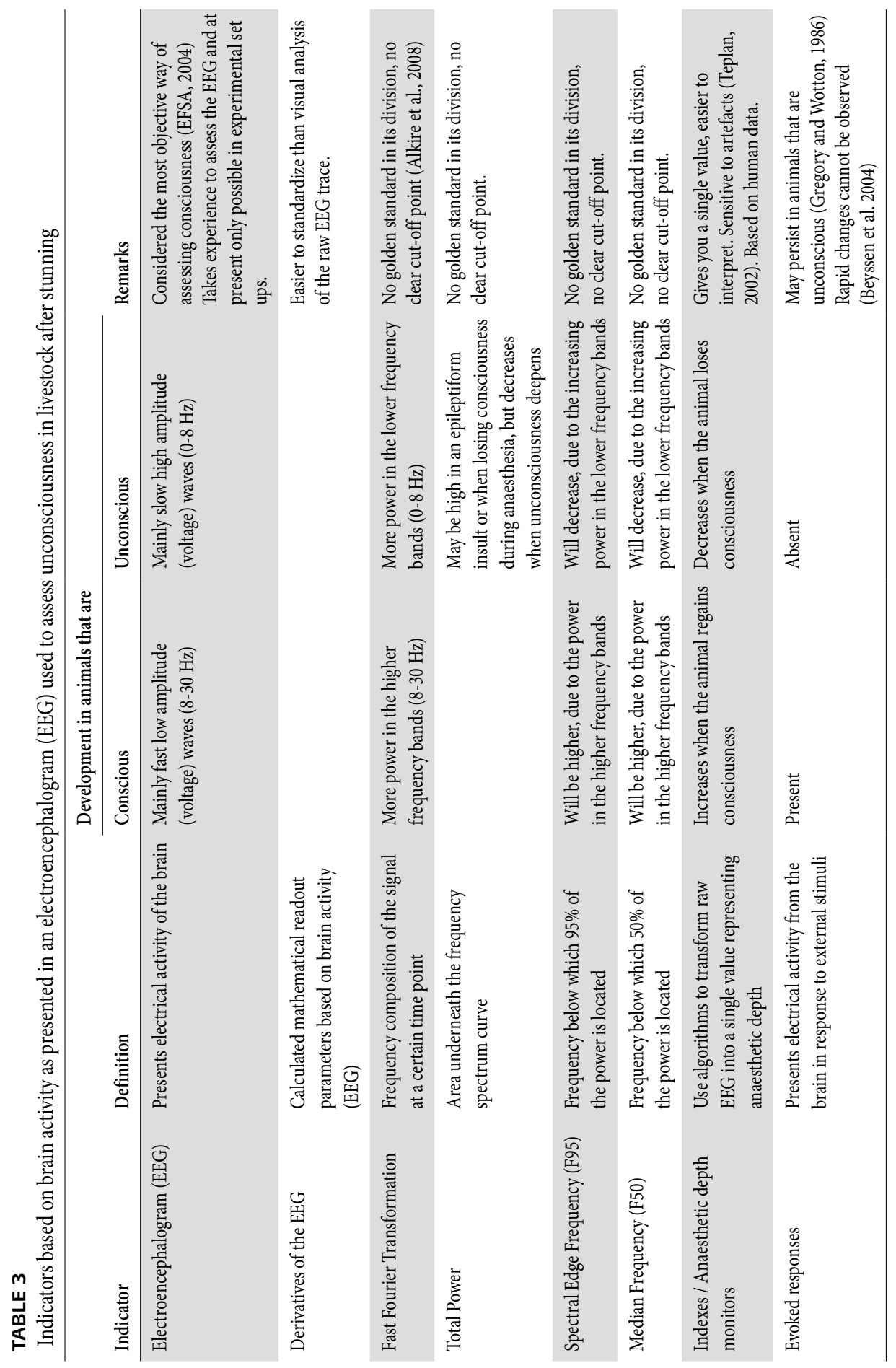




\section{CONCLUSION}

This literature review shows that there is a wide range of indicators available to assess unconsciousness in livestock after stunning. In general, pathophysiology of the consequences of the stunning method should be taken into account when assessing unconsciousness, as applicability and reliability of the different indicators vary per stunning method. When physically stunning an animal, for example, captive bolt, most important indicators are posture, righting reflex, rhythmic breathing and the corneal or palpebral reflex that should all be absent when the animal is unconscious. Spinal reflexes are difficult as a measure of unconsciousness with this type of stunning, as they may occur more vigorous. For stunning methods that do not physically destroy the brain, for example, electrical and gas stunning, most important indicators are posture, righting reflex, natural blinking response, rhythmic breathing, vocalisations and focused eye movement that should all be absent when the animal is unconscious. Brain stem reflexes such as the corneal reflex are difficult as measures of unconsciousness in electrically stunned animals, as when present they may reflect residual brain stem activity and not necessarily consciousness. It is highly recommended to use multiple indicators to definitively assess and determine unconsciousness before starting invasive dressing procedures such as scalding or skinning. The EEG is generally considered to be a most reliable indicator for assessing unconsciousness, but is (the most) difficult to apply during slaughtering due to technical-and animal related artefacts that can occur. Furthermore, the lack of a golden standard for determining (un)consciousness makes the evaluation of the EEG somewhat subjective. It is recommended to put further effort into resolving these difficulties so that the EEG can be more easily used in the assessment of unconsciousness after stunning. A substantial number of controlled studies have used the EEG to assess unconsciousness, but only one focussed on the correlation between an EEG derivative and a behavioural indicator. More research in this area should provide additional information on the absence of behavioural indicators in relation to the EEG and validate the use of certain behavioural indicators. Overall, better validated and applicable indicators are needed to reliably and reproducibly assess unconsciousness. These indicators could potentially also provide additional information on the onset of unconsciousness during the transitional period, as at present this is highly subjective, as it is often based on visual appraisal. Knowledge derived from studies using EEG in combination with other indicators in experimental set-ups could subsequently lead to improvements regarding stunning methods and subsequently animal welfare at the slaughter plant.

\section{ACKNOWLEDGEMENTS}

This study was funded by the Ministry of Economic Affairs, The Netherlands. 
Research in Veterinary Science (2015) 101: 144-153

DOI:10.1016/j.rvsc.2015.06.007

M.T.W. Verhoeven ${ }^{\star 1,2}$, M.A. Gerritzen ${ }^{1}$, M. Kluivers-Poodt ${ }^{1}$, L.J. Hellebrekers ${ }^{3}$, and B. Kemp ${ }^{2}$

${ }^{1}$ Wageningen University and Research Centre, Livestock Research, P.O. Box 338, $6700 \mathrm{AH}$

Wageningen, The Netherlands

${ }^{2}$ Adaptation Physiology Group, Department of Animal Sciences, Wageningen University, P.O. Box 338, $6700 \mathrm{AH}$ Wageningen, The Netherlands

${ }^{3}$ Faculty of Veterinary Medicine, Utrecht University, P.O. Box 80154, 3508 TD Utrecht, The Netherlands 


\section{VALIDATION OF BEHAVIOURAL INDICATORS USED TO ASSESS UNCONSCIOUSNESS IN SHEEP}

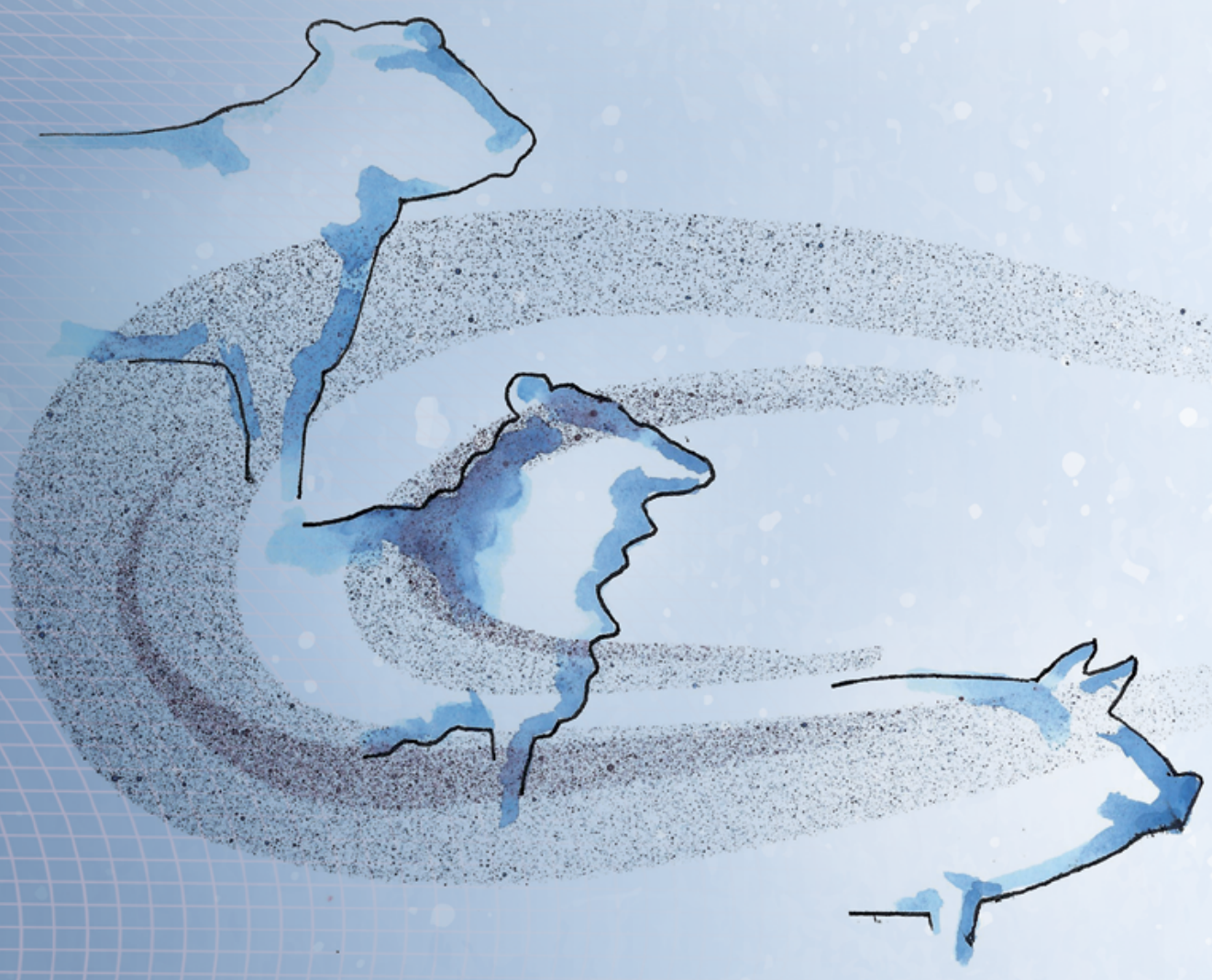




\section{ABSTRACT}

The validity of behavioural indicators to assess unconsciousness under different slaughter conditions is under (inter)national debate. The aim of this study was to validate eyelid-, withdrawal-, threat reflex and rhythmic breathing as indicators to assess unconsciousness in sheep. Sheep were monitored during repeated propofol anaesthesia $(n=12)$ and during non-stunned slaughter $(n=22)$. Changes in the EEG and behavioural indices of consciousness/unconsciousness were assessed and compared in sheep. Threat reflex and rhythmic breathing correlated with EEG activity during propofol anaesthesia whilst absence of non-rhythmic breathing or threat reflex indicated unconsciousness. None of the behavioural indicators correlated with EEG activity during non-stunned slaughter. Absence of regular breathing and eyelid reflex was observed 00:27 00:12 min and 00:59 \pm 00:17 min (mean $\pm \mathrm{SD}$ ) respectively after animals were considered unconscious, indicating that absence of regular breathing and eyelid reflex are distinctly conservative indicators of unconsciousness during non-stunned slaughter in sheep.

\section{RESEARCH HIGHLIGHTS}

The study compared EEG and behavioural indicators of consciousness/unconsciousness Behavioural indicators correlated well with EEG activity during propofol anaesthesia Behavioural indicators did not correlate well with EEG activity after non-stunned slaughter

\section{INTRODUCTION}

European legislation describes laws, rules and procedures concerning slaughter of livestock. One of these laws, namely article 5 of the Council Regulation (EC) 1099 (2009) prescribes the need to determine unconsciousness before an animal is released from restraint. An animal is considered unconscious when there is " a state of unawareness (loss of consciousness) in which there is temporary or permanent disruption of brain function. As a consequence of the disruption, the animal is unable to respond to normal stimuli, including pain" (EFSA, 2006). Unconsciousness at slaughter is generally determined by assessing absence of reflexes originating from the brain stem (e.g. eye reflexes) or spinal cord (e.g. withdrawal reflex) in combination with indicators such as loss of posture, vocalisations and rhythmic breathing (Erasmus et al., 2010a; Verhoeven et al., 2015a). There is substantial (inter)national debate on what indicators most adequately assess unconsciousness at slaughter and which merit further investigation (EFSA, 2013b). The 
use of recorded brain activity (as presented in an electroencephalogram or EEG) is considered to be the most objective method for assessing unconsciousness and is generally accepted as the current 'gold standard' (EFSA, 2012; Erasmus et al., 2010a). The EEG reflects the sum of underlying electrical activity of populations of neurones supported by glia cells (Murrell and Johnson, 2006).

The onset of unconsciousness can be determined by visual assessment of changes in patterns, amplitude and frequency of EEG activity. Generally, an increase in low frequency activity is accompanied by an increase in amplitude. When neurons depolarise at the same time or frequency, they fire in a synchronised fashion creating slow high amplitude waves as seen in unconscious states suggesting a depression of the reticular formation (Lopes da Silva, 1982). Consciousness on the other hand is characterised by high frequency, low amplitude waves (Seth et al., 2005). The onset of unconsciousness can also be determined by calculating more standardised EEG spectral variables using a Fast Fourier Transformation (FFT). The output of a FFT represents the frequency composition of the signal, or alternatively formulated, how much power is presented in the different frequency bands. Spectral variables include: total spectral power (PTot), power in the different frequency bands and spectral edge frequency (F95). PTot is calculated as the total area under the power spectrum curve, which can also be broken down into the power per frequency band. The F95 is the frequency below which $95 \%$ of the total power of the EEG is located (Murrell and Johnson, 2006). Changes in these spectral variables are known to be related to anaesthetic depth and clinical signs of unconsciousness (Martin-Cancho et al., 2003; Martín-Cancho et al., 2006; Schwender et al., 1996). In order to study absence of indicators that reflect unconsciousness, anaesthetic agents can be used to induce different stages of unconsciousness and allow for recovery, where stunning and exsanguination will lead to rapid and irrecoverable death of the animal. The use of anaesthetic agents can therefore provide a model to validate absence of the different indicators in the assessment of unconsciousness, and can provide detailed information on relations between these indicators and EEG activity. Propofol is an anaesthetic agent that inhibits neuronal firing through GABA receptor mechanisms (Schomer and Da Silva, 2012). It is used both for induction and maintenance of unconsciousness and is known for its rapid on- and offset capacity (San-Juan et al., 2010).

Slaughter without stunning involves a neck cut that severs both carotid arteries and jugular veins completely, subsequently disrupting the blood supply to the sheep's brain. In both sheep and cattle the main source of blood supply to the brain are the external carotid arteries. As a consequence of the cut, cerebral blood flow immediately decreases in sheep inducing a rapid onset of loss of brain function and unconsciousness (Baldwin and Bell, 1963a; Mellor et al., 2009). In cattle, but not in sheep, the vertebral artery also supplies blood to the brain, increasing the time to loss of consciousness (Baldwin and Bell, 1963a; Johnson et al., 2014). Time to loss of residual consciousness in sheep is on average $14 \mathrm{~s}$, 
but may last up till $5 \mathrm{~min}$ when only the jugular veins are cut (Gregory and Wotton, 1984). There is a scarcity of scientific publications reporting a correlation between unconsciousness as assessed by EEG activity and behavioural indicators of unconsciousness that could be used under slaughterhouse conditions in sheep (Rodriguez et al., 2012). The objective of this study was therefore to assess absence and presence of the following indicators in sheep: eyelid-, withdrawal-, and threat reflex and rhythmic breathing in relation to (un) consciousness as identified by visual assessment of changes in patterns, amplitude and frequency of EEG activity and changes in EEG spectral variables during propofol anaesthesia (experiment 1) and non-stunned slaughter (experiment 2).

\section{MATERIAL AND METHODS}

\section{Approval}

The experiments were approved (DEC 2013103.b) by the Ethical Committee of the Animal Sciences Group of Wageningen UR, The Netherlands.

\section{Animals and housing}

In total, 22 mixed breed ewe lambs $(36.0 \pm 3.4 \mathrm{~kg})$ from a commercial trader were used. Sheep arrived in two groups at the experimental site. The first group (group 1, $n=12$ ) arrived four days prior to start of the experiment 1 . The second group (group 2, $n=10$ ) arrived five days prior to experiment 2 and were housed together with group 1 . There were 15 days between the last day of experiment 1 and experiment 2 . All sheep were shorn prior to their arrival at the experimental facilities. The pen at the experimental site measured $5.6 \times 4.6 \mathrm{~m}(1 \times \mathrm{w})$ and was furnished with a layer of wood shavings to a depth of $\pm 5 \mathrm{~cm}$. Ambient temperature was set at $18^{\circ} \mathrm{C}$ and lights were on between $06.00 \mathrm{~h}$ and $18.00 \mathrm{~h}$. Sheep had free access to commercial feed, hay and water, except during the $16-\mathrm{h}$ prior to the experiments, when sheep only had access to water.

\section{Experimental set-up: propofol anaesthesia}

In experiment 1, all sheep were anaesthetised with propofol twice, six days apart, to minimize the number of sheep needed. Four sheep were anaesthetised per day and therefore experiment 1 comprised of two blocks of three days. When a sheep was taken out of the pen, it was transported in a cart to the experiment room. After weighing, the head and neck were shaved to enable placement of EEG electrodes and a jugular catheter. EMLA cream (EMLA', AstraZeneca, Zoetermeer), a topical anaesthetic with $2.5 \%$ lidocaine and $2.5 \%$ prilocaine, was used to provide local anaesthesia for catheter placement. The sheep was subsequently placed in a custom-made hammock (resting on the abdomen) with its legs hanging freely through four holes. After verifying the efficacy 
of the EMLA cream by pinching the sheep's skin, an 18-gauge catheter (Braun, Germany) was placed into the jugular vein, connected to a three-way tap and flushed with heparinised saline solution $(0.9 \% \mathrm{NaCl})$ and fixed to the skin. A respiratory waveform (RW) was continuously recorded by placing an inductive respiratory band $(90 \mathrm{~cm})$ around the abdomen behind the sheep's last rib (Twente Medical Systems International (TMSi), Oldenzaal, The Netherlands). Three Ag/Cl pellet electrodes, placed in a rubber sensor carrier were placed on the shaved skin of the sheep (TMSi, Oldenzaal, The Netherlands). Each electrode was wrapped in a small sponge soaked in saline solution $(0.9 \% \mathrm{NaCl})$, which served as interface with the skin. One electrode was placed over the frontal bone, on the sagittal midline on an imaginary line extending between the base of both ears. The two other electrodes were placed $2 \mathrm{~cm}$ left and right from the sagittal midline and $3 \mathrm{~cm}$ frontal from the first electrode. $3 \mathrm{M}^{\mathrm{m}}$ Vetrap ${ }^{\mathrm{Tm}}$ bandaging tape was wrapped twice around the sheep's head to secure the electrodes, leaving the ears free to move. The fourth electrode (ground electrode) consisted of an AG/AgCl electrode clipped onto a wristband soaked in saline solution, that was placed around the sheep's leg. All electrodes were connected via a $140 \mathrm{~cm}$ active shielded cable to the Porti recording system with 32 channels (TMSi, Oldenzaal, The Netherlands). The Porti system uses unipolar amplifier technology with high input impedance $(>1 \mathrm{~g} \Omega$ ) that amplifies the difference between the electrode signal and the mean of all connected electrodes (average reference). The input amplifier is dimensioned as a multichannel instrumentation amplifier. Electrode impedance was $<5 \mathrm{k} \Omega$. The EEG was displayed with a high and low frequency cut-off of 0.5 and $30 \mathrm{~Hz}$, respectively, but saved onto a computer unfiltered. Sampling rate was set at $2 \mathrm{kHz}$. Once the electrodes were placed properly and a good live signal was obtained, baseline EEG was recorded for five minutes. At $\mathrm{T}=0 \mathrm{~min}$, sheep received a bolus injection of propofol ( $8 \mathrm{mg} \mathrm{kg}^{-1}$, intravenously administered over $3 \mathrm{~min}$ ) followed by continuous infusion of propofol of $8 \mathrm{mg} \mathrm{kg}^{-1} \mathrm{~h}^{-1}$ for 28 min using a B Braun Perfusor Compact infusion pump (Type 871482/7, Braun, Melsungen, Germany). Administration of propofol stopped at $\mathrm{T}=31 \mathrm{~min}$ after which sheep regained full consciousness. Hereafter, the catheter was carefully removed and the sheep was taken to a separate part of the home pen for further recovery. At the end of the day all sheep were placed back with the flock. Six days later, the experimental procedure was repeated on every sheep.

\section{Experimental set-up: non-stunned slaughter}

In experiment 2, all sheep from group 1 and group 2 (total $n=22$ ) were slaughtered without stunning by severing both jugular veins and carotid arteries. Weighing and preparation of the sheep was similar as for propofol anaesthesia, except that catheters were not inserted in the jugular vein. Once the EEG electrodes were positioned and a good live signal was observed, a baseline EEG was recorded for $5 \mathrm{~min}$ after which the neck cut (at $\mathrm{T}=0 \mathrm{~min}$ ) was performed by a skilled halal slaughter man. 


\section{Measurements during propofol anaesthesia}

The presence of the withdrawal-, threat- and eyelid reflex was verified twice in a controlled conscious state prior to propofol anaesthesia. From $\mathrm{T}=0 \mathrm{~min}$, the responsiveness of these three indicators was tested every 2 min until sheep again showed a positive response thrice to all three stimuli and after the recorded EEG signal returned to baseline values. The withdrawal reflex was studied by pinching the sheep's tip of the ear between two fingertips and determining whether it responded by withdrawal of the ear or head. The threat reflex was assessed by checking the presence of a blinking reaction to an abrupt movement of the index finger to the eye ball without touching the eye. The eyelid reflex was assessed by a gentle touch of the eyelid, considered as present when a blinking reaction was observed and as absent when no response was observed. Stimuli to trigger responses were performed in a random order for each sheep. The EEG and RW were recorded until the EEG returned to baseline values as recorded prior to the induction of anaesthesia.

\section{Measurements during non-stunned slaughter}

The withdrawal, threat- and eyelid reflex were verified twice in a controlled conscious state prior to the neck cut. From $\mathrm{T}=0 \mathrm{~min}$, the responsiveness of these three indicators was tested every 2 seconds until a sheep showed a negative response that was repeated sequentially five times. The EEG and RW were recorded for three min after the neck cut.

\section{Data analyses}

All EEG and RW data were displayed, stored and analysed using PolyBench software (TMSi, Oldenzaal, The Netherlands). Visual analyses of EEG activity: EEG activity of each sheep was visually assessed to determine the start of the following stages: EEGbaseline, EEG-transitional $_{\text {ind }}$ (a transitional EEG during induction of anaesthesia with

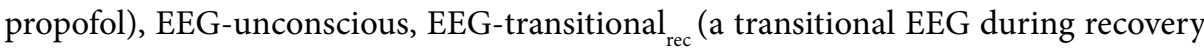
from propofol anaesthesia), EEG-recovered (an EEG similar to baseline, after propofol) and EEG-iso-electric (after neck cut). Figure 1 shows examples of representative series of four seconds of EEG registration during these different stages. EEG-baseline consisted of low-amplitude, high-frequency activity in the EEG, indicating alert sheep (Figure 1a). An EEG-transitional ind $_{\text {was }}$ determined when high-amplitude, low-frequency activity in the EEG became visually apparent, in comparison to baseline, associated with reduced consciousness (Figure 1b). When high-amplitude, low-frequency activity dominated the EEG trace, this was interpreted to indicate unconsciousness (EEG-unconscious) (Figure 1c). After the end of propofol administration, EEG-transitional rec indicated increased low amplitude, high frequency signal compared to EEG-unconscious (Figure 1d) and when recovered (EEG-recovered), the EEG would be comparable to EEG-baseline (Figure 1e). An EEG-iso-electric state reflected a flat signal ( $<10 \%$ of baseline activity) when no brain 
activity was recorded and was only seen during non-stunned slaughter (Figure 1f). Post hoc analyses of EEG spectral variables: EEG traces of each sheep were labelled with markers representing $2 \mathrm{sec}$ epochs in which there were no movement artefacts. Quantitative analysis of each epoch by FFT was used to show the frequency composition of the signal during that epoch and calculate spectral variables total power (PTot), power in the different frequency bands and F95 per epoch. This signal information was then exported into Excel and SAS for further analyses. Time points at which the PTot and power in the different frequency bands were a two-fold higher and the F95 was $4 \mathrm{~Hz}$ lower compared to baseline values were noted as start of EEG-transitional ${ }_{\text {ind }}$. Time points at which the PTot and power in the different frequency bands were a four-fold higher and the F95 was $8 \mathrm{~Hz}$ lower compared to baseline values were noted as the start of EEGunconscious. These cut-off points were based on visual observation of the raw EEG. When a clear decrease or increase was detected in EEG activity, believed to be concomitant with a change in brain activity, corresponding values were used to determine absolute values as cut-off points.

Time points at which the PTot and power in the different frequency bands returned two-fold higher and the F95 was $4 \mathrm{~Hz}$ lower compared to baseline values, after the start of EEG-unconscious, was noted as the start of EEG-transitional rec $_{\text {. Time points at which }}$ the PTot and power in the different frequency bands returned to baseline values were noted as the start of EEG-recovered. Onset of (non)rhythmic breathing was based on visual scoring of RW data and differed in its assessment during propofol anaesthesia and non-stunned slaughter. During propofol anaesthesia, breathing was considered nonrhythmic during the baseline recording and was observed when breathing changed with each breath differing somewhat in volume and frequency. Breathing was considered rhythmic when each breath became visually similar to the one before in both volume and frequency. During non-stunned slaughtered, breathing was evaluated to be regular during the baseline recording and would shift to irregular breathing following the neck cut. Irregular breathing was defined as a change in breathing from regular, normal breathing to a breathing pattern where shallow breathing, taking big gulps of air, breathing rapidly and apnoea's were alternated. Times when indicators were lost and regained were noted. 


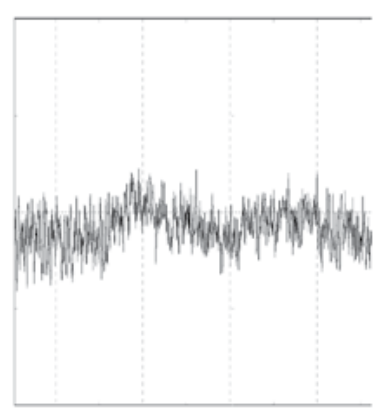

A

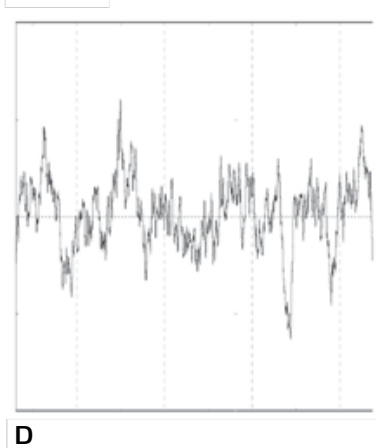

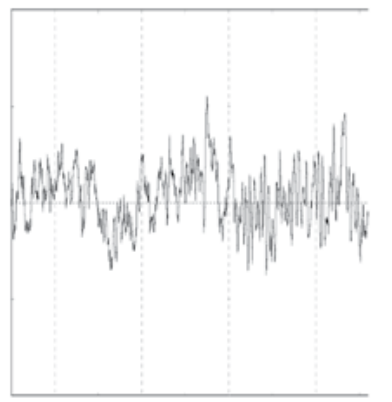

B

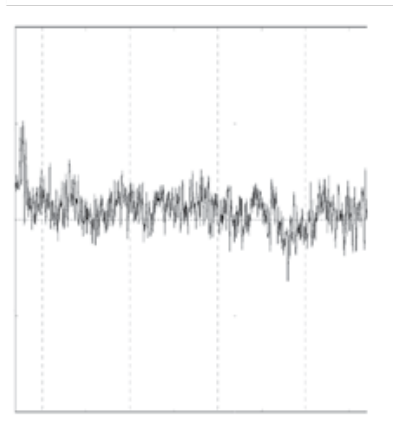

E

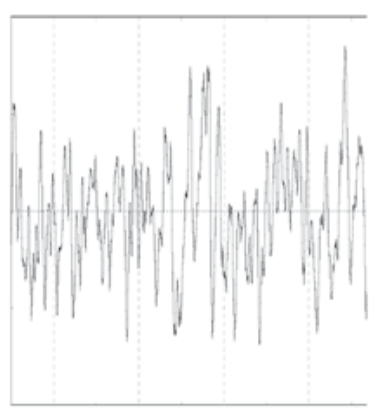

C

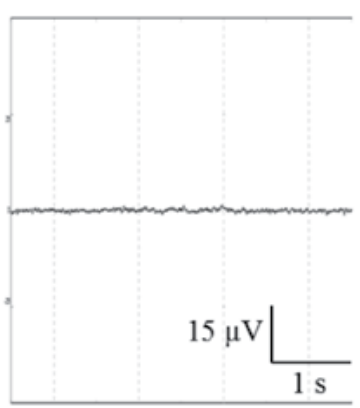

F

\section{FIGURE IA-F}

Typical example of the different stages identified with visual assessment of EEG activity prior to, during and after propofol administration and non-stunned slaughter in sheep. The six stages from left to right and top to bottom: EEG-baseline (a), EEG-transitional ind $_{\text {(b), EEG-unconscious (c), EEG-transitional }}$ (d), EEG-recovered (e), and EEG-iso-electric (f). Total X-axis represents 4 seconds, Y-axis represents amplitude of the EEG-trace $(\mu \mathrm{V})$.

\section{Statistical analyses}

Time points at which the spectral variables PTot, power in the different frequency bands and F95, first and last differed from baseline values were assessed in PROC MIXED (SAS 9.3, SAS Institute Inc.) for significant effects of time using a repeated measurement analysis with animals as the subject. Prior to the analyses, delta EEG-recovered, F95 EEG-transitional $_{\text {rec }}$, delta EEG-unconscious, PTot EEG-transitional ${ }_{\text {ind }}$ were log transformed to normalize the variables. Pearson correlation coefficients were attained between time to loss and regain of indicators and onset of different stages based on visual assessment of EEG activity using PROC CORR. T-tests were conducted to test for differences on loss of indicators and onset of EEG stages between groups 1 and 2 during experiment 2. Differences were considered to be significant at the $5 \%$ significance level. 


\section{RESULTS}

\section{Experiment 1: propofol anaesthesia}

\section{Animals}

The total number of sheep used during propofol anaesthesia was 12. One sheep in the first observation period served as pilot animal to set up and fine-tune the procedures for the experiment. During three of the 24 observations, propofol administration to the sheep was blocked temporarily, leading to a deviation in propofol doses administered compared to the other observations. As a result, a total of 20 observations from 11 sheep were used for the analyses.

\section{Propofol anaesthesia}

Anaesthesia was induced with a bolus injection of $8 \mathrm{mg} / \mathrm{kg}$ over 03:13 (range 02:03-05:15) min followed by a maintenance dose of $8 \mathrm{mg} / \mathrm{kg} / \mathrm{h}$ over the next 27:47 (range 25:45-28:57) min. Duration of anaesthesia from start of induction to discontinuation of anaesthetic drug administration was 31:00 min in all sheep. Mean time interval from induction with propofol until eye opening was 25:20 (range 20:10-33:36) min.

\section{EEG activity}

A clear EEG signal was obtained from all sheep prior to, during and after propofol anaesthesia. Onset of the different stages of consciousness based on visual assessment of EEG activity as well as spectral variables can be found in Table 1. Changes in the spectral variables PTot and the F95 before, during and after propofol anaesthesia are shown in Figure 2. Propofol anaesthesia induced an increase $(P<0.001)$ in PTot, alpha, beta, theta and delta power spectra, but decrease $(P<0.001)$ in $\mathrm{F} 95$, compared to pre-anaesthesia values. At $29 \mathrm{~min}$ after the first propofol administration none of the spectral variables remained different $(P>0.05)$ from baseline values.

\section{Loss and regain of indicators for unconsciousness}

All reflexes were present in all sheep during baseline recordings. Average times to loss and regain of the threat- and withdrawal reflex after first propofol administration ( $\mathrm{T}=0$ $\min$ ) were 01:57 \pm 00:31, 28:51 \pm 06:14 $\mathrm{min}$ and 02:48 \pm 1:14, 13:36 \pm 05:02 $\mathrm{min}$, respectively. In 8 out of 20 observations, however, the eyelid reflex was not lost at all. In those sheep that lost the eyelid reflex it was lost at on average 04:40 \pm 02:11 min and it was regained at 15:45 \pm 05:31 min. Rhythmic breathing was observed to occur from 01:26 \pm 0:21 min to 26:13 \pm 05:46 min. An overview of the average time of onset of different stages of the EEG as scored by visual assessment of EEG activity, and average time at which the indicators were lost and regained are presented in Figure $3 \mathrm{a}$. 


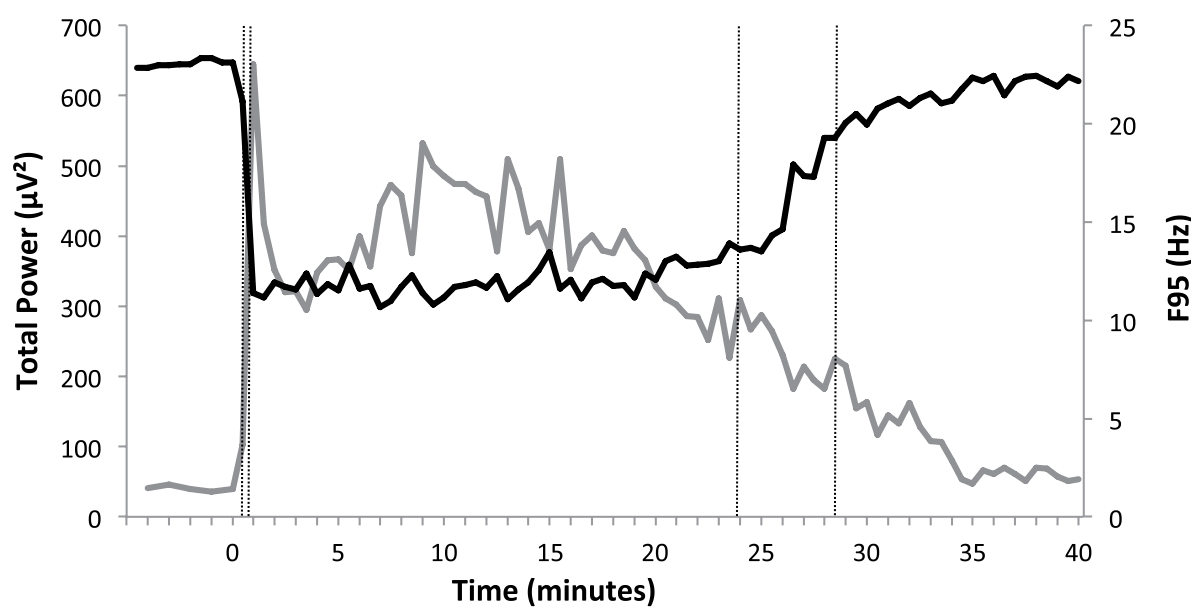

\section{FIGURE 2}

Mean total spectral power (PTot) and spectral edge frequency (F95) of the EEG averaged over 20 observations from 11 sheep prior to, during and after propofol anaesthesia. Propofol was administered from $\mathrm{T}=0$ to $\mathrm{T}=31$ min. The grey line represents total power $\left(\mu \mathrm{V}^{2}\right)$, the black line represents $\mathrm{F} 95(\mathrm{~Hz})$. Dotted vertical lines from left to right represent start of: EEG-transitionalind, EEG-unconscious, EEG-transitional ${ }_{\mathrm{rec}}$, and EEG-recovered based on visual assessment of EEG activity.

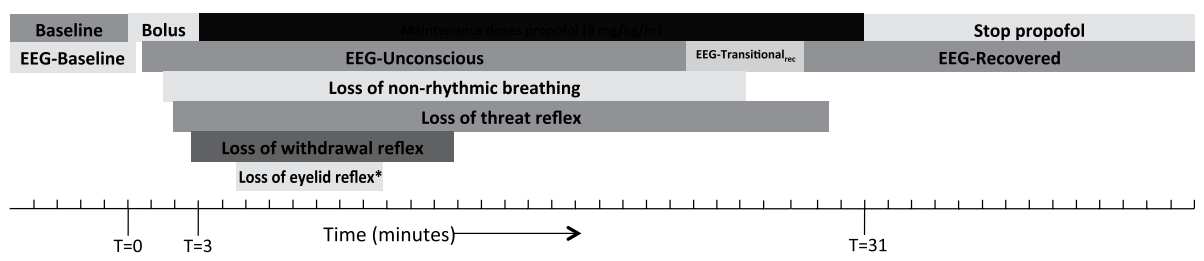

A

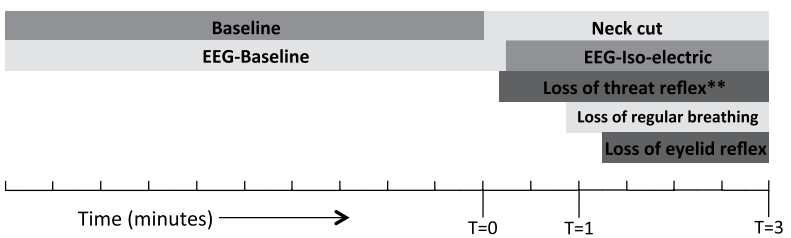

B

FIGURE 3A,B

Overview of the findings from experiment 1(Figure 3a) during which sheep (20 observations from 12 sheep) were anaesthetised with propofol from $\mathrm{T}=0$ to $\mathrm{T}=31 \mathrm{~min}$ and from experiment 2 (Figure $3 \mathrm{~b}$ ) during which sheep $(n=21)$ were slaughtered without prior stunning at $\mathrm{T}=0 \mathrm{~min}$. Upper row represents treatment to the animal. Second row represents start of different EEG stages as identified by visual assessment of EEG activity. Third to sixth row represent start and duration of loss of different behavioural indicators. ${ }^{\star}$ In 8 out of 20 observations, the eyelid reflex was not lost at all. ${ }^{\star *}$ In 14 out of 21 sheep, a threat reflex was not observed after the neck cut. 
Figure 4 represents the range of individual time points in which the different indicators were lost and regained in relation to onset of the different EEG stages ( $\mathrm{T}=0 \mathrm{~min}$ ), based on visual assessment of EEG activity (Figure 4a) and spectral variables PTot and F95 (Figure $4 b, c$ ).

All indicators were lost after an EEG-transitional ${ }_{\text {ind }}$ or EEG-unconscious was observed. These findings were similar for spectral variables PTot and F95. The threat reflex was always regained after an EEG-transitional ${ }_{\text {rec }}$ and both before $(n=6)$ and after $(n=14)$ an EEG-recovered was observed. The withdrawal reflex was regained before an EEGtransitional $_{\text {rec }}$ (with one exception where it was regained 01:35 min after an EEGtransitional rec $_{\text {) }}$ and before an EEG-recovered was observed. The eyelid reflex was regained

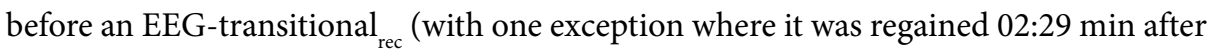
an EEG-transitional ${ }_{\text {rec }}$ ) and before an EEG-recovered was observed. Non-rhythmic breathing was regained after an EEG-transitional rec $_{\text {(with three exceptions where it was }}$ lost 14, 11 and $3 \mathrm{~s}$ before EEG-transitional ${ }_{\text {rec }}$ ) and before an EEG-recovered was observed (with two exceptions where it was regained 5 and $9 \mathrm{~s}$ after an EEG-recovered). These findings were similar for spectral variables PTot and F95.

The findings indicate that sheep that regain the threat reflex and non-rhythmic breathing after propofol anaesthesia are in a transitional, but not yet recovered stage and sheep that display a withdrawal- and eyelid reflex mostly do that before they enter a transitional or fully recovered stage. The loss and regain of indicators in relation to the power in the different frequency bands provide a similar picture as stated above and have therefore not been added to this manuscript.

\section{Correlations during propofol anaesthesia}

There were few correlations found between loss and regain of behavioural indicators and onset of the different EEG stages based on visual assessment of EEG activity during propofol anaesthesia. Correlations found included onset of rhythmic breathing with both EEG-transitional $_{\text {ind }}$ and EEG-unconscious $(\mathrm{r}=0.63, \mathrm{P}<0.001$ and $\mathrm{r}=0.74, \mathrm{P}<0.001$ respectively). Upon regaining consciousness, correlations were found between regain of the threat reflex and the EEG-transitional ${ }_{\text {rec }}$ and EEG-recovered $(r=0.81, P<0.001$ and $\mathrm{r}=0.87, \mathrm{P}<0.001$ respectively) and between loss of rhythmic breathing and EEGtransitional ${ }_{\text {rec }}$ and EEG-recovered $(\mathrm{r}=0.83, \mathrm{P}<0.001$ and $\mathrm{r}=0.87, \mathrm{P}<0.001$ respectively). 

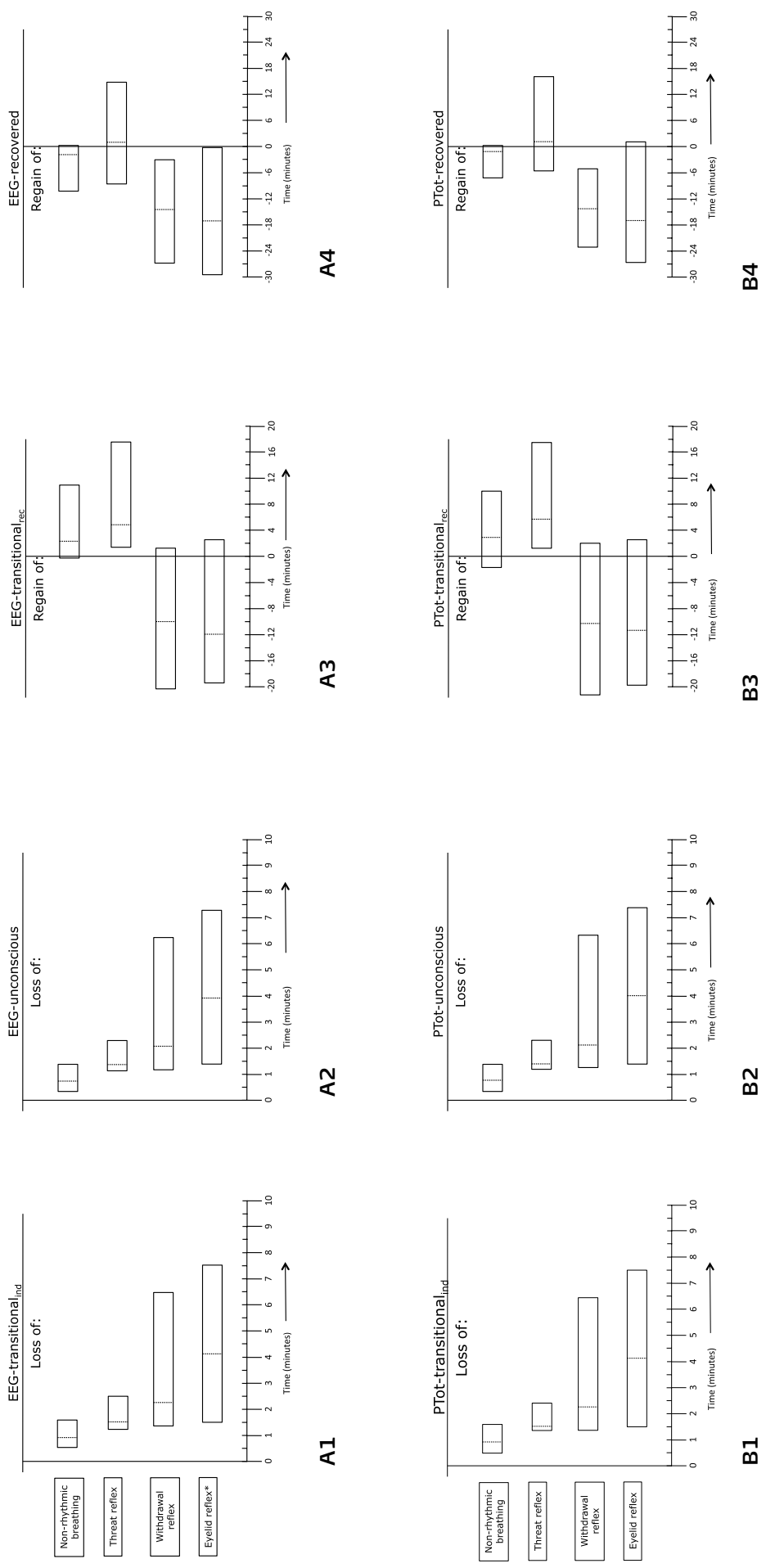

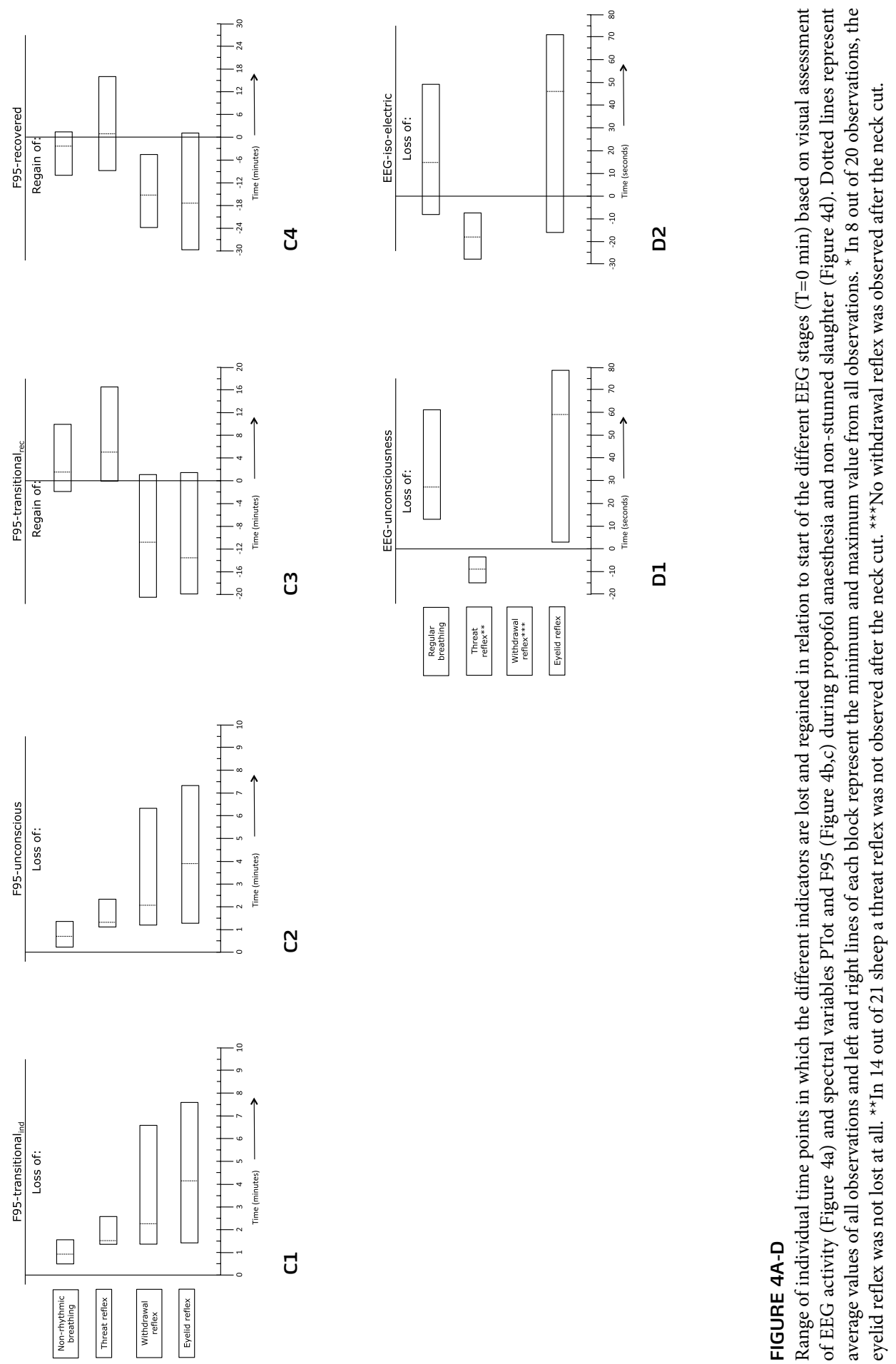

II

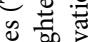

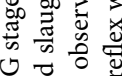

필 을

矛总起

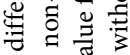

证

要

志密昰

웜 芩苛

융

인

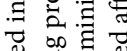

$\widetilde{\Xi}$,

㝝

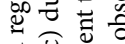

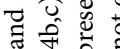

के 웡

㰻落

究会苛

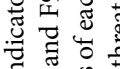

$\exists$

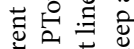

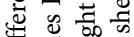

$\rightarrow \frac{1}{3}$

政

矛总

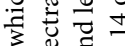

a

节完泀 영

苛总边

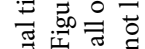

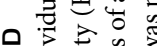

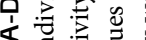

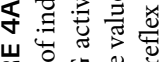

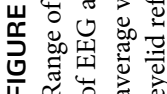




\section{Experiment 2: Non-stunned slaughter}

\section{Animals}

The total number of sheep was 22 . One sheep served as pilot animal to set up and finetune the procedures for the experiment. A total of 21 observations from 21 sheep were used in the analyses. In one observation, loss of regular breathing could not be determined due to a lost RW signal.

\section{EEG activity}

A clear EEG signal was obtained from all sheep during the $5 \mathrm{~min}$ of baseline recordings, prior to applying the neck cut ( $\mathrm{T}=0 \mathrm{~min})$. Following neck cut, the EEG was often uninterpretable for the first five to ten seconds due to interference by excessive muscle activity. After applying the neck cut, sheep were established unconscious at 00:15 \pm 00:04 min (range 0:06 - 0:24 min). No EEG-transitional was observed (Table 2). The EEG-isoelectric was observed at 00:27 \pm 00:08 $\mathrm{min}$. There were no differences in onset of the different stages between sheep from groups 1 and 2. EEG recordings could only be partially analysed, due to movement artefacts that made FFT analyses difficult. Due to the limited data per sheep, it was decided not to present the FFT analyses.

\section{Loss of indicators for unconsciousness}

All reflexes were tested and present in all sheep during baseline recordings. Seven of the 21 sheep showed a threat reflex after the neck cut, prior to loss of consciousness (until 00:07 \pm 00:01 $\mathrm{min}$ ). A withdrawal reflex was never present following neck cut, during exsanguination. An eyelid reflex, however, was observed until 01:14 \pm 00:17 min. Start of irregular breathing was observed at 00:43 \pm 00:12 $\mathrm{min}$. There were no differences in time of loss of indicators between sheep from groups 1 and 2 .

Onset of different stages as scored by visual assessment of EEG activity and time at which indicators were lost are presented in Figure $3 \mathrm{~b}$. Figure $4 \mathrm{~d}$ represents the range of individual time points in which the different indicators were lost and regained in relation to the start of the different EEG stages ( $\mathrm{T}=0 \mathrm{~min}$ ), based on visual assessment of EEG activity. The threat reflex was lost before an EEG-unconscious or EEG-recovered was observed. The eyelid reflex was lost, after an EEG-unconscious and after an EEG-iso-electric was observed (with one exception where it was lost $16 \mathrm{~s}$ before the EEG-iso-electric). Regular breathing was lost after an EEG-unconscious and after an EEG-iso-electric was observed (with two exceptions where it was lost 4 and $8 \mathrm{~s}$ before the EEG-iso-electric).

\section{Correlations during non-stunned slaughter}

There were no correlations between loss of behavioural indicators and onset of different EEG stages, based on visual assessment of EEG activity during non-stunned slaughter. 


\section{DISCUSSION}

Two experiments were designed and performed to provide insight into the validity of behavioural- and brainstem indices compared to EEG activity for the assessment of consciousness/unconsciousness in sheep. The following indicators that are used to assess unconsciousness in sheep were evaluated: eyelid-, withdrawal- and threat reflex and rhythmic breathing. Brain activity, as presented in an electroencephalogram or EEG, served as a 'gold standard' in the assessment of unconsciousness.

\section{Experiment 1: propofol anaesthesia}

Twelve sheep were anaesthetised with propofol to assess absence and presence of the indicators in relation to EEG activity. Propofol was chosen because of its limited cardiovascular effects, rapid metabolism in the liver and smooth and excitement-free induction and recovery from anaesthesia (Dugdale, 2010). The anaesthesia protocol produced was a clear transition from conscious to unconscious and induced anaesthesia for a sufficiently long period of time compared to stunning and slaughter, thus allowing the evaluation of indicators as well as EEG activity in sheep during different EEG stages. When sheep lost consciousness, the EEG changed to a characteristic pattern of highamplitude, low-frequency activity as seen in unconscious states (Baldwin and Bell, 1963b; Lopes da Silva, 1982). This increase in high-amplitude, low-frequency activity was also characterised by an increased PTot and decreased F95. The increase in PTot and decrease in F95 represent a loss of functional cerebrocortical activity when amplitudes of EEG waves increase due to synchronised firing of neurons, as described previously (Sleigh and Donovan, 1999; Upton and Ludbrook 1999). Similar results have been found during anaesthesia in a range of species and during slaughter (Johnson et al., 1994; McKeegan et al., 2013a), but there is a lack of studies that assess EEG activity and behavioural indicators simultaneously. Visual assessment of EEG activity is used in studies regarding stunning and slaughter of livestock, but this is considered somewhat subjective (Gerritzen et al., 2013; McKeegan et al., 2006). Quantitative EEG changes have been studied more recently and showed distinct changes in FFT parameters after (non) stunned slaughter in cattle. These studies showed an increase in EEG activity following non-stunned slaughter in cattle associated with pain and distress (Gibson et al., 2009b; Zulkifli et al., 2014). In non-penetrative captive-bolt stunned cattle, changes in the PTot suggest that insensibility would occur 0 to 14 s post stunning (Gibson et al., 2009c).

In experiment 1 , loss and regain of behavioural indicators in relation to the different EEG stages were assessed based on visual assessment of EEG activity and spectral variables PTot and F95. Only small differences were found between the onset of EEG stages based on visual assessment of EEG activity and those based on EEG spectral analysis, indicating that both analyses can differentiate between conscious and unconscious sheep. Monitoring 
EEG activity, however, is labour intensive and requires much experience compared to the use of behavioural indicators. Cranial nerve reflexes evaluation can help in creating an overall picture of brain (dys)function. If all are absent, this is often a strong indication of impaired midbrain or brainstem activity and unconsciousness can be established (Gregory, 1998). According to the results from the propofol experiment, the first reflex to be lost consistently and regained and with little variation among sheep when lost was the threat reflex. The induction dose of propofol induced unconsciousness with little variation in time among sheep. Despite the fact that all animals received propofol in the same dose, adjusted for weight, there was a broad variation in time of regain of consciousness. It is known that propofol is an anaesthetic to which an animal's response can show great variation between individuals, as was also observed when sheep regained consciousness in this experiment (Avsaroglu et al., 2007).

The threat reflex requires an active efferent cranial nerve VII (facial) and integration of the motor cortex to control the M. orbicularis oculi (Carlson, 2007). It was always lost when an EEG-unconscious was observed. In all observations, the reflex returned when at least an EEG-transitional ${ }_{\text {rec }}$ was observed, indicating that absence of the threat reflex always indicated unconsciousness. The withdrawal reflex is a direct motor response that does not necessarily require cerebral coordination and can occur in an unconscious animal (Carlson, 2007). This is in line with our findings where the reflex was still observed in sheep with EEG characteristics of unconsciousness during propofol anaesthesia. Benson et al. (2012) also reported inconsistent results when comb pinching birds anaesthetised with isoflurane, indicating that the brain can retain some ability to respond to external stimuli despite generalised EEG suppression. The eyelid reflex is generated by up to three different motor systems, namely facial, oculomotor and retractor bulbi. After mechanical stimulation of the periorbital skin, a blink occurs (Delgado-Garcia et al., 2003). During propofol anaesthesia, the eyelid reflex was the last reflex to be lost and the first to be regained, and it has been reported to persist occasionally during surgical stages of anaesthesia (Dugdale, 2010). The reflex may originate from some rudimentary brain stem activity, and has been observed when animals were considered unconscious, based on EEG activity (Raj, 1999). In this experiment, the reflex was not lost at all in 8 of the 20 observations. The presence of the eyelid reflex thus does not necessarily indicate consciousness. Sheep that had lost consciousness showed a rhythmic breathing pattern as a result of the effect of the anaesthetic agent on the respiratory control centre (Upton et al., 2009). During (the weaning of) propofol anaesthesia, non-rhythmic (normal) breathing re-occurred, in general after an EEG-transitional rec was observed. Only in three observations, non-rhythmic breathing re-occurred to a slight degree prior to observation of an EEG-transitional ${ }_{\text {rec }}$. The findings indicate that sheep that have lost their reflexes or show rhythmic breathing after induction with propofol are unconscious based on both visual assessment of EEG activity as well as on EEG spectral analyses. 


\section{Experiment 2: non-stunned slaughter}

Many of the studies reporting on stunning and slaughter and the effect on EEG activity have small sample sizes. A similar study to this was based on only eight observations (Rodriguez et al., 2012) and the low number of observations in these type of studies is due to the complexity of collecting EEG data at slaughter.

During the propofol study, all recordings could be taken into account during the analyses, but during non-stunned slaughter, large parts of the recordings were lost due to excessive movement artefacts. The use of EEG in general, allows for a more objective judgement of unconsciousness compared to reflexes and other indicators. But in order to apply FFT, consistent and high quality EEG data is needed. In a situation where animal- and technically-related artefacts can easily disturb the EEG signal, good quality data cannot always be acquired. The type of EEG electrodes used often depends on the type of research questions, budget and time. In poultry, EEG electrodes are often surgically implanted in or under the skull and the bird is given up to 5 days to recover prior to the experiment (Benson et al., 2012; McKeegan et al., 2013a,b). The use of cortically implanted electrodes reduces the chance of artefacts, but is more invasive to the animal (Gerritzen, personal communication). Another way of acquiring EEG data is by means of needle-electrodes that are punctured through the skin or the skull. Though, no surgery on animals is needed with this type of recording, the risk of losing data during the experiment is substantial. Multiple studies report loss of electrodes or non-readable EEG activity in 9-71\% of the animals (Velarde et al., 2002; Gerritzen et al., 2004; Lambooij et al., 2006). Video recordings linked to behavioural responses of the animal can partially explain for artefacts. Under slaughterhouse conditions it would be preferred to measure brain activity by using non-invasive electrodes on the skin surface. Development of techniques to eliminate movement artefacts that can be expected under practical conditions will be of important value. Correlations between EEG activity and reflexes or other indicators may provide a better understanding of which indicators are valid under certain circumstances, also at slaughter.

During experiment 1 , the results show that there is little variation in loss of consciousness based on visual assessment of the EEG or based on spectral variable analyses. This limited variation reflects the potential of automatically converting raw EEG signals at slaughter into FFT data. This would subsequently potentially allow for instant interpretation of such EEG data, in for instance a portable monitor. To the authors' knowledge, only one of such monitors, namely the index of consciousness (or IoC-view ${ }^{\circledast}$ ), has been applied in studies concerning stunning and slaughter of animals. With this device, raw EEG activity is recorded and processed into a dimensionless variable. This variable ranges from 100 (awake) to 0 (iso-electric) and decreases with increasing anaesthetic and sedative depth (Llonch et al., 2013). The device has been used only in an experimental set-up (Rodriguez et al., 2012; Llonch et al., 2013) regarding stunning and slaughter. For such a device to 
be used under practical circumstances, technical difficulties such as the occurrence of animal- or technical related artefacts still need to be overcome. This is one of the reasons offline calculation is now used in order to more adequately compare and correlate brain activity data with behavioural indicators.

In our experiment sheep lost consciousness, based on evaluation of the EEG, at 00:15 \pm 00:04 min post neck cut. These findings are similar to studies by Devine et al. (1986) and Gregory and Wotton (1984) who observed loss of consciousness to occur at 00:14 \pm 00:07 $\min (n=10)$ and loss of residual consciousness at 00:14 \pm 00:05 $\min (n=20)$, post neck cut, respectively. The EEG became iso-electric at 00:27 \pm 00:08 min, which was comparable to findings of Newhook and Blackmore (1982a) who observed start of an EEG-iso-electric in sheep $(n=5)$ at 00:33 $\pm 00: 13$ min post neck cut.

Only 7 out of 21 sheep had a positive threat reflex during exsanguination at an average of 00:07 \pm 00:01 min post neck cut. Since sheep were considered unconscious at 00:15 \pm 00:04 min post neck cut, absence of the threat reflex did not necessarily indicate unconsciousness. The rapid loss of the threat reflex can possibly be explained by the sudden drop in blood flow following the cut causing hypoxia of the brain cells involved (Bourguet et al., 2011). No withdrawal reflex was observed after the neck cut, though the animals were considered conscious based on EEG activity. Beside the veins and arteries, the knife also transects other blood vessels, skin, muscle, trachea, oesophagus, sensoryand motor nerves, and connective tissue. Transecting these soft tissues will cause a major amount of neural impulses to travel to the brain. This produces an intense, but brief injury discharge in the afferent nerves. The cut end of these nerves depolarises and is unable to respond to further stimuli. The massive stimulation of all sensory nerves after the neck cut can lead to a state of shock and distress (Gregory, 2008a). Therefore, animals most likely did not respond to another and milder source of pain in the current study (Johnson et al., 2012).

The occurrence of pain from the neck cut itself and possible pain and distress that may occur before onset of unconsciousness is one of the welfare concerns regarding nonstunned slaughter (Gibson et al., 2009b). Gibson et al. (2009b) quantitatively examined the noxiousness of slaughter by ventral-neck incision. The results demonstrated that ventral-neck incision caused EEG changes which were quantitatively and qualitatively similar to those observed following scoop dehorning (Gibson et al., 2007). In combination with previous analyses (Mellor and Littin, 2004), these changes demonstrated that ventralneck incision has strong potential to be perceived as a noxious stimulus and therefore to be painful in conscious animals subjected to this procedure, which was also supported by Zulkifli et al., 2014. A study by Gibson et al., 2009a support the conclusion that the EEG responses seen after a ventral-neck incision are primarily due to noxious stimulation due to the incision of ventral-neck tissues and not as a result of loss of blood flow through the brain. Opinions on this subject are divided, however, and claims have been made 
stating that with a clean incision made with an exquisitely sharp knife, significant pain and distress are minimized (e.g. Grandin, 1994; Rosen, 2004). Until now, neurophysiological methodology has not provided the absolute answer to this issue.

The eyelid reflex was lost at 01:14 \pm 00:17 min after the neck cut. Based on the EEG, sheep had at that time long lost consciousness and an EEG-iso-electric was at that time observed in 20 out of 21 sheep. As during anaesthesia, this reflex is known to persist for a relatively long time. Both experiments described in this paper support the idea that a positive eyelid reflex alone does not necessarily indicate consciousness, since positive brain stem reflexes might occur on the basis of residual brain stem activity and do not distinguish clearly between consciousness and unconsciousness (Anil, 1991). Despite that the eyelid reflex has been critically appraised by multiple experts, its absence however is still uniformly considered a reliable indicator of unconsciousness, unless the optic nerve is impaired thus prohibiting a response (Gregory and Shaw, 2000). Rhythmic breathing movements indicate that the corticospinal, ventral and lateral columns of the spinal cord are still functionally intact and this breathing pattern may thus indicate (a certain degree of) consciousness (Mitchell and Berger, 1975). Consequently, the absence of rhythmic breathing is generally accepted as a reliable indicator of unconsciousness, although the evaluation of the breathing patterns may be difficult, especially in restrained animals. During exsanguination, sheep lost regular breathing 00:43 \pm 00:12 min after the neck cut. These findings are comparable to a study of Rodriguez et al. (2012) where rhythmic breathing was lost at on average 00:44 \pm 00:04 min post neck cut in non-stunned sheep. Exsanguination will affect the central nervous system (ischaemia) in a graded process from the cortex down, where anaesthesia will affect the central nervous system globally. During exsanguination the brain stem may remain functional for some time after the cortex has failed and this explains why time to loss of regular breathing occurred 00:27 \pm 00:12 min later than time to loss of consciousness in non-stunned slaughtered sheep compared to sheep anaesthetised with propofol. Based on the EEG, all sheep had long lost consciousness after regular breathing was lost and an EEG-iso-electric was observed in 18 of 20 observations. The combined results indicate that when rhythmic breathing is absent in non-stunned sheep, it is very likely that the animal is unconscious, but when present, the animal does not necessarily need to be conscious.

\section{CONCLUSIONS}

Both experiments induced unconsciousness in sheep and linked behavioural indicators to EEG activity. The use of behavioural indicators to assess unconsciousness can more easily be applied in daily practice, but EEG recordings can be used to validate such indicators. During propofol anaesthesia, absence of non-rhythmic breathing and absence 
of the threat reflex reflected unconsciousness. After non-stunned slaughter, absence of the eyelid reflex and absence of regular breathing reflected unconsciousness, but both appeared distinctly conservative indicators of unconsciousness. In general, the (pharmacological) background of the anaesthesia or the slaughter method should be taken into account when assessing indicators for unconsciousness, since applicability and reliability of the different indicators may vary between methods used. Using both visual analyses as well as spectral analyses of the EEG signal it was possible to differentiate between different stages of unconsciousness during propofol anaesthesia with little variation between results of the visual- and spectral analyses. This limited variation supports the potential of automatically converting raw EEG signals at slaughter into FFT data and subsequent instant interpretation of such EEG data establishes unconsciousness of an animal. This study is one of the first studies to look at behavioural indicators as well as corresponding EEG activity. It helps understanding interpretation of different indicators for unconsciousness in sheep.

\section{CONFLICT OF INTEREST}

There were no conflicts of interest.

\section{ACKNOWLEDGEMENTS}

The authors would like to thank Dirk Anjema, Henny Reimert and Rob Hartman for their technical support while collecting the data. Jan Peuscher is acknowledged for both his technical assistance while collecting the data as well as his help during data analyses. The authors would also like to acknowledge the animal caretakers of the Central Veterinary Institute for taking care of the sheep. Funding for this project was provided by the Dutch Ministry of Economic Affairs. 


\section{Animal (2016)11:1-9}

DOI: $10.1017 / S 1751731116000422$

M.T.W. Verhoeven ${ }^{1,2}$, M.A. Gerritzen ${ }^{1}$, L.J. Hellebrekers ${ }^{3}$ and B. Kemp ${ }^{2}$

${ }^{1}$ Wageningen University and Research Centre, Livestock Research, P.O. Box 338, $6700 \mathrm{AH}$ Wageningen, The Netherlands

${ }^{2}$ Adaptation Physiology Group, Department of Animal Sciences, Wageningen University, P.O. Box 338, 6700 AH Wageningen, The Netherlands

${ }^{3}$ Faculty of Veterinary Medicine, Utrecht University, P.O. Box 80154, 3508 TD Utrecht, The Netherlands 


\section{VALIDATION OF INDICATORS USED TO ASSESS UNCONSCIOUSNESS IN VEAL CALVES AT SLAUGHTER}

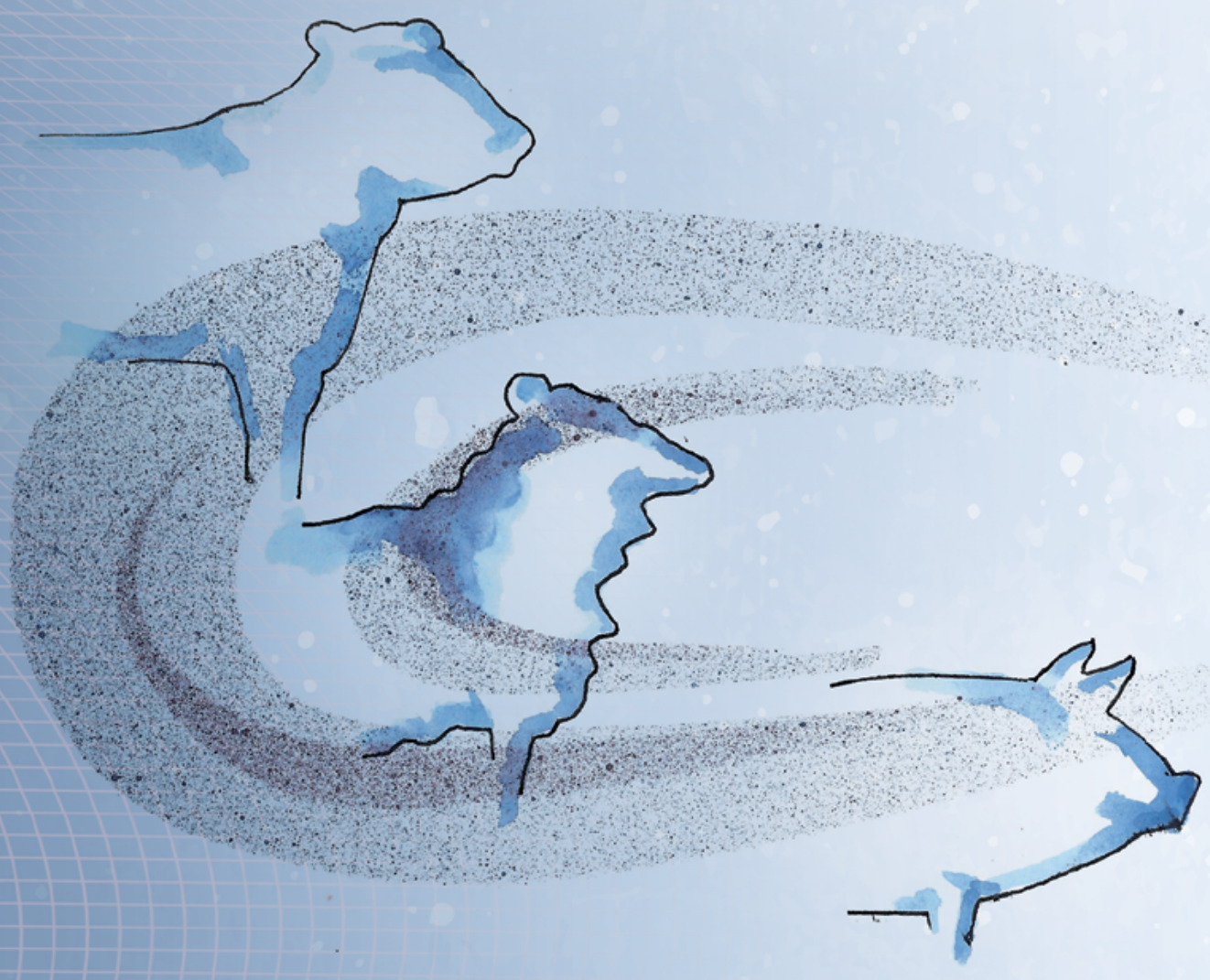




\section{ABSTRACT}

European legislation states that after stunning regular checks should be performed to guarantee animals are unconscious between the end of the stunning process and death. When animals are killed without prior stunning these checks should be performed before the animal is released from restraint. The validity of certain indicators used to assess unconsciousness under different stunning and slaughter conditions is under debate. The aim of this study was to validate the absence of threat-, withdrawal-, corneal-, and eyelid reflex as indicators to assess unconsciousness in calves subjected to different stunning and slaughter methods. Calves $(201 \pm 22 \mathrm{~kg})$ were randomly assigned to one of the following four treatments: (1) Captive bolt stunning followed by neck cut in an inverted position ( $n=25)$; (2) Non-stunned slaughter in an upright position ( $n=7)$; (3) Nonstunned slaughter in an inverted position $\left(180^{\circ}\right.$ rotation) $(n=25)$; (4) Non-stunned slaughter in an upright position followed by captive bolt stunning $40 \mathrm{~s}$ after the neck cut $(n=25)$. Each calf was equipped with non-invasive electroencephalogram (EEG) electrodes before the slaughter procedure. All reflexes were verified once before the slaughter procedure. At the beginning of the procedure $(\mathrm{T}=0 \mathrm{~s})$ calves were stunned (treatment 1) or neck cut in an upright position (treatment 2,4 ) or inverted position (treatment 3). Calves of treatment 4 were captive bolt stunned $34 \pm 8 \mathrm{~s}$ after the neck cut. Reflexes were assessed every $20 \mathrm{~s}$ from $\mathrm{T}=15 \mathrm{~s}$ for all treatments until all reflex tests resulted in a negative response three times in a row and a flat line EEG was observed. In addition, reflexes were assessed $5 \mathrm{~s}$ after captive bolt stunning in calves of treatments 1 and 4 . Visual assessment of changes in the amplitude and frequency of EEG traces was used to determine loss of consciousness. Timing of loss of consciousness was related to timing of loss of reflexes. After captive bolt stunning, absence of threat-, withdrawal,corneal,- and eyelid reflex indicated unconsciousness as determined by EEG recordings. After non-stunned slaughter, both threat- and withdrawal reflex were on average lost before calves were unconscious based on EEG recordings. The eyelid- and corneal reflex were on average lost after calves had lost consciousness based on EEG recordings and appeared to be distinctly conservative indicators of unconsciousness in non-stunned slaughtered calves since they were observed until $76 \pm 50 \mathrm{~s}$ and $85 \pm 45 \mathrm{~s}$ (mean $\pm \mathrm{SD}$ ) respectively after EEG-based loss of consciousness.

\section{IMPLICATIONS}

Monitoring unconsciousness at slaughter is mandatory by European legislation and is often determined by the absence of behavioural indicators (i.e. loss of posture), physical signs (i.e. rhythmic breathing) and reflexes. Previous research in sheep has indicated that 
the absence of certain reflexes at slaughter is not a reliable indicator of unconsciousness. The present study showed that absence of the withdrawal- and threat reflex did not indicate unconsciousness in veal calves subjected to non-stunned slaughter and are therefore not recommended for assessment of unconsciousness. Absence of the eyelidand corneal reflex, on the other hand, reflected unconsciousness well and are considered acceptable for assessment of unconsciousness during non-stunned slaughter.

\section{INTRODUCTION}

Animals are stunned before slaughter to minimize pain and distress as required by European law (Council Regulation (EC) 1099, 2009). Stunning, however, is not always compatible with specific methods of slaughter prescribed by religious rites and is legally determined not to be mandatory in animals subjected to this type of slaughter (Council Regulation (EC) 1099, 2009). Stunning after neck cutting is practiced in some European countries in preference to no stunning at all to avoid potential animal welfare problems (Farouk, 2013). Assessment of unconsciousness, is a legal requirement during the slaughter process in Europe irrespective of the method used (Council Regulation (EC) 1099, 2009).

During non-stunned slaughter, animals are restrained and bled by a transverse incision of the neck, severing the skin, muscles, trachea, oesophagus, carotid arteries, jugular veins and major nerves. The severe decrease in cerebral blood flow is intended to lead to a rapid onset of disorganized brain function and unconsciousness (Mellor et al., 2009). In comparison to sheep, consciousness after the neck cut is prolonged in cattle, because the vertebral arteries, which are not severed by the neck cut, continue to supply blood to the circle of Willis and provide some blood to the brain (Baldwin and Bell, 1963a,b). There is considerable variation between cattle in time to loss of consciousness after the neck cut. Some studies suggest a rapid loss of consciousness (4 to $7 \mathrm{~s}$ ) in cattle after the neck cut (Nangeroni and Kennett, 1963; Bager et al., 1992) whereas other studies report a spontaneous loss of brain activity $75 \pm 48 \mathrm{~s}$ after the neck cut (range 19 to $113 \mathrm{~s}$ ) and the possibility of an intermittent return to consciousness for up to 123 to $323 \mathrm{~s}$ after slaughter (Newhook and Blackmore, 1982b; Daly et al., 1988).

There is substantial debate about which indicators most adequately assess unconsciousness at slaughter and which merit further investigation (European Food Safety Authority (EFSA), 2013b). The use of recorded brain activity (as presented in an electroencephalogram or EEG) is considered to be the most objective method available for assessing unconsciousness and is generally accepted as the current 'gold standard' (Erasmus et al., 2010a; EFSA, 2013b; Verhoeven et al., 2015a). Due to the complexity of collecting EEG data at slaughter, absence of behavioural indicators (i.e. loss of posture) 
physical signs (i.e. rhythmic breathing) and reflexes (i.e. righting-, threat-, withdrawal-, corneal, and eyelid reflex) is often used to assess unconsciousness. However, there is a lack of scientific publications reporting relationships between unconsciousness ascertained by EEG recordings with those assessed using behavioural indicators, physical signs or reflexes that could be used in slaughterhouse conditions in bovines.

It has been formally agreed in The Netherlands that animals subjected to non-stunned slaughter should be unconscious within $40 \mathrm{~s}$, based on absence of at least 3 of the following 5 indicators: (1) threat reflex; (2) withdrawal reflex; (3) corneal reflex; (4) (spontaneous) eyelid reflex; (5) rhythmic breathing (Staatscourant, 2012). Different studies showed that the corneal- and eyelid reflex and rhythmic breathing were lost long after animals were considered unconscious based on EEG recordings, making them distinctly conservative indicators when assessing unconsciousness in non-stunned slaughtered sheep (Verhoeven et al., 2015b) and veal calves (Lambooij et al., 2012). On the contrary, both threat- and withdrawal reflex were often lost before sheep were unconscious according to EEG recordings during non-stunned slaughter (Verhoeven et al., 2015b).

The objective of the current study was to assess absence and presence of the following indicators: threat-, withdrawal-, corneal-, and eyelid reflex and determine their relationship to (un)consciousness as identified by EEG recordings during stunned and non-stunned slaughter in veal calves.

\section{MATERIAL AND METHODS}

\section{Approval}

This study was approved (DEC 2014045.b) by the Ethical Committee of the Animal Sciences Group of Wageningen UR, The Netherlands and in compliance with Dutch legislation.

\section{Experimental set-up}

On seven occasions, calves subjected to (non)stunned slaughter were observed at an abattoir in The Netherlands in the period from September to November 2014. Eighty-two cattle of mixed breeds (warm carcass weight $201 \pm 22 \mathrm{~kg}$ ) were randomly selected from groups in lairage and held individually in a rotation box (Nawi, Borculo, The Netherlands) while equipped with non-invasive EEG electrodes. Calves were randomly assigned to one of the following four treatments: (1) Captive bolt stunning (Cash Magnum $9000 \mathrm{~s}$ ) followed by neck cut within $30 \mathrm{~s}$ in an inverted position ( $180^{\circ}$ rotation) ( $\left.n=25\right)$; (2) Nonstunned slaughter in an upright position $(n=7)$; (3) Non-stunned slaughter in an inverted position ( $180^{\circ}$ rotation) $(n=25)$; (4) Non-stunned slaughter in an upright position followed by captive bolt stunning $40 \mathrm{~s}$ after the neck cut $(n=25)$. 
Treatment group 2 was limited to seven calves because of animal welfare concerns: that is delayed induction of unconsciousness due to insufficient bleeding. Calves were restrained with a head yoke and chin lift, but without a belly supporting plate. The neck cut was performed as a clean transverse incision of the neck, severing the skin, muscles, trachea, oesophagus, carotid arteries, jugular veins and major nerves. All stunning and slaughter procedures were performed by the same skilled halal slaughter man.

\section{Electroencephalogram recordings}

After being positioned in the restrainer, the head of the animal was shaved with an electrical trimmer (Aesculap favorita II GT104, Braun Suhl GmbH, Germany) to enable placement of EEG electrodes. Thereafter, an elastic halter was placed around the calves head. A cross-shaped piece of cotton was attached to the halter containing Velcro parts at each end of the cross. A 'shooting-hole' was cut in the middle of the cross, through which the captive bolt could be administered. Five rubber sensor carriers were placed around the 'shooting-hole' (Figure $1 \mathrm{a}$ and $\mathrm{b}$ ). Five $\mathrm{Ag} / \mathrm{Cl}$ pellet electrodes (Twente Medical Systems International (TMSi), Oldenzaal, The Netherlands) were placed in the rubber sensor carriers to make contact with the shaved skin of the calf. Each electrode was wrapped in a small sponge soaked in saline solution, which served as interface with the skin. One electrode was placed over the frontal bone, on the sagittal midline on a line extending between the base of both ears. Two electrodes were placed $2 \mathrm{~cm}$ left and right from the sagittal midline and $3 \mathrm{~cm}$ frontal to the first electrode, the other two electrodes were placed $2 \mathrm{~cm}$ left and right from the sagittal midline and $6 \mathrm{~cm}$ frontal to the first electrode. An elastic band was wrapped once around the calves' head to secure the upper three electrodes, but leaving the ears and eyes free to move.

All electrodes were connected via a $140 \mathrm{~cm}$ active shielded cable to the 32-channel Porti recording system (TMSi). Porti uses bipolar amplifier technology with high input impedance $(>1 \mathrm{G} \Omega$ ) that amplifies the potential difference between pairs of electrodes. The input amplifier is dimensioned as a multichannel instrumentation amplifier. Electrode impedance was $<5 \mathrm{k} \Omega$. The EEG was displayed with a band pass filter of 0.5 and $30 \mathrm{~Hz}$, respectively, and raw, unfiltered, data were saved directly onto a computer. Sample rate was set at $0.5 \mathrm{kHz}$. Once the electrodes had been positioned correctly and a good live signal had been established, baseline EEG activity was recorded for at least 2 min.

At the beginning of the slaughter procedure $(\mathrm{T}=0 \mathrm{~s})$ calves were stunned (treatment 1 ) or neck cut in an upright position (treatment 2,4) or inverted position (treatment 3 ). Calves of treatment 4 were captive bolt stunned $34 \pm 8 \mathrm{~s}$ after the neck cut. The EEG was recorded until a flat EEG ( $<10 \%$ baseline amplitude) was observed and all reflexes showed absence of response that was repeated sequentially three times. Hereafter, the equipment was carefully removed from the calf after which it was released from the restrainer to allow further processing of the carcass. 


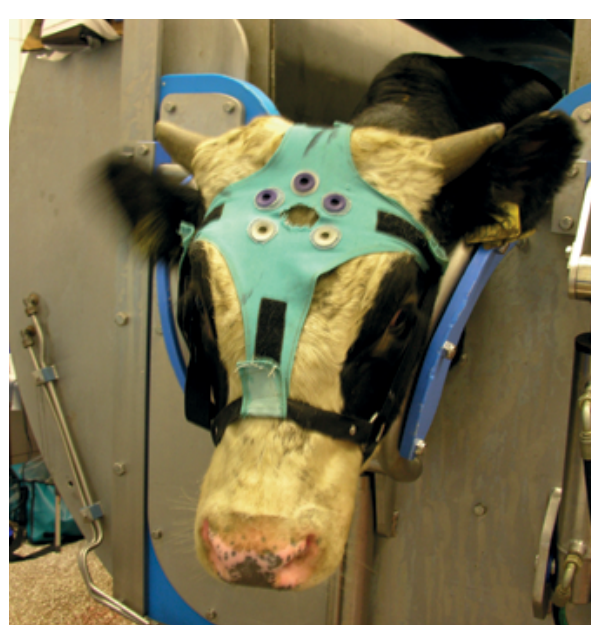

A

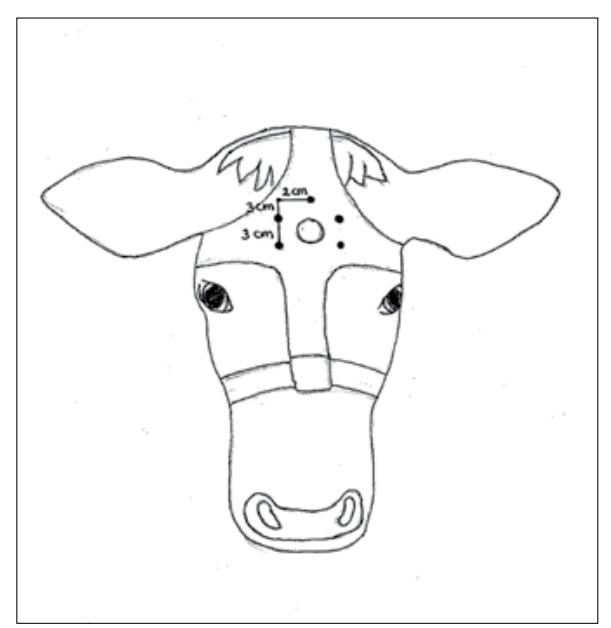

B

FIGURE $1 A, B$

Electroencephalogram (EEG) electrode placement on a veal calf.

\section{Measurements around stunning and slaughter}

The threat-, withdrawal-, corneal-, and eyelid reflex were verified once before the slaughter treatment started. The threat reflex was assessed by checking the presence of a blinking reaction to an abrupt movement of the index finger towards the eye ball without touching the eye. The withdrawal reflex was studied by pinching the calves nose between two fingertips and determining whether or not it responded with a withdrawal of the nose or head. In case of non-stunned slaughter only withdrawal of the nose could be observed after the neck cut. The corneal reflex was assessed by a gentle touch of the cornea with the index finger, and the presence/absence of a blinking reaction was recorded. The eyelid reflex was assessed by a gentle touch of the eyelid, and the presence/ absence of a blinking reaction was recorded. Reflexes were assessed every $20 \mathrm{~s}$ from $\mathrm{T}=15$ $s$ onwards for all treatments until all reflex tests showed a negative response that was repeated sequentially three times and a flat EEG was recorded. In addition, reflexes were assessed $5 \mathrm{~s}$ after captive bolt stunning in calves of treatment 1 and 4 . Reflex tests were performed in a random order per calf.

\section{Data analyses}

EEG data were displayed, stored and analysed using PolyBench software (TMSI, Oldenzaal, The Netherlands). EEG activity (amplitude and frequency) of each calf was visually assessed to determine the start of the following stages: baseline, transitional, unconscious and minimal brain activity. Figure 2 shows an example of a representative series of $5 \mathrm{~s}$ of EEG registration during these different stages. The baseline stage consisted of a low amplitude, high frequency signal, indicating alert calves (Figure 2a). 
The transitional stage is characterised by low frequency, high amplitude (firing of neurons in a synchronised fashion) becoming more apparent compared with baseline, associated with reduced consciousness (Figure 2b, McKeegan et al., 2013b). When low frequency, high amplitude dominated the EEG trace this was interpreted to indicate unconsciousness (Figure 2c, Baars et al., 2003). Minimal brain activity was reflected by a flat signal $(<10 \%$ of baseline amplitude, Figure $2 \mathrm{~d}$ ). Time to loss of a reflex was defined as the first time at which a reflex showed a negative response that was repeated sequentially three times, since loss and return of a reflex was observed in some calves.

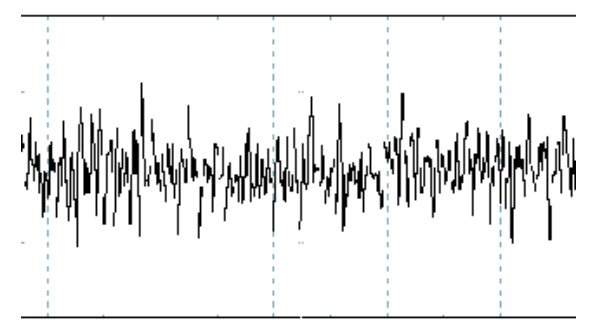

A

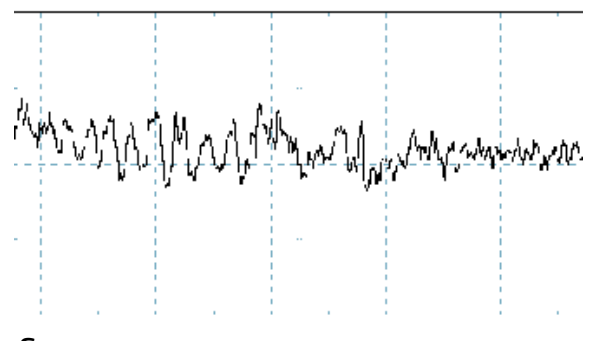

C

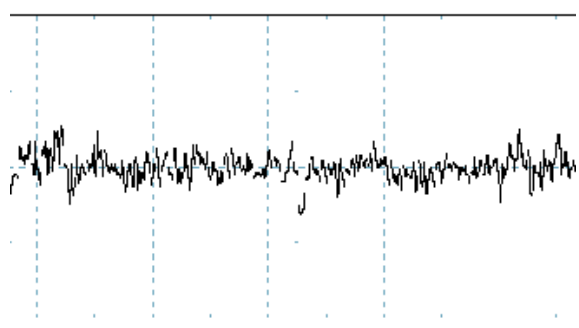

B

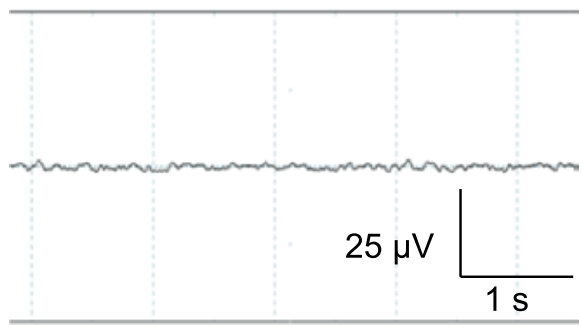

D

FIGURE 2A-D

Representative examples of the different stages identified with visual assessment of electroencephalogram (EEG) activity before and after stunned and non-stunned slaughter in calves. The four stages from left to right and top to bottom: baseline (a), transitional (b), unconscious (c), and minimal brain activity (d). Total X-axis represents 5 seconds, Y-axis represents amplitude of the EEG-trace $(\mu \mathrm{V})$.

\section{Statistical analyses}

To calculate sample size, a power analysis was performed using the Power Calculation version 2.03 programme, designed by van Wilgenburg and van Schaick Zillesen, 2003 version. Results indicated that a sample of at least 10 calves per treatment would be required to detect a difference of $20 \mathrm{~s}$ in loss of consciousness with $90 \%$ power using a $t$-test between means with $\alpha$ at 0.05 . The sample size was increased to 25 calves per treatment to allow for possible loss of EEG recordings. 
Statistical analyses were performed using SAS (version 9.3; SAS Institute, 2004). Normality of variance was checked (PROC UNIVARIATE) for all the variables examined. Before the analyses, the variables 'time to lost eyelid reflex' and 'time to lost corneal reflex' were $\log$ transformed to normalize the variance within these variables. In order to determine whether or not non-stunned slaughter in an upright or inverted position had a significant effect on time to onset of different EEG stages and time to loss of reflexes, data were analysed using a GLM procedure according to the model:

$$
\mathrm{Y}_{\mathrm{i}}=\mathrm{T}_{\mathrm{i}}+\varepsilon_{\mathrm{i}}
$$

where $\mathrm{T}_{\mathrm{i}}$ is the treatment ( 2 and 3 ), and $\varepsilon_{\mathrm{ij}}$ the residual error term.

Stunned slaughter (treatments 1 and 4) was not taken into account in this procedure, since unconsciousness was immediately induced after stunning in these animals.

Differences were considered to be significant at the $5 \%$ probability level.

\section{RESULTS}

\section{Animals}

The total number of calves used in this study was 82 . In one calf from treatment 4 , the EEG signal was lost after stunning and consequently assessment of loss of consciousness was impossible. Data from this calf was excluded from further analyses.

\section{Electroencephalogram activity}

A clear EEG signal was obtained from all calves during baseline recordings. Based on EEG recordings, calves stunned with a captive bolt (treatment 1 ) were unconscious $1 \pm$ $0 \mathrm{~s}$ after stunning. No transitional stage was observed in these calves. One calf, however, lost consciousness $11 \mathrm{~s}$ after stunning based on EEG recordings. Minimal brain activity was observed in all calves from $22 \pm 19 \mathrm{~s}$ after stunning. Calves of treatments 2 and 3 developed a transitional EEG based on EEG recordings $54 \pm 13$ and $27 \pm 15$ s, respectively, after the neck cut. Calves of treatments 2 and 3 were considered unconscious based on EEG recordings $109 \pm 32$ and $49 \pm 25$ s, respectively, after the neck cut. Calves of treatments 2 and 3 had minimal brain activity based on EEG recordings $157 \pm 50$ and 86 $\pm 34 \mathrm{~s}$, respectively, after the neck cut.

Figure 3 represents the percentage of calves displaying different EEG stages over time subjected to non-stunned slaughter in an upright position (treatment 2) or an inverted position (treatment 3 ) at $\mathrm{T}=0 \mathrm{~s}$. The time span for $80 \%$ or $100 \%$ of the calves to reach a transitional EEG, unconsciousness or minimal brain activity is displayed in Table 1. Eighty percent of the calves slaughtered in an upright position (treatment 2) lost consciousness on average $61 \mathrm{~s}$ later than calves slaughtered in an inverted position (treatment 3). All the calves slaughtered in an upright position (treatment 2) lost 
consciousness on average $31 \mathrm{~s}$ later than calves slaughtered in an inverted position (treatment 3).

Of the treatment 2 calves, $20 \%$ had a transitional EEG, none were considered unconscious and none had minimal brain activity based on EEG recordings $40 \mathrm{~s}$ after the neck cut. Of the treatment 3 calves, $81 \%$ of the calves had a transitional EEG, $46 \%$ were considered unconscious and $8 \%$ had minimal brain activity based on EEG recordings $40 \mathrm{~s}$ after the neck cut.

Treatment 4 calves (non-stunned slaughter in an upright position followed by captive bolt stunning) were stunned on average $34 \pm 8 \mathrm{~s}$ after the neck cut. At stunning, $67 \%$ of the calves were conscious, $8 \%$ of the calves had a transitional EEG, $21 \%$ of the calves were unconscious and $4 \%$ of the calves displayed minimal brain activity. All calves that were conscious or had a transitional EEG before stunning were unconscious $1 \pm 0 \mathrm{~s}$ after stunning based on EEG recordings.
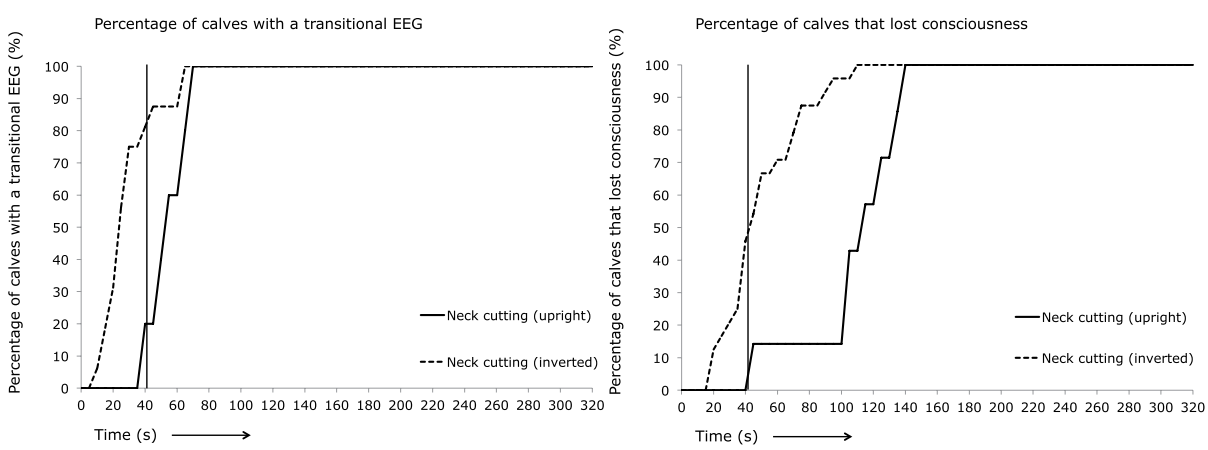

A

B

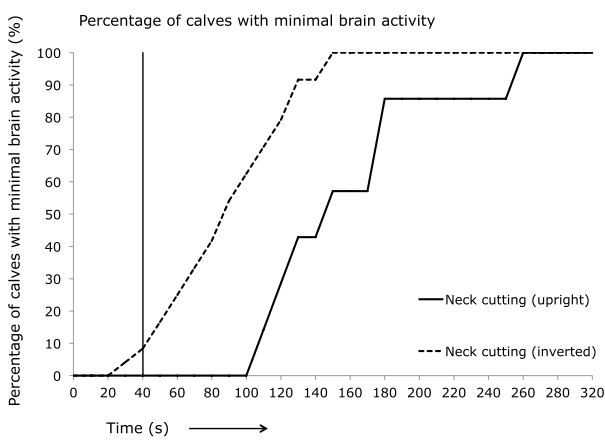

C

\section{FIGURE 3A-C}

Percentage of calves with different electroencephalogram (EEG) stages through time after non-stunned slaughter in an upright position (red lines, $n=7$ ) or inverted position (blue lines, $n=25$ ) at $\mathrm{T}=0 \mathrm{~s}$. Vertical black line represents the percentage of calves with a specific EEG stage at $40 \mathrm{~s}$. 
TABLE 1

Time points at which 80 or $100 \%$ of the calves subjected to non-stunned slaughter $(\mathrm{T}=0 \mathrm{~s})$ in an upright $(n=7)$ or inverted position $(n=25)$ had a specific electroencephalogram (EEG) stage.

\begin{tabular}{|c|c|c|c|c|c|c|}
\hline \multirow{3}{*}{$\begin{array}{l}\text { Percentage of } \\
\text { animals with a } \\
\text { specific } \\
\text { EEG-stage }\end{array}$} & \multirow{2}{*}{\multicolumn{3}{|c|}{$\begin{array}{l}\begin{array}{l}\text { Non-stunned slaughter in an upright } \\
\text { position }\end{array} \\
\text { Start of EEG-stage (s) }\end{array}$}} & \multirow{2}{*}{\multicolumn{3}{|c|}{$\begin{array}{l}\text { Non-stunned slaughter in an inverted } \\
\text { position }\end{array}$}} \\
\hline & & & & & & \\
\hline & 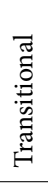 & 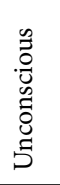 & 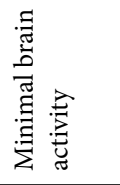 & 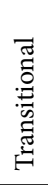 & 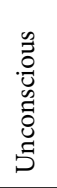 & 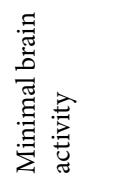 \\
\hline $80 \%$ & 62 & 132 & 175 & 36 & 71 & 122 \\
\hline $100 \%$ & 70 & 140 & 253 & 62 & 109 & 147 \\
\hline
\end{tabular}

\section{Loss of reflexes for unconsciousness}

Reflexes were tested and present in all calves during baseline recordings. In calves of treatment 1, threat-, withdrawal-, corneal-, and eyelid reflex were all permanently lost at the first testing moment ( $\mathrm{T}=5 \mathrm{~s}$ ) after captive bolt stunning. Figure $4 \mathrm{a}$ presents the range of individual time points at which the different reflexes were lost in relation to onset of the different EEG stages ( $\mathrm{T}=0 \mathrm{~min}$ ), based on visual assessment of EEG recordings during non-stunned slaughter of calves in an upright position (treatment 2). In all treatment 2 calves, the threat-, and withdrawal reflex were both lost before EEG-based loss of consciousness had been observed. The corneal-, and eyelid reflex were both lost in all calves of treatment 2 after EEG-based loss of consciousness was observed. Corneal-, and eyelid reflex were lost in $71 \%$ and $86 \%$ respectively, of calves of treatment 2 after onset of minimal brain activity.

Figure $4 \mathrm{~b}$ represents the range of individual time points at which the different reflexes were lost in relation to onset of the different EEG stages ( $\mathrm{T}=0 \mathrm{~min}$ ), based on visual assessment of EEG recordings during non-stunned slaughter of calves in an inverted position (treatment 3 ). In calves of treatment 3 , the threat-, and withdrawal reflex were lost in $88 \%$ and $92 \%$ respectively, of the animals before EEG-based loss of consciousness was observed. Both corneal-, and eyelid reflex were lost in $92 \%$ of calves of treatment 3 after EEG-based loss of consciousness had been observed. The threat-, and withdrawal reflex were lost in $92 \%$ and $96 \%$ respectively, of calves of treatment 3 before minimal brain activity was observed. The corneal-, and eyelid reflex were lost in $88 \%$ and $67 \%$ respectively, of calves of treatment 3 after minimal brain activity had been observed. Calves of treatments 2 and 3 lost the corneal reflex $235 \pm 86$ and $123 \pm 34$ s, respectively, after the neck cut. Calves of treatments 2 and 3 lost the eyelid reflex $218 \pm 65$ and $115 \pm$ $36 \mathrm{~s}$, respectively, after the neck cut. 

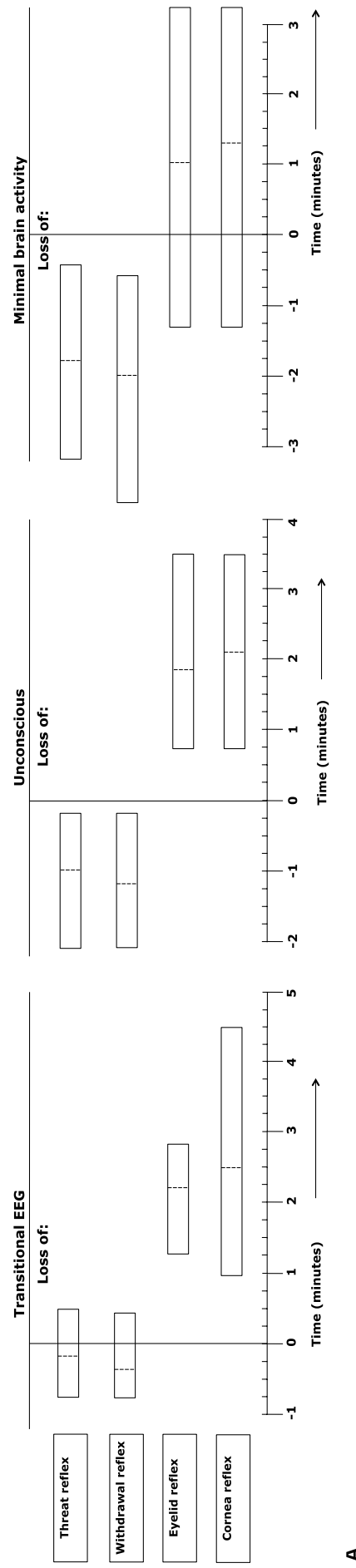
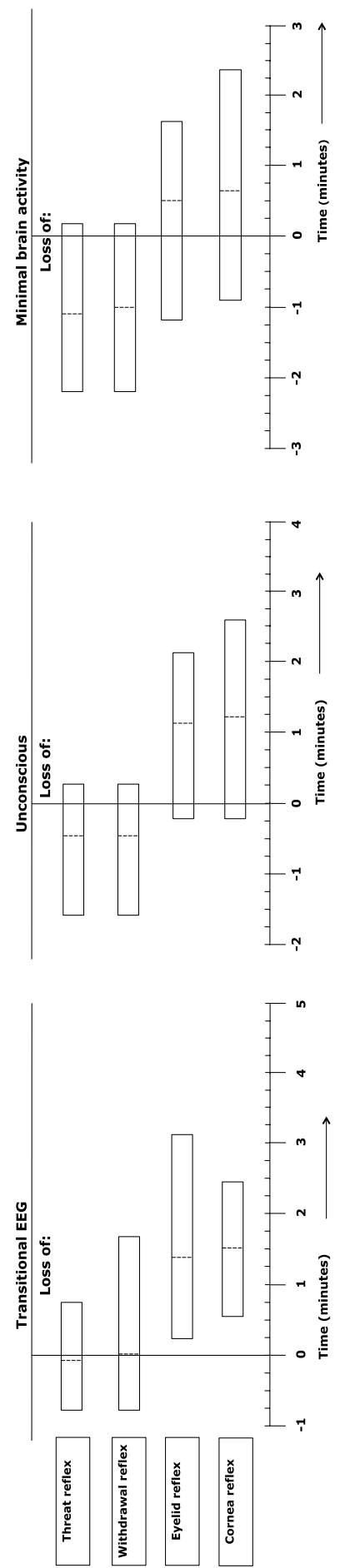

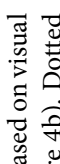
总高. 寻造密

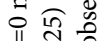
도을

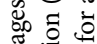
进 (⿹ 武朖 हี b. 을 ส क्षे च्ञ 范 䓀 $\approx$ 苞会苛 氖 II 㱐 氜 : 氜 造 을 츨 ॠ

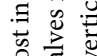
兽

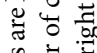

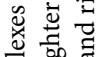
政

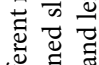
可 娄 氠

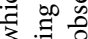
可

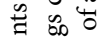
$\exists$. 氞 毛 泀式苟 $\infty$ 空

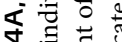
山苟 司

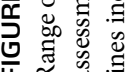


All calves of treatment 4, lost both the threat-, or withdrawal reflex before EEG-based loss of consciousness. The corneal-, or eyelid reflex were lost in $89 \%$ and $87 \%$ respectively of the calves after EEG-based loss of consciousness. The threat-, withdrawal-, corneal-, and eyelid reflex were all permanently lost $5 \mathrm{~s}$ after the captive bolt stunning.

\section{DISCUSSION}

The main aim of this study was to provide insight into the validity of certain reflexes that are used to assess unconsciousness at slaughter in veal calves, that is the threat-, withdrawal-, corneal-, and eyelid reflex. Brain activity, as presented in an electroencephalogram or EEG, served as a 'gold standard' for our assessment of unconsciousness.

Consciousness involves the cerebral cortex and thalamus, together forming the thalamocortical complex, which is regulated by the brainstem. The central core of the brainstem is formed by the reticular formation, a large network of neural tissue located in the central region of the brain stem. Neuronal projections from the reticular formation to the cortex are called the ascending reticular activating system (ARAS). A goodfunctioning reticular formation and ARAS are essential for the maintenance of consciousness and damage to (one of) these regions causes rapid loss of consciousness. Unconsciousness induced by penetrating captive bolt stunning is caused by a combination of the concussive force generated by impact of the bolt on the cranium and irreversible physical damage produced by passage of the bolt through the brain. Generally, the pistol is placed on the forehead after which the bolt penetrates the skull to target the brain stem. Immediate loss of consciousness and reflexes should occur from the resulting trauma to the cerebral hemisphere and brain stem (Terlouw et al., 2016a).

Depending on the method of stunning, the EEG shows a characteristic pattern of change when animals lose consciousness. Generally, an increase in low frequency activity is accompanied by an increase in amplitude. When neurons depolarise at the same time or frequency, they fire in a synchronised fashion creating slow high amplitude waves as seen in unconscious states suggesting a depression of the reticular formation (Lopes da Silva, 1982). EEG recordings in captive bolt stunned animals displayed high-amplitude, slowfrequency waves after stunning, indicating immediate unconsciousness, similar to findings of Lambooy and Spanjaard (1981) and Zulkifli et al. (2014). One calf in the current study displayed a period of baseline-like EEG activity, lasting for $11 \mathrm{~s}$ after captivebolt stunning. This calf displayed a permanent loss of all reflexes from $5 \mathrm{~s}$ after stunning. Reflexes in the conscious calf before the beginning of the slaughter procedure were always present and it is therefore unlikely that these reflexes were lost, though the calf was conscious. The prolonged period of baseline-like EEG activity may have been caused by 
incorrect positioning of the stunner, a deflected shot, incorrect functioning of the stunning device itself or an ineffective EEG recording.

Effectively shot cattle should collapse immediately due to damage to the reticular formation that also plays a role in maintaining posture (Laureys and Tononi, 2009). In our study, an immediate collapse was observed in some of the calves, but tight restraining of the calf often prevented collapse and made observation of this criterion difficult. Five seconds after captive bolt stunning all reflexes were permanently lost in all calves. Rhythmic breathing and the righting reflex are considered indicative of a (partial) return of consciousness and thus that the stunning has not been irreversible (Grandin, 2002). Both rhythmic breathing and the righting reflex could not be assessed because the animals were fully restrained in a rotatory box.

Non-stunned animals that are subjected to neck cutting lose consciousness gradually as a consequence of widespread anoxia in the brain that results in progressive dysfunction of cortical and subcortical structures (Terlouw et al., 2016a). During our experiment, calves in an inverted or upright position lost consciousness, based on EEG recordings, $49 \pm 25$ and $109 \pm 32$ s, respectively, (mean \pm SD) after the neck cut. Previous studies in cattle indicated loss of consciousness ranging from $10 \mathrm{~s}$ up to over 3 minutes after the neck cut (Daly et al., 1988; Gregory et al., 2010; Bourguet et al., 2011). Newhook and Blackmore (1982b) suggested the possibility of an intermittent return to consciousness for up to 123 to $323 \mathrm{~s}$ after non-stunned slaughter in cattle. Detailed study of EEG recordings did not indicate signs of resurgence of consciousness in any of the calves in our study. The corneal,- and eyelid reflex, however, were lost and regained in $9 \%$ and $16 \%$ respectively of the non-stunned calves $(n=32)$ during bleeding. Loss and return of the corneal reflex has been observed by Hoffman in an unspecified number of cattle (Hoffman, 1900 cited by Gregory et al., 2010). Bourguet et al. (2011) also observed a temporary return of the palpebral and corneal reflex in some of the non-stunned slaughtered cattle. A temporary return of blood pressure would be necessary for these reflexes to return and may possibly be explained by physiological changes, such as an increased heart rate, to alleviate the drop in blood pressure (Newhook \& Blackmore, 1982; Vimini et al., 1983). There is no clear indication why reflexes were regained and lost again in some of the calves in the current experiment.

Because this experiment took place at a commercial slaughter plant, the slaughter man was empowered to decide when an additional cut was necessary. No clear criteria were defined for this. In total 15 of $82(18 \%)$ calves in our study were cut a second time and one of those 15 calves was cut a third time. The arteries of an animal in which poor bleeding is observed, are in practice often re-cut to allow adequate bleeding. Poor bleeding may be caused by occlusion, which is characterised by retraction and contraction of the elastic portion of the arterial wall and thrombus formation around the severed end of the vessel (Gregory et al., 2006). The prevalence of carotid arterial occlusion in 576 cattle 
slaughtered at abattoirs in the United Kingdom was found to be $16 \%$ and 25\%, respectively, for adult cattle and bobby calves (Gregory et al., 2006). A study by Gregory et al. (2010) showed that $71 \%$ of cattle taking longer than $75 \mathrm{~s}$ to collapse, had a false aneurysm in the cardiac end of the carotid that was at least $3 \mathrm{~cm}$ in diameter. There was no further investigation into the background of this obstruction, but it may provide an explanation for the large variation in time to loss of consciousness between calves.

Duration to loss of consciousness was $50 \mathrm{~s}$ longer in non-stunned veal calves restrained upright than by those restrained in an inverted position. During this experiment, all calves were slaughtered in the same rotation box which normally is used for inverted slaughter. Hence, slaughter using a box designed for upright restraint may provide different results. Blood flow in calves slaughtered upright was often obstructed when the head fell limp against the head restrainer. Based on this observation and in consideration of the potential risk to animal welfare, the number of veal calves slaughtered non-stunned in an upright position was limited to seven calves. Inversion of cattle for slaughter in itself is widely debated and thought to cause distress because of a longer delay between entering the restrainer and full restraint. In addition there is discomfort from the inverted position resulting in hypoxemia and rumen pressure on the diaphragm (Dunn, 1990; Petty et al., 1994; Tagawa et al., 1994). Another potential welfare problem is aspiration of blood and refluxing gut content after neck cutting in non-stunned animals (EFSA, 2004). Though the latter problem is mainly associated with the inverted position, it has also been observed in non-stunned slaughtered calves in the upright position (Grandin and Regenstein, 1994; Gregory et al., 2009b). A report by the Farm Animal Welfare Committee (2012) stated that there is little evidence of any welfare advantages of inversion in terms of speed and efficiency of the cut. Visits to 18 European cattle slaughter plants showed that the mean number of cuts was higher for cattle restrained in an upright position than those in other restraining systems (Dialrel, 2010). Although, others claim, that the angle of the head in inverted cattle allows for a more effective downward cut compared to the upward cut in animals in an upright position (Slaughter of Animals (prevention of cruelty) Regulations 1958 - quoted by Dunn, 1990). The present study is, to our knowledge, the first to suggest that the neck cut may be more effective in terms of duration to loss of consciousness in non-stunned slaughtered animals in an inverted position.

Reflexes in non-stunned slaughtered animals were not lost immediately, as in captive bolt stunned animals, but disappeared gradually. Generally, the threat reflex and withdrawal reflex were lost first, followed by the corneal- and eyelid reflex. Loss of reflexes in this order may be dependent on the difference in resistance to anoxia by different parts of the brain. Functions that rely on integration of cortical processes are more susceptible to anoxia than those originating from lower brain structures, such as the brain stem (Hansen, 1985). On average, the threat reflex was lost in non-stunned calves before EEG-based 
unconsciousness. The rapid loss of the threat reflex can possibly be explained by the sudden drop in blood flow after neck cutting causing hypoxia of the brain cells involved (Bourguet et al., 2011). A similar phenomenon was also observed for the withdrawal reflex that was lost on average before EEG-based loss of consciousness. When the neck is cut, the knife transects veins, arteries and other blood vessels, skin, muscle, trachea, oesophagus, sensory- and motor nerves, and connective tissue. Transecting these soft tissues will send a considerable amount of neural impulses to the brain (Gregory, 2008a). The massive stimulation of all sensory nerves after the neck cut may overrule the neural input from other, milder sources of pain, such as pinching the nose. Opinions on pain perception during neck cutting are divided. Some (e.g. Grandin, 1994; Rosen, 2004) state that use of an exquisitely sharp knife, will prevent pain after the neck cut. Others (e.g. Gibson et al., 2007; Gibson et al., 2009a; Mellor et al., 2009) state that the ventral neck cut is a noxious stimulus and that there is strong evidence that this cut would be perceived as painful from the time of the neck cut until loss of consciousness. Until now, neurophysiological methodology has not provided a final and definitive answer to this issue.

Both the corneal- and eyelid reflex require a functional afferent cranial nerve $\mathrm{V}$ (trigeminal) and efferent cranial nerve VII (facial) and the relevant eye muscles to function adequately (Adams and Sheridan, 2008). The connections between the spinal trigeminal- and facial nuclei pass through the reticular formation and failure of these reflexes often indicates a wider dysfunction of the brain, comprising part of the reticular formation, and thus a state of unconsciousness (Terlouw et al., 2016a,b). In the present study, the corneal reflex in non-stunned slaughtered calves was lost on average $85 \pm 45 \mathrm{~s}$ after EEG-based loss of consciousness and on average $47 \pm 63 \mathrm{~s}$ after minimal brain activity was observed. Lambooij et al. (2012) and Newhook and Blackmore (1982b) observed that non-stunned slaughtered calves lost the corneal reflex up to 1 minute after calves were unconscious (EEG-based) and this reflex was even observed in some calves after all EEG activity had been lost. Verhoeven et al. (2015b) observed that non-stunned slaughtered sheep lost the corneal reflex on average 00:59 \pm 00:17 min (mean \pm SD) after sheep were considered to be unconscious based on EEG recordings. The eyelid reflex in non-stunned slaughtered calves was lost on average $76 \pm 50 \mathrm{~s}$ after EEG-based loss of consciousness and $37 \pm 59 \mathrm{~s}$ after minimal brain activity was observed. Other studies report the presence of an eyelid reflex in unconscious sheep (Verhoeven et al., 2015b) and cattle (Newhook and Blackmore, 1982a) and also during anaesthesia. The eyelid reflex is known to be present after an animal is considered unconscious (Dugdale, 2010). The order in which the eyelid- and corneal reflex cease may differ, providing uncertainty which reflex is more resistant to the effects of anoxia (Bourguet et al., 2011; Terlouw et al., 2016b).

The results from this study support the hypothesis that a positive corneal- or eyelid reflex alone does not necessarily indicate consciousness, since positive brain stem reflexes might occur on the basis of residual brain stem activity and do not distinguish clearly between 
consciousness and unconsciousness (Anil, 1991). Consequently, a negative corneal- or eyelid reflex is considered a general indication of unconsciousness (Laureys, 2005; Terlouw et al., 2016b).

\section{CONCLUSIONS}

In both stunned and non-stunned calves, unconsciousness was induced and absence and presence of reflexes was successfully linked to EEG activity. The use of reflexes to assess unconsciousness can more easily be applied in daily practice, and EEG recordings can be used to validate the interpretation of such reflexes. After captive bolt stunning, absence of threat-, withdrawal,- corneal,- and eyelid reflex indicated unconsciousness as determined by EEG recordings. On average, after non-stunned slaughter, the threat- and withdrawal reflex were lost before calves were considered unconscious based on EEG recordings. The eyelid- and corneal reflex were on average lost after calves were considered unconscious based on EEG recordings and appeared to be distinctly conservative indicators of unconsciousness in non-stunned slaughtered calves since they were observed up to $76 \pm 50$ and $85 \pm 45 \mathrm{~s}$ (mean $\pm \mathrm{SD}$ ) respectively after EEG-based onset of unconsciousness.

\section{ACKNOWLEDGEMENTS}

The authors would like to thank Vincent Hindle and Samantha Surquin for their support while collecting the data. Jan Peuscher is acknowledged for his technical assistance while collecting the data and his help during data analyses. The authors also acknowledge the owner and employees of the slaughter plant for their assistance and hospitality during the study. Funding for this project was provided by the Dutch Ministry of Economic Affairs (KB-12-006.01-002). 
ASSESSING UNCONSCIOUSNESS IN VEAL CALVES | 83 
Frontiers in Veterinary Science (2016) 3 (38): 1-10

DOI: $10.3389 /$ fvets.2016.00038

M.T.W. Verhoeven ${ }^{1,2}$, M.A. Gerritzen ${ }^{1}$, A. Velarde ${ }^{3}$, L.J. Hellebrekers ${ }^{4}$, and B. Kemp ${ }^{2}$

${ }^{1}$ Animal Welfare Department, Animal Sciences Group, Wageningen University and Research Centre, Wageningen, The Netherlands

${ }^{2}$ Adaptation Physiology Group, Animal Sciences Group, Wageningen University,

Wageningen, The Netherlands

${ }^{3}$ Animal Welfare Subprogram, IRTA, Veïnat de Sies s/n, 17121, Monells, Girona, Spain

${ }^{4}$ Central Veterinary Institute, Animal Sciences Group, Wageningen University and Research

Centre, Lelystad, The Netherlands 
TIME TO LOSS OF CONSCIOUSNESS AND ITS RELATION TO BEHAVIOUR IN SLAUGHTER PIGS DURING STUNNING WITH 80\% OR 95\% CARBON DIOXIDE

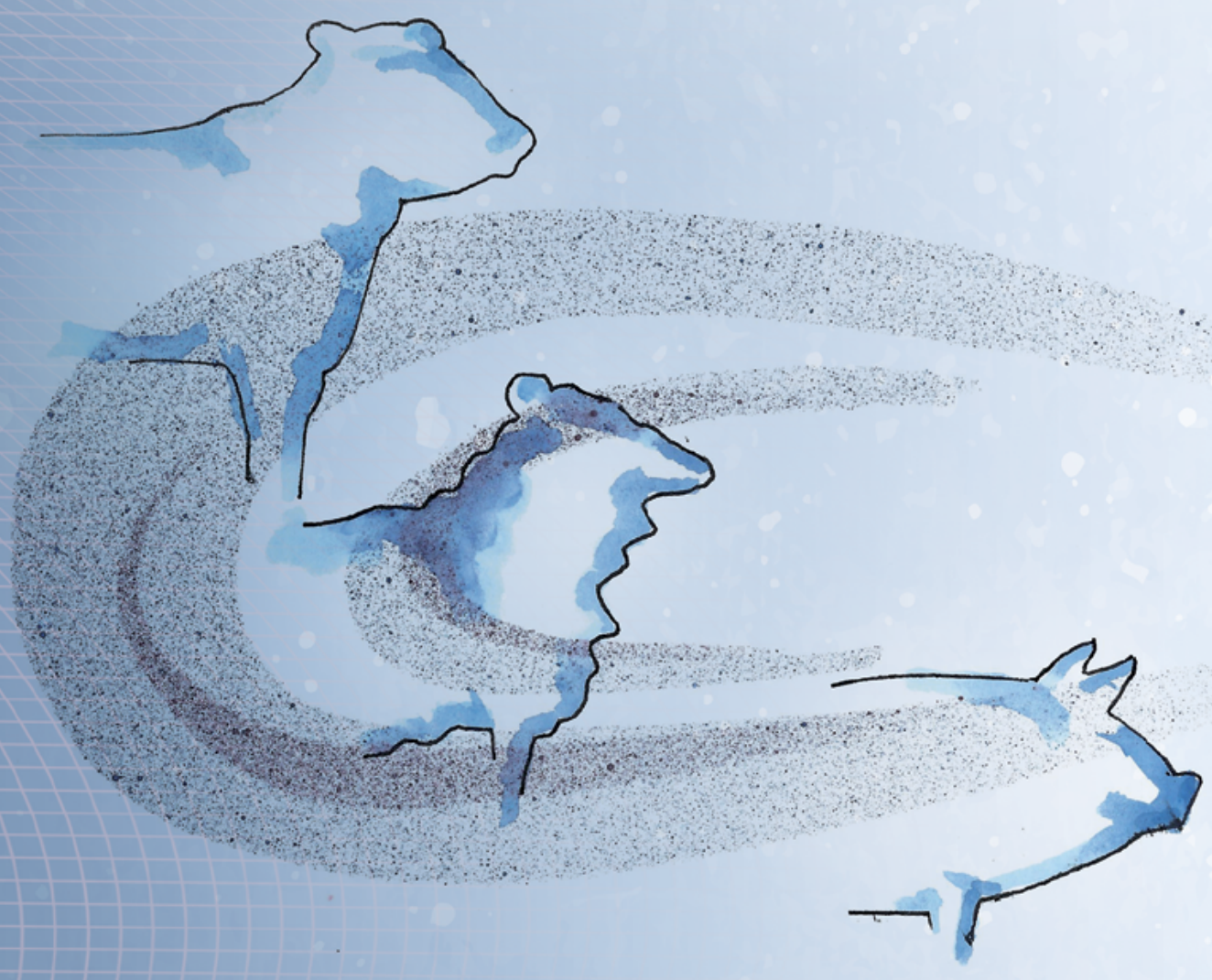




\section{ABSTRACT}

Exposure to $\mathrm{CO}_{2}$ at high concentration is a much debated stunning method in pigs. Pigs respond aversively to high concentrations of $\mathrm{CO}_{2}$ and there is uncertainty about what behaviours occur before and after loss of consciousness. The aim was to assess timing of unconsciousness in pigs during exposure to high concentrations of $\mathrm{CO}_{2}$ based on changes in electroencephalogram (EEG) activity and the relation with the behaviours sniffing, retreat- and escape attempts, lateral head movements, jumping, muscular contractions, loss of posture, and gasping. Pigs ( $108 \pm 9 \mathrm{~kg}$ ) were randomly assigned to $80 \% \mathrm{CO}_{2}(80 \mathrm{C}$, $n=24)$ or $95 \% \mathrm{CO}_{2}(95 \mathrm{C}, n=24)$. The time at which the gondola started descending into the well pre-filled with $80 \mathrm{C}$ or $95 \mathrm{C}$ was marked as $\mathrm{T}=0$. The $\mathrm{CO}_{2}$ exposure lasted $346 \mathrm{~s}$ after which the corneal reflex and breathing were assessed for one minute. Visual assessment of changes in the amplitude and frequency of EEG traces after $\mathrm{T}=0$ was used to determine loss of consciousness. Time to loss of consciousness was longer in $80 \mathrm{C}$ pigs $(47 \pm 6 \mathrm{~s})$ than in $95 \mathrm{C}$ pigs ( $33 \pm 7 \mathrm{~s}$ ). Time to an iso-electric EEG was similar in $80 \mathrm{C}$ pigs ( $75 \pm 23 \mathrm{~s}$ ) and $95 \mathrm{C}$ pigs ( $64 \pm 32 \mathrm{~s}$ ). When pigs descended into the well, the earlier entry of $95 \mathrm{C}$ pigs into high $\mathrm{CO}_{2}$ atmosphere rather than the concentration of $\mathrm{CO}_{2}$ by itself affected the latency of behavioural responses and decreasing brain activity. During exposure to the gas, $80 \mathrm{C}$ and $95 \mathrm{C}$ pigs exhibited sniffing, retreat attempts, lateral head movements, jumping and gasping before loss of consciousness. 95C pigs exhibited all these behaviours on average earlier than $80 \mathrm{C}$ pigs after $\mathrm{T}=0$. But the interval between onset of these behaviours and loss of consciousness and the duration of these behaviours, except gasping, was similar for both treatments. Loss of posture was on average observed in both groups $10 \mathrm{~s}$ before EEG-based loss of consciousness. Furthermore, 88\% of 80C pigs and $94 \%$ of $95 \mathrm{C}$ pigs demonstrated muscular contractions before loss of consciousness. The findings provide little reason to conclude on a behavioural basis that these atmospheres are greatly different in their impact on pig welfare.

\section{INTRODUCTION}

The two most commonly used stunning methods applied under commercial slaughterhouse conditions in pigs are electrical stunning and exposure to high concentrations of carbon dioxide $\left(\mathrm{CO}_{2}\right)$ (Verhoeven et al., 2016b). The $\mathrm{CO}_{2}$ stunning method involves lowering groups of pigs in a gondola into a well that is pre-filled with a high concentration of $\mathrm{CO}_{2}$. According to European legislation, the $\mathrm{CO}_{2}$ concentration should at least be $80 \%$, but many slaughterhouses use $90 \% \mathrm{CO}_{2}$ or higher in attempts to increase throughput at the slaughter plant (Velarde et al., 2007; Council Regulation (EC) $1009,2009)$. Rapid and deeper respiration induced by higher $\mathrm{CO}_{2}$ concentrations 
increases the intake of $\mathrm{CO}_{2}$ that shortens the induction period and time to loss of consciousness (Forslid, 1992). Induction of unconsciousness with $\mathrm{CO}_{2}$ stunning requires high concentrations of $\mathrm{CO}_{2}$ where excessive $\mathrm{CO}_{2}$ concentrations in the blood lead to a state of hypercapnic hypoxia, inducing a decline in blood $\mathrm{pH}$ levels. Because $\mathrm{CO}_{2}$ travels across the blood-brain barrier relatively easy, the high $\mathrm{CO}_{2}$ levels also cause rapid acidification of the cerebrospinal fluid. The drop in $\mathrm{pH}$ is detected by central chemical receptors in the medulla oblongata and pons of the brainstem, resulting in faster and deeper respiration in an attempt to increase $\mathrm{pO}_{2}$ and decrease $\mathrm{pCO}_{2}$ (Siesjö, 1972). The acidification of the brain cells results in a depression of brain activity that causes loss of consciousness or when prolonged death (Martoft et al., 2002).

Loss of consciousness is not immediate upon exposure to high $\mathrm{CO}_{2}$ levels, but depends on the $\mathrm{CO}_{2}$ concentration used and the speed at which animals are immersed into the highest concentration of $\mathrm{CO}_{2}$ at bottom of the well (Troeger, 1991, Raj and Gregory, 1996). Time to loss of posture, as the first indicator of the onset of unconsciousness, was reported at 25,17, 22 and $15 \mathrm{~s}$ after immersion into $60,70,80$ and $90 \% \mathrm{CO}_{2}$, respectively (Raj and Gregory, 1996). Studies that examine brain activity, presented in an electroencephalogram (EEG), reported loss of consciousness 14-60 s after initial exposure to $80-90 \% \mathrm{CO}_{2}$ (EFSA, 2004; Rodriguez et al., 2008). Pigs do not need to be individually restrained and can be stunned in groups during $\mathrm{CO}_{2}$ stunning, which are considered to be advantages in terms of animal welfare in comparison to other stunning methods. (Velarde et al., 2000; EFSA, 2004). Before pigs lose consciousness, however, behaviour including excitement, retreat and escape attempts and respiratory changes (gasping) have been observed (Raj and Gregory, 1995 and 1996; Velarde et al., 2007; Terlouw et al., 2016). Carbon dioxide itself causes irritation of nasal mucosal membranes and is a strong respiratory stimulator that induces a sense of breathlessness prior to loss of consciousness in humans (Manning and Schwartzstein, 1995; Raj, 2006). Beausoleil and Mellor (2015) describe three different traits of breathlessness, namely: chest tightness, respiratory effort and air hunger, where air hunger occurs when the demand for ventilation exceeds the capacity to provide it (Parshall et al., 2012). This air hunger may occur when an animal continuously inhales high tensions of $\mathrm{CO}_{2}$ and is a serious concern for animal welfare as it always unpleasant to the animal (Beausoleil and Mellor, 2015).

Furthermore, there is much debate about what $\mathrm{CO}_{2}$ concentrations are most aversive to pigs since behavioural responses of pigs vary with different $\mathrm{CO}_{2}$ concentrations. Pigs seem to respond less aversive to lower concentrations of $\mathrm{CO}_{2}(50-60 \%)$ than higher concentrations of $\mathrm{CO}_{2}$ (80-90\%) (Dodman, 1976; Raj and Gregory, 1996; Holst, 2002). When looking at these high concentrations of $\mathrm{CO}_{2}$, Nowak et al. (2007) observed higher lactate levels, indicative of stress, in pigs exposed to $80 \% \mathrm{CO}_{2}$ compared to pigs exposed to $90 \%$ CO2. Barfod (1990) and Erhardt et al. (1989) on the other hand did not find conclusive evidence that $\mathrm{CO}_{2}$ irritates the membranes and concluded that gasping is a 
normal response to the excessive $\mathrm{CO}_{2}$ in the body. The general opinion, however, is that the initial phase of $\mathrm{CO}_{2}$ stunning is aversive to pigs (Nowak et al., 2007).

While changes in breathing pattern are generally associated with aversion, there is little consensus concerning the interpretation of the occurrence of convulsions or (involuntary) muscle contractions (Terlouw et al., 2016b). These muscle contractions have been observed both before (Velarde et al., 2007; Rodriguez et al., 2008) and after loss of consciousness (Hoenderken, 1983; Forslid, 1987; Velarde et al., 2007). The objective of the current study was to assess the relationship between behavioural measurements and onset of unconsciousness as identified by EEG activity during $80 \% \mathrm{CO}_{2}$ (80C) or $95 \%$ $\mathrm{CO}_{2}$ (95C) stunning in pigs.

\section{MATERIALS AND METHODS}

This study was approved by the Animal welfare body of Wageningen UR, The Netherlands and by the Institutional Animal Care and Use Committee (IACUC) of IRTA, Spain.

\section{Animals and housing}

In total, 48 cross breed (Pietrain $\mathrm{x}$ Large White $\mathrm{x}$ Landrace) pigs from a commercial fattening farm were randomly selected and transported to the experimental facilities. Before departure from the farm, all animals were systematically inspected by clinical examination of physical appearance and the normality of behaviour, removing those presenting signs of disease. The selected pigs (live weight $108 \pm 9 \mathrm{~kg}$ ) arrived at the experimental facilities 3 days prior to start of the experiment and were housed in groups of eight in six adjacent lairage pens of $4.5 \mathrm{~m} \mathrm{x} 1.8 \mathrm{~m}$, next to the experimental abattoir. The pigs had free access to water and were fed ( $3 \mathrm{~kg} / \mathrm{pig} /$ day) twice daily at $0700 \mathrm{~h}$ and $1600 \mathrm{~h}$ using the same commercial diet they received on the fattening farm.

\section{Experimental set-up}

From the day of arrival until the beginning of the experiment, pigs were habituated to human contact twice a day for 5 min per pen. The experiment was conducted on four consecutive days, starting 3 days after arrival of the pigs. The $\mathrm{CO}_{2}$ stunning unit was a dip-lift system (Butina Aps, Copenhagen, Denmark) that contained a gondola $(299 \mathrm{~cm}$ $\times 138 \mathrm{~cm} \times 100 \mathrm{~cm}$ ) which descended to the base of a well at a depth of $290 \mathrm{~cm}$. On the first 2 days, pairs of pigs (always the same pairs randomly selected from the same lairage pen) were habituated to the ascending and descending of the dip-lift (once every day) containing atmospheric air. All pigs were equipped with EEG electrodes and a respiratory band each day, before they entered the gondola. In order to confirm that there was no effect of being in a (ascending or descending) gondola on EEG activity, data were recorded 
in 24 of the 48 pigs (one pig from each pair). The descent of the gondola took $23 \mathrm{~s}$, where it remained at the bottom of the well for $30 \mathrm{~s}$ before ascending in $23 \mathrm{~s}$. The total cycle lasted $76 \mathrm{~s}$ and when the gondola reached the top, the pigs were allowed to exit the gondola and the recording equipment was removed. Thereafter, pigs were allowed to return to their pen.

On the third or fourth day, the same pairs of pigs, equipped with EEG electrodes and a respiratory band were again placed in the dip-lift gondola and exposed to the stunning treatments. The well was pre-filled with $\mathrm{CO}_{2}$ through an inlet valve at the bottom of the well and the $\mathrm{CO}_{2}$ concentration was pre-set and measured using a sensor placed at a depth of $2.5 \mathrm{~m}$. After the complete experiment had finished, $\mathrm{CO}_{2}$ concentrations were measured once at five different depths into the well while the well was pre-filled with $80 \mathrm{C}$ or $95 \mathrm{C}$. The gondola contained $80 \mathrm{C}$ on the first morning and second afternoon and $95 \mathrm{C}$ on the first afternoon and second morning of day 3 and 4 . Descent of the gondola took $23 \mathrm{~s}$, before remaining stationary at the bottom for $300 \mathrm{~s}$ before ascending in $23 \mathrm{~s}$. The total cycle lasted $346 \mathrm{~s}$ and when the gondola reached the top, the exit gate was opened and pigs were assessed for signs of return to consciousness. The corneal reflex was assessed at $10 \mathrm{~s}$ intervals and occurrence of breathing was assessed continuously for $60 \mathrm{~s}$. Thereafter, the EEG electrodes and the respiratory band were removed and each pig was bled and sent for further processing.

\section{Electroencephalogram activity and respiratory signal measuring procedure}

To facilitate instrumentation pigs were fixated in a standing position in a weighing scale (Figure 1a). A nose clamp or any other additional restraining method was not required.

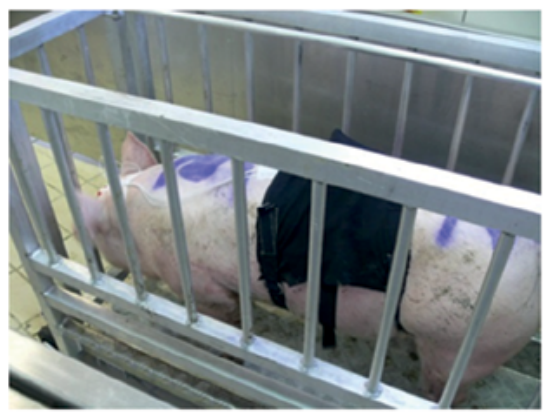

A

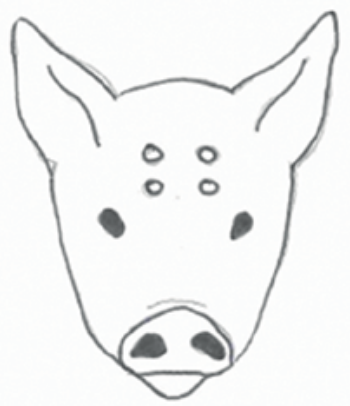

B

FIGURE 1A,B

Weighing scale in which the pigs were fixated (Figure 1a) to equip them with EEG-recording equipment and a respiratory band. Placement of the four electrodes on the pigs head (Figure 1b). 
During instrumentation pigs remained in the weighing scale for approximately $10 \mathrm{~min}$. The head of the animal was shaved on day 1 with an electrical trimmer to enable placement of EEG electrodes. Four Ag/Cl electrodes (Twente Medical Systems International (TMSi), Oldenzaal, The Netherlands) were placed on the shaved skin after applying adhesive tissue ( $3 \mathrm{M}$ Vetbond ${ }^{\mathrm{TM}}$, St. Paul, ME, USA). Two electrodes were placed on the skin of the forehead, $2 \mathrm{~cm}$ left and right from the sagittal midline and $2 \mathrm{~cm}$ below a line extending between the base of both ears. The other two electrodes were placed on the frontal bone $2 \mathrm{~cm}$ left and right from the sagittal midline $3 \mathrm{~cm}$ frontal from the first electrodes (Figure $1 \mathrm{~b}$ ). All electrodes were connected via a $140 \mathrm{~cm}$ active protected cable to a 6-channel Mobi system (TMSi, Oldenzaal, The Netherlands).

The Mobi system uses bipolar amplifier technology with high input impedance (> $1 \mathrm{G} \Omega$ ) that amplifies the potential difference between each pair of electrodes. The input amplifier is dimensioned as multichannel instrumentation amplifier. Electrode impedance was $<5$ $\mathrm{k} \Omega$. The EEG was displayed with a band pass filter of 0.5 and $30 \mathrm{~Hz}$, respectively, and unfiltered data were saved onto a computer. Sampling rate was set at $1 \mathrm{kHz}$. Once the electrodes had been secured and a good live signal was obtained in the weighing scale, baseline EEG activity was recorded for at least $2 \mathrm{~min}$. The gondola started descending into the well at $\mathrm{T}=0$. Recording of the EEG was continuous until the pig left the gondola (days 1 and 2) or was bled (day 3 or 4 ).

A respiratory waveform was recorded continuously by placing an inductive respiratory band $(80 \mathrm{~cm})$ around the abdomen behind the pig's last rib (TMSi, Oldenzaal, The Netherlands).

\section{Behavioural measurements}

Pig behaviour in the gondola was recorded using two video cameras (Sony Colour CD AVC 565, Circontrol, Barcelona, Spain) placed on the top of the gondola and were connected to a digital image recorder (VDVR-4S 550430, Circontrol).

After at least two minutes of baseline recording in the weighing scale, pigs were gently moved to the gondola. After the gondola, containing two pigs, descended into the well $(\mathrm{T}=0)$ number (events), duration (states) and latency to the behaviours as defined in Table 1 were scored per pig from the video recordings using Observer 5.0 software (Noldus Information technology B.V., Wageningen, The Netherlands). 


\section{TABLE 1}

Ethogram used to score the behaviours of pigs in an ascending and descending gondola into a well filled with atmospheric air on days 1 and 2 and $80 \%$ or $95 \%$ carbon dioxide $\left(\mathrm{CO}_{2}\right)$ on day 3 and 4 .

\begin{tabular}{|c|c|}
\hline $\begin{array}{l}\text { Behaviour } \\
\text { (event) }\end{array}$ & Description \\
\hline Sniffing & $\begin{array}{l}\text { Sniffing while lifting the head and considered a first sign of the pig becoming } \\
\text { aware of the } \mathrm{CO}_{2} \text {. }\end{array}$ \\
\hline Retreat attempts & Pigs backing away (Dodman, 1976). \\
\hline Gasping & $\begin{array}{l}\text { A very deep breath through a wide open mouth which may involve stretching } \\
\text { of the neck (Gregory, 2008a). }\end{array}$ \\
\hline Escape attempts & $\begin{array}{l}\text { Pigs running across the gondola and/or raising their forelegs on the side wall } \\
\text { of the gondola (Forslid, 1992). }\end{array}$ \\
\hline Jumping & Jumping in air or against the wall of the gondola \\
\hline Lateral head movements & $\begin{array}{l}\text { Head movements to the side while convulsive expulsion of air from } \\
\text { the lungs through the nose and mouth (Zeller et al., 1987). }\end{array}$ \\
\hline Muscular contractions & $\begin{array}{l}\text { Defined as a period of struggling ranging from fairly vigorous running and } \\
\text { movements to clonic convulsive seizures (Dodman, 1976). }\end{array}$ \\
\hline Loss of posture & The pig is in a recumbent position with total loss of control of posture \\
\hline $\begin{array}{l}\text { Behaviour } \\
\text { (state) }\end{array}$ & Description \\
\hline Standing & $\begin{array}{l}\text { The pig is in an upright position, without moving, with all } 4 \text { paws on the } \\
\text { floor }\end{array}$ \\
\hline Walking & The pig moves in a forward direction \\
\hline Sitting & The pig is in a sitting position \\
\hline Lying & $\begin{array}{l}\text { The pig is in a recumbent position, still having (partially) control of posture } \\
\text { (it may lift the head) }\end{array}$ \\
\hline
\end{tabular}

\section{Data analyses}

All EEG data were displayed, stored and analysed using PolyBench software (TMSi, Oldenzaal, The Netherlands). The EEG activity (amplitude and frequency) of each pig was visually assessed to determine robust changes in the individual stages i.e. baseline, unconscious and minimal brain activity. The baseline stage consisted of a low-amplitude, high frequency signal, indicating alert pigs (Figure 2a) (Verhoeven et al., 2015b, Verhoeven et al., 2016a). When high-amplitude, low frequency dominated the EEG trace this was interpreted to indicate unconsciousness (Figure 2b) (Baars et al., 2003; Verhoeven et al., 2015b; Verhoeven et al., 2016a). Minimal brain activity was reflected by a flat signal ( $<10 \%$ of baseline amplitude) (Figure 2c) (Verhoeven et al., 2015b, 2016a). Latency to first apnea was defined as the first time the respiratory waveform signal was flat for at least five seconds. Respiratory arrest was defined as the time at which the respiratory waveform signal stayed flat until the end of the experiment. 


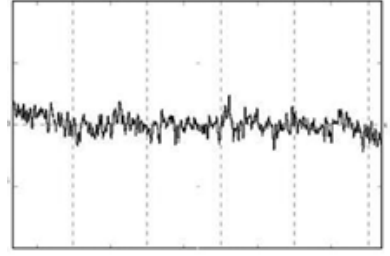

A

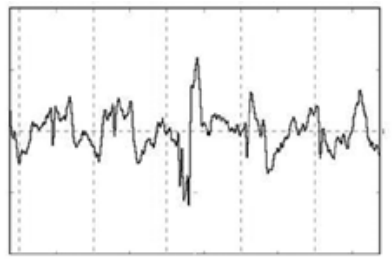

B

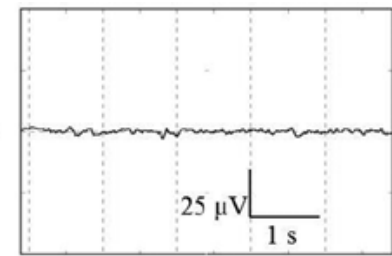

C

\section{FIGURE 2A-C}

Representative examples of the different stages identified with visual assessment of electroencephalogram (EEG) activity prior to and after $\mathrm{CO}_{2}$ stunning in pigs. The three stages from left to right: baseline (a), unconscious (b), and minimal brain activity (c). Total X-axis represents 5 seconds, Y-axis represents amplitude of the EEG-trace $(\mu \mathrm{V})$.

\section{Statistical analyses}

Behaviours and EEG variables were analysed in SAS 9.3 (SAS Inst. Inc. Cary, NC, USA). To assess whether behaviour differed between the two days during which the gondola only contained atmospheric air, the following behaviours: walking, standing and lying expressed as percentages, were analysed with either PROC MIXED when normality was approximated (standing, lying) or PROC GLIMMIX when normality could not be approximated (walking). In PROC GLIMMIX a binomial distributions with the default logit link was used. As behaviours were assessed on 2 days for each animal, observations cannot be considered independent. Therefore, a repeated measurement analysis was performed with animals as the subject applying a first order auto-regressive $[\mathrm{AR}(1)]$ variance-covariance structure determined to be the best fit according Akaike's corrected information criterion. The model included the fixed class effect of day (days 1 and 2) and the random effect of the experimental unit pair (pair 1 to 24) nested within days (model 1).

To assess effects of $\mathrm{CO}_{2}$ treatment and possible day effects on latencies to, and durations of, behaviours, standing, walking, sitting, lying, sniffing, retreat and escape attempts, gasping, lateral head movements, jumping, loss of posture and muscular contractions were analysed using PROC MIXED. The fixed class effect of treatment (80C, 95C), day (day 3,4$)$, and their interaction were assessed. A random effect of the experimental unit pair nested within day and treatment was added to the model (model 2). For retreat attempts, lateral head movements and jumping, normality was not attained and the natural logarithm of the respective variables was calculated to approximate normality. To assess effects of $\mathrm{CO}_{2}$ treatment and possible day effects on the prevalence of behaviours, i.e. retreat attempts, gasping, lateral head movements, jumping and muscular contractions assessed as a binary trait, were analysed with PROC GLIMMIX with a binary distribution and a logit link. The model included the same fixed and random effects specified in model 2. Results are displayed as mean $\pm \mathrm{SD}$, unless stated otherwise. In all cases, significance was assumed at $P<0.05$. 


\section{RESULTS}

\section{Air treatment}

\section{Electroencephalogram (EEG)}

Baseline EEG activity was representative for conscious and awake animals in all pigs $(n=24)$ : high frequency, low amplitude waves as depicted in Figure 2a. No differences in EEG stadia were observed during the baseline period or descending and ascending of the gondola for pigs in the first 2 days.

\section{Behaviour}

The percentage of time spent on walking was similar $(P>0.10)$ for pigs on day $1(11 \pm$ $9 \%)$ compared to day $2(9 \pm 7 \%)$ during the $76 \mathrm{~s}$ in the gondola. The percentage of time spent on standing was also similar $(P>0.10)$ for pigs on day $1(89 \pm 9 \%)$ compared to day $2(91 \pm 7 \%)$ during the $76 \mathrm{~s}$ in the gondola. None of the pigs were observed sitting or lying and no gasping, jumping, muscular contractions or escape attempts were observed on these 2 days.

\section{$\mathrm{CO}_{2}$ treatment \\ $\mathrm{CO}_{2}$ concentration}

The average $\mathrm{CO}_{2}$ concentration at a depth of $2.5 \mathrm{~m}$ was lower $(P<0.0001)$ during the 12 runs of $80 \mathrm{C}(82 \pm 2.1 \%)$ than during the 12 runs of $95 \mathrm{C}(97 \pm 0.5 \%)$. Table 2 shows $\mathrm{CO}_{2}$ concentrations at five different depth levels in the well for the $80 \mathrm{C}$ and $95 \mathrm{C}$ treatment measured once after the experiment had taken place.

\section{TABLE 2}

$\mathrm{CO}_{2}$ concentrations measured at different levels during descend of the gondola during the two treatments (80C and 95C).

\begin{tabular}{llll}
\hline & \multicolumn{3}{c}{ Treatment $^{3}$} \\
\hline Level of the sensor $^{1}$ & Time $(\mathrm{s})^{2}$ & $80 \mathrm{C}(\%)$ & $95 \mathrm{C}(\%)$ \\
$0 \mathrm{~m}$ (top) & 0 & 1.5 & 24.5 \\
$0.5 \mathrm{~m}$ & 4 & 2.7 & 74.0 \\
$1 \mathrm{~m}$ & 8 & 7.5 & 88.2 \\
$2 \mathrm{~m}$ & 16 & 70.2 & 96.0 \\
$2.5 \mathrm{~m}$ & 23 & 79.4 & 96.9 \\
\hline
\end{tabular}

${ }^{1}$ Placement of the $\mathrm{CO}_{2}$ sensors measured from the top where the animals entered the gondola $(0 \mathrm{~m}) .{ }^{2}$ Time taken from the top where the animals entered the gondola. $\mathrm{T}=0$ is start descending the gondola. ${ }^{3}$ Pigs were exposed to either $80 \% \mathrm{CO}_{2}(80 \mathrm{C})$ or $95 \% \mathrm{CO}_{2}(95 \mathrm{C})$ measured at the bottom of the well. 


\section{Electroencephalogram}

One 95C pig was not equipped with EEG electrodes since this pig was too restless during application of the equipment. Baseline EEG activity was successfully recorded in all other pigs and was representative for conscious and awake animals: high frequency, low amplitude waves (Figure 2a). During the $\mathrm{CO}_{2}$ exposure phase, in nine animals the EEG signal was lost due to muscular contractions during the stunning procedure. Of the 38 remaining continuously recorded animals, 20 were exposed to $80 \mathrm{C}$ and $18-95 \mathrm{C}$. In one pig exposed to $80 \mathrm{C}$ and two pigs exposed to $95 \mathrm{C}$ time to unconsciousness could not, but time to an iso-electric EEG could, be determined due to muscular contractions. In two pigs exposed to $80 \mathrm{C}$, time to unconsciousness could, but time to an iso-electric EEG could not be determined due to muscular contractions. Time to loss of consciousness based on EEG activity was longer $(P<0.001)$ in 80C pigs ( $47 \pm 6 \mathrm{~s}$; range $39-61 \mathrm{~s})$ than in $95 \mathrm{C}$ pigs ( $33 \pm 7 \mathrm{~s}$; range $21-44 \mathrm{~s}$ ). Time to an iso-electric EEG did not differ $(P=0.39)$ between $80 \mathrm{C}$ pigs ( $75 \pm 23 \mathrm{~s}$; range $54-150 \mathrm{~s}$ ) and $95 \mathrm{C}$ pigs (64 $\pm 32 \mathrm{~s}$; range 36-132 s).

\section{Respiration}

A good respiratory waveform signal lasting the entire $\mathrm{CO}_{2}$ treatment was successfully recorded in 31 pigs. In the other seven pigs the respiratory waveform could not be detected due to muscular contractions. Of the successfully recorded pigs, 17 were exposed to $80 \mathrm{C}$ and 14 were exposed to 95C. Time to the first apnea, was longer $(P<0.001)$ in $80 \mathrm{C}$ pigs ( $71 \pm 14 \mathrm{~s}$; range $52-103 \mathrm{~s}$ ) than in $95 \mathrm{C}$ pigs ( $44 \pm 7 \mathrm{~s}$; range $33-56 \mathrm{~s}$ ). Time to respiratory arrest was longer $(P=0.001)$ in $80 \mathrm{C}$ pigs $(235 \pm 61 \mathrm{~s}$; range 151-337 s) than in $95 \mathrm{C}$ pigs (152 $\pm 39 \mathrm{~s}$; range $96-209 \mathrm{~s}$ ).

\section{Behaviour}

Table 3 shows the latency to first, duration of (mean \pm SD) and number of behaviours observed in pigs exposed to $80 \mathrm{C}$ and $95 \mathrm{C}$ for $346 \mathrm{~s}$ No difference in walking was observed between $80 \mathrm{C}$ and 95C pigs. Both sitting and lying occurred earlier $(P<0.001)$ in 95C pigs compared to $80 \mathrm{C}$ pigs and duration of standing was shorter $(P<0.001)$ in $95 \mathrm{C}$ pigs compared to $80 \mathrm{C}$ pigs. Latency to all event behaviours (sniffing, gasping, retreat attempt, lying, muscular contractions, and loss of posture $)$ was longer $(P<0.0001)$ in $80 \mathrm{C}$ pigs than 95C pigs.

The time between first and last occurrence of gasping and muscular contractions was also assessed. The time between first and last gasp was longer $(P<0.001)$ in $80 \mathrm{C}$ pigs (206 $\pm 77 \mathrm{~s})$ compared to 95C pigs $(111 \pm 26 \mathrm{~s})$. The number of gasps while conscious, however, did not differ between $80 \mathrm{C}$ pigs $(6 \pm 3)$ and $95 \mathrm{C}$ pigs $(5 \pm 3)$. The time between first and last muscular contraction did not differ between $80 \mathrm{C}$ pigs ( $47 \pm 40 \mathrm{~s}$ ) and $95 \mathrm{C}$ pigs (59 $\pm 55 \mathrm{~s}$ ). 
TABLE 3

Latency to first, duration of (mean \pm SD) and number of behaviours observed in pigs exposed to $80 \%$ $\mathrm{CO}_{2}$ and $95 \% \mathrm{CO}_{2}$ for $346 \mathrm{~s}$.

\begin{tabular}{|c|c|c|c|c|}
\hline \multirow[b]{2}{*}{ Behaviour $^{1}$} & \multicolumn{2}{|c|}{$80 \% \mathrm{CO}_{2}$} & \multicolumn{2}{|c|}{$95 \% \mathrm{CO}_{2}$} \\
\hline & $\mathrm{N}^{2}$ & Mean \pm SD & $\mathbf{N}$ & Mean \pm SD \\
\hline \multicolumn{5}{|l|}{ States } \\
\hline Duration of standing (s) & $24 / 24$ & $31 \pm 6^{\mathrm{a}}$ & $24 / 24$ & $15 \pm 4^{\mathrm{b}}$ \\
\hline Latency to first walking (s) & $12 / 24$ & $6 \pm 9$ & $14 / 24$ & $9 \pm 5$ \\
\hline Duration of walking (s) & $12 / 24$ & $5 \pm 3$ & $14 / 24$ & $2 \pm 1$ \\
\hline Latency to first sitting (s) & $12 / 24$ & $31 \pm 3^{\mathrm{a}}$ & $12 / 24$ & $14 \pm 5^{\mathrm{b}}$ \\
\hline Duration of sitting (s) & $12 / 24$ & $3 \pm 2$ & $12 / 24$ & $3 \pm 3$ \\
\hline Latency to first lying (s) & $24 / 24$ & $34 \pm 5^{\mathrm{a}}$ & $24 / 24$ & $17 \pm 3^{b}$ \\
\hline Duration of lying (s) & $24 / 24$ & $310 \pm 5^{a}$ & $24 / 24$ & $328 \pm 3^{b}$ \\
\hline \multicolumn{5}{|l|}{ Events } \\
\hline Sniffing (s) & $24 / 24$ & $18 \pm 3^{\mathrm{a}}$ & $24 / 24$ & $7 \pm 2^{b}$ \\
\hline Latency to first retreat attempt (s) & $22 / 24$ & $22 \pm 6^{a}$ & $20 / 24$ & $10 \pm 4^{\mathrm{b}}$ \\
\hline Number of retreat attempts & $22 / 24$ & $2 \pm 1$ & $20 / 24$ & $2 \pm 1$ \\
\hline Latency to first gasp (s) & $24 / 24$ & $23 \pm 4^{\mathrm{a}}$ & $24 / 24$ & $9 \pm 3^{b}$ \\
\hline Number of gasps & $24 / 24$ & $30 \pm 9^{a}$ & $24 / 24$ & $14 \pm 3^{\mathrm{b}}$ \\
\hline Latency to first lateral head movement (s) & $8 / 24$ & $24 \pm 10$ & $12 / 24$ & $14 \pm 6$ \\
\hline Number of lateral head movements & $8 / 24$ & $2 \pm 1$ & $12 / 24$ & $1 \pm 1$ \\
\hline Latency to first jump (s) & $12 / 24$ & $34 \pm 5^{\mathrm{a}}$ & $11 / 24$ & $14 \pm 2^{b}$ \\
\hline Number of jumps & $12 / 24$ & $1 \pm 1$ & $11 / 24$ & $2 \pm 2$ \\
\hline Latency to first muscular contraction (s) & $21 / 24$ & $36 \pm 4^{\mathrm{a}}$ & $24 / 24$ & $20 \pm 6^{b}$ \\
\hline Number of muscular contractions & $21 / 24$ & $4 \pm 2$ & $24 / 24$ & $3 \pm 2$ \\
\hline Loss of posture (s) & $24 / 24$ & $44 \pm 5^{\mathrm{a}}$ & $24 / 24$ & $26 \pm 5^{b}$ \\
\hline Number of escape attempts & $0 / 24$ & - & $0 / 24$ & - \\
\hline
\end{tabular}

${ }^{1}$ First behaviour when entering the gondola was always standing.

${ }^{2}$ Number of pigs showing the specific behaviour.

$\mathrm{T}=0$ is start descending of the gondola into the well. Means with different letters are significantly different $(P<0.05)$.

Immediately after the end of the exposure, none of the 95C pigs showed a corneal reflex or breathing. In two $80 \mathrm{C}$ pigs, in two different runs, gasping was observed post stunning and these pigs were immediately immersed in $\mathrm{CO}_{2}$ for another 5 minutes. No corneal reflex was observed in these pigs. Concentrations of $\mathrm{CO}_{2}$ during these two runs were 79.2 and $82.4 \% \mathrm{CO}_{2}$.

\section{Behaviour in relation to the EEG}

Figure 3 shows the average latencies to the different behaviours and EEG-based loss of consciousness expressed by $80 \mathrm{C}$ (Figure 3a) or 95C (Figure 3b) pigs. Both $80 \mathrm{C}$ and $95 \mathrm{C}$ pigs showed a similar sequence of behaviours when exposed to the $\mathrm{CO}_{2}$. 
Figure 4 presents the range of individual time points at which the different behaviours started in relation to onset of EEG-based unconsciousness, based on visual assessment of EEG recordings, in $80 \mathrm{C}$ pigs (Figure $4 \mathrm{a}$ ) and $95 \mathrm{C}$ pigs (Figure $4 \mathrm{~b}$ ). In both $80 \mathrm{C}$ and 95C pigs, sniffing, latency to first retreat attempt, gasping, jumping and lying occurred before EEG-based loss of consciousness was observed. Muscular contractions were observed in $88 \%$ and $95 \%$ of $80 \mathrm{C}$ and $95 \mathrm{C}$ pigs, respectively, before EEG-based loss of consciousness. Loss of posture was observed in $63 \%$ and $81 \%$ of $80 \mathrm{C}$ and $95 \mathrm{C}$ pigs, respectively, before EEG-based loss of consciousness. Latencies to behaviours relative to onset of unconsciousness did not differ between both treatments.

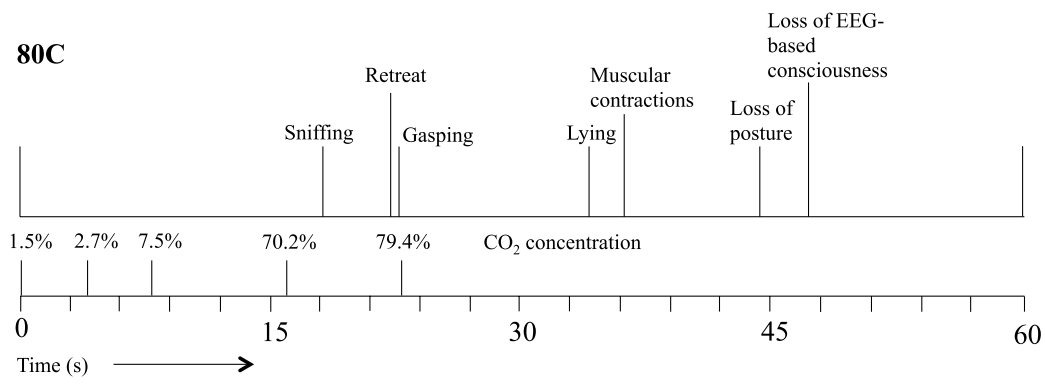

A

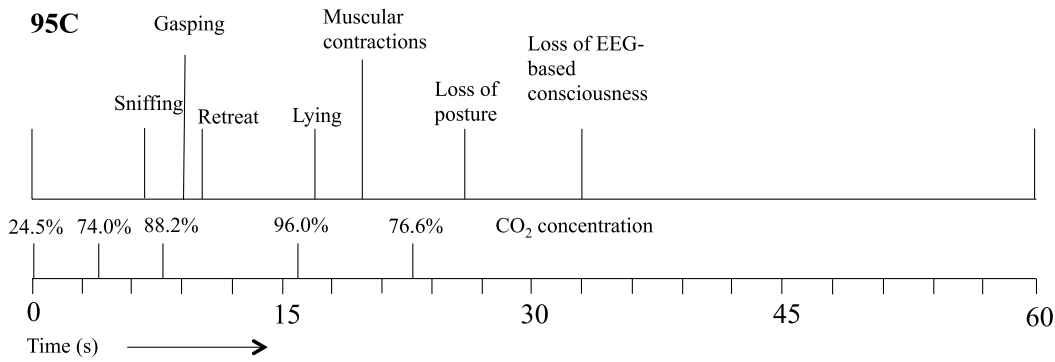

B

\section{FIGURE 3A,B}

Average latency (s) to the different behaviours expressed by pigs exposed to $80 \% \mathrm{CO}_{2}$ (80C, Figure 3a) or $95 \%$ $\mathrm{CO}_{2}$ (95C, Figure 3b). $\mathrm{T}=0$ indicates start descending of the gondola into the well pre-filled with $\mathrm{CO}_{2}$. The actual $\mathrm{CO}_{2}$ concentration was measured once at five different time points during descending of the gondola into the well. 


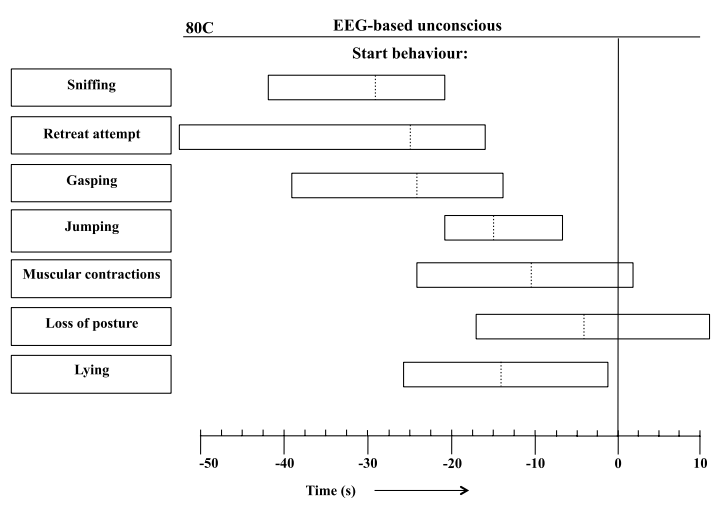

A

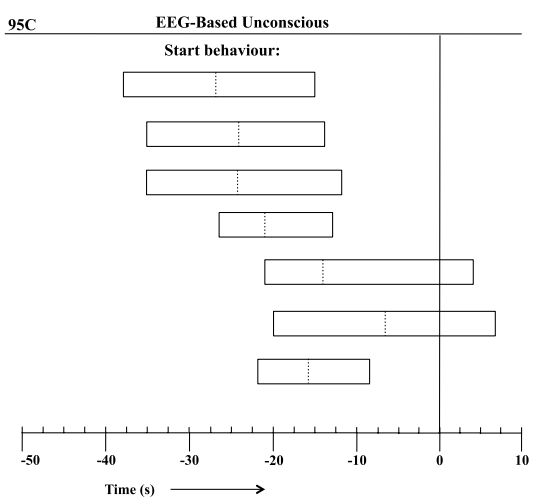

B

FIGURE 4A,B

Range of individual time points at which the different behaviours occurred in relation to EEG-based loss of unconscious ( $\mathrm{T}=0)$ during $\mathrm{CO}_{2}$ stunning in pigs exposed to $80 \% \mathrm{CO}_{2}(80 \mathrm{C}, n=24$, Figure $4 \mathrm{a})$ or $95 \% \mathrm{CO}_{2}(95 \mathrm{C}$, $n=24$, Figure $4 \mathrm{~b}$ ). Dotted lines indicate average values of all observations and left and right vertical lines of each block indicate minimum and maximum values for all observations.

\section{DISCUSSION}

According to EEG data and behavioural observations consciousness was reported to be lost during $\mathrm{CO}_{2}$ stunning ( $80-90 \%$ by volume in air) on average $30 \mathrm{~s}$ following onset of exposure based on both EEG data and behaviour (Raj and Gregory, 1996; EFSA, 2004). The time to loss of consciousness during $\mathrm{CO}_{2}$ stunning, however, remains debated (Tolo et al., 2010) and may depend on multiple factors, including the $\mathrm{CO}_{2}$ concentration applied and experimental design. An abrupt exposure to the gas mixture is known to induce a more rapid loss of consciousness in comparison with gradual immersion (Raj and Gregory, 1990). Some of the previous studies simulated commercial conditions (e.g. Rodriguez et al., 2008; Llonch et al., 2012) where pigs were immersed gradually to the required concentration at the bottom of a well. Part of the descending time was therefore taken up with transit through the interface between air and $\mathrm{CO}_{2}$ which is expected to prolong the conscious period. Based on EEG recordings, the pigs in the current study immersed in $80 \mathrm{C}$ and 95Clost consciousness, after on average 47 and $33 \mathrm{~s}$, respectively, after descending the gondola into the well. Our results are similar to those reported by Llonch et al. (2013) who reported loss of consciousness, based on EEG activity, $37.6 \mathrm{~s}$ after starting descending the gondola in a well pre-filled with $90 \% \mathrm{CO}_{2}$. Descending the gondola into the well also took $23 \mathrm{~s}$ in that experiment. During experiments performed by Forslid (1987) and Raj (1999), animals were immersed immediately (Forslid, 1987) 
or within 10 s (Raj, 1999) in a box that contained the modified atmosphere. Forslid (1987) concluded that low frequency activity dominated the EEG trace, indicative of unconsciousness, after 23-28 s in pigs immersed in 80C. Raj (Raj, 1999) observed loss of posture $17 \pm 3 \mathrm{~s}$ after pigs were exposed to the highest (80-90\%) $\mathrm{CO}_{2}$ concentration. Rodriguez et al. (2008), on the other hand, monitored brain activity using auditory evoked potentials and concluded time to loss of consciousness to be $60 \mathrm{~s}$ after being submersed into a well pre-filled with $90 \% \mathrm{CO}_{2}$. It should be noted, however, that the use of absence of evoked responses may provide more conservative times to loss of consciousness compared to loss of spontaneous EEG activity (Gregory and Wotton, 1986; Raj et al., 1991). Another possible explanation for the variety in time to loss of consciousness is that pigs differ in their responses to $\mathrm{CO}_{2}$ exposure and that these responses could be breed dependent, but also depend on the way in which animals are handled pre-slaughter (Grandin, 1992; Velarde et al., 2007; Terlouw et al., 2008).

During our study, time taken to loss of consciousness decreased with as $\mathrm{CO}_{2}$ concentration increased as previously reported by Raj and Gregory (1996) and EFSA (2004). Pigs subjected to $95 \mathrm{C}$ in the current study, were exposed to $74 \% \mathrm{CO}_{2}$ at $4 \mathrm{~s}$ after starting descending of the gondola, whereas pigs subjected to $80 \mathrm{C}$ were exposed to only $3 \% \mathrm{CO}_{2}$ at a similar depth. A faster and deeper respiration pattern observed in high $\mathrm{CO}_{2}$ concentrations results in an increased intake of $\mathrm{CO}_{2}$ and thereby increased efficiency of the stunning method, whereby the induction period is reduced and consequently time to loss of consciousness (Forslid, 1992).

Pigs exposed to $80 \mathrm{C}$ and 95C, revealed an iso-electric EEG after 75 and 64 s, respectively, indicating severely reduced central nervous system activity. Exposure to high $\mathrm{CO}_{2}$ concentrations is still reversible after 1-2 min (Forslid, 1987; Martoft et al., 2002), but prolonged exposure to $80 \% \mathrm{CO}_{2}$ resulted in death after approximately 2-3 minutes in the majority of the pigs in a study by Raj (1999). In the current study, 95C pigs had all died after the long exposure to $\mathrm{CO}_{2}$, confirmed by the absence of brain activity, breathing and brain stem reflexes. Two 80C pigs, however, displayed signs of gasping, although both animals showed an iso-electric EEG. Because recovery from $\mathrm{CO}_{2}$ stunning was not part of this study these pigs were immediately immersed for another 5 minutes in 80C. Since no corneal reflex after ascending of the gondola was observed in these pigs, it is likely that these gasps were only rudimentary brain stem activity and not signs of recovery from the $\mathrm{CO}_{2}$ exposure.

Brain activity, as presented in an EEG, is considered the most objective method available for the assessment of unconsciousness. This method, however, is only used for research objectives because its application holds numerous challenges during stunning and slaughter of livestock. One of the challenging aspects is that the EEG can be influenced by artefacts that are animal- or technical related (Teplan, 2002). Experimental controlled situations provide a better environment to limit these artefact sources than slaughter 
plants. Several studies on stunning and slaughter report disconnected electrodes or disrupted EEG activity in 9-71\% of the animals (Velarde et al., 2002; Gerritzen et al., 2004; Lambooij et al., 2006). The $\mathrm{CO}_{2}$ stunning procedure itself provides an additional challenge, as animals can move freely and (extensive) muscular contractions can easily disturb the EEG signal. Visual appraisal of EEG activity to assess the state of (un) consciousness has been applied during studies in poultry (McKeegan et al., 2013b), sheep (Verhoeven et al., 2015b) and veal calves (Verhoeven et al., 2016a). In addition to visual appraisal, EEG signals can be assessed using Fast Fourier Transformation (FFT). The output thereof represents the frequency composition of the signal, or alternatively formulated, how much power is presented in the different frequency bands. As this output is automatically derived, its results are considered more objective than visual appraisal. FFT analyses, however, necessitate a clean and stable EEG signal. Removal of artefacts is possible using certain types of filters, but this can also remove important information from the EEG trace as movement artefacts often occur in the $0-4 \mathrm{~Hz}$ range (Gerritzen, personal communication). Previous work by our group assessed the relation between onset of the different EEG stages, based on visual assessment of EEG activity, and spectral variables 'Total Power' and 'Spectral Edge Frequency', during propofol anaesthesia in sheep (Verhoeven et al., 2015b). There were strong correlations between onset of the different EEG stages based on visual EEG assessment and these two spectral variables ranging from 0.68 to 0.95 (Verhoeven, unpublished results). This supports the validity of visual assessment of EEG traces as conducted in the present study. It was not possible to perform continuous FFT analyses due to the muscular contractions of pigs that influenced the EEG traces.

Descending and ascending of the lift has been thought to induce fear in pigs. Holst (2002, cited by EFSA, 2004) found that $77 \%$ of the pigs stood motionless (freezing) in the gondola when lowered in atmospheric air (EFSA, 2004). The majority of these pigs started exploring the gondola while it was stationary. Based on these findings EFSA (2004) concluded that the vertical movement of the gondola itself induces fear in the pigs (EFSA, 2004). Dalmau et al. (2010) on the other hand, found that the time taken to cross the raceway and enter the gondola did not differ between subsequent training sessions and Velarde et al. (2007) found an increased percentage of pigs voluntarily entering the gondola in subsequent training sessions (Velarde et al., 2007; Dalmau et al., 2010). Subjective observations during air treatment days in the current study indicated no differences in behaviour of pigs entering the gondola on these days. The majority of pigs, however, stood motionless, in the gondola while descending and ascending. It cannot be excluded though, that the animals stood still to keep balance while moving up and down and not because animals were fearful (Bolhuis, personal communication). Though it is difficult to perceive what pigs experience during the $\mathrm{CO}_{2}$ induction period, the general opinion is that pigs respond aversively when exposed to high concentrations of $\mathrm{CO}_{2}$. 
Velarde et al. (2007) found increased times taken to cross the raceway and enter the gondola when pigs were exposed to a $\mathrm{CO}_{2}$ treatment compared to an air treatment and when exposed repeatedly to 70 or $90 \% \mathrm{CO}_{2}$. Therefore, exposure to $\mathrm{CO}_{2}$ was considered more aversive than exposure to atmospheric air. During the current study, there was a clear difference in pig behaviour when exposed to atmospheric air or $\mathrm{CO}_{2}$. None of the pigs were observed sitting or lying and no gasping, jumping, muscular contractions or escape attempts were observed on these 2 days. Exposure to $\mathrm{CO}_{2}$ stimulates respiration and pigs start to hyperventilate (Raj and Gregory, 1996). In humans, this is described as breathlessness which is known to increase with blood carbon dioxide levels (Stark et al., 1981). Moreover, $\mathrm{CO}_{2}$ is an acidic gas with a high solubility that together with water forms carbonic acid. With $\mathrm{CO}_{2}$ stunning, carbonic acid is formed when the $\mathrm{CO}_{2}$ dissolves in water from mucous membranes. It is therefore believed that $\mathrm{CO}_{2}$ causes irritation and pain in the lining of the nasal cavity when inhaled (Hari et al., 1997, Williams, 2004). There is continuing debate on which $\mathrm{CO}_{2}$ concentrations are most aversive to pigs since their behavioural responses vary with different $\mathrm{CO}_{2}$ concentrations. Signs of aversive behaviours include lateral head movements, retreat and escape attempts (Dodman, 1976; Raj and Gregory 1995 and 1996; Rodriguez et al., 2008). In a study by Rodriguez et al. (2008) lateral head movements were the first behaviour of pigs, on average, $10 \mathrm{~s}$ after initial exposure to $90 \% \mathrm{CO}_{2}$. Hartung et al. (2002) stated that the head movements were a clear indication that the animal had detected the gas and responded aversively to it. When confronted with an unpleasant situation, the response of a pig is often to back away (retreat) or escape (Dodman, 1976). Dodman (1976) observed that all pigs showed retreat attempts in $50-55 \% \mathrm{CO}_{2}$ and $37 \%$ of the pigs showed this response in $76-80 \% \mathrm{CO}_{2}$. In the current study, 92 and $83 \%$ of the pigs exposed to $80 \mathrm{C}$ and $95 \mathrm{C}$, respectively, showed at least one retreat attempt. Although the analgesic effect of $\mathrm{CO}_{2}$ has been demonstrated for higher concentrations (EFSA, 2004), the initial acute exposure to high carbon dioxide levels may induce an aversive response. This latter transient effect has been attributed to the irritating, and potential painful, influence on the mucous membranes. In the current study, no differences in the percentage of pigs showing retreat attempts could be determined between pigs exposed to 80C and 95C In two studies by Raj and Gregory none of the pigs showed escape attempts when exposed to $\mathrm{CO}_{2}$ concentrations lower than $30 \%$ or higher than $80 \%$ (Raj and Gregory, 1995 and 1996). The majority of pigs in a study by Velarde (2007), however, attempted to escape when exposed to $90 \% \mathrm{CO}_{2}$. During the current study, no escape attempts were observed. It is possible, however, that escape attempts were difficult to observe and this behaviour is in the current study marked as jumping or muscular contractions.

Gasping has not been considered an aversive behaviour as it occurs due to residual medullary activity in the brainstem when it becomes hypercapnic (Gregory, 2008a). It is a physiological reaction associated with breathlessness during the inhalation of high 
concentrations of $\mathrm{CO}_{2}$. All of the $80 \mathrm{C}$ and $95 \mathrm{C}$ pigs showed gasping before loss of consciousness, but the latency to gasping was shorter in 95C pigs than in $80 \mathrm{C}$ pigs. Duration from latency to gasping and loss of consciousness and the number of gasps while conscious, however, were similar in both groups. Figure 3 shows that gasping occurred closely in time with retreat attempts. It may be assumed that gasping does compromise animal welfare in conscious pigs, because it is associated with a sense of breathlessness (Beausoleil and Mellor, 2015).

Muscular contractions are observed in the majority of pigs exposed to high $\mathrm{CO}_{2}$ concentrations and it has been heavily debated whether they occur before or after animals have lost consciousness. It has been suggested that muscular excitations are the result by the lack of modulation of the caudal reticular formation from higher centers, particularly the cerebral cortex and physical activity during $\mathrm{CO}_{2}$ exposure might be an aversive response to the rostral reticular formation (EFSA, 2004). Zeller et al. (1987) and Rodriguez et al. (2008) stated that the respiratory distress induced by inhalation of gas. Dalmau et al. (2010) observed that time taken to cross the raceway and enter the gondola was lower in pigs without muscular excitations in the previous sessions than pigs with a high intensity of these muscle excitations, supporting the hypothesis that muscular excitations induce traumatism and pain. In that same study one pig was replaced in the first trial due to lameness. From these results, it was concluded that pigs might have associated the pain after or during the muscular excitation phase with the stunning system and consequently refused to enter the gondola in the following session. In this study, muscular contractions were observed in the majority of the pigs before they were considered unconscious and possibly compromising animal welfare. Since pigs were only exposed to the $\mathrm{CO}_{2}$ once, it was not possible to observe their response to the stunning system a second time.

Loss of posture has been suggested as first indicator of onset of unconsciousness (Raj and Gregory, 1996). Raj and Gregory (1996) defined it as the time to loss of posture (a recumbent state), whereas in the present study it was defined as "the pig is in a recumbent position with no sign of control of posture". The latter would indicate that a pig in the current study that would lay on the floor, but lifts it head up or still look up or around would not be considered having loss of posture and would be considered lying. Loss of posture was on average observed $10 \mathrm{~s}$ before loss of consciousness. When taking lying as a first indicator of onset of unconsciousness it was on average scored $15 \mathrm{~s}$ before loss of consciousness. Lying, however, was easier to score than loss of posture. None of the behaviours scored were able to exactly pinpoint time to loss of consciousness, but the loss of posture was on average closest and considered the first indicator of onset of unconsciousness.

The most important issue for welfare is what an animal experiences while conscious. The present study indicates that pigs respond aversively by means of lateral head movements, retreat attempts and possible jumping when exposed to $80 \mathrm{C}$ and 95C. Muscular 
contractions were observed in conscious pigs exposed to either $80 \mathrm{C}$ and $95 \mathrm{C}$ and this may compromise animal welfare. The number of behaviours and time from first occurrence of a behaviour relative to loss of consciousness, however, did not differ between the pigs exposed to $80 \mathrm{C}$ and $95 \mathrm{C}$ in the present study. The findings provide little reason to conclude on a behavioural basis that these atmospheres are greatly different in their impact on pig welfare.

\section{ACKNOWLEDGEMENTS}

We would like to thank the employees of the Institut de Recerca i Tecnologia Agroalimentaries (IRTA, Spain) for supplying the animals and use of their facilities and especially Joaquim Pallisera Lloveras and Xenia Moles Casselles for their support while collecting the data. We are grateful to Jan Peuscher for his technical assistance during the study and Danny de Koning for his advice on the statistical analyses. Vincent Hindle is acknowledged for his careful reading of the draft versions of this manuscript. Funding for this project was provided by the Dutch Ministry of Economic Affairs (KB-12-006.01002).

\section{AUTHOR CONTRIBUTION STATEMENT}

MV was involved in all steps leading to this manuscript and was responsible for the practical part of the study. AV contributed to the preparation of the practical part of the study. MG, AV, LH and BK contributed significantly to the discussion of the subject, and the development, writing, and final version of this paper.

\section{CONFLICT OF INTEREST STATEMENT}

The authors declare that the research was conducted in the absence of any commercial or financial relationships that could be construed as a potential conflict of interest. 
$\mathrm{CO}_{2}$ STUNNING IN PIGS | 103 
Publication

M.T.W. Verhoeven ${ }^{1,2}$, F.L.B. Meijboom ${ }^{3}$ M.A. Gerritzen ${ }^{1}$, L.J. Hellebrekers ${ }^{4}$, and B. Kemp ${ }^{2}$

${ }^{1}$ Wageningen University and Research Centre, Livestock Research, P.O. Box 338, 6700 AH Wageningen, The Netherlands

${ }^{2}$ Adaptation Physiology Group, Department of Animal Sciences, Wageningen University, P.O. Box 338, 6700 AH Wageningen, The Netherlands

${ }^{3}$ Ethiek Instituut, Utrecht University, Janskerkhof 13, 3512 BL Utrecht, The Netherlands

${ }^{4}$ Wageningen University and Research Centre, Central Veterinary Institute, P.O. Box 338, 8200 Lelystad, The Netherlands 


\section{SCIENTIFIC CONTRIBUTIONS TO \\ THE ETHICAL DEBATE ON STUNNING AND SLAUGHTER OF LIVESTOCK}

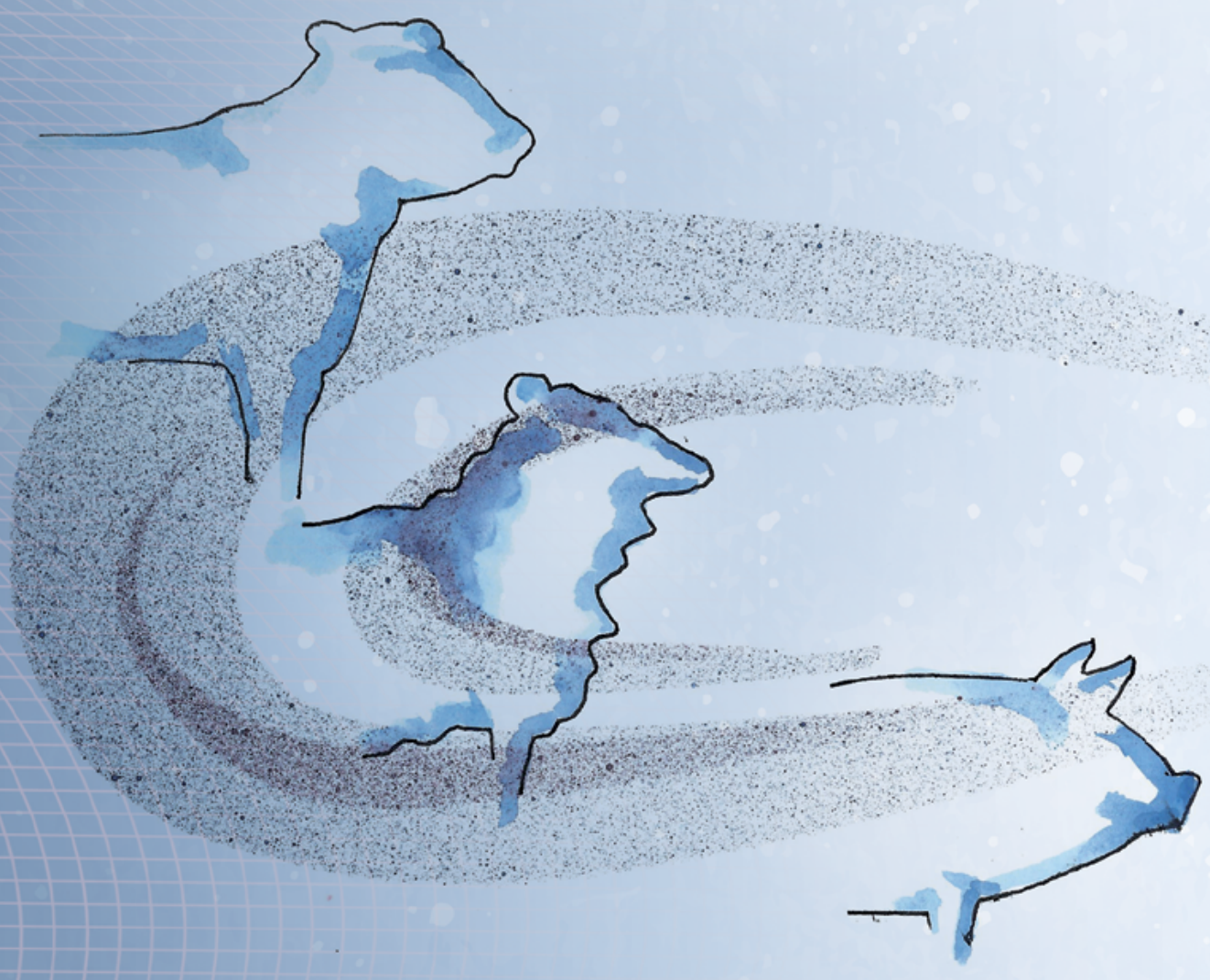




\section{ABSTRACT}

Slaughter of livestock not only requires technical competences, but the process is also guided by many complex questions and concerns of which some have a clear ethical component. The way in which we view animals has changed dramatically over time and has resulted in far-reaching and comprehensive legislation that regulates the treatment of animals on farm, during transport and at the slaughter plant. But even with legislation in place to safeguard animal welfare at slaughter, there still remains a plurality in views on the moral status of animals and the acceptability of the slaughter practice. The plurality in views is often observed in debates regarding these topics and understanding the origin of this plurality in views can improve the quality of such debates. Ethical reflection within, and empowerment of, parties in the sector are therefore needed. New scientific research may influence someone's view on the ethical acceptability of the slaughter practice. It is therefore important for all parties involved to be aware of the recent scientific developments regarding such topics. The aim of this paper is threefold. First, we analyse more in detail the plurality in moral views with regard to the slaughter process. Second, we present recent developments in scientific research on the stunning of animals. Third, we explore how this scientific research can contribute in the ethical debate on the acceptability of the current slaughter process based on the different moral views.

\section{INTRODUCTION}

Slaughter of livestock not only requires technical competences, but the process is also shaped by societal acceptance which in itself has a clear ethical component. There is a plurality in views that give rise to questions and concerns regarding the slaughter process. Part of these questions and concerns debate the use of animals to provide for meat products, while others deal with how the act of slaughter should be performed. These discussions are guided by different public values like: economic profit, public health, occupational safety, food quality and safety, and concerns for animal welfare (RDA, 2015). Such discussions are also guided by the relative importance that an individual or society assigns to these values. Public values will continue to shape the debate on what is acceptable and what is not, with regards to the slaughter of animals.

Public values and their relative weight change over time. In the last century especially the moral evaluation of animals changed dramatically (for most species at least). In the course of the twentieth century the more instrumental attitude most people held towards animals shifted to a more inclusive attitude. This inclusive attitude is characterised by the notion that there is no clear moral watershed between humans and animals. This resulted, on the one hand, in a growing resistance against the opinion that animals only 
exist to serve human purposes. On the other hand, it leads to the belief that animal life should be protected for the animal's own sake (van Zanden and Koolmees, 2003).

This attitude is reflected in many ways. Ruth Harrison's book 'Animal Machines' in 1964 was one of the first examples that puts this position forward in a clear and public way. She reported a number of practices of poor welfare, including confinement of the animals, social isolation, high stocking densities, the inability to show natural behaviour, and stress during transport (Harfeld, 2010). This book was not only one of the first to highlight these problems and the moral attitude towards animals that underlie it, but the issues addressed were subsequently also recognized in public debate. As a response to the public concern that arose following the release of her book, the UK government requested the Brambell Committee to 'Examine the conditions in which livestock are kept under systems of intensive husbandry and to advise whether standards ought to be set in the interests of their welfare, and if so what they should be' (Brambell, 1965). The conclusions and recommendations from the report of the Brambell Committee and the accompanying ethical considerations led to the development of specific national animal welfare legislation and also inspired legislation in many countries. Animal welfare legislation thereafter evolved further in accordance with an increase in knowledge, expertise and expectations from society related to farm animal welfare. EU member states now have adopted increasingly far-reaching and comprehensive legislation that regulates the treatment of animals on farm, during transport and at the slaughter plant. This legislation imposes a responsibility for those who handle livestock. Further, in 2009 the European Union (EU) member states ratified the Treaty of Lisbon, which acknowledges the recognition of the moral standing of animals in being '... sentient beings that are capable of feeling both pleasure and pain'. This acknowledgement paved the way for the development of the 'EU Animal Welfare strategy 2012-2015', designed to further improve welfare standards for farm-, zoo- and laboratory animals and ensure that these standards are applied in all EU member states. Nowadays, almost all (94\%) EU citizens, when asked, indicate that they find it important to protect the welfare of farmed animals and also that they feel that their welfare should be better protected than it is now (Eurobarometer, 2015).

Despite the increased attention for animal welfare and a comprehensive legal framework, there still remains a fundamental plurality in views on the moral status of animals and our duties towards animals. This diversity ranges from a view that principally objects against any form of rearing animals for human use, to the idea there is not any moral harm in using animals. This is not just the result of differences in opinion, but reflects a more fundamental disagreement with regards to the moral status and significance of animals. This disparity has its origin in diversity at the level of the interpretation of both facts and values. This context of pluralism directly influences the current debate on slaughtering animals in two ways: 
A) Despite a certain level of consensus about the moral importance of animal welfare, there still is disagreement on three related levels. First about whether animals are morally relevant, second on whether killing animals is morally problematic and third and finally there is plurality with regard to the question how we should weigh animalrelated values (e.g. animal welfare or animal health) to other ethical values (e.g. public health or occupational safety).

B) There is an ongoing scientific debate about how attention to animal welfare is best incorporated in the slaughter process.

The two ways described above, in which the plurality in views influences the current debate on slaughtering animals also influence each other. On the one hand, the relevance of a science-based solution to issues in the slaughter process depends on one's ethical position. For instance, if one considers an animal as a mere means to human ends, highly sophisticated measures to assess whether an animal is unconscious following the stun before bleeding will be considered superfluous. If, however, one acknowledges an animal as a sentient being that can suffer, this determination of unconsciousness appears to be essential for each type of slaughtering. On the other hand, new scientific information can influence one's ethical position. Knowledge derived from scientific research may well provide facts that are morally relevant. For instance, scientific research that leads to the development of a new stunning method that guarantees an immediate loss of consciousness may influence someone's view on the ethical acceptability of the slaughter practice. This interplay between science and ethics fits with the view on ethics as a dynamic process of reflection that takes facts, principles and intuitions into account and subsequently strives for achieving a reflective equilibrium (Heeger et al., 1998).

The aim of this paper is threefold. First, we analyse more in detail the plurality in moral views with regard to the slaughter process. Second, we present recent developments in scientific research on the stunning of animals. Third, we explore how this scientific research can contribute in the ethical debate on the acceptability of the current slaughter process based on the different moral views.

\section{THE PLURALITY IN VIEWS ON MORAL STATUS, SLAUGHTER OF ANIMALS AND BALANCING CONFLICTING VALUES}

As stated previously, there exists a plurality in moral views with regard to the slaughter process. This plurality reflects on three related levels. First, on the moral status of animals i.e. whether animals are to be considered morally important or not. Second, on the act of slaughter i.e. whether one finds the slaughter practice ethically acceptable and finally, third, how conflicting animal-related values and other ethical values are weighed. 


\section{Moral status}

Most livestock is slaughtered for meat production, which despite being everyday practice in itself is not morally neutral. The plurality in viewpoints on moral status of animals and our duties towards animals becomes distinctly obvious when it relates to this aspect of livestock farming. The concept of moral status it used to specify those entities towards which we may be considered to have moral obligations, as well as what we take those obligations to be (Warren, 1997). To define 'who' has moral status and what counts as constitutive elements for moral status is subject of a longstanding discussion. In Western philosophy there is a strong tradition to consider only humans to be moral subjects. For instance, some take the capacity to draft and enter contracts as a minimum condition to grant someone moral status. According to this contractarian view, animals lack moral status as they lack the capacity to build or be active part of contracts. Even though one may argue that animals and humans cooperate and that there is thus some form of contract (Rollin 2004), the animal's position is only indirectly represented by the voice of humans. Further to this view that denies animals to have independent moral standing, Warren (1997) identifies three views that each focus on one property as the single condition to grant an entity moral status, being: life, sentience and personhood.

The notion that life is the only property necessary to grant moral status to an entity implies that all living organisms have equal moral status.

Sentience has been proposed as property to grant moral status to an entity, because sentient beings are capable of experiencing pain, and we recognize it as our moral obligation not to inflict pain upon them without good reason.

The German philosopher Immanuel Kant and American philosopher Tom Regan both hold the view that beings that conform to a certain definition of personhood have full and equal moral status. Kant claimed that personhood consists in rational moral agency, being an individual's ability to make moral judgments based on some notion of right and wrong and to be held accountable for these actions. Kant stated that moral agency is characteristic of humans alone and thus only human beings can be granted moral status. Tom Regan, claimed equal moral status for all subjects-of-a-life and extended the principle of dignity to animals. He also claimed that higher non-human animals have rights of which the most basic right is not to be treated as a means to someone else's end. The set of psychological, physiological, emotional or intellectual properties that we associate with personhood, thus, may differ amongst people.

Next to granting moral status based on any of the three properties life, sentience or personhood, the ability to have and enter relationships can be a reason to grant moral status to an entity based on its social and ecological relationships, i.e. its memberships and role within a social or biological community. The relational theorist may state that where a close relationship between a person and animals exists (e.g. a shepherd and flock) special ethical limits on the treatment of these animals apply (Sandøe and Christiansen, 2008). 
Taken together, five views on moral status have been introduced. Following the contractarian view, animals lack moral status. According to the life only view, all living beings have equal moral status. The sentience view claims that sentience is a plausible criterion of moral status. Personhood, or having higher cognitive capacities, has also been proposed as criterion of moral status. In the relational view, moral status is granted based on the relations of an animal to human beings.

\section{Slaughter of animals}

Whether slaughter of animals is to be considered morally problematic depends on the moral status granted to an animal. The contractarian view focusses on livestock as a mere means to an end, and only one's own long-term interests count. The slaughter practice is not a moral dilemma for people that adhere to this view.

When life itself is the property used to grant an entity moral status, this implies that it is our moral obligation not to kill an animal as the act ends an animal's life and conflicts with respecting all life.

For those who state that sentience is the principal property to grant moral status to an animal, the act of slaughter per se is not a moral problem. However, slaughter is often associated with pain and distress and thus by harming sentient beings, the act of killing consequently becomes a moral problem. In principle, it may thus be morally permissible to kill animals for meat when they had a good (pleasant) life and are slaughtered in a painless way (Sandøe and Christiansen, 2008). According to DeGrazia (1996) sentient beings have the capacity to recognize danger and respond to it with specific behavioural actions. These behavioural responses indicate that animals strive to avoid life-threatening events and demonstrate behaviour in line with their 'survival instincts. The act of slaughter takes away this opportunity and frustrates future experiences.

Kant states that only human beings should be treated as ends in themselves, and as such having dignity or intrinsic value. Animals have only relative value as mere means to an end he claimed. The act of slaughter is not a moral problem per se, but it should be noted that we are morally obliged not to be unduly harmful to animals. Not necessarily just as an obligation to the animals, but also because it may conflict with the moral attitude of other human beings. Tom Regan, who claimed equal moral status for all subjects-of-life, stated that when you wish to find out whether a form of animal use is morally justified, one needs to determine whether the animal's use is respectful and preserves the animal's dignity and whether the animal is treated as an end in itself. Seen from this animal rights point of view, slaughter of animals is using them as a means to human gratification, whereby it does not treat them respectfully as ends in themselves. Therefore, in this line of thought, slaughter of animals cannot be morally justified.

According to the relational view, killing animals for food is acceptable as long as the keeping and using of the animal is not in violation with its membership and role within a social or biological community (Sandøe and Christiansen, 2008). 
Taken together, the ethical acceptability of the slaughter practice broadly differs for the five different moral views. Slaughter of animals is never permitted following the life only or animal rights view. The other views permit slaughter of livestock, but strongly disagree to what extent and under which circumstances this practice is to be allowed.

\section{Conflicting values}

Even when a person has a clear opinion on the moral status of animals and may therefore have a clear perspective on the act of slaughter, conflicts may arise between self-interest and our duties to animals. Animal related values are considered important, but other public values, such as food quality and safety and economic benefits, also play an important role in the discussion about the slaughter process. These different values may conflict, and the way conflicting values are weighed depends on the ethical theory one follows. According to the utilitarian view, the morally right course of action in any situation is the one that maximizes overall utility. So, the interests with most weight should prevail, no matter whose interests they are (Russow, 1990).

Another important ethical theory that can be used when weighing conflicting values is deontology. Deontologists believe that morality is a matter of duty. We have moral duties to do things that are right to do and moral duties not to do things that are wrong to do. Whether something is right or wrong does not depend on its consequences, as is the case with utilitarianism. Rather, an action is right or wrong in itself (Russow, 1990). These two theories can help us in dealing with the moral conflict: the value of animal life versus our moral duty to produce food. Whether slaughter of livestock gives rise to moral conflicts and how to deal with these conflicts depends on the ethical theory applied.

What becomes clear from the different views on moral status and differences in what we believe we owe animals is that the variation between viewpoints results in a situation whereby no single viewpoint can be used as the baseline for a morally acceptable treatment of farm animals at slaughter. Current legislation permits present slaughter practice, but also provides legislation aimed at avoiding unnecessary suffering in animals. Scientific facts can help us determine whether unnecessary suffering is indeed avoided and may thus shape our view on the ethical acceptability of the slaughter process. This process of measuring and weighing animal welfare at slaughter is often triggered by an intuitive response, but clearly also involves normative aspects and values and facts. Values and principles that lead to a specific view on the moral status of animals and to the acceptability of slaughter have been stated above. It is up to science to provide solid facts with regards to animal welfare during slaughter that can be used to further balance intuition and values. The three elements, namely intuition, principles/values and facts, interact and need to be balanced (striving for a so-called Reflective Equilibrium) before reaching a critical moral judgement (Daniels, 1979). Such an evaluation can be done by everyone, including policymakers, consumers, citizens and slaughter plant employees. The process 
clearly shows that appropriate handling of issues concerning animal welfare during slaughter requires an interplay between science and ethics, were empirical research and normative ethical theories are incorporated. This integration may further help the discussion on the acceptability of slaughter practices.

\section{SCIENTIFIC CONTRIBUTIONS TO ETHICAL CONCERNS WITH REGARDS TO STUNNING AND SLAUGHTER OF LIVESTOCK}

To avoid unnecessary suffering in animals at slaughter, stunning is mandatory according to EU legislation as it renders sentient animals unconscious, thereby preventing it from experiencing pain or distress. Abattoir employees are required to assess and determine unconsciousness after stunning before further processing (neck-cut, shackling and hoisting) takes place. In animals subjected to methods of slaughter prescribed by religious rites, unconsciousness must be ascertained before release from restraint. For those who find stunning of livestock at slaughter important, no matter the underlying reason, some of the relevant questions to be addressed in order to determine to what extent animals may suffer and if so, whether it can be avoided include:

1. how long does it take for animals to lose consciousness following stunning or nonstunned slaughter?

2. is there a chance for animals to recover from stunning before or during bleeding?

3. what percentage of animals is successfully stunned with the different stunning methods?

4. can the stunning effectivity be accurately determined? (i.e. can we determine whether an animal is unconscious after the stun or during the bleeding phase).

\section{How long does it take for animals to lose consciousness following stunning or non-stunned slaughter?}

Both mechanical and electrical stunning induce unconsciousness instantly following an effective stun (Finnie et al., 2002; Prinz, 2009; Verhoeven et al., 2016a). Loss of consciousness is not instant during $\mathrm{CO}_{2}$ stunning and was observed 21-61 s after first exposure to the gas (Verhoeven et al., 2016b). The use of $\mathrm{CO}_{2}$ as a stunning method is often debated due to animal welfare concerns. During the initial phase of $\mathrm{CO}_{2}$ stunning pigs respond aversively to the gas, since $\mathrm{CO}_{2}$ causes irritation of nasal mucosal membranes and $\mathrm{CO}_{2}$ is a strong respiratory stimulator that induces a sense of breathlessness prior to loss of consciousness (Manning and Schwartzstein, 1995; Nowak et al., 2007; Verhoeven et al., 2016b). Consciousness is also not lost instantly in animals slaughtered without stunning. Sheep that are slaughtered without stunning lose consciousness 2-24 seconds after the neck cut (Gregory and Wotton, 1984; Devine et 
al., 1986; Verhoeven et al., 2015b). Consciousness in cattle is generally lost later than in sheep and ranges from $2 \mathrm{~s}$ up to over 5 minutes after the neck cut (Nangeroni and Kennett, 1963; Newhook and Blackmore, 1982; Daly et al., 1988; Gibson et al., 2015; Verhoeven et al., 2016a). There is debate about the extent to which slaughter of conscious, i.e. sensible and/or aware, animals causes them pain and distress. The general opinion is that slaughter by neck incision of conscious animals causes pain and that bleeding while conscious may distress the animal (Gregory, 2005a; Johnson et al., 2012; Johnson et al., 2015).

Taken together, loss of consciousness is instant following mechanical- and electrical stunning. The methods that induce a gradual loss of consciousness $\left(\mathrm{CO}_{2}\right.$ stunning and non-stunned slaughter) have both been associated with animal welfare concerns during the conscious period.

\section{Is there a chance for animals to recover from stunning before or during bleeding?}

The objective of stunning an animal is to render it unconscious so it will not experience pain until death is induced by bleeding or cardiac arrest. It is important to know the duration of unconsciousness following an effective stun in order to determine the optimal time between stunning and start of the bleeding phase (stun-to-stick interval).

Under commercial conditions, mechanically stunned animals should be bled within 30 s following stunning to avoid regain of consciousness while bleeding (Grandin and Committee, 2013). O'Connor et al. (2015) observed that unconsciousness lasts on average about $30 \mathrm{~s}$ in sheep following electrical stunning, but with a high variability between animals, stressing the need to bleed the animals quickly following the stun. Exposure to high $\mathrm{CO}_{2}$ concentrations is still reversible after 1-2 minutes (Forslid, 1987; Martoft et al., 2002), but prolonged (346 s) exposure to 80 and $95 \% \mathrm{CO}_{2}$ resulted in death in $98 \%$ of pigs in our study, confirmed by the absence of brain activity, breathing and brain stem reflexes (Verhoeven et al., 2016b). Raj (1999) advised a stun-to-stick interval of 25 to 45 sec following $\mathrm{CO}_{2}$ stunning, depending on the gas mixture and concentration.

Taken together, when animals have a possibility of recovering from the stun, bleeding should occur as quickly as possible to minimize the chance for recovery.

\section{What percentage of animals is successfully stunned with the different stunning methods?}

In practice, unconsciousness is not always induced after a first stun. Risk factors for stun failures include a too long stun-to-stick interval, incorrect placement of the stunning device, in-properly maintained equipment and technical skills and experience of the operator (Anil et al., 2000; von Wenzlawowicz et al., 2012; Atkinson et al., 2013). 
In the study where we mechanically stunned calves, all calves immediately lost consciousness following the stun (Verhoeven et al., 2016a). Under commercial conditions, however, it is not uncommon that cattle need a second stun following captive bolt stunning. Field observations showed that percentages of captive-bolt stunned cattle showing clinical signs indicative for a risk of re-awakening completely varied from 2.1 to $32 \%$ in different field studies (Gregory et al., 2007; Gouveia et al., 2009; von Wenzlawowicz et al., 2012). In electrically stunned pigs these percentages ranged from 9.5 to $15 \%$ (von Wenzlawowicz et al., 2012). In the study on $\mathrm{CO}_{2}$ stunning of pigs that we performed, stunning was successful in all pigs (Verhoeven et al., 2016b). Field observations, however, showed that percentages of inadequately $\mathrm{CO}_{2}$ stunned pigs ranged from 0.14 to 66\% (Velarde et al., 2000; Nowak et al., 2007; Dalmau et al., 2009; Hartmann et al., 2010; Parotat et al., 2016).

Taken together, there is not one specific stunning method most effective in inducing stunning effectiveness is highly dependent of many factors. Experimental conditions provide a better environment to limit risks for stun failures compared to commercial conditions. To minimize the risks for stun failures it is important to ensure equipment is well maintained, settings of the stunner are according to protocol and slaughter plant employees are educated well.

\section{Can the stunning effectivity be accurately determined?}

Verhoeven et al. (2015a) showed that there is a wide range of indicators available to assess unconsciousness in livestock after stunning and that applicability and reliability of the different indicators vary per stunning method. To find reliable indicators for unconsciousness at slaughter, Verhoeven et al. (2015b and 2016a,b) performed multiple studies during which different indicators were assessed. Absence of rhythmic breathing and the corneal reflex were reliable indicators for unconsciousness following $\mathrm{CO}_{2}$ stunning in pigs (Verhoeven et al., 2016b). In calves mechanically stunned (i.e. penetrating captive bolt) absence of the threat-, withdrawal-, corneal-, and eyelid reflex indicated unconsciousness (Verhoeven et al., 2016a). Reliable indicators for unconsciousness in animals slaughtered without stunning included the absence of rhythmic breathing (assessed in sheep) and the absence of the eyelid- or corneal reflex (assessed in sheep and calves). These indicators were, despite being reliable, considered conservative measures of unconsciousness because their cessation always took longer (up to $2 \mathrm{~min}$ ) than time to unconsciousness based on brain activity. Some of the assessed indicators, however, were not reliable in assessing unconsciousness. Absence of the threat- and withdrawal reflex, for instance, were not reliable for assessing unconsciousness in animals slaughtered without stunning (Verhoeven et al., 2015b; Verhoeven et al., 2016a). The findings also showed that indicators for unconsciousness cannot always be assessed. Loss of rhythmic breathing and loss of posture, both indicative for unconsciousness, cannot 
be observed when animals are tightly restraint (Verhoeven et al., 2016a).

Taken together, unconsciousness can reliably assessed using the absence of rhythmic breathing or using the absence of the eyelid-or corneal reflex. They are, however, conservative measures of unconsciousness.

\section{INTEGRATING NEW SCIENTIFIC FACTS INTO THE REFLECTIVE EQUILIBRIUM ON STUNNING AND SLAUGHTER OF LIVESTOCK}

New scientific facts can shape someone's view on the ethical acceptability of the slaughter practice. The answers (facts) given to the four questions above may influence the remaining two elements (intuition and values/principles), together contributing to the formulation of the reflective equilibrium. Below we address some of the consequences the provided information may have on the ethical acceptability of the slaughter practice. The answers to the first question state that the conscious period is prolonged during $\mathrm{CO}_{2}$ stunning or non-stunned slaughter. It was also stated that the prolonged conscious period is associated with specific animal welfare concerns. If one wishes to avoid unnecessary pain and distress in the animal, as stated in EU legislation, the conscious period should be maximally limited and animals should preferably be rendered unconscious instantly following the stun. From our own observations, mechanical stunning will provide such a situation and according to the literature, electrical stunning also induces unconsciousness instantly. These two stunning methods are thus to be preferred for incorporation in the slaughter process to avoid unnecessary suffering, pain and distress in animals.

The answers to the second question allow for the conclusion that recovery from the stun is possible with all stunning methods, but will be limited in methods that not only stun, but also kill the animal. Since regaining consciousness after stunning is associated with unnecessary suffering, incorporating methods that induce death in the slaughter process are to be considered in order to avoid unnecessary suffering, pain and distress in animals. The answers to the third question state that stunning effectiveness is dependent on many factors. In our studies, under controlled conditions, stunning effectiveness was higher than under commercial conditions. Such conditions are therefore preferred to avoid unnecessary suffering, pain and distress in animals.

From the answers to the fourth question it may be concluded that unconsciousness can be assessed as such in each individual animal, but that not all indicators are suitable for obtaining reliable information. Indicators that can definitely ascertain an animal to be unconscious would be preferred to incorporate in the slaughter process to avoid unnecessary suffering, pain and distress in animals. 


\section{CONFLICTING VALUES WHEN INTEGRATING THESE NEW FACTS}

The combined answers to the four questions show that there are ways to avoid unnecessary suffering, pain and distress in animals during the slaughter process. However, when applied, conflicts will arise between aspects such as self-interest and our duties to animals. As stated previously, animal welfare is uniformly considered important, but other public values, such as food quality and safety and economic benefits, also play an important role in determining the different aspects of the slaughter process. How conflicting values are weighed depends on an individual's personal views and whether one, for example, follows a deontological reasoning or utilitarian reasoning.

From the discussion presented above it may be concluded that when consciousness is gradually lost during $\mathrm{CO}_{2}$ stunning animals may experience pain and distress. To decrease the amount of pain and distress in these animals, a different gas compared to $\mathrm{CO}_{2}$ may be used that also induce unconsciousness gradually, but limits the aversive behaviour. An examples of such a gas is the inert gas Argon that reduces the level of Oxygen in air, and that does not cause the aversive response in pigs that $\mathrm{CO}_{2}$ does (Dalmau et al., 2010). A major factor resulting in a preference for $\mathrm{CO}_{2}$ stunning over stunning with Argon is that $\mathrm{CO}_{2}$ is a less expensive gas. Changing the stunning system from $\mathrm{CO}_{2}$ to Argon will thus eventually increase the consumer's price of the meat product. A utilitarian approach will support the view that a small increase in the price of meat will not significantly decrease human welfare and is preferred over $\mathrm{CO}_{2}$ stunning, since it may improve animal welfare in millions of animals. A deontologist will look at what is morally right to do, no matter the consequences. If it is our duty not to cause unnecessary suffering, pain or distress in animals at slaughter, $\mathrm{CO}_{2}$ stunning should be replaced for another stunning method. If it is the duty of the slaughter plant to produce high quality meat at an acceptable market price, $\mathrm{CO}_{2}$ stunning may prevail over other stunning methods.

Another example is the use of assessing unconsciousness during the slaughter process. Indicators of which the absence clearly stated unconsciousness were the absence of rhythmic breathing, the absence of the eyelid reflex and absence of the corneal reflex. If one wishes to guarantee unconsciousness at slaughter and thus ensure animal welfare, each animal showing one of these reflexes should be re-stunned instantly. This is, however, easier to say than to actually incorporate in the slaughter plant. Slaughter line speeds should be adjusted (decreased) in a way that each individual animal can be assessed adequately. Further, abattoir employees must be trained to assess unconsciousness properly. And finally, since the three indicators we just mentioned are lost long after unconsciousness sets in, a lot of animals probably need (re)stunning, even though they are already unconscious, based on brain activity, at the time of testing. The consequence of such additional tasks for a slaughter plant will again be that meat becomes more expensive for the consumer. As stated earlier, from a utilitarian view, a small increase in the price of meat will not significantly decrease 
human welfare. A small increase in stunning effectiveness percentage will, however, have a positive impact on the welfare of millions of animals and must be strived at.

Looking at it from a deontological approach, it is the duty of a slaughter plant to avoid ineffective stunning in all animals. If there are indicators available that can reliably assess unconsciousness, the use of those indicators must be incorporated, preferably during multiple stages of the slaughter process. Again, if it is the duty of the slaughter plant to produce high quality meat at an acceptable market price, not testing all animals or using less conservative measures of unconsciousness may prevail.

\section{CONCLUSION}

In conclusion, certain moral views (e.g. life only view and animals rights view) reject the practice of livestock slaughter completely. Other more commonly held views, permit slaughter of livestock, but only when certain requirements are met, such as avoiding unnecessary pain and distress. Stunning livestock may do so and in this paper different ways of inducing unconsciousness were evaluated. The combined results show that consciousness can be lost either instantly or gradually and that, when lost gradually, animal welfare may be impaired. The results also show that the risks for stun failures are higher under commercial conditions compared to experimental conditions. Risks for stun failures can be decreased by implementing protocols and certain control check points. One of those control check points can be the assessment of unconsciousness during different stages of the slaughter process. Absence of rhythmic breathing, corneal reflex- and eyelid reflex are valid indicators of unconsciousness following captive bolt stunning, $\mathrm{CO}_{2}$ stunning and non-stunned slaughter. These indicators were, however, quite conservative and therefore imply that many animals need (re)stunning under current commercial practices. Both assessing unconsciousness and (re)stunning of animals at slaughter may avoid unnecessary pain and distress in these animals, but will also potentially affect other public values such as occupational safety and economic profit. The decision one ultimately takes on how the slaughter process must be adjusted to avoid unnecessary pain and distress in all livestock animals depends also on the ethical values and viewpoints one applies and consequently how the different public values are weighed. 

GENERAL DISCUSSION

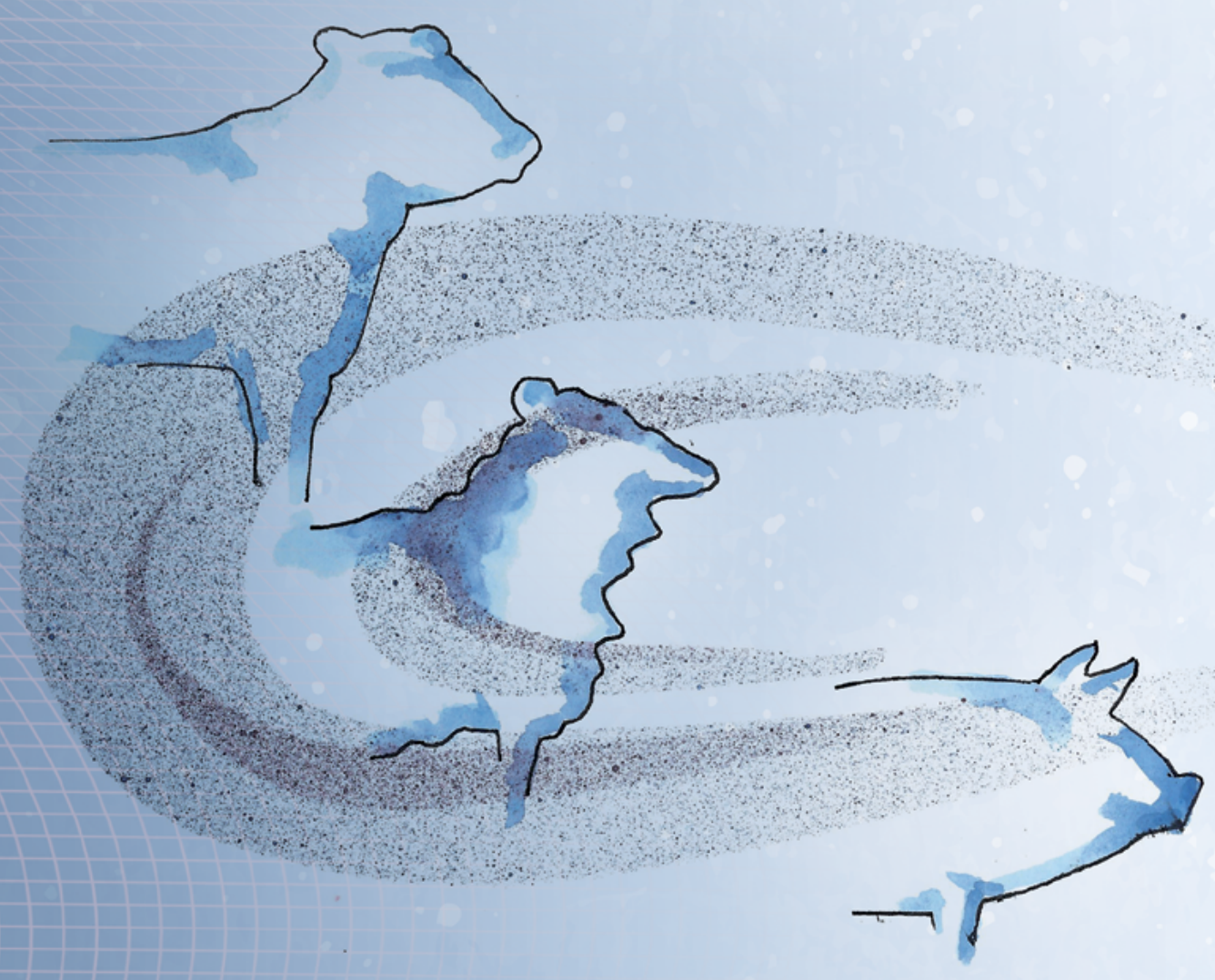




\section{INTRODUCTION}

Stunning animals to render them unconscious prior to bleeding is mandatory according to EU legislation since animals that are unconscious will not experience pain or distress (Johnson et al., 2012). Abattoir employees are therefore required to assess and confirm unconsciousness after stunning before further processing (neck-cut, shackling, hoisting and/or bleeding) takes place (Council Regulation (EC) 1009/2009).

The main methods for assessing unconsciousness focus on ascertaining the absence or presence of certain behaviours, physical signs and reflexes. Some of these indicators clearly indicate a conscious (e.g. vocalisations and coordinated walking movements) or unconscious state (e.g. absence of eye reflexes and absence of rhythmic breathing). Other indicators have a lower discriminating power (e.g. nystagmus, eyeball rotation and tongue protrusion) and are only used to provide complementary information (Grandin, 2002; Terlouw et al., 2016b). It is also important to note that under certain circumstances an indicator may be absent, though the animal is not unconscious. For example sheep often do not vocalise when injured, where a pig will scream loudly (Broom, 2001; EFSA, 2004). But the opposite, presence of an indicator while the animal is unconscious also occurs. Positive eye reflexes for instance may occur on the basis of residual brain stem activity, especially in electrically stunned animals (Roos and Koopmans, 1936; Blackmore and Delany, 1988; von Holleben et al., 2010). Under experimental conditions, the assessment of brain activity, as presented in an electroencephalogram (EEG), can be used to provide information on the state of consciousness and time to loss of consciousness (EFSA, 2013a; Verhoeven et al., 2015a; Terlouw et al., 2016b). Studies performed in an experimental set-up often assess either brain activity or behavioural indicators, physical signs and reflexes to determine loss of consciousness. Some studies (Rodriguez et al., 2008; Benson et al., 2012; McKeegan et al., 2013b; Sandercock et al., 2014) have assessed relationships between EEG activity and behavioural indicators, physical signs and reflexes, but interpretations of the results remain ambiguous. This is because species, stunning methods and assessed indicators differ between most studies. Better validated and applicable indicators are needed to reliably and reproducibly assess unconsciousness at slaughter. Knowledge derived from studies using EEG activity in combination with other indicators in experimental setups could lead to improvements regarding stunning methods and subsequently animal welfare at the slaughter plant.

The aim of this thesis was to study the times to loss of consciousness and relationships between behavioural indicators, physical signs, reflexes and brain activity, as presented in an EEG, in different species subjected to different stunning and slaughter methods. This allowed us to investigate the robustness of relationships between indicators and EEG activity under various circumstances. In this thesis, a single approach for all studies was 
used in the assessment of EEG traces and, where possible, similar behavioural indicators, physical signs and reflexes were assessed.

The general discussion is comprised of three main sections. In the first section, times to loss of consciousness, risks for stun failures and animal welfare concerns related to the most commonly used stunning methods are discussed. In the second section, different indicators and their relationships for the assessment of unconsciousness are evaluated. In the third and final section, practical implications of the work described in this thesis are discussed.

\section{TIMES TO LOSS OF CONSCIOUSNESS, RISKS FOR STUN FAILURES AND ANIMAL WELFARE CONCERNS IN DIFFERENT STUNNING AND SLAUGHTER METHODS}

A stunning method that, when applied correctly, induces unconsciousness instantly may be beneficial for animal welfare compared to the use of stunning and slaughter methods during which the conscious period is prolonged. In this section, the times it takes for animals to lose consciousness when subjected to different stunning and slaughter methods are evaluated. When stunning is not performed correctly, consciousness may be prolonged or regained in the animal and risk factors that can attribute to this are discussed for each method. At the end of each paragraph the different animal welfare concerns related to the specific stunning and slaughter method are described.

In the studies presented in this thesis, the times to loss of consciousness differed between stunning methods (Chapter 3,4,5). These differences occur because the stunning methods all induce unconsciousness in a different way (Terlouw et al., 2016a). Loss of consciousness can be instant following mechanical and electrical stunning, but can also be more gradual following $\mathrm{CO}_{2}$ stunning and slaughter without prior stunning (nonstunned slaughter). The first part of this section evaluates stunning methods that induce instant loss of consciousness following the stun. The second part of this section evaluates methods that induce a gradual loss of consciousness, including non-stunned slaughter.

\section{Direct loss of consciousness Mechanical stunning}

An example of a stunning method that, when performed correctly, induces unconsciousness instantly, is mechanical stunning, more specifically penetrating captive bolt stunning (Finnie et al., 2002). In the study described in Chapter 4, EEG activity was recorded in 24 penetrating captive bolt stunned veal calves and all but one calf displayed high-amplitude, slow-frequency waves immediately after stunning, indicating instant unconsciousness (Verhoeven et al., 2016a). Lambooy and Spanjaard (1981) and Zulkifli 
et al. (2014) found similar results, observing comparable high-amplitude, low-frequency EEG activity following the stun in all of their calves.

In the study described in Chapter 4, none of the calves were re-stunned. This indicates that when stunning conditions are appropriate, captive bolt stunning leads to immediate and sustained loss of consciousness. It is not uncommon, however, for cattle to be ineffectively stunned the first time. Studies performed under commercial conditions found that the percentage of captive bolt stunned cattle that had an increased risk of regaining consciousness or actually regained consciousness varied from 8.7 to $13.5 \%$ (Gregory et al., 2007; von Wenzlawowicz et al., 2012). Gouveia et al. (2009) did a field study ( $\mathrm{n}=2800)$ and observed signs of recovery in almost $32 \%$ of captive bolt stunned cattle.

Common causes of an ineffective stun include a lack of proper head fixation which makes it difficult to control location and orientation of the shooting (Bourguet et al., 2011, von Wenzlawowicz et al., 2012). But also age, breed and gender can increase the risk for stun failures (Gouveia et al., 2009). Stun failures were observed more frequently in bulls than in cows, steers and heifers because of the thickness of a bull's skull and the size of their head (Grandin, 2002; Gregory et al., 2007). In order for the captive bolt stunner to be effective in bulls, a higher bolt velocity is recommended $(>70 \mathrm{~m} / \mathrm{s})$ compared to the bolt velocity recommended for other cattle ( $>55 \mathrm{~m} / \mathrm{s}$ ) (Daly et al., 1987; Gregory and Grandin, 2007). Insufficient bolt velocity may be caused by poor maintenance of the stunner or a damp cartridge (Grandin, 2002). Moreover, technical skills and experience of the shooter play an important role in accurate stunning of cattle (Atkinson et al., 2013). Captive bolt stunning has not been associated with animal welfare concerns in literature, but one can understand that when consciousness is regained or re-stunning of the animal is needed, animal welfare is at risk.

Taken together, captive bolt stunning induces instant loss of consciousness when performed correctly. Risks for stun failures include lack of proper head fixation, age, breed and gender differences, poor maintenance of equipment and skills and experience of the shooter. The stunning method has not been associated with specific animal welfare concerns.

\section{Electrical stunning}

Electrical stunning is another stunning method that, when performed correctly, induces unconsciousness instantly. The stunning methodology is based on inducing epileptiform EEG activity associated with unconsciousness and is mostly applied in pigs and poultry in Europe (Raj and O'Callaghan, 2004; Raj et al., 2006). In New-Zealand, head-only electrical stunning, immediately followed by bleeding, is used on cattle as an alternative to non-stunned slaughter. The effectiveness of electrical stunning has been studied both under experimental- and commercial conditions. Velarde et al. (2002) studied loss of consciousness by assessing both readily observable indicators as well as EEG activity in 
lambs subjected to head-only electrical stunning. All animals were effectively stunned according to both EEG activity as well as based upon the absence of rhythmic breathing and absence of the corneal reflex.

As with captive bolt stunning, electrical stunning, however, is not always effective in inducing unconsciousness. Von Wenzlawowicz et al. (2012) assessed unconsciousness during 63 assessments in 6855 electrically stunned pigs and observed signs not compatible with sufficient depth of stunning in 3.3 to $10.9 \%$ of the pigs. In poultry slaughtered in the EU, electrical stunning is the most common method to render poultry unconscious. More specifically, electrical waterbath stunning is used to stun $81 \%$ of broilers in the EU (DG SANCO, 2012). The electrical parameters of a waterbath system (waveform, frequency, voltage and current) all differ between slaughter plants. This leads to variations in stunning effectiveness, where some setups cause cardiac arrest in birds, while others birds are able to recover (Wilkins et al., 1998). Also, the electrical impedance between individual birds differs which results in variation in current levels $(\mathrm{mA})$ encountered by the bird that may be lower than the predetermined current. As a result, consciousness may not be lost. It has been claimed that in commercial practice about one-third of birds is effectively stunned (Woolley et al., 1986). Prinz (2009) investigated the stunning effectiveness of different combinations of electrical waveforms and frequencies in broilers and observed stun effectiveness percentages of $>90 \%$, but also stun effectiveness percentages of $<50 \%$.

Risk factors for stun failures include incorrect placement of the electrodes (Sparrey and Wotton, 1997; Anil and McKinstry, 1998), insufficient current intensity or interruptions to the electrical current (Gregory, 2001) and regain of consciousness due to late or ineffective sticking (Anil et al., 2000).

If an electrical current is too low to induce unconsciousness, this may cause pain to the animal (Prinz, 2009). Another animal welfare concern relates to the use of the electrical waterbath stunning method in poultry. Hanging birds alive and conscious in an inverted position with their legs hanging in metal slots (also known as shackling) is a necessary act prior to the actual waterbath stunning. This shackling procedure in itself may be a painful procedure for the birds (Sparrey and Kettlewell, 1994; Gentle and Tilston, 2000). Another concern is that some birds may miss the stunner completely when they right their head and are therefore still conscious when their neck is cut or when reaching the scalding vat (Shields and Raj, 2010). In 2014, the EFSA Panel on Animal Health and Welfare has therefore recommended to develop and implement alternative stunning methods to the multiple bird electrical waterbath system to ensure adequate welfare (EFSA, 2014).

Taken together, electrical stunning induces instant loss of consciousness when performed correctly. Risk factors for stun failures include incorrect placement of the electrodes, insufficient current intensity or interruptions to the electrical current and late or 
ineffective sticking. Animal welfare concerns with this type of stunning are an insufficient current that only induces pain, but not unconsciousness. shackling birds prior to stunning and the possibility that birds may miss the stunner completely.

\section{Gradual loss of consciousness $\mathrm{CO}_{2}$ stunning}

In contrast to mechanical and electrical stunning, loss of consciousness is not instant during gas stunning. Different gasses or gas mixtures can be used to render animals unconscious based on anoxia (severely reduced levels of or complete lack of oxygen) hypercapnia (high levels of $\mathrm{CO}_{2}$ ) or a combination of hypercapnia and hypoxia (reduced oxygen levels). A commonly used gas stunning method for pigs and poultry in Europe is $\mathrm{CO}_{2}$ stunning.

The exact moment of onset of unconsciousness during $\mathrm{CO}_{2}$ stunning remains debatable, as with all stunning methods where consciousness is not lost at once (Tolo et al., 2010). The time to loss of consciousness depends on the $\mathrm{CO}_{2}$ concentration used and the speed at which animals are immersed into the highest concentration of $\mathrm{CO}_{2}$ at bottom of the well (Troeger, 1991; Raj and Gregory, 1996). Studies in pigs that examined brain activity, as presented in an EEG, reported loss of consciousness 12-60 s after initial exposure to $80-90 \%$ $\mathrm{CO}_{2}$ (Table 1). In the study described in Chapter 5, it was shown that pigs immersed in $80 \%$ and $95 \% \mathrm{CO}_{2}$ lost consciousness after on average $47 \pm 6 \mathrm{~s}$ (39-61 s) and $33 \pm 7 \mathrm{~s}(21-44$ s), respectively, after start descending the gondola into the well pre-filled with $\mathrm{CO}_{2}$. In the study described in Chapter 5, all pigs were effectively stunned. Studies performed under commercial conditions observed signs indicating ineffective stunning in 0.14 $66.3 \%$ of all pigs (Table 2). Differences, however, in the assessment of stunning effectiveness make comparisons between the studies difficult. A lot of variation was observed between the times at which the assessment took place following the stun, but also many different indicators were used to assess unconsciousness.

Risks for stun failures with this $\mathrm{CO}_{2}$ stunning include an insufficient exposure time to the gas or a too long time between end of stunning and start bleeding (also known as the stun-to-stick interval) (Velarde et al., 2000; Nowak et al., 2007)

Pigs exposed to increasing levels of $\mathrm{CO}_{2}$ display a range of behaviours before losing consciousness. Part of these behaviours are thought to reflect an aversive response to the gas. Signs of aversive behaviours include lateral head movements and retreat- and escape attempts (Dodman, 1976; Raj en Gregory 1995 and 1996). The aversive behaviours are considered a response to the $\mathrm{CO}_{2}$ that causes irritation of nasal mucosal membranes and the fact that $\mathrm{CO}_{2}$ is a strong respiratory stimulation that is known to induce a sense of breathlessness prior to loss of consciousness in humans (Manning and Schwartzstein, 1995, Raj, 2006). It may thus form a serious concern for animal welfare, as breathlessness is considered unpleasant for the animal (Beausoleil and Mellor, 2015). Barfod (1990) and 
TABLE 1

Time (mean $\pm \mathrm{SD}$, range (s)) to indicators for loss of consciousness in pigs immersed in different high concentrations $(\geq 80 \%)$ of carbon dioxide $\left(\mathrm{CO}_{2}\right)$

\begin{tabular}{|c|c|c|c|c|}
\hline Reference & $\mathrm{N}$ & $\begin{array}{l}\text { Indicator for loss of } \\
\text { consciousness }\end{array}$ & $\begin{array}{l}\text { Observed time (s) } \\
\text { mean } \pm \\
\mathrm{SD} / \text { range }\end{array}$ & $\begin{array}{l}\mathrm{CO}_{2} \\
\text { concentration } \\
(\%)\end{array}$ \\
\hline Dodman (1976) & 16 & Lateral recumbancy & $14 \pm 6(7-20)$ & $76-80$ \\
\hline Forslid (1987) & $6^{1}$ & Dominant delta $(<4 \mathrm{~Hz})$ activity & $21-30$ & $80^{2}$ \\
\hline Ring et al. (1988) & 44 & Changes in the EEG & $15-20$ & $80^{2}$ \\
\hline \multirow[t]{2}{*}{ Verhoeven et al. (2016b) } & 24 & Changes in the EEG & $47 \pm 6(39-61)$ & 80 \\
\hline & & Loss of posture & $44 \pm 5(36-54)$ & \\
\hline Holst (2002) & & Loss of posture & 22 & $85^{2}$ \\
\hline \multirow[t]{2}{*}{ Raj et al. (1997) } & 12 & Suppressed ECoG & $20 \pm 5(14-30 s)$ & $80-90$ \\
\hline & & Loss of SEPs ${ }^{3}$ & $21 \pm 7(17-25 s)$ & \\
\hline Raj (1999) & 12 & Loss of posture & $17 \pm 3$ & $80-90$ \\
\hline Forslid (1992) & $4^{1}$ & Dominant delta $(<4 \mathrm{~Hz})$ activity & $12 \pm 0.3$ & $90^{2}$ \\
\hline Raj and Gregory (1996) & 5 & Loss of posture & $15 \pm 3$ & 90 \\
\hline \multirow[t]{2}{*}{ Martoft et al. (2002) } & 6 & Burst suppression & 14 & 90 \\
\hline & & Loss of balance & $22 \pm 1$ & 90 \\
\hline Rodriguez et al. (2008) & 25 & Changes in MLAEP & $>60$ & 90 \\
\hline Llonch et al. (2013) & 15 & Loss of brain responsiveness & 43 & 90 \\
\hline \multirow[t]{2}{*}{ Verhoeven et al. (2016b) } & 24 & Changes in the EEG & $33 \pm 7(21-44)$ & 95 \\
\hline & & Loss of posture & $26 \pm 5(16-35)$ & \\
\hline
\end{tabular}

${ }^{1}$ All pigs tested twice. ${ }^{2}$ Cited by EFSA (2004). Welfare aspects of stunning and killing methods. Report EFSA-Q-2003-093 AHAW / 04-027, page 105. ${ }^{3}$ Somatosensory evoked potentials

Erhardt et al. (1989) on the other hand did not find conclusive evidence that $\mathrm{CO}_{2}$ irritates the membranes and concluded that the observed gasping is not a sign of respiratory distress, but merely a normal response to the excessive $\mathrm{CO}_{2}$ in the body. The general opinion, however, is that the initial phase of $\mathrm{CO}_{2}$ stunning is aversive to pigs (Nowak et al., 2007). Pigs that were stunned with 80 or $95 \% \mathrm{CO}_{2}$, as described in Chapter 5 , were in the days prior to the stunning habituated to the ascending and descending of the dip-lift containing atmospheric air. During the actual $\mathrm{CO}_{2}$ exposure, all pigs displayed aversive behaviours including lateral head movements, retreat attempts and jumping that were not observed when pigs were exposed to atmospheric air. Based on those observations, it is likely to assume that the stunning with $\mathrm{CO}_{2}$ was aversive to the pigs. The conscious period during which pigs can potentially experience pain and distress when stunned with $80 \% \mathrm{CO}_{2}$ (Chapter 5) was on average $25 \pm 9 \mathrm{~s}$ when calculating the time from first retreat attempt to EEG-based unconsciousness. In pigs exposed to $95 \% \mathrm{CO}_{2}$ this period was on average $24 \pm 6 \mathrm{~s}$. 
TABLE 2

Percentage of pigs showing signs indicative for ineffective $\mathrm{CO}_{2}$ stunning observed by different studies performed under commercial conditions

\begin{tabular}{|c|c|c|c|c|}
\hline Reference & $\mathbf{N}$ & $\begin{array}{l}\text { Indicator for ineffective } \\
\text { stunning }\end{array}$ & $\begin{array}{l}\text { Percentage of } \\
\text { ineffectively stunned } \\
\text { pigs }\end{array}$ & Time of assessment \\
\hline \multirow{3}{*}{$\begin{array}{l}\text { Velarde } \\
\text { et al. }(2000)\end{array}$} & 4.547 & Response to nose-prick & 3.7 and $42.8 \%$ & $30-35 \mathrm{~s}$ after stunning \\
\hline & & & & $\begin{array}{l}\text { Assessed in } 2 \text { different } \\
\text { abattoirs }\end{array}$ \\
\hline & & Righting reflex & 12.8 and $33.3 \%$ & \\
\hline \multirow[t]{3}{*}{$\begin{array}{l}\text { Nowak } \\
\text { et al. (2007) }\end{array}$} & 460 & $\begin{array}{l}\text { Corneal- and eyelid } \\
\text { reflex }\end{array}$ & $5.9-66.3 \%$ & 25-30 s after stunning \\
\hline & & & & $\begin{array}{l}6 \text { treatments with } \\
\text { different } \mathrm{CO}_{2} \\
\text { concentrations and } \\
\text { stun-to-stick intervals }\end{array}$ \\
\hline & & Nose prick reaction & $0-29.1 \%$ & \\
\hline \multirow{5}{*}{$\begin{array}{l}\text { Dalmau } \\
\text { et al. }(2009)\end{array}$} & 600 & Rhythmic breathing & $0-45 \%$ & Following stunning \\
\hline & & & & $\begin{array}{l}\text { Assessed in } 10 \text { slaughter } \\
\text { plants }\end{array}$ \\
\hline & & Righting reflex & $0-25 \%$ & \\
\hline & & Vocalisations & $0-7 \%$ & \\
\hline & & Corneal reflex & $0-60 \%$ & \\
\hline \multirow{2}{*}{$\begin{array}{l}\text { Hartmann } \\
\text { et al. }(2010)\end{array}$} & 2.650 & Corneal reflex & $6.2 \%$ & 69-101 s after stunning \\
\hline & 2.100 & Corneal reflex & $17.1 \%$ & 28-35 $\mathrm{s}$ after stunning \\
\hline
\end{tabular}

According to European legislation, the $\mathrm{CO}_{2}$ concentration used during $\mathrm{CO}_{2}$ stunning should at least be $80 \%$, but many slaughter plants use $90 \% \mathrm{CO}_{2}$ or higher in attempts to increase throughput at the slaughter plant (Velarde et al., 2007). When looking at these high concentrations of $\mathrm{CO}_{2}$, Nowak et al. (2007) observed higher lactate levels, indicative of stress, in pigs exposed to $80 \% \mathrm{CO}_{2}$ compared to pigs exposed to $90 \% \mathrm{CO}_{2}$. In pigs exposed to $80 \%$ or $95 \% \mathrm{CO}_{2}$, as described in Chapter 5 , the number of behaviours and time from first occurrence of a behaviour relative to loss of consciousness, however, did not differ. The findings from Chapter 5 provided evidence to conclude, based on behavioural characteristics, that these atmospheres ( 80 and $\left.95 \% \mathrm{CO}_{2}\right)$ are similar in their impact on pig welfare.

Taken together, loss of consciousness in pigs stunned with high levels of $\mathrm{CO}_{2}$ occurs between 12 and $60 \mathrm{~s}$ after initial exposure to the gas. Risks for stun failures include insufficient exposure times or a too long stun-to-stick interval. The stunning method has been associated with animal welfare concerns, mainly with the possibility of pigs experiencing pain and distress during the conscious period. 


\section{Non-stunned slaughter}

Slaughter without prior stunning is prohibited under EU legislation, with the exception of particular methods of slaughter prescribed by religious rites (Council Regulation (EC) 1099/2009). When slaughtering an animal that has not been stunned, bleeding will cause the loss of consciousness and eventually death. Approximatively 40 to $60 \%$ of total blood volume is lost during the bleeding phase (Warriss, 1984). Severe bleeding impairs oxygenation and delivering of nutrients to tissues and organs and produces a state of shock. The mental state of humans that lose over $30 \%$ of their blood volume, has been described as confused (30-40\% blood loss) or lethargic ( $>40 \%$ blood loss) (Gutierrez et al., 2004). The rapid effects of bleeding on brain function are linked to the brain's small storage capacity, and high needs for glucose and oxygen (Terlouw et al., 2016a).

Times to loss of consciousness in livestock slaughtered without prior stunning vary between species, but also between individuals of the same species. Following the results summarized in Table 3, sheep lose consciousness within 2 to 20 seconds after the neck cut. Results from the study described in Chapter 4 showed similar results where loss of consciousness in sheep was observed, based on EEG activity, $15 \pm 4 \mathrm{~s}$ after the neck cut. There is far less agreement on the time to loss of consciousness in non-stunned slaughtered cattle. Results from different studies (Table 4) show that there is considerably more variation in time to loss of consciousness in cattle compared to sheep. Some studies in cattle suggest a rapid loss of consciousness (4-10 s), based on EEG activity, following the neck cut (Nangeroni and Kennett, 1963; Bager et al., 1992). Other studies report EEG-based loss of consciousness, on average $66 \pm 21 \mathrm{~s}$ and $75 \pm 80 \mathrm{~s}$ after the neck cut, but the possibility of an intermittent return to consciousness for up to 123 to $323 \mathrm{~s}$ after the neck cut has also been reported (Newhook and Blackmore, 1982a; Blackmore et al., 1983). Following the results from different studies described in Table 4, cattle seem to lose consciousness somewhere between a couple of seconds up to five minutes after the neck cut. In the study described in Chapter 5, the time to loss of consciousness was on average $109 \pm 32 \mathrm{~s}$ (ranging from 43 to $140 \mathrm{~s}$ ) after the neck cut in calves that were slaughtered in an upright position. The time to loss of consciousness was on average 49 $\pm 25 \mathrm{~s}$ (ranging from 14 to $109 \mathrm{~s}$ ) after the neck cut in calves that were slaughtered in an inverted position.

Studies that have investigated times to loss of consciousness following non-stunned slaughter in poultry are scarce. The time to loss of consciousness in birds is thought to be intermediate between cattle and sheep (Terlouw et al., 2016a). In chickens $50 \%$ of the power of the electrical activity in the brain, indicative for loss of consciousness, was observed $19 \pm 4 \mathrm{~s}$ after both carotid arteries were cut (Gregory and Wotton, 1986). Barnett et al. (2007) concluded that broilers slaughtered without prior stunning lost consciousness between 12 and 15 seconds after the cut based on their loss of ability to remain a standing posture. One bird in that study was observed in a standing posture for up to 26 seconds. 
TABLE 3

Time post cut (mean \pm SD, range (s)) to indicators for loss of consciousness in sheep slaughtered without prior stunning.

\begin{tabular}{|c|c|c|c|}
\hline Reference & $\mathrm{N}$ & Indicator for loss of consciousness & $\begin{array}{l}\text { Observed time } \\
\text { post cut (s) } \\
\text { mean } \pm \text { SD } \\
\text { (range) }\end{array}$ \\
\hline Nangeroni and Kennet (1963) & 5 & Relevant EEG changes & $7 \pm 5(3-15)$ \\
\hline Schulze et al. (1978) & 17 & Relevant EEG changes & $4-6^{1}$ \\
\hline Newhook and Blackmore (1982b) & 5 & EEG amplitude $<10 \mu \mathrm{V}$ and $>35 \mu \mathrm{V}$ & $2-7$ \\
\hline Gregory and Wotton (1984) & 20 & $\begin{array}{l}\text { Time to loss of brain responsiveness } \\
\text { determined from } \mathrm{VEPs}^{2}\end{array}$ & $14 \pm 1$ \\
\hline \multirow[t]{2}{*}{ Blackmore (1984) } & $\begin{array}{l}3^{3} \\
2\end{array}$ & $\begin{array}{l}\text { Loss of ability to stand and loss of apparent } \\
\text { coordinated attempts to rise }\end{array}$ & $\begin{array}{l}3 \pm 1(2-3) \\
4 \pm 1(3-4)\end{array}$ \\
\hline & & Loss of apparent coordinated attempts to rise & $\begin{array}{l}10 \pm 1(9-11) \\
9 \pm 1(8-9)\end{array}$ \\
\hline Devine et al. (1986) & & EEG amplitude $<10 \mu \mathrm{V}$ & $14 \pm 7(8-22)$ \\
\hline Tidswell et al. $(1987)^{4}$ & $2^{3}$ & Decline of EEG to $<10 \mu \mathrm{V}$ & 7 and 8 \\
\hline \multirow[t]{2}{*}{ Kallweit et al. $(1989)^{5}$} & 4 & ECoG iso-electric & $13(5-20)$ \\
\hline & 9 & EcoG iso-electric & $12(8-17)$ \\
\hline Verhoeven et al. (2015b) & $21^{3}$ & Start of high amplitude, low frequency EEG & $15 \pm 4(6-24)$ \\
\hline
\end{tabular}

${ }^{1}$ Cited by von Holleben et al. (2010) who stated that the original report and data of the project Hazem et al. (1977) revealed that, though the authors concluded loss of consciousness being highly probable after 4 to 6 seconds in the publication, they recorded unchanged EEG until 8 seconds after the cut and concluded in the original report that sheep lost consciousness latest 10 seconds after the neck cut. ${ }^{2}$ Visual evoked potentials. ${ }^{3}$ Lambs. ${ }^{4}$ Observations following decapitation. ${ }^{5}$ Cited by von Holleben et al. (2010). Four sheep were non-stunned slaughtered according to halal rites and 9 sheep were non-stunned slaughtered according to shechita rites.

Terlouw et al. (2016a) observed loss of consciousness in chickens and turkeys after on average $35 \mathrm{~s}$, ranging in chickens from 12 to $202 \mathrm{~s}$ and in turkeys from 18-51 s (Bourguet, Deiss and Terlouw, personal observations, cited by Terlouw et al., 2016a).

Some of the variation between studies can be attributed to different indicators used to assess times to loss of consciousness. Indicators used in the different cited studies consisted of loss of standing posture (Gregory et al., 2010), onset of low frequency activity (Nangeroni and Kennett, 1963) loss of evoked potentials (Daly et al., 1988) or changes in EEG activity (Verhoeven et al., 2015b; Verhoeven et al., 2016a). But even within a single study, the variation between animals remains large. Gregory et al. (2010) observed an average time to collapse of $20 \mathrm{~s}$ after the neck cut in 174 cattle. In $8 \%$ of those cattle, however, it took $60 \mathrm{~s}$ or more to collapse after severing both carotid arteries.

Two phenomena are believed to contribute to prolonged consciousness in cattle during the bleeding phase. In a proportion of the slaughtered cattle, occlusion of the severed arteries, and therefore impaired bleeding out may occur. Gregory et al. (2006) observed this in 25 
TABLE 4

Time (mean \pm SD, range (s)) post cut to indicators for loss of consciousness in calves and adult cattle slaughtered without prior stunning.

\begin{tabular}{|c|c|c|c|}
\hline Reference & $\mathrm{N}$ & Indicator for loss of consciousness & $\begin{array}{l}\text { Observed time post } \\
\text { cut }(s) \\
\text { mean } \pm \text { SD (range) }\end{array}$ \\
\hline \multicolumn{4}{|l|}{ Calves } \\
\hline Nangeroni and Kennett (1963) & 5 & Relevant EEG changes & $6 \pm 1(4-7)$ \\
\hline Schulze et al. (1978) & 10 & Relevant EEG changes & $10 \mathrm{~s}$ (up to $18 \mathrm{~s}, 24 \mathrm{~s})^{1}$ \\
\hline $\begin{array}{l}\text { Newhook and Blackmore } \\
\text { (1982a) }\end{array}$ & 8 & $\begin{array}{l}\text { Low voltage, fast activity waves and } \\
\mathrm{EEG}<10 \mu \mathrm{V} \text { and }>35 \mu \mathrm{V}\end{array}$ & $66 \pm 21(34-85)^{2}$ \\
\hline Blackmore et al. (1983) & 3 & $\begin{array}{l}\text { Low voltage, fast activity waves and } \\
\text { EEG }<10 \mu \mathrm{V} \text { and }>35 \mu \mathrm{V}\end{array}$ & $75 \pm 80(28-168)^{3}$ \\
\hline Blackmore (1984) & 5 & & \\
\hline Devine et al. (1986) & 1 & $\mathrm{EEG}<10 \mu \mathrm{V}$ & 79 \\
\hline Bager et al. (1992) & 6 & Relevant ECoG changes & Within 10 \\
\hline Lambooij et al. (2012) & 31 & $\begin{array}{l}\text { Reduction of } 60 \% \text { in } \\
\text { Cordimanes scores (EEG) }\end{array}$ & 80 \\
\hline \multirow[t]{2}{*}{ Verhoeven et al. (2016a) ${ }^{4}$} & 7 & Relevant EEG changes & $109 \pm 32(43-140)$ \\
\hline & 25 & Relevant EEG changes & $49 \pm 25(17-109)$ \\
\hline \multicolumn{4}{|l|}{ Cattle } \\
\hline \multirow[t]{4}{*}{ Daly et al. (1988) } & 7 & Loss of $\mathrm{SER}^{5}$ & $77 \pm 32(32-126)$ \\
\hline & 8 & Loss of $\mathrm{VER}^{6}$ & $55 \pm 32(20-102)$ \\
\hline & 8 & $\mathrm{EEG}<10 \mu \mathrm{V}$ & $75 \pm 48(19-113)$ \\
\hline & 8 & $\begin{array}{l}\text { Onset of high amplitude } \\
\text { low frequency waves }\end{array}$ & $7.5 \pm 2(5-13 s)$ \\
\hline Gregory et al. (2010) & 174 & Final physical collapse & $20 \pm 33$ \\
\hline \multirow[t]{2}{*}{ Gibson et al. $(2015)^{7}$} & 561 & Final physical collapse & $14 \pm 1(1-257)$ \\
\hline & 83 & Final physical collapse & $19 \pm 1(4-86)$ \\
\hline
\end{tabular}

${ }^{1}$ Cited by von Holleben et al. (2010) who stated that the original report and data of the project Hazem et al. (1977) revealed that, though the authors concluded loss of consciousness being highly probable in calves after 10 seconds, they recorded unchanged EEG until 18 seconds after the cut and in one animal, which had to be re-cut because of obviously slow bleeding, the EEG showed only very small changes until 24 seconds after the first cut. ${ }^{2}$ Possible sensibility for up to $123-323 \mathrm{~s}$ after the neck cut. ${ }^{3}$ Possible sensibility for up to 130-187 s after the neck cut. ${ }^{4}$ Slaughtered in an upright position $(n=7)$ or inverted position $(n=25) .{ }^{5}$ Somatosensory evoked responses. ${ }^{6}$ Visual evoked responses. ${ }^{7}$ Cattle received a conventional low neck cut $(\mathrm{n}=561)$ or a high neck cut $(\mathrm{n}=83)$

and $16 \%$ of bobby calves and large cattle, respectively, slaughtered without prior stunning. Gregory et al. (2010) observed that $71 \%$ of cattle taking longer than 75 s to collapse, had a false aneurysm in the cardiac end of the carotid artery of at least $3 \mathrm{~cm}$ in diameter. Secondly, the vertebral artery in bovines stays intact when the neck is cut and continues to supply blood to the brain increasing the time to loss of consciousness (Daly et al., 1988). 
Further variation in times to loss of consciousness may depend on neck cutting procedures that can vary due to the size of the animal, the setting of the machine, or, in the case of manual methods, the skills and experience of the operator (Gregory and Wotton, 1986). Gregory et al. (2008) observed large differences in the number of cuts needed per animal between different slaughter facilities. These differences may partially be attributed to the technical skills of the operator. In the studies described in Chapter 4 and 5, one person performed the neck cut, in order to standardize the procedure as much as possible. None of the sheep in the study described in Chapter 4 needed a second cut. The study described in Chapter 5, however, took place at a commercial slaughter plant, were the slaughter man himself was empowered to decide when an additional cut was necessary. No clear criteria were defined for this. In total 15 of $82(18 \%)$ calves in that study were cut a second time and one of those 15 calves was cut a third time.

Recent studies in cattle suggest that bleed out is improved when the carotid arteries in the neck are severed at the position of the first cervical vertebrae (C1) instead of $\mathrm{C} 2$ to C4 (Gregory et al., 2012a, Gibson et al., 2015). Multiple reasons have been proposed to why bleeding out may be improved when the neck is cut at the $\mathrm{C} 1$ position. Firstly, there is more branching at the $\mathrm{C} 1$ position from the common carotid artery and the risk of false aneurysms sealing all severed branches may be lower than for sealing the single common carotid at the C2 to C4 position (Dyce et al., 2009). Secondly, it was proposed that there may be less stretching of the artery at the $\mathrm{C} 1$ position when the chin is lifted. When the arteries are cut at that position there is less retraction within the connective tissue sheath, decreasing the risk for occlusions and thus impaired bleeding (Gregory et al., 2012a)

The type of restraining box used and the position of the cattle in it may also influence the time to loss of consciousness. The difference in time to loss of consciousness after the neck cut between inverted and upright positioned calves was $50 \mathrm{~s}$ in the study described in Chapter 5. Bleeding out in calves slaughtered in upright position was often obstructed when the head fell limp against the head restrainer. Whether an inverted or upright position should be preferred in terms of time to loss of consciousness remains debated (Slaughter of Animals (prevention of cruelty) Regulations 1958 - quoted by Dunn (1990) (Dunn, 1990, von Holleben et al., 2010, FAWC, 2012). For animal welfare reasons (e.g. blood aspiration, rumen pressure on the diaphragm) some countries (e.g. UK) have prohibited non-stunned slaughter in an inverted position (Dunn, 1990; Petty et al., 1994; Tagawa et al., 1994).

In order to reduce the conscious period in non-stunned slaughtered animals, certain pre-cut stunning methods that do not irreversibly damage the heart or brain can be considered (Nakyinsige et al., 2013). Post-cut stunning methods are also being considered for halal slaughter and are already implemented in some slaughter plants (Farouk, 2013). Post-cut captive bolt stunning was applied in the study described in Chapter 5 and all 
cattle that had not lost consciousness due to bleeding at that time, lost consciousness within one second following the stun.

Though the conscious period following the cut may be reduced using a different bleeding technique, it does not solve the debate around pain and distress experienced by animals slaughtered without prior stunning (Gregory et al., 2012b). Opinions on pain perception during neck cutting are divided. Grandin (1994) states that when the neck cut is performed using a long, straight, razor-sharp knife it does not appear to be painful. Rosen (2004) discusses physiological aspects of Shechita, the Jewish method of religious animal slaughter and concludes that Shechita is a painless and humane method of animal slaughter. Others (e.g. Gibson et al., 2007; Gibson et al., 2009a; Mellor et al., 2009) state that the ventral neck cut is a noxious stimulus and that there is strong evidence that this cut would be perceived as painful from the time of the neck cut until loss of consciousness. The general opinion is that slaughter by neck incision of conscious animals causes pain and that bleeding while conscious may distress the animal (Gregory, 2005a; Johnson et al., 2012; Johnson et al., 2015).

Taken together, consciousness in sheep slaughtered without prior stunning is lost 2 to $20 \mathrm{~s}$ after the neck cut, with little variation between animals. Consciousness in cattle is lost seconds up to five minutes after the neck cut. Risks for impaired bleeding and thus potential prolonged consciousness involve technical skills of the operator and especially in cattle the occurrence of occlusions. Non-stunned slaughter has been associated with pain of the cut and distress during the bleeding phase, especially when the animal is cut and bled in an inverted position.

\section{EVALUATING DIFFERENT INDICATORS FOR THE ASSESSMENT OF UNCONSCIOUSNESS AT SLAUGHTER}

Abattoir employees that perform the stunning, neck cut, shackling and/or hoisting should check and confirm that animals are not conscious following stunning or before release from restraint when animals are slaughtered without prior stunning (Council Regulation (EC) 1099, 2009). Assessing the effectiveness of the stun and ensuring unconsciousness before shackling and hoisting is needed to avoid unnecessary pain and distress in the animal (Grandin, 2010). Under experimental conditions the assessment of brain function can be applied to determine the state of consciousness in an animal. The first part of this section evaluates the use of EEG activity as a method to assess unconsciousness. Under commercial conditions, readily observable indicators (e.g. behavioural indicators, physical signs and reflexes) are used to assess the state of consciousness. Some literature already looked into these different indicators and the pros and cons to their use at slaughter (e.g. Erasmus et al., 2010a; Verhoeven et al., 2015a; Terlouw et al., 2016b). In the second part 
of this section, emphasis is therefore placed on the different physical signs and reflexes evaluated in this thesis and their relationships with EEG activity. In the third part of this section, the possibilities for combining sets of indicators to increase the accuracy with which unconsciousness can be assessed at the slaughter plant are looked into.

\section{The use of the Electroencephalogram (EEG) as a method to assess unconsciousness}

The electroencephalogram (EEG) has been used to investigate electrical currents in the brain for over a century. Richard Caton and Adolf Beck are considered pioneers in the field of electroencephalography (EEG) in animals since the late $19^{\text {th }}$ century, together with Hans Berger who later applied the method to humans (Teplan, 2002; Coenen et al., 2014). The use of brain activity (e.g. evoked potentials, EEG and ECoG activity) to assess unconsciousness during stunning and slaughter was introduced much later and has been well documented since the late 1970s (Hoenderken, 1978; Lambooy, 1981; Gregory and Wotton, 1984; Lowe et al., 2007). The EEG measures electrical activity in the brain which characteristics change with different states of (un)consciousness (Lopes da Silva, 1982). The EEG trace of an awake animal will consist of a low-amplitude, high frequency signal, indicating alert animals (Verhoeven et al., 2015b; Verhoeven et al., 2016a; Verhoeven et al., 2016b). When high-amplitude, low frequency dominates the EEG trace this is interpreted to indicate unconsciousness (Baars et al., 2003; Verhoeven et al., 2015b; Verhoeven et al., 2016a). A flat EEG signal (<10\% of baseline amplitude) represents recorded background noise and not brain activity (Martin, 2015). Though the assessment of EEG activity may be considered most objective, there are limitations to its use and the interpretation of results. The division in stages does not readily provide definitive answers as to the exact moment of onset of human or animal unconsciousness, but merely provides guidelines (Meyer, 2015). There is only consensus that unconsciousness must set in somewhere between an EEG trace reflecting an awake animal and the occurrence of an iso-electric or flat EEG (cessation of cortical activity). In this thesis, visual appraisal of EEG traces was used in three consecutive studies (Chapter 3, 4 and 5) to determine times to loss of consciousness. Clear changes from conscious to unconsciousness could be detected with the visual assessment. Figure 1 shows an example of a representative series of $5 \mathrm{~s}$ of EEG registration during different EEG stages observed before and during non-stunned slaughter in calves, adopted from Chapter 4.

The aim of the different studies described in this thesis was to define objective cut-of points to discriminate between the different conscious states. The only study during which this was possible was the study described in Chapter 3 when sheep were anaesthetised with propofol. Fast Fourier Transformation (FFT) analysis of clean 2 second epochs was applied to the data to show the frequency composition of the signal during the epoch and calculate spectral variables such as total power (PTot) and Spectral Edge 


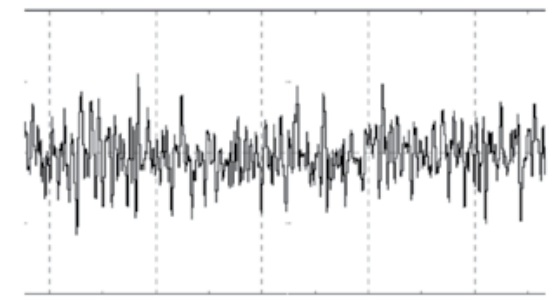

A

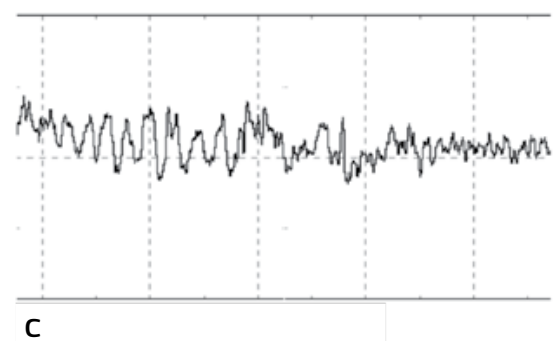

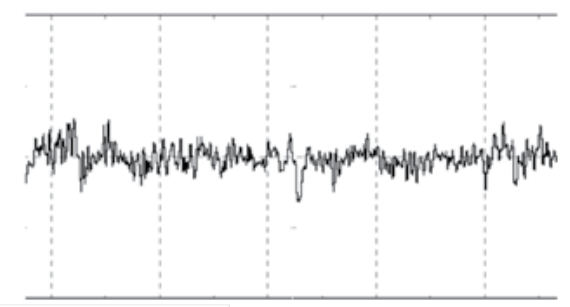

B

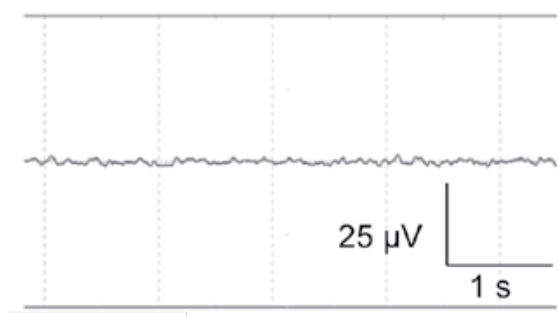

D

FIGURE IA-D

Representative examples of the different stages identified with visual assessment of electroencephalogram (EEG) activity before and after stunned and non-stunned slaughter in calves (adopted from Chapter 4). The four stages from left to right and top to bottom: baseline (a), transitional (b), unconscious (c), and minimal brain activity (d). Total X-axis represents 5 seconds, Y-axis represents amplitude of the EEG-trace $(\mu \mathrm{V})$.

Frequency (F95) per epoch. The PTot is the complete area underneath the frequency spectrum curve, the F95 is the frequency below which $95 \%$ of the power is located. These readout parameters are considered useful and standardized quantitative tools to uniformly describe changes in EEG activity (Murrell and Johnson, 2006). When during propofol anaesthesia a clear decrease or increase was detected in EEG activity, corresponding FFT values were used to determine absolute values as cut-off points. When for instance the PTot was a four-fold higher and the F95 was $8 \mathrm{~Hz}$ lower compared to baseline values this was noted as the start of EEG-unconscious. There are no general criteria, however, for defining these thresholds.

To minimize subjective interpretation of EEG data, commercial EEG monitors can be used. Commercial EEG monitors are for instance used to assess depth of anaesthesia by applying automatic transformation of raw EEG data into specific readout parameters (Bruhn et al., 2006). Interpretation of such readout parameters is often based on the data and findings of thousands of patients. Such an application sounds promising and has been used incidentally in research on stunning and slaughter (e.g. Lambooij et al., 2012; Llonch et al., 2013). Results indicated, however, that such a monitor has limited possibilities for use in commercial slaughter plants. Llonch et al. (2011) observed for instance an update delay of around $10 \mathrm{~s}$ between the state of awareness and the monitor recording. 
The recording and interpretation of EEG activity for the assessment of unconsciousness during stunning and slaughter procedures has some disadvantages to its use, limiting the applicability under commercial conditions. First, it is difficult to compare EEG values between species and individuals, because of animal variation caused by electrode placement, skull thickness and differences between equipment. This makes standardization for cut-of points/ thresholds more difficult. Secondly, the EEG can be disturbed by artefacts that are animal related (e.g. eye movements and muscle contractions) or technical related (e.g. cable movements, impedance fluctuation and $50 / 60 \mathrm{~Hz}$ interference) (Teplan, 2002). Experimental controlled situations provide a significantly better environment to limit these artefact sources than slaughter plants.

Several studies on stunning and slaughter report disconnected electrodes or disrupted EEG activity in 9-71\% of the animals (Velarde et al., 2002; Gerritzen et al., 2004; Lambooij et al., 2006). This risk is especially high when stunning or slaughter methods are used that cause direct damage to the head and brain (e.g. mechanical and electrical stunning), possibly damaging EEG electrodes and causing temporary or definite disturbance to the EEG signal (Erasmus et al., 2010b). Disconnected electrodes were observed in 3\% of non-stunned slaughtered calves (Chapter 4) and in 19\% of pigs stunned with $\mathrm{CO}_{2}$ (Chapter 5). The $\mathrm{CO}_{2}$ stunning procedure provided an additional challenge since animals could move freely and (extensive) muscular contractions could easily disturb the EEG signal, hence the larger percentage of disconnected electrodes. Part of the EEG traces from the different studies described in this thesis were disturbed due to movement artefacts that fell within the same frequency domain $(0-4 \mathrm{~Hz})$ as the recorded EEG activity (Delorme and Makeig, 2004, Lowe et al., 2007, Coenen et al., 2009). Martin (2015) describes the use of a novel filtering method developed to significantly reduce the loss of EEG data. This novel filtering method does not, however, provide a solution for compensating for severe movement artefacts in the trace. In the majority of the studies published in this thesis, movement artefacts made FFT analyses impossible. For this reason it was decided to visually assess the EEG traces and defining the different stages of consciousness more accurately was not possible therefore.

\section{Relationships between EEG activity and behavioural indicators, physical signs and reflexes \\ Rhythmic breathing}

Rhythmic breathing movements indicate that the corticospinal, ventral and lateral columns of the spinal cord are still functionally intact and this breathing pattern may thus indicate (a certain degree of) consciousness (Mitchell and Berger, 1975). Rhythmic breathing must immediately cease following a successful mechanical or electrical stun. Irregular breathing, in the second experiment of Chapter 3, was defined as a change in breathing from regular, normal breathing to a breathing pattern where shallow breathing, 
taking big gulps of air, breathing rapidly and apnoea's were alternated. Though named differently, it could be compared to 'loss of rhythmic breathing. During the second experiment described in Chapter 3, sheep were slaughtered without prior stunning and irregular breathing, based on recording of the breathing pattern, occurred on average 27 $\pm 12 \mathrm{~s}$ after sheep lost EEG-based consciousness.

Bleeding will affect the central nervous system (ischaemia) in a graded process from the cortex down. During the bleeding phase the brain stem may remain functional for some time after the cortex has failed and this explains why irregular breathing occurred on average half a minute later than time to EEG-based loss of consciousness. The results indicated that when regular breathing is absent in non-stunned slaughtered sheep, the animal is unconscious. In the study described in Chapter 4, recording of the breathing pattern was not possible since the experiment took place at a commercial slaughter plant. Live visual appraisal of breathing movements was also not possible under those circumstances, since calves were tightly restraint. In the study described in Chapter 5, rhythmic breathing was lost in all pigs following 80 or $95 \% \mathrm{CO}_{2}$ stunning. According to EEG activity, all pigs at that time had lost consciousness.

All findings together indicate that the absence of rhythmic (regular) breathing following $\mathrm{CO}_{2}$ stunning or non-stunned slaughter is an indicator of unconsciousness. The presence of rhythmic breathing during non-stunned slaughter is not conclusive in differentiation between consciousness and unconsciousness.

\section{Gasping}

Gasping is characterized by infrequent, high amplitude inspiratory efforts, that occur when there is severe hypoxia or ischaemia (Guntheroth and Kawabori, 1975). Gasping results from a suppression of respiratory-modulated neuronal activities of the pons and a recruitment of medullary mechanisms for gasping (St John, 2009). Atkinson et al. (2012) observed that regular gasping (more than 3 times gasping in a $10 \mathrm{~s}$ interval) was in some cases the only symptom observed after $\mathrm{CO}_{2}$ stunning in pigs and animals were considered unconscious in those cases. In other cases, gasping was related to other symptoms indicative for inadequate stunning (e.g. corneal reflex and righting reflex) and animals were likely to be conscious or had a high chance of regaining consciousness. Gasping has been indicated as a first indicator of onset of breathlessness (Blackmore and Petersen, 1981). In the study described in Chapter 5, gasping was one of the first behaviours in pigs exposed to $\mathrm{CO}_{2}$ and it was present while animals were conscious. It was also the last physical sign to disappear and observed for minutes after pigs had lost EEG-based consciousness. Raj (1999) refers to gasping as a rudimentary brainstem reflex and its absence may provide a conservative indicator of unconsciousness as it may persist long after brain activity has ceased (Newhook and Blackmore, 1982). In the study described in Chapter 5, two pigs that displayed signs of gasping after $\mathrm{CO}_{2}$ stunning 
showed an iso-electric EEG at the same time. It is likely that these gasps were indeed only rudimentary brain stem activity and not signs of recovery from the $\mathrm{CO}_{2}$ exposure.

All findings together indicate that the absence of gasping after $\mathrm{CO}_{2}$ stunning is an indicator of unconsciousness. The presence of gasping during $\mathrm{CO}_{2}$ stunning is not conclusive in differentiation between consciousness and unconsciousness.

\section{Loss of posture}

Loss of posture, the inability of the animal to remain in an initial standing or sitting position, is often used as first indicator for onset of unconsciousness and indicates that the cerebral cortex is no longer able to control posture (Raj et al., 1992; Raj and Gregory, 1996; Gerritzen and Hindle, 2009; Llonch et al., 2013). Loss of posture must immediately follow a successful stun in electrically and mechanically stunned animals.

In the study described in Chapter 5, the relationships between loss of posture and EEGbased loss of consciousness was investigated in pigs subjected to $80 \%$ or $95 \% \mathrm{CO}_{2}$. Loss of posture was on average observed in pigs subjected to $80 \%$ or $95 \% \mathrm{CO}_{2}$ at $4 \pm 9 \mathrm{~s}$ and $7 \pm 8$ s, respectively, before EEG-based loss of consciousness. However, the behaviour was only observed in $63 \%$ and $81 \%$ of pigs exposed to $80 \%$ or $95 \% \mathrm{CO} 2$, respectively, before EEG-based loss of consciousness. Loss of posture did thus not reflect unconsciousness in this study. A standing posture, however, always reflected consciousness in these pigs.

In captive bolt stunned calves, as described in Chapter 4, an immediate collapse was observed in some of the calves, but tight restraint of the calf often prevented collapse and made observation of this criterion difficult. Tight restraining of sheep in a hammock in the study described in Chapter 3 prevented loss of posture in all those sheep.

All findings together indicate that the loss of posture after $\mathrm{CO}_{2}$ stunning is not an indicator of unconsciousness. A standing posture in free-moving animals indicated a conscious animal, but the indicator was often difficult to observe.

\section{Involuntary muscle movements}

Terlouw et al. (2015) showed that sideways head movements or post-stun leg padding occurs in properly stunned unconscious cattle and that these movements are influenced by shot placement and skull dimensions of the animal. Neck and back movements thus occur in both unconscious and conscious animals and it can be difficult to distinguish between involuntary and voluntary movements. Following captive bolt stunning in calves, as described in Chapter 4, involuntary muscle movements could often not be observed as calves were tightly restrained. After release from restraint and during hoisting, however, such movements (e.g. neck movements, paddling of the legs, vigorous kicking) were observed, though they were not noted down. 
Muscular contractions were observed in the majority of pigs exposed to high $\mathrm{CO}_{2}$ concentrations and it has been heavily debated whether they occur before (Velarde et al., 2007; Rodriguez et al., 2008) or after (Hoenderken, 1983; Forslid, 1987; Velarde et al.; 2007) animals have lost consciousness. In the study described in Chapter 5, muscular contractions were observed before EEG-based loss of consciousness in $88 \%$ and $95 \%$ of pigs exposed to $80 \%$ and $95 \% \mathrm{CO}_{2}$, respectively.

All findings together indicate that the absence or presence of involuntary muscular movements is not conclusive in differentiation between consciousness and unconsciousness.

\section{Threat reflex}

When testing the threat reflex as applied in the studies from Chapter 3 and 4, a finger would suddenly approach the eye and a conscious animal would close its eye or withdraw its head. The reflex involves higher-order brain mechanisms, since cortical functions integrate the information coming from the environment (Liu and Ronthal, 1992). In the study described in Chapter 3, only 7 out of 21 sheep slaughtered without prior stunning, showed a threat reflex during the bleeding phase and the reflex was lost on average $7 \pm 1 \mathrm{~s}$ after the neck cut. Since sheep were considered unconscious at $15 \pm 4$ s post neck cut, absence of the threat reflex did not indicate unconsciousness in these animals. Similar results were observed in Chapter 4, were over $90 \%$ of the non-stunned slaughtered calves lost the threat-reflex before EEG based loss of consciousness. A problem that can occur when testing this reflex is that when animals are positioned in an inverted position (on their backs), the reflex cannot always be assessed because blood may run into the eyes of these animal.

All findings together indicate that with regards to non-stunned slaughter, absence of the threat reflex is not conclusive in differentiating between consciousness and unconsciousness. The presence of the threat reflex is an indicator of consciousness.

\section{Withdrawal reflex}

The withdrawal- or pain reflex has been tested in numerous way to assess unconsciousness at slaughter e.g. pinching the ear (Chapter 3), pinching the comb (Erasmus et al., 2010a), pinching the nose (Chapter 4), single needle stimulus in the skin (Limon et al., 2010) or spraying with hot water (Parotat et al., 2015). Certain responses to painful stimuli require cortical input and are thus indicative of consciousness. Other responses to painful stimuli may be simple nociceptive arc-reflex responses, based on a circuit involving the spinal cord, but not the brain (Terlouw et al., 2016b). This may explain the different results found in literature and this thesis.

Terlouw et al. (2015) observed that in response to a skin cut and sticking, some unconscious animals showed a withdrawal reflex. During propofol anaesthesia, sheep often lost the withdrawal reflex after they lost consciousness and regained it often before 
regaining consciousness. It is expected that in these cases, the withdrawal reflex is based on a neural circuit that passes through the spinal cord, but not the brain. Also in some of the calves slaughtered without prior stunning, the withdrawal reflex was lost after EEG-based unconsciousness. In all sheep (Chapter 3) and the majority (98\%) of the calves (Chapter 4) that were slaughtered without prior stunning, the withdrawal reflex was lost before the animals were considered unconscious based on EEG activity. A possibility is that transecting soft tissues (e.g. muscle, nerves, connective tissue) will cause a major amount of neural impulses to travel to the brain. This produces an intense, but brief injury discharge in the afferent nerves. The cut end of these nerves depolarise and are unable to respond to further stimuli. The massive stimulation of all sensory nerves after the neck cut can lead to a state of shock and distress (Gregory, 1998 and 2005b). Therefore, animals will probably not respond to another and milder source of pain (Johnson et al., 2012). Parotat et al. (2015) found that the finishing pigs that responded to hot-water spraying after stunning and sticking often showed a righting reflex, positive corneal reflex and sometimes even vocalized, indicating some degree of consciousness. But only one third of the pigs that showed movements in response to hot water spraying responded to a nasal septum pinch. At this stage, the discriminatory power of this reflex is uncertain and still needs further investigation (Terlouw et al., 2016b).

All findings together indicate that the absence or presence of the withdrawal reflex is not conclusive in differentiation between consciousness and unconsciousness.

\section{Eye reflexes}

Testing the corneal reflex is a generally used indicator for assessing unconsciousness in commercial slaughter plants. In the human medical world, absence of the corneal reflex is considered a worrying sign of brainstem damage (Cruccu et al., 1997). When the corneal reflex cannot be evoked, there is a large probability that the disruption is associated with a wider dysfunction, comprising part of the reticular formation, and thus with a state of unconsciousness (Serrats et al., 1976; Cruccu et al., 1997; Laureys, 2005; Terlouw et al., 2016b).

In the study described in Chapter 5, none of the pigs subjected to $80 \%$ or $95 \% \mathrm{CO}_{2}$ had a corneal reflex following stunning. Brain activity at the time of testing had also ceased in all pigs. On the opposite, dysfunction of the reticular formation at a remote distance from the neural circuit of corneal reflex, or interruption of the ascending reticular activating system can both induce a state of unconsciousness without abolishing the corneal reflex (Terlouw et al., 2016b). This implies that an unconscious animal at slaughter can still show a corneal reflex. Anil (1991) observed return of reflexes after electrical stunning in finishing pigs and observed that the corneal reflex returned on average $48 \mathrm{~s}$ after the stun. Pigs were at that time considered unconscious as the response to a nose prick and return of righting reflex returned later in these animals. The corneal reflex was 
lost in non-stunned slaughtered calves (Chapter 4) $85 \pm 45 \mathrm{~s}$ after EEG-based loss of consciousness and in $90 \%$ of the recordings, all brain activity had ceased at that stage. When slaughtering livestock without prior stunning, absence of the corneal reflex must be interpreted with care, since in $9 \%$ of the calves in Chapter 4 , the reflex was lost and regained during the bleeding phase. Return of the corneal reflex has been observed by Hoffman in an unspecified number of cattle (Hoffman, 1900 cited by Gregory et al., 2010). Bourguet et al. (2011) also observed a temporary return of the eyelid- and corneal reflex in some of the non-stunned slaughtered cattle. A temporary return of blood pressure would be necessary for these reflexes to return and may possibly be explained by physiological changes, such as an increased heart rate, to alleviate the drop in blood pressure (Newhook \& Blackmore, 1982; Vimini et al., 1983). These signs are not necessarily indicative of a partial or total return of consciousness, but leave a doubt (Gregory et al., 2010).

Under certain circumstances, the testing of eye reflexes may be difficult. Blood may run in the eyes during the bleeding phase, especially in inverted animals, which limits the possibility to assess the reflex. Eyeball rotation may also limit the possibility to test the corneal reflex as the cornea cannot be reached (Gouveia et al., 2009; Bourguet et al., 2011). Blackmore and Delany (1988) describe the absence of the eyelid reflex as indicator for approaching brain death or severe brain impairment, as it can still be elicited under deep anaesthesia. This was also observed in the study described in Chapter 3, where the eyelid reflex was the last reflex to be lost and the first to be regained in sheep anaesthetised with propofol. In that particular experiment, the reflex was not lost once in 8 of the 20 observations. Its absence indicated unconsciousness, but the presence of the eyelid reflex did not necessarily indicate consciousness. In non-stunned slaughtered sheep, the eyelid reflex was lost on average $60 \pm 14 \mathrm{~s}$ after EEG based loss of consciousness. The moment the eyelid reflex was lost for the first time, the EEG had turned iso-electric in 20 out of 21 sheep. Similar results were observed in non-stunned slaughtered calves, were the eyelid reflex was observed until $76 \pm 50 \mathrm{~s}$ after EEG-based loss of consciousness (Chapter 4 ).

All findings together indicate that the absence of the corneal- and eyelid reflex are indicators of unconsciousness. The presence of the corneal- and eyelid reflex during non-stunned slaughter are not conclusive in differentiation between consciousness and unconsciousness. Table 5 shows a summary of the main findings on the use of different indicators that were applied to assess unconsciousness at slaughter in this thesis.

Overall, indicators for unconsciousness as observed in this thesis included absence of rhythmic breathing, absence of the eyelid reflex and absence of the corneal reflex. Presence of the threat reflex indicated consciousness. Absence and presence of the remaining indicators were not conclusive in differentiating between consciousness and unconsciousness. 
TABLE 5

Main findings on the use of different indicators applied in this thesis to assess unconsciousness at slaughter.

\begin{tabular}{|c|c|c|c|}
\hline & Presence & Absence & \\
\hline Indicator & $\begin{array}{l}\text { Animal } \\
\text { unconscious }\end{array}$ & $\begin{array}{l}\text { Animal } \\
\text { unconscious }\end{array}$ & Remarks \\
\hline Rhythmic breathing & Inconclusive & Yes & $\begin{array}{l}\text { Difficult to observe under commercial } \\
\text { conditions, especially when the animal is } \\
\text { restrained }\end{array}$ \\
\hline Gasping & Inconclusive & Inconclusive & \\
\hline Loss of posture & Inconclusive & Inconclusive & $\begin{array}{l}\text { Cannot be assessed when the animal is } \\
\text { restrained }\end{array}$ \\
\hline $\begin{array}{l}\text { Involuntary muscle } \\
\text { movements }\end{array}$ & Inconclusive & Inconclusive & $\begin{array}{l}\text { Difficult to distinguish between involuntary } \\
\text { and voluntary movements }\end{array}$ \\
\hline Threat reflex & No & Inconclusive & \\
\hline Withdrawal reflex & Inconclusive & Inconclusive & \\
\hline Corneal reflex & Inconclusive & Yes & \\
\hline Eyelid reflex & Inconclusive & Yes & \\
\hline
\end{tabular}

\section{Other indicators for unconsciousness}

Besides the above mentioned indicators, some other potential indicators for assessing unconsciousness at slaughter have been described. More research is needed to investigate how accurate they are as indicators for unconsciousness under commercial conditions. Examples of such indicators include a floppy or limp head (Grandin, 2002), protruding tongue (Grandin, 2002), jaw relaxation (Gregory et al., 2009), convulsions and muscle tone (Terlouw et al., 2016b), nystagmus (Gregory et al., 2007; Terlouw et al., 2015), eye ball rotation (Gregory et al., 2007; Atkinson et al., 2013) and feather erection in birds (Erasmus et al., 2010a; Terlouw et al., 2016b).

\section{Combining multiple behavioural indicators, physical signs and reflexes to assess unconsciousness}

The differences in sensitivity to anoxia of different parts of the brain may explain the order in which different reflexes are lost (Terlouw et al., 2016b). Cortical regions are the most sensitive to oxygen want, where the medulla of the brainstem is often the last structure to lose its function in the way to brain death (Van Liere and Stickney, 1963; Wijdicks, 2001). When looking into the data from the studies as described in this thesis, some consistencies in loss and regain of specific indicators were observed. In general, the order of loss of indicators for assessing unconsciousness in non-stunned slaughtered animals was: loss of withdrawal reflex, loss of response to threat, EEG-based loss of consciousness, loss of rhythmic breathing, loss of eyelid reflex and loss of corneal reflex. 
The different studies from this thesis, combined with literature, however, also revealed that rhythmic breathing, the corneal- and eyelid reflex were all lost long after animals were considered unconscious based on EEG activity, making them distinctly conservative indicators in the assessment of unconsciousness.

It has been stated that the use of multiple indicators provides a more reliable assessment of unconsciousness at slaughter than the use of a single indicator (Erasmus et al., 2010a; EFSA, 2013c and 2013b; Verhoeven et al., 2015a; Terlouw et al., 2016b). From the results described in Chapter 5, it can be concluded that absence of the corneal reflex or absence of rhythmic breathing reflected unconsciousness following $\mathrm{CO}_{2}$ stunning in pigs. Both indicators were assessed only when pigs had returned from the long stay down in the well and since both indicators were absent at that time, combining the two indicators would not provide additional or more accurate information.

From the results described in Chapter 3, 4 can be concluded that absence of rhythmic breathing, absence of the eyelid reflex or absence of the corneal reflex reflected unconsciousness in non-stunned slaughtered animals. Since none of the other indicators from Table 5 were conclusive in their answer to whether an animal was unconscious or not, combining other indicators will not provide additional or more accurate information.

\section{MAIN CONCLUSIONS}

In conclusion, results described in this thesis indicate that captive bolt stunning instantly induces unconsciousness. Loss of consciousness is not instant during $\mathrm{CO}_{2}$ stunning, but may take up to 1 minute, depending on the $\mathrm{CO}_{2}$ concentration used. An increased $\mathrm{CO}_{2}$ concentration decreases the time to loss of consciousness. Consciousness in sheep slaughtered without prior stunning is lost on average $15 \mathrm{~s}$ after the neck cut, while in cattle, times to loss of consciousness varied from $14 \mathrm{~s}$ up to two minutes.

The results further show that absence of rhythmic breathing, corneal reflex- and eyelid reflex are valid indicators of unconsciousness. The result of assessing absence of these indicators at slaughter and (re)stunning when present will, however, imply that many animals need (re)stunning. In a proportion of those (re)stunned animals, consciousness will be lost at the time of (re)stunning.

The current thesis also shows that under full commercial conditions, stunning effectiveness must be closely monitored by slaughter plant employees, since many factors can influence the slaughter process and increase the risks for stun failures. 


\section{PRACTICAL IMPLICATIONS}

Stunning animals to render them unconscious is mandatory prior to bleeding. To comply with this EU legislation, different stunning methods are applied on livestock (e.g. mechanical-, electrical- and gas stunning). In animals subjected to particular methods of slaughter prescribed by religious rites, consciousness is lost due to bleeding. It is critical to determine the onset of unconsciousness for all methods applied, since only animals that are unconscious will not experience pain or distress. The most objective method to assess unconsciousness is by assessing brain activity, though under full commercial conditions this method cannot be applied. In the different studies described in this thesis the use of brain activity, as presented in an EEG, was used to study the times to loss of consciousness for different stunning and slaughter methods. From the different methods described in this thesis, captive bolt stunning was the only method that instantly induced unconsciousness. It therefore prevails as the stunning method of choice over the other stunning and slaughter methods studied in this thesis.

It should be noted, however, that the majority of the studies described in this thesis were performed under either experimental conditions or semi-commercial conditions. Full commercial conditions provide a whole different setting in terms of line speed, working safety and food hygiene regulations. Under commercial conditions there is a higher risk for stun failures than during the studies described in this thesis. Risks for stun failures under commercial circumstances can be technical related (e.g. insufficient current intensity, misplacement of electrodes or insufficient exposure time) or operator related (e.g. skills and experience of slaughter plant employees, poor maintenance of equipment). To minimize risks for stun failures, additional checks or critical control points can be added to slaughter plant procedures. Grandin (2013) and the European Food Safety Authority (EFSA) have written multiple protocols on recommended handling, guidelines and audit methods than can be applied in slaughter plants. Recording and assessing EEG activity was in this thesis applied under semi-commercial conditions, meaning that it was applied in slaughter plants (Chapter 4 and 5), but that the line speed was decreased during those studies. Its use, however, shows that the method can be used as an audit tool in slaughter plants when at times there is the need to evaluate stunning and slaughter practices more in depth.

Since the slaughter process can be influenced or disturbed by many external factors there always remains the possibility for animals to be conscious at the time of neck cut or while bleeding. To minimize the frequency of such incidences, one should check each animal for unconsciousness during multiple stages of the slaughter process (following stunning, prior to the neck cut, prior to release from restraint and during the bleeding phase). Since the use of EEG activity is limited to experimental or semi-commercial conditions, one must rely on readily observable indicators (e.g. behavioural indicators, physical signs and 
reflexes). It has been debated which of these readily observable indicators are most accurate in assessing unconsciousness. The results from this thesis showed that there are indicators of which the absence always indicate unconsciousness in animals that lose consciousness gradually. Following $\mathrm{CO}_{2}$ stunning these indicators are absence of rhythmic breathing and absence of the corneal reflex. During bleeding in non-stunned slaughtered animals, these indicators are absence of rhythmic breathing and absence of the cornealor eyelid reflex.

If the presence of rhythmic breathing, corneal reflex or eyelid reflex would be the requirement to re-stun animals and we would apply this requirement to the data of the studies on $\mathrm{CO}_{2}$ stunning (Chapter 5) and captive bolt stunning (Chapter 4), none of the animals would need to be re-stunned. It should be noted, however, that the pigs immersed in $\mathrm{CO}_{2}$ (Chapter 5) remained there for much longer (346 s) than is commonly applied in practice (92-238 s) (Velarde et al., 2000; Atkinson et al., 2016). Under full commercial $\mathrm{CO}_{2}$ stunning conditions animals would have a higher chance of displaying the corneal reflex and thus for the need to be re-stunned following that criterium (Table 2).

It has been formally agreed in The Netherlands that animals subjected to non-stunned slaughter should be unconscious within $40 \mathrm{~s}$, based on absence of at least 3 of the following 5 indicators: (1) threat reflex; (2) withdrawal reflex; (3) corneal reflex; (4) (spontaneous) eyelid reflex; (5) rhythmic breathing (Staatscourant, 2012). Rhythmic breathing can often not be observed due to tight restraint of the animal. Both threat- and withdrawal reflex were regularly absent when the animal was still conscious. Those three indicators are therefore not recommended for definitively assessing unconsciousness in animals subjected to non-stunned slaughter. Absence of the eyelid- and corneal reflex are recommended, as it was shown that they are indicative of unconsciousness. If the presence of one of those two indicators would thus be the requirement to stun animals at $40 \mathrm{~s}$ and we would apply this requirement to the data of the studies on non-stunned slaughtered sheep (Chapter 3) and calves (Chapter 4) it would have had the following implications for our studies. All of the sheep slaughtered without prior stunning had lost consciousness at $40 \mathrm{~s}$ based on EEG activity (Chapter 3). The eyelid reflex, however, was still present at that time in 20 of 21 sheep. Based on the absence of this indicator, 95\% of the sheep should have been stunned at $40 \mathrm{~s}$.

None of the calves $(n=7)$ slaughtered in an upright position had lost consciousness at 40 s, based on EEG activity (Chapter 4). Both eyelid- as well as corneal reflex were present in all calves at that time. Based on the absence of those indicators, $100 \%$ of those calves should have been stunned at $40 \mathrm{~s}$.

Eleven of the calves $(n=24)$ slaughtered in an inverted position had lost consciousness at $40 \mathrm{~s}$, based on EEG activity (Chapter 4). Both eyelid- as well as corneal reflex were present in all calves at that time. Based on the absence of those indicators, $100 \%$ of the calves should have been stunned at $40 \mathrm{~s}$. 
APPENDICES 


\section{SUMMARY}

\section{REFERENCES}

\section{ACKNOWLEDGEMENTS}

ABOUT THE AUTHOR

TRAINING AND SUPERVISION PLAN

COLOPHON

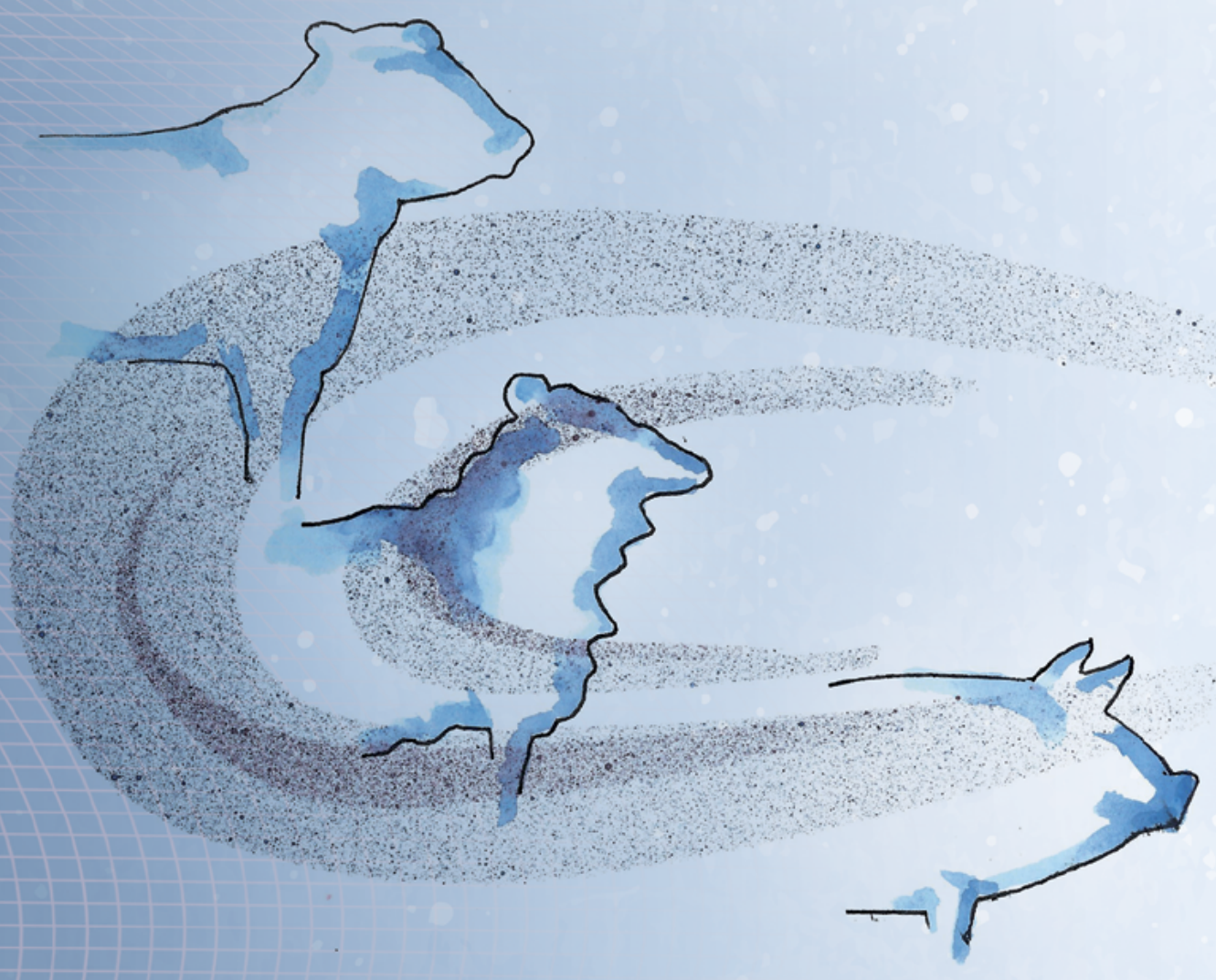




\section{SUMMARY}

European legislation provides laws, rules and procedures regarding the slaughter of livestock. One of these laws states that animals should be stunned prior to bleeding to render them unconscious, since animals that are unconscious will not experience pain or distress. Abattoir employees are required to assess and confirm unconsciousness after stunning before further processing (neck-cut, shackling and hoisting) takes place. In animals subjected to methods of slaughter prescribed by religious rites, unconsciousness must be ascertained before release from restraint. For all stunning and slaughter methods, it is thus critical to determine the onset and duration of unconsciousness.

Unconsciousness at slaughter is generally assessed using readily observable indicators (e.g. behavioural indicators, physical signs and reflexes). There is, however, substantial (inter)national debate about which indicators most adequately assess unconsciousness at slaughter and which merit further investigation. Under experimental conditions, the assessment of brain activity, as presented in an electroencephalogram (EEG), can be used to provide information on the state of consciousness. Knowledge derived from studies performed under experimental conditions in which the relationships between both brain activity and readily observable indicators are assessed, could lead to better validated and applicable indicators to reliably and reproducibly assess unconsciousness at slaughter. As a result, the risk of continuing the slaughter process in a conscious animal can be decreased.

The aim of this thesis was to study times to loss of consciousness and the relationships between behavioural indicators, physical signs, reflexes and brain activity, as presented in an EEG, in different species subjected to different stunning and slaughter methods.

In Chapter 2, the different indicators (e.g. behavioural indicators, physical signs, reflexes and brain activity) available to assess unconsciousness after stunning under both commercial and experimental conditions were reviewed. The review showed that the applicability and reliability of the different indicators vary per stunning method. It was concluded that it is recommendable to use a set of indicators rather than a single indicator to assess unconsciousness before continuation of the slaughter process.

The experiments described in Chapter 3, 4 and 5 investigated times to loss of consciousness as well as the relationships between behavioural indicators, physical signs, reflexes and the time to onset of EEG stages (e.g. transitional, unconscious or iso-electric) representing different conscious states in multiple species subjected to different stunning and slaughter methods.

During propofol anaesthesia in sheep (Chapter 3), consciousness was lost on average 33 $\pm 5 \mathrm{~s}$ after start administrating propofol. A rhythmic breathing pattern, as a result of the effect of the anaesthetic agent on the respiratory control centre, and absence of the threat reflex indicated unconsciousness. The use of the eyelid- and withdrawal reflex in the 
assessment of unconsciousness was often not conclusive. Both reflexes were present in animals that were considered unconscious based on EEG activity. Following non-stunned slaughter in these animals, consciousness was lost, based on EEG activity, after on average $15 \pm 4$ s. Only seven of the 21 sheep showed a threat reflex after the neck cut and this reflex was always lost before EEG-based unconsciousness. The withdrawal reflex was never present following the neck cut during the bleeding phase. Regular breathing and the eyelid reflex were on average lost $43 \pm 12 \mathrm{~s}$ and $74 \pm 17 \mathrm{~s}$, respectively, after the neck cut when all sheep were unconscious based on EEG activity.

To verify whether the results in sheep described in Chapter 3 would be similar in cattle, relationships between onset of different EEG stages and multiple readily observable indicators (threat-, withdrawal-, corneal-, and eyelid reflex) were assessed and subsequently described in Chapter 4. Calves in lairage were randomly assigned to one of four treatments namely: captive bolt stunning, slaughter without stunning in an upright or an inverted position or slaughter without stunning in an upright position followed by captive bolt stunning $40 \mathrm{~s}$ after the neck cut. Calves were considered unconscious based on EEG activity immediately ( $1 \mathrm{~s}$ ) after captive bolt stunning. In these calves, reflexes were all permanently lost at the first testing moment (5s) after stunning. Calves slaughtered without stunning in either an upright or inverted position were unconscious, based on EEG activity, $109 \pm 32$ and $49 \pm 25 \mathrm{~s}$, respectively, after the neck cut. In those animals, the threat- and withdrawal reflex were lost before calves were unconscious based on EEG activity. The corneal- and eyelid reflex were lost after calves were unconscious based on EEG activity. The latter two indicators appeared to be distinctly conservative measures of unconsciousness, since they were observed until $76 \pm 50 \mathrm{~s}$ and $85 \pm 45 \mathrm{~s}$, respectively, after EEG-based unconsciousness.

In the experiment described in Chapter 5, the relationships between onset of different EEG stages and concomitant behaviour was assessed in pigs assigned to $80 \% \mathrm{CO}_{2}(80 \mathrm{C})$ or $95 \% \mathrm{CO}_{2}$ (95C) stunning. The $\mathrm{CO}_{2}$ exposure process lasted $346 \mathrm{~s}$ after which the corneal reflex and rhythmic breathing were assessed for one minute, but were not observed in any of the pigs. Time to unconsciousness was longer in $80 \mathrm{C}$ pigs (47 $\pm 6 \mathrm{~s}$ ) than in $95 \mathrm{C}$ pigs ( $33 \pm 7 \mathrm{~s}$ ). Loss of posture was observed both before and after pigs were unconscious based on EEG activity, but occurred from all scored behaviours most closely to EEG-based loss of consciousness. None of the behaviours that were scored during $\mathrm{CO}_{2}$ exposure could indicate loss of consciousness accurately. During exposure to the gas, both $80 \mathrm{C}$ and 95C pigs exhibited similar aversive behaviours before loss of consciousness. 95C pigs exhibited these behaviours on average earlier than $80 \mathrm{C}$ pigs. Furthermore, $88 \%$ of the $80 \mathrm{C}$ pigs and $94 \%$ of the $95 \mathrm{C}$ pigs demonstrated muscular contractions before unconsciousness. Both aversive behaviours and muscular contractions may comprise animal welfare. The interval between onset of the different behaviours and unconsciousness and the length of the period during which these behaviours were 
observed, except gasping, was similar for both treatments. The results provided evidence to conclude on a behavioural basis that the two atmospheres (80C and 95C) are similar in their impact on pig welfare.

In Chapter 6 it was discussed that the slaughter process is guided by societal acceptance which as such has a clear ethical component. The way in which we view animals has changed dramatically over time and has resulted in far-reaching and comprehensive legislation that regulates the treatment of animals on farm, during transport and at the slaughterhouse. But even with legislation on animal welfare in place, there still is a plurality in views on the moral status of animals and on the acceptability of the practice of slaughter. Understanding the origin of this plurality in views can improve the quality of debates regarding such topics. Ethical reflection within, and empowerment of, parties in the sector is therefore needed. New scientific research may influence someone's view on the ethical acceptability of the slaughter practice. It is therefore important for all parties involved to be aware of the recent scientific developments regarding such topics. We analysed more in detail the plurality in moral views with regard to the slaughter process. Second, we presented recent developments in scientific research on the stunning of animals. Finally, we explored how this scientific research can contribute in the ethical debate on the acceptability of the current slaughter process. In Chapter 7, the general discussion, times to loss of consciousness, risks for stun failures and animal welfare concerns related to the most commonly used stunning methods were discussed. In the second section, the presence and absence of different indicators and their relationships with EEG activity were evaluated. The last part of Chapter 7 described the practical implications of the work described in this thesis.

In conclusion, results described in this thesis indicate that captive bolt stunning instantly induces unconsciousness. Loss of consciousness is not instant during $\mathrm{CO}_{2}$ stunning, but may take up to 1 minute, depending on the $\mathrm{CO}_{2}$ concentration. An increased $\mathrm{CO}_{2}$ concentration decreases the time to loss of consciousness. Consciousness in sheep slaughtered without prior stunning is lost on average $15 \mathrm{~s}$ after the neck cut, while in cattle, time to loss of consciousness may take up to two minutes.

Absence of rhythmic breathing, corneal reflex- and eyelid reflex are valid indicators of unconsciousness following captive bolt stunning, $\mathrm{CO}_{2}$ stunning and non-stunned slaughter. These indicators are, however, quite conservative and therefore imply that when presence of any of these indicators would be the requirement for (re)stunning, many animals need (re)stunning under current commercial practices. In a proportion of those (re)stunned animals, consciousness will be lost at the time of (re)stunning. Results also show that under full commercial conditions, stunning effectiveness must be closely monitored by slaughter plant employees, since many factors can influence the slaughter process and increase the risks for stun failures and thus prolonged consciousness. 


\section{REFERENCES}

A

Adams, D.B. and Sheridan, A.D. 2008. Specifying the risks to animal welfare associated with livestock slaughter without induced insensibility. Retrieved on 19 November 2013 from http://www.australiananimalwelfare.com.au/app/webroot/files/upload/files/animalwelfare-livestock-slaughter.pdf.

Alkire, M.T., Hudetz, A.G. and Tononi, G. 2008. Consciousness and anesthesia. Science 322, 876-880.

Anastasov, M. and Wotton, S. 2012. Survey of the incidence of post-stun behavioural reflexes in electrically stunned broilers in commercial conditions and the relationship of their incidence with the applied water-bath electrical parameters. Animal Welfare 21, 247-256.

Anil, M.H. 1991. Studies on the return of physical reflexes in pigs following electrical stunning. Meat Science 30, 13-21.

Anil, M. and McKinstry, J. 1991. Reflexes and loss of sensibility following head-to-back electrical stunning in sheep. The Veterinary Record 128, 106-107.

Anil, M. and McKinstry, J. 1998. Variations in electrical stunning tong placement andrelative consequences in slaughter pigs. The Veterinary Journal 155, 85-90.

Anil, M., Whittington, P. and McKinstry, J. 2000. The effect of the sticking method on the welfare of slaughter pigs. Meat Science 55, 315-319.

Anil, M.H., Raj, A.B.M. and McKinstry, J.L. 2000. Evaluation of electrical stunning in commercial rabbits: effect on brain function. Meat Science 54, 217-220.

Atkinson, S., Velarde, A., Llonch, P. and Algers, B. 2012. Assessing pig welfare at stunning in Swedish commercial abattoirs using CO. Animal Welfare 21, 487-495.

Atkinson, S., Velarde, A. and Algers, B. 2013. Assessment of stun quality at commercial slaughter in cattle shot with captive bolt. Animal Welfare 22, 473-481.

AVMA. 2013. AVMA Guidelines for the Euthanasia of Animals: 2013 Edition. Retrieved on July 262014 from https://www.avma.org/kb/policies/documents/euthanasia.pdf.

Avsaroglu, H., Van der Sar, A., Van Lith, H., Van Zutphen, L., Hellebrekers, L. 2007. Differences in response to anaesthetics and analgesics between inbred rat strains. Laboratory Animals 41, 337-344. 
Baars, B.J., Ramsøy, T.Z. and Laureys, S. 2003. Brain, conscious experience and the observing self. Trends in Neurosciences 26, 671-675.

Bager, F., Braggins, T., Devine, C., Graafhuis, A., Mellor, D., Tavener, A. and UpSDell, M. 1992. Onset of insensibility at slaughter in calves: effects of electroplectic seizure and exsanguination on spontaneous electrocortical activity and indices of cerebral metabolism. Research in Veterinary Science 52, 162-173.

Baldwin, B., Bell, F., 1963a. The anatomy of the cerebral circulation of the sheep and ox. The dynamic distribution of the blood supplied by the carotid and vertebral arteries to cranial regions. Journal of Anatomy 97, 203-215.

Baldwin, B., Bell, F., 1963b. The effect of temporary reduction in cephalic blood flow on the EEG of sheep and calf. Electroencephalography and Clinical Neurophysiology 15, 465473.

Barfod, K. 1990. CO 2 stunning of pigs. German Fleischwirtschaft 70, 1162-1174.

Barnett, J., Cronin, G. and Scott, P. 2007. Behavioural responses of poultry during kosher slaughter and their implications for the birds' welfare. The Veterinary Record 160, 45-49.

Beausoleil, N., Mellor, D. 2015. Introducing breathlessness as a significant animal welfare issue. New Zealand Veterinary Journal 63, 44-51.

Benson, E.R., Alphin, R.L., Rankin, M.K., Caputo, M.P., Kinney, C.K. and Johnson, A.L. 2012. Evaluation of eeg based determination of unconsciousness versus loss of posture in broilers. Research in Veterinary Science 93, 960-964.

Beyssen, C., Babile, R. and Fernandez, X. 2004. Electrocorticogram spectral analysis and somatosensory evoked potentials as tools to assess electrical stunning efficiency in ducks. British Poultry Science 45, 409-415.

Blackman, N., Cheetham, K. and Blackmore, D. 1986. Differences in blood supply to the cerebral cortex between sheep and calves during slaughter. Research in Veterinary Science 40, 252-254.

Blackmore, D. 1984. Differences in behaviour between sheep and cattle during slaughter. Research in Veterinary Science 37, 223-226.

Blackmore, D. and Petersen, G. 1981. Stunning and slaughter of sheep and calves in New Zealand. New Zealand Veterinary Journal 29, 99-102. 
Blackmore, D.K. and Newhook, J.C. 1982. Electroencephalographic studies of stunning and slaughter of sheep and calves- Part 3: The duration of insensibility induced by electrical stunning in sheep and calves. Meat Science 7, 19-28.

Blackmore, D. and Delany, M. 1988. Slaughter of stock, a practical review and guide. Publication No. 118. Veterinary Continuing Education, Massey University, Palmerston North, New Zealand.

Blackmore, D., Newhook, J. and Grandin, T. 1983. Time of onset of insensibility in fourto six-week-old calves during slaughter. Meat Science 9, 145-149.

Bourguet, C., Deiss, V., Tannugi, C.C. and Terlouw, E. 2011. Behavioural and physiological reactions of cattle in a commercial abattoir: Relationships with organisational aspects of the abattoir and animal characteristics. Meat Science 88, 158-168.

Brambell, F. 1965. Report of the Technical Committee to Enquire into the Welfare of Animals Kept Under Intensive Livestock Husbandry Systems: Presented to Parliament by the Secretary of State for Scotland and the Minister of Agriculture, Fisheries and Food by Command of Her Majesty December, 1965. HM Stationery Office.

Broom, D. 2001. Evolution of pain. Vlaams Diergeneeskundig Tijdschrift 70, 17-21.

Brown, R.E., Basheer, R., McKenna, J.T., Strecker, R.E. and McCarley, R.W. 2012. Control of sleep and wakefulness. Physiological Reviews 92, 1087-1187.

Bruhn, J., Myles, P.S., Sneyd, R. and Struys, M.M.R.F. 2006. Depth of anaesthesia monitoring: What's available, what's validated and what's next? British Journal of Anaesthesia 97, 85-94.

C

Carlson, N.R. 2007. Physiology of behaviour. Pearson Education Inc., Boston, MA, United States of America.

CBS 2014. http://statline.cbs.nl/Statweb/publication/?DM=SLNL\&PA=7123SLAC\&D1 $=0 \& D 2=a \& D 3=12,77,142,207,220,233,246,259,298,311,(1-15),(1-14),(1-13),(1-2),(1-1)$ $1,1 \& \mathrm{VW}=\mathrm{T}$ retrieved on 29th of April 2016.

Coenen, A., Fine, E. and Zayachkivska, O. 2014. Adolf Beck: A Forgotten Pioneer in Electroencephalography. Journal of the History of the Neurosciences 23, 276-286.

Coenen, A., Lankhaar, J., Lowe, J. and McKeegan, D. 2009. Remote monitoring of electroencephalogram, electrocardiogram, and behaviour during controlled atmosphere stunning in broilers: implications for welfare. Poultry Science 88, 10-19. 
Council Directive 93/119/EC 1993. 1993. Directive 93/119/EC on the protection of animals at the time of slaughter or killing. European Community Official Journal 340, 21-34.

Council Regulation 1099/2009 on the protection of animals at the time of killing. 2009. Official Journal of the European Union L 303, 1-30.

Crick, F. and Koch, C. 1995. Are we aware of neural activity in primary visual cortex? Nature 375, 121-123.

Cruccu, G., Leardi, M., Ferracuti, S. and Manfredi, M. 1997. Corneal reflex responses to mechanical and electrical stimuli in coma and narcotic analgesia in humans. Neuroscience Letters 222, 33-36.

Dalmau, A., Temple, D., Rodriguez, P., Llonch, P. and Velarde, A. 2009. Application of the Welfare Quality ${ }^{\circledast}$ protocol at pig slaughterhouses. Animal Welfare 18, 497-505.

Daly, C.C., Gregory, N.G. and Wotton, S.B. 1987. Captive bolt stunning of cattle: Effects on brain function and role of bolt velocity. British Veterinary Journal 143, 574-580.

Dalmau, A., Rodríguez, P., Llonch, P., Velarde, A. 2010. Stunning pigs with different gas mixtures: Aversion in pigs. Animal Welfare 19, 325-333.

Daly, C., Kallweit, E. and Ellendorf, F. 1988. Cortical function in cattle during slaughter: conventional captive bolt stunning followed by exsanguination compared with shechita slaughter. Veterinary Record 122, 325-329.

Daniels, N. 1979. Wide reflective equilibrium and theory acceptance in ethics. The Journal of Philosophy 76, 256-282.

Davidson, A.J. 2006. Measuring anesthesia in children using the EEG. Pediatric Anesthesia 16, 374-387.

DeGrazia, D. 1996. Taking animals seriously: mental life and moral status. Cambridge University Press.

Delgado-Garcia, J.M., Gruart, A., Trigo, J.A. 2003. Physiology of the eyelid motor system. Annals of the New York Academy of Sciences 1004, 1-9.

Delorme, A. and Makeig, S. 2004. EEGLAB: an open source toolbox for analysis of singletrial EEG dynamics including independent component analysis. Journal of Neuroscience Methods 134, 9-21. 
Devine, C., Gilbert, K., Graafhuis, A., Tavener, A., Reed, H., Leigh, P. 1986. The effect of electrical stunning and slaughter on the electroencephalogram of sheep and calves. Meat Science 17, 267-281.

DG SANCO, 2012. Study on various methods of stunning for poultry. Retrieved on $6^{\text {th }}$ of May 2014 from http://edz.bib.uni-mannheim.de/daten/edz-a/gdgv/12/study_ stunning_poultry_en.pdf

Dialrel. 2010. WP2.2. Religious slaughter: Evaluation of current practices. Retreived on September $4^{\text {th }} 2015$ from http://dialrel.eu/images/factsheet-practice.pdf.

Dodman, N. 1976. Observations on the use of the Wernberg dip-lift carbon dioxide apparatus for pre-slaughter anaesthesia of pigs. The British veterinary journal 133, 71-80.

Dugdale, A. 2010. Veterinary anaesthesia: principles to practice. Blackwell Publishing Ltd. Oxford, United Kingdom.

Dunn, C. 1990. Stress reactions of cattle undergoing ritual slaughter using two methods of restraint. Veterinary Record 126, 522-525.

Dyce, K.M., Sack, W.O. and Wensing, C.J.G. 2009. Textbook of Veterinary Anatomy. Elsevier Health Sciences.

E

EFSA, 2004. Welfare aspects of stunning and killing methods. Report EFSA-Q-2003-093 AHAW / 04-027, pp. 1-241.

EFSA, 2006. The welfare aspects of the main systems of stunning and killing applied to commercially farmed deer, goats, rabbits, ostriches, ducks, geese and quail. European Food and Safety Authority Journal 326, 1-18.

EFSA, 2012. S Scientific Opinion on the electrical requirements for waterbath stunning equipment applicable for poultry. European Food and Safety Authoroty Journal 10, 1-80.

EFSA, 2013a. Guidance on the assessment criteria for studies evaluating the effectiveness of stunning interventions regarding animal protection at the time of killing. European Food and Safety Authoroty Journal 11, 1-41.

EFSA, 2013b. Scientific Opinion on monitoring procedures at slaughterhouses for bovines. European Food and Safety Authoroty Journal 11, 1-65.

EFSA, 2013c. Scientific Opinion on monitoring procedures at slaughterhouses for pigs. European Food and Safety Authoroty Journal 11, 1-62. 
EFSA, 2014. Scientific Opinion on electrical requirements for poultry waterbath stunning equipment. European Food and Safety Authoroty Journal 12, 3745.

Erasmus, M.A., Turner, P.V. and Widowski, T.M. 2010a. Measures of insensibility used to determine effective stunning and killing of poultry. Journal of Applied Poultry Research 19, 288-298.

Erasmus, M.A., Lawlis, P., Duncan, I.J.H. and Widowski, T.M. 2010b. Using time to insensibility and estimated time of death to evaluate a non penetrating captive bolt, cervical islocation, and blunt trauma for on-farm killing of turkeys. Poultry Science 89, 1345-1354.

Erhardt, W., Ring, C., Kraft, H., Schmid, A., Weinmann, H.M., Ebert, R., et al., 1989. $\mathrm{CO}_{2}$-stunning of swine for slaughter from the anesthesiological viewpoint. Deutsche Tierarztliche Wochenschrift 96, 92-99.

Eurobarometer, 2015. Attitudes of EU citizens towards Animal Welfare. European Commission, Brussels, Belgium.

F

Farouk, M.M. 2013. Advances in the industrial production of halal and kosher red meat. Meat Science 95, 805-820.

Farm Animal Welfare Committee (FAWC). 2012. FAWC advice on cattle inversion for religious slaughter (independent report). Retrieved on 26 January 2015 from https:// www.gov.uk/government/uploads/system/uploads/attachment_data/file/325002/FAWC_ advice_on_cattle_inversion_for_religious_slaughter.pdf.

Finnie, J.W. 1995. Neuropathological changes produced by captive bolt stunning of cattle. New Zealand Veterinary Journal 43, 83-85.

Finnie, J.W., Blumbergs, P.C., Manavis, J., Summersides, G. and Davies, R. 2000. Evaluation of brain damage resulting from penetrating and non-penetrating captive bolt stunning using lambs. Australian Veterinary Journal 78, 775-778.

Finnie, J., Manavis, J., Blumbergs, P. and Summersides, G. 2002. Brain damage in sheep from penetrating captive bolt stunning. Australian Veterinary Journal 80, 67-69.

Forslid, A. 1987. Transient neocortical, hippocampal and qmygdaloid EEG silence induced by one minute inhalation of high concentration $\mathrm{CO}_{2}$ in swine. Acta Physiologica Scandinavica 130, 1-10.

Forslid, A. 1992. Muscle spasms during pre-slaughter $\mathrm{CO}_{2}$ anaesthesia in pigs. Ethical considerations. Fleischwirtschaft 6, 1519-1522. 
Gentle, M. and Tilston, V. 2000. Nociceptors in the legs of poultry: Implications for potential pain in preslaughter shackling. Animal Welfare 9, 227-236.

Gerritzen, M.A. and Hindle, V.A. 2009. Indicatoren voor bewusteloosheid. Retrieved on 1 November 2013 from http://edepot.wur.nl/12436.

Gerritzen, M., Lambooij, B., Reimert, H., Stegeman, A., Spruijt, B. 2004. On-farm euthanasia of broiler chickens: effects of different gas mixtures on behaviour and brain activity. Poultry Science 83, 1294-1301.

Gerritzen, M., Reimert, H., Hindle, V., Verhoeven, M., Veerkamp, W. 2013. Multistage carbon dioxide gas stunning of broilers. Poultry Science 92, 41-50.

Gibson, T.J., Johnson, C.B., Stafford, K.J., Mitchinsont, S.L. and Mellor, D.J. 2007. Validation of the acute electroencephalographs responses of calves to noxious stimulus with scoop dehorning. New Zealand Veterinary Journal 55, 152-157.

Gibson, T., Johnson, C., Murrell, J., Chambers, J., Stafford, K., Mellor, D. 2009a. Components of electroencephalographic responses to slaughter in halothaneanaesthetised calves: Effects of cutting neck tissues compared with major blood vessels. New Zealand Veterinary Journal 57, 84-89.

Gibson, T., Johnson, C., Murrell, J., Hulls, C., Mitchinson, S., Stafford, K., Johnstone, A., Mellor, D. 2009b. Electroencephalographic responses of halothane-anaesthetised calves to slaughter by ventral-neck incision without prior stunning. New Zealand Veterinary Journal 57, 77-83.

Gibson, T., Johnson, C., Murrell, J., Mitchinson, S., Stafford, K., Mellor, D. 2009c. Electroencephalographic responses to concussive non-penetrative captive-bolt stunning in halothane-anaesthetised calves. New Zealand Veterinary Journal 57, 90-95.

Gibson, T.J., Dadios, N. and Gregory, N.G. 2015. Effect of neck cut position on time to collapse in halal slaughtered cattle without stunning. Meat Science 110, 310-314.

Gouveia, K., Ferreira, P., da Costa, J., Vaz-Pires, P. and da Costa, P.M. 2009. Assessment of the efficiency of captive-bolt stunning in cattle and feasibility of associated behavioural signs. Animal Welfare 18, 171-175.

Grandin, T. 1992. Effect of genetics on handling and $\mathrm{CO}_{2}$ stunning of pigs. Meat focus international 7, 124-26.

Grandin, T. 1994. Euthanasia and Slaughter of Livestock. Journal of the American Veterinary Medical Association 204, 1354-1360. 
Grandin, T. 1998. The feasibility of using vocalization scoring as an indicator of poor welfare during cattle slaughter. Applied Animal Behaviour Science 56, 121-128.

Grandin, T. 2002. Return-to-sensibility problems after penetrating captive bolt stunning of cattle in commercial beef slaughter plants. Journal of the American Veterinary Medical Association 221, 1258-1261.

Grandin, T. 2010. Recommended animal handling guidelines \& audit guide: a systematic approach to animal welfare. Retrieved on October $5^{\text {th }} 2014$ from http://www. animalhandling.org/ht/d/sp/i/26752/pid/26752

Grandin, T. 2013. Making slaughterhouses more humane for cattle, pigs, and sheep. Annual Review of Animal Bioscience 1, 491-512.

Grandin, T. and Regenstein, J.M. 1994. Religious slaughter and animal welfare: a discussion for meat scientists. Meat Focus International 3, 115-123.

Grandin, T. and Smith, G.C. 2004. Animal Welfare and Humane slaughter. Retrieved on $1^{\text {st }}$ of March 2014 from http://www.grandin.com/references/humane.slaughter.html

Gregory, N. 1998. Physiology of stress, distress, stunning and slaughter. Animal Welfare and Meat Science. Wallingford, Oxfordshire, England: CABI Publishing.

Gregory, N. 2001. Profiles of currents during electrical stunning. Australian Veterinary Journal 79, 844-845.

Gregory, N.G. 2005a. Recent concerns about stunning and slaughter. Meat Science 70, 481-491.

Gregory, N. 2005b. Bowhunting deer. Animal Welfare 14, 111-116.

Gregory, N.G. 2007. Animal welfare and meat production. $2^{\text {nd }}$ edition. CAB International. Oxfordshire, UK.

Gregory, N.G. 2008a. Physiology and Behaviour of Animal Suffering. UFAW Animal Welfare Series. Blackwell Publishing, Oxford, UK.

Gregory, N.G. 2008b. Animal welfare at markets and during transport. Meat Science 80, 2-11.

Gregory, N. and Wotton, S. 1984. Sheep slaughtering procedures. II. Time to loss of brain responsiveness after exsanguination or cardiac arrest. British Veterinary Journal 140, 354-360.

Gregory, N. and Wotton, S. 1986. Effect of slaughter on the spontaneous and evoked activity of the brain. British Poultry Science 27, 195-205. 
Gregory, N. and Wotton, S. 1989. Effect of electrical stunning on somatosensory evoked potentials in chickens. British Veterinary Journal 145, 159-164.

Gregory, N.G. and Wotton, S.B. 1990. Effect of stunning on spontaneous physical activity and evoked activity in the brain. British Poultry Science 31, 215-220.

Gregory, N. and Shaw, F. 2000. Penetrating captive bolt stunning and exsanguination of cattle in abattoirs. Journal of Applied Animal Welfare Science 3, 215-230.

Gregory, N., Shaw, F., Whitford, J. and Patterson-Kane, J. 2006. Prevalence of ballooning of the severed carotid arteries at slaughter in cattle, calves and sheep. Meat Science 74, 655-657.

Gregory, N.G., Lee, C.J. and Widdicombe, J.P. 2007. Depth of concussion in cattle shot by penetrating captive bolt. Meat Science 77, 499-503.

Gregory, N.G., von Wenzlawowicz, M., Alam, R.M., Anil, H.M., Yeşildere, T. and SilvaFletcher, A. 2008. False aneurysms in carotid arteries of cattle and water buffalo during shechita and halal slaughter. Meat Science 79, 285-288.

Gregory, N., Spence, J., Mason, C., Tinarwo, A. and Heasman, L. 2009a. Effectiveness of poll stunning water buffalo with captive bolt guns. Meat Science 81, 178-182.

Gregory, N., Wenzlawowicz, M. and Von Holleben, K. 2009b. Blood in the respiratory tract during slaughter with and without stunning in cattle. Meat Science 82, 13-16.

Gregory, N., Fielding, H., Von Wenzlawowicz, M. and Von Holleben, K. 2010. Time to collapse following slaughter without stunning in cattle. Meat Science 85, 66-69.

Gregory, N., Schuster, P., Mirabito, L., Kolesar, R. and McManus, T. 2012a. Arrested blood flow during false aneurysm formation in the carotid arteries of cattle slaughtered with and without stunning. Meat Science 90, 368-372.

Gregory, N., von Wenzlawowicz, M., von Holleben, K., Fielding, H., Gibson, T., Mirabito, L. and Kolesar, R. 2012b. Complications during shechita and halal slaughter without stunning in cattle. Animal Welfare 21, 81-86.

Grillner, S., Wallén, P., Saitoh, K., Kozlov, A. and Robertson, B. 2008. Neural bases of goal-directed locomotion in vertebrates--an overview. Brain Research Reviews 57, 2-12.

Grover, V.K. and Bharti, N. 2008. Measuring depth of aneasthesia - an overview on the currently avaiable monitoring systems. Retrieved on 15 November 2013 from http:// www.theiaforum.org/Article_Folder/measuring-depth-of-anaesthesia-availablemonitoring-systems.pdf. 
Guntheroth, W. and Kawabori, I. 1975. Hypoxic apnea and gasping. Journal of Clinical Investigation 56, 1371-1377.

Gutierrez, G., Reines, H.D. and Wulf-Gutierrez, M.E. 2004. Clinical review: hemorrhagic shock. Critical Care London 8, 373-381.

GWvD, 1992. Gezondheids-en welzijnswet voor dieren. Ministerie van landbouw, Natuurbeheer en Visserij. The Hague, The Netherlands.

$\mathrm{H}$

Hansen, A.J. 1985. Effect of anoxia on ion distribution in the brain. Physiological Reviews 65, 101-148.

Harfeld, J. 2010. Husbandry to industry: Animal agriculture, ethics and public policy. Between the Species 13, 132-162.

Hari, R., Portin, K., Kettenmann, B., Jousmäki, V., Kobal, G. 1997. Right-hemisphere preponderance of responses to painful $\mathrm{CO}_{2}$ stimulation of the human nasal mucosa. Pain 72, 145-51.

Hartmann, H., Rindermann, G., Siegling-Vlitakis, C., Arndt, G., Wolf, K. and Fries, R. 2010. Relationship between the response to the corneal reflex (depth of narcosis) and specific parameters in the slaughter blood of pigs narcotised with CO 2. Animal Welfare 19, 515-522.

Hartung, J., Nowak, B., Waldmann, K., Ellerbrock, S. 2002. $\mathrm{CO}_{2}$-stunning of slaughter pigs: effects on EEG, catecholamines and clinical reflexes. Deutsche tierarztliche Wochenschrift 109, 135-9.

Hazem, A.S., Grob, R. and Schulze, W. 1977. Objektivierung von Schmerz und Bewusstsein im Rahmen der konventionellen und rituellen Schlachtung von Wiederkäuern. Abschlussbericht über den Forschungsauftrag 108.

Hemsworth, P.H., Fisher, A.D., Mellor, D.J. and Johnson, C.B. 2009. A Scientific comment on the Welfare of Sheep Slaughtered without Stunning. Retrieved on 14 October 2013 from http://www.google.nl/url? sa=t\&rct=j\&q=\&esrc=s\&frm =1\&source=web\&cd=1\&v ed=0CCAQFjAA\&url=http $\% 3 \mathrm{~A} \% 2 \mathrm{~F} \% 2 \mathrm{Fwww}$.australiananimalwelfare.com.au\%2Fapp $\% 2$ Fwebroot $\% 2$ Ffiles $\% 2$ Fupload $\% 2$ Ffiles $\% 2$ Fwelfare-sheep-slaughter. pdf\&ei=LO7hU4vIDYbcOvW_gKAJ\&usg=AFQjCNEq52FDxL5rrarLsM311_ pF42qByw\&bvm=bv.72197243,d.ZWU.

Hoenderken, R. 1978. Electrical stunning of pigs for slaughter (PhD dissertation). Utrecht University, The Netherlands. 
Hoenderken, R. 1983. Electrical and carbon dioxide stunning of pigs for slaughter. In Stunning of Animals for Slaughter. Boston, USA: Martinus Nijhoff Publishers.

Holst, S. 2002. Behaviour in pigs immersed into atmospheric air or different carbon dioxide concentrations. Danish Meat Research Institute. Internal report 02.7097295.

Johnson, C., Young, S., Taylor, P. 1994. Analysis of the frequency spectrum of the equine electroencephalogram during halothane anaesthesia. Research in Veterinary Science 56, 373-378.

Johnson, C.B., Gibson, T.J., Stafford, K.J. and Mellor, D.J. 2012. Pain perception at slaughter. Animal Welfare 21, 113-122.

Johnson, C., Mellor, D., Hemsworth, P., Fisher, A. 2015. A scientific comment on the welfare of domesticated ruminants slaughtered without stunning. New Zealand Veterinary Journal 63, 58-65.

$\mathrm{K}$

Kallweit, E., Ellendorf, F., Daly, C. and Smidt, D. 1989. Physiologic reactions during the slaughter of cattle and sheep with and without stunning. Deutsche Tierarztliche Wochenschrift 96, 89-92.

Klemm, W. 2011. Why does REM sleep occur? A wake-up hypothesis. Frontiers in systems neuroscience 5, 1-12.

Knudsen, S.K, 2005. A review of the criteria used to assess insensibility and death in hunted whales compared to other species. Veterinary Journal 169, 42-59.

Kooi, K.A., Tucker, R.P. and Marshall, R.E. 1978. Spontaneous electrical activity of the normal brain. In Fundamentals of electroencephalography (Baseman VA), pp. 49-67. Harper and Row Publishers Inc., Hagerstown, MD, USA.

Kroeger, D., Florea, B. and Amzica, F. 2013. Human brain activity patterns beyond the isoelectric line of extreme deep coma. PLoS One 8, 1-13.

$\mathrm{L}$

Lambooy, E. 1981. Some neural and physiological aspects of electrical and mechanical stunning in ruminants (PhD dissertation). The Netherlands.

Lambooij, E. 2004. Electrical stunning. In Encyclopedia of Meat Sciences (ed. DK Jensen, C Devine and M Dikeman), pp. 1342-1348. Elsevier, Oxford, United Kingdom. 
Lambooy, E. and Spanjaard, W. 1981. Effect of the shooting position on the stunning of calves by captive bolt. The Veterinary Record 109, 359-361.

Lambooij, E., Kloosterboer, R.J., Gerritzen, M.A., van de Vis, J.W. 2006. Assessment of electrical stunning in fresh water of African Catfish (Clarias gariepinus) and chilling in ice water for loss of consciousness and sensibility. Aquaculture 254, 388-395.

Lambooij, E., van der Werf, J., Reimert, H. and Hindle, V. 2012. Restraining and neck cutting or stunning and neck cutting of veal calves. Meat Science 91, 22-28.

Laureys, S. 2005. Science and society: Death, unconsciousness and the brain. Nature Reviews Neuroscience 6, 899-909.

Laureys, S. and Tononi, G. 2009. The neurology of consciousness: cognitive neuroscience and neuropathology. $1^{\text {st }}$ edition. Academic Press, London, UK.

Levitis, D.A., Lidicker Jr, W.Z. and Freund, G. 2009. Behavioural biologists do not agree on what constitutes behaviour. Animal Behaviour 78, 103-110.

Limon, G., Guitian, J. and Gregory, N.G. 2010. An evaluation of the humaneness of puntilla in cattle. Meat Science 84, 352-355.

Lin, J. 2000. Physiological review article: Brain structures and mechanisms involved in the control of cortical activation and wakefulness, with emphasis on the posterior hypothalamus and histaminergic neurons. Sleep Medicine Reviews 4, 471-503.

Liu, G.T. and Ronthal, M. 1992. Reflex blink to visual threat. Journal of NeuroOphthalmology 12, 47-56.

Llonch, P., Andaluz, A., Rodríguez, P., Dalmau, A., Jensen, E.W., Manteca, X. and Velarde, A. 2011. Assessment of consciousness during propofol anaesthesia in pigs. Veterinary Record, 169, 1-2.

Llonch, P., Rodríguez, P., Velarde, A., de Lima, V.A., Dalmau, A. 2012. Aversion to the inhalation of nitrogen and carbon dioxide mixtures compared to high concentrations of carbon dioxide for stunning rabbits. Animal Welfare 21, 123-9.

Llonch, P., Rodríguez, P., Jospin, M., Dalmau, A., Manteca, X. and Velarde, A. 2013. Assessment of unconsciousness in pigs during exposure to nitrogen and carbon dioxide mixtures. Animal 7, 492-498.

Lopes da Silva, F.H. 1982. The assessment of unconsciousness: general principles and practical aspects. In Stunning of animals for slaughter (ed Eikelenboom G), pp. 3-12. Martinus Nijhoff Publishers, Zeist, The Netherlands. 
Low, P., Panksepp, J., Reiss, D., Edelman, D. and Van Swinderen, B. 2012. The Cambridge declaration on consciousness. Retrieved on $14^{\text {th }}$ of September 2015 from http:// fcmconference.org/img/CambridgeDeclarationOnConsciousness.pdf

Lowe, J., Abeyesinghe, S., Demmers, T., Wathes, C. and McKeegan, D. 2007. A novel telemetric logging system for recording physiological signals in unrestrained animals. Computers and Electronics in Agriculture 57, 74-79.

Manning, H.L., Schwartzstein, R.M. 1995. Pathophysiology of dyspnea. New England Journal of Medicine 333, 1547-53.

Martin, J.E. 2015. Humane mechanical methods for killing poultry on-farm (PhD dissertation). University of Glasgow.

Martín-Cancho, M.F., Lima, J.R., Luis, L., Crisostomo, V., Ezquerra, L.J., Carrasco, M.S., Uson-Gargallo, J. 2003. Bispectral index, spectral edge frequency 95\%, and median frequency recorded for various concentrations of isoflurane and sevoflurane in pigs. American Journal of Veterinary Research 64, 866-873.

Martín-Cancho, M.F., Lima, J.R., Luis, L., Crisóstomo, V., López, M.A., Ezquerra, L.J., Carrasco-Jiménez, M.S., Usón-Gargallo, J. 2006. Bispectral index, spectral edge frequency 95\% and median frequency recorded at varying desflurane concentrations in pigs. Research in Veterinary Science 81, 373-381.

Martoft, L., Jensen, E.W., Rodríguez, B.E., Jorgensen, P.F., Forslid, A. and Pedersen, H.D. 2001. Middle-latency auditory evoked potentials during induction of thiopentone anaesthesia in pigs. Laboratory Animal 35, 353-363.

Martoft, L., Lomholt, L., Kolthoff, C., Rodriguez, B.E., Jensen, E.W., Jørgensen, P.F. 2002. Effects of $\mathrm{CO}_{2}$ anaesthesia on central nervous system activity in swine. Laboratory Animals 36, $115-26$.

Marzin, V., Collobert, J,F., Jaunet, S. and Marrec, L. 2008. Measure of efficiency and quality of stunning by penetrating captive bolt in beef cattle. Revue de Médecine Vétérinaire 159, 423-430.

McKeegan, D.E.F., McIntyre, J., Demmers, T.G.M., Wathes, C.M., Jones, R.B. 2006. Behavioural responses of broiler chickens during acute exposure to gaseous stimulation. Applied Animal Behavioural Science 99, 271-286.

McKeegan, D.E.F., McIntryre, J.A., Demmers, T.G.M., Lowe, J.C., Wathes, C.M., van den Broek, P.L.C., Coenen, A.M.L. and Gentle, M.J. 2007. Physiological and behavioural responses of broilers to controlled atmosphere stunning: Implications for welfare. Animal Welfare 16, 409-426. 
McKeegan, D., Sandercock, D., Gerritzen, M. 2013a. Physiological responses to low atmospheric pressure stunning and the implications for welfare. Poultry Science 92, 858-868.

McKeegan, D., Reimert, H., Hindle, V., Boulcott, P., Sparrey, J., Wathes, C., Demmers, T. and Gerritzen, M. 2013b. Physiological and behavioural responses of poultry exposed to gas-filled high expansion foam. Poultry science 92, 1145-1154.

Mellor, D., Littin, K. 2004. Using science to support ethical decisions promoting humane livestock slaughter and vertebrate pest control. Animal Welfare 13, 127-132.

Mellor, D., Gibson, T., Johnson, C. 2009. A re-evaluation of the need to stun calves prior to slaughter by ventral-neck incision: an introductory review. New Zealand Veterinary Journal 57, 74-76.

Merskey, H.E. 1986. Classification of chronic pain: descriptions of chronic pain syndromes and definitions of pain terms. Pain Supplement 3, 1-224.

Meyer, R.E. 2015. Physiologic Measures of Animal Stress during Transitional States of Consciousness. Animals 5, 702-716.

Mitchell, R. and Berger, A. 1975. Neural regulation of respiration. The American Review of Respiratory Disease 111, 206-224.

Murrell, J.C. and Johnson, C.B. 2006. Neurophysiological techniques to assess pain in animals. Journal of Veterinary Pharmacology and Therapeutics 29, 325-335.

Nakyinsige, K., Man, Y., Aghwan, Z.A., Zulkifli, I., Meng, Y., Fatimah, A., Al-Kahtani, H. and Sazili, A. 2013. Stunning and animal welfare from Islamic and scientific perspectives. Meat Science 95, 352-361.

Nangeroni, L. and Kennett, P. 1963. An electroencephalographic study of the effect of shechita slaughter on cortical function in ruminants. Ithaca, Ithaca University.

Newhook, J.C., Blackmore, D.K. 1982a. Electroencephalographic studies of stunning and slaughter of sheep and calves: Part 1-The onset of permanent insensibility in sheep during slaughter. Meat Science 6, 221-233.

Newhook, J.C. and Blackmore, D.K. 1982b. Electroencephalographic studies of stunning and slaughter of sheep and calves-part 2: The onset of permanent insensibility in calves during slaughter. Meat Science 6, 295-300.

Niedermeyer, E, Schomer, D.L. and Da Silva, F.H. 2011. Niedermeyer's electroencephalography: Basic principles, clinical applications, and related fields. Wolters Kluwer Health, Philadelphia, United States of America. 
Nowak, B., Mueffling, T., Hartung, J. 2007. Effect of different carbon dioxide concentrations and exposure times in stunning of slaughter pigs: Impact on animal welfare and meat quality. Meat Science 75, 290-8.

$\mathrm{O}$

O’Connor, A.M., Dzikamunhenga, R.S., Totton, S., Glanville, J., and Wood, H. 2015. Review of the main welfare risks related to electrical stunning of small ruminants (ovine and caprine species). Veterinary Diagnostic and Production Animal Medicine Reports. Paper 3.

Parotat, S., von Holleben, K., Arnold, S., Troeger, K. and Luecker, E. 2015. Hot-water spraying is a sensitive test for signs of life before dressing and scalding in pig abattoirs with carbon dioxide $\left(\mathrm{CO}_{2}\right)$ stunning. Animal 10, 326-332.

Parshall, M.B., Schwartzstein, R.M., Adams, L., Banzett, R.B., Manning, H.L., Bourbeau, J., et al. 2012. An official American Thoracic Society statement: update on the mechanisms, assessment, and management of dyspnea. American Journal of Respiratory and Critical Care Medicine 185, 435-452.

Petty, D., Hattingh, J., Ganhao, M. and Bezuidenhout, L. 1994. Factors which affect blood variables of slaughtered cattle. Journal of the South African Veterinary Association 65, 41-45.

Prinz, S. 2009. Waterbath stunning of chickens. Effects of electrical parameters on the

electroencephalogram and physical reflexes of broilers ( $\mathrm{PhD}$ dissertation). Radboud University. The Netherlands.

$\mathbf{R}$

Raj, A.B.M. 1999. Behaviour of pigs exposed to mixtures of gases and the time required to stun and kill them: Welfare implications. Veterinary Record 144, 165-168.

Raj, A. 2006. Recent developments in stunning and slaughter of poultry. World's Poultry Science Journal 62, 467-84.

Raj, A. and Gregory, N. 1990 Effect of rate of induction of carbon dioxide anaesthesia on the time of onset of unconsciousness and convulsions. Research in Veterinary Science. 49, 360-63.

Raj, M. and Gregory, N. 1993. Time to loss of somatosensory evoked potentials and onset of changes in the spontaneous electroencephalogram of turkeys during gas stunning. Veterinary Record 133, 318-320. 
Raj, A. and Gregory, N. 1995. Welfare implications of the gas stunning of pigs 1 . Determination of aversion to the initial inhalation of carbon dioxide or argon. Animal Welfare 4:273-80.

Raj, A. and Gregory, N. 1996. Welfare implications of the gas stunning of pigs 2. Stress of induction of anaesthesia. Animal Welfare 5, 71-78.

Raj, A. and O'Callaghan, M. 2004. Effects of electrical water bath stunning current frequencies on the spontaneous electroencephalogram and somatosensory evoked potentials in hens. British Poultry Science 45, 230-236.

Raj, A.B.M, Gregory, N.G. and Wotton, S.B. 1991. Changes in the somatosensory evoked potentials and spontaneous electroencephalogram of hens during stunning in argoninduced anoxia. British Veterinary Journal 147, 322-330.

Raj, A., Wotton, S. and Gregory, N. 1992. Changes in the somatosensory evoked potentials and spontaneous electroencephalogram of hens during stunning with a carbon dioxide and argon mixture. British Veterinary Journal 148, 147-156.

Raj, A., Johnson, S., Wotton, S. and McInstry, J. 1997. Welfare implications of gas stunning pigs: 3 . the time toloss of somatosensory evoked potential and spontaneous electrocorticogram of pigs during exposure to gases. The Veterinary Journal 153, 329-339.

Raj, A., O'Callaghan, M. and Knowles, T. 2006. The effects of amount and frequency of alternating current used in water bath stunning and of slaughter methods on electroencephalograms in broilers. Animal Welfare 15, 7-18.

RDA, 2015. One Health, een afwegingskader voor beleidsbeslissingen.Retrieved on 265-2016 from http://www.rda.nl/home/files/2016/Zienswijze-One-Health.pdf

Ring, C., Erhard, W., Kraft, H., Schmid, A., Weinmann, M., Berner, H. and Unshelm, J. 1988. Zur Betäubung von Schlachtschweinen mittels $\mathrm{CO}_{2}$. Fleischwirtsch 68, 1304-1307.

Rodríguez, P., Dalmau, A., Ruiz-de-la-Torre, J.L., Manteca, X., Jensen, E.W., Rodríguez, B., Litvan, H. and Velarde, A. 2008. Assessment of unconsciousness during carbon dioxide stunning in pigs. Animal Welfare 17, 341-349.

Rodriguez, P., Velarde, A., Dalmau, A., Llonch, P. 2012. Assessment of unconsciousness during slaughter without stunning in lambs. Animal Welfare 21, 75-80.

Rollin, B. 2004. Annual meeting keynote address: Animal agriculture and emerging social ethics for animals. Journal of Animal Sciences 82, 955-964.

Roos, J. and Koopmans, S. 1936. The value of he eye-reflex in animals submitted to the so-called electrical stunning. Veterinary Journal 92, 127-137. 
Rosen, S. 2004. Physiological insights into Shechita. Veterinary Record 154, 759-765.

Rubin, M. and Safdieh, J.E. 2007. Netter's concise neuroanatomy. Saunders, Elsevier. Philadelphia, United States of America.

Russow, L.M. 1990. Ethical theory and the moral status of animals. The Hastings Center Report 20.

San-Juan, D., Chiappa, K.H., Cole, A.J. 2010. Propofol and the electroencephalogram. Clinical Neurophysiology: Official Journal of the International Federation of Clinical Neurophysiology 121, 998-1006.

Sandercock, D.A., Auckburally, A., Flaherty, D., Sandilands, V. and McKeegan, D.E.F. 2014. Avian reflex and electroencephalogram responses in different states of consciousness. Physiology \& Behaviour 133, 252-259.

Sandøe, P. and Christiansen, S.B. 2008. Ethics of animal use. Blackwell Publishing.

Savenije, B., Lambooij, E., Gerritzen, M. and Korf, J. 2002. Development of brain damage as measured by brain impedance recordings, and changes in heart rate, and blood pressure induced by different stunning and killing methods. Poultry Science 81, 572-578.

Schneider, G. and Sebel, P.S. 1997. Monitoring depth of anaesthesia. European Journal of Anaesthesiology 14, 21-28.

Schomer, D.L., Da Silva, F.L. 2012. Niedermeyer's electroencephalography: Basic principles, clinical applications, and related fields. Wolters Kluwer Health.

Schulze, W., Schultze-Petzold, H., Hazem, A. and Gross, R. 1978. Experiments for the objectification of pain and consciousness during conventional (captive bolt stunning) and religiously mandated ("ritual cutting") slaughter procedures for sheep and calves. Deutsche Tierarztliche Wochenschrift 85, 62-66.

Schwender, D., Daunderer, M., Mulzer, S., Klasing, S., Finsterer, U., Peter, K. 1996. Spectral edge frequency of the electroencephalogram to monitor" depth" of anaesthesia with isoflurane or propofol. British Journal of Anaesthesia 77, 179-184.

Serrats, A., Parker, S. and Merino-Canas, A. 1976. The blink reflex in coma and after recovery from coma. Acta Neurochirurgica 34, 79-97.

Seth, A.K., Baars, B.J., Edelman, D.B. 2005. Criteria for consciousness in humans and other mammals. Consciousness and Cognition 14, 119-139. 
Shaw, N.A. 2002. The neurophysiology of concussion. Progress in Neurobiology 67, 281-344.

Shields, S.J. and Raj, A. 2010. A critical review of electrical water-bath stun systems for poultry slaughter and recent developments in alternative technologies. Journal of Applied Animal Welfare Science 13, 281-299.

Siesjö, B.K. 1972. The regulation of cerebrospinal fluid pH. Kidney International 1, 360-74.

Sleigh, J., Donovan, J. 1999. Comparison of bispectral index, 95\% spectral edge frequency and approximate entropy of the EEG, with changes in heart rate variability during induction of general anaesthesia. British Journal of Anaesthesia 82, 666-671.

Sparrey, J. and Kettlewell, P. 1994. Shackling of poultry: is it a welfare problem? World's Poultry Science Journal 50, 167-176.

Sparrey, J.M. and Wotton, S.B. 1997. The design of pig stunning tong electrodes - A review. Meat Science 47, 125-133.

Staatscourant. 2012. Convenant onbedwelmd slachten volgens religieuze riten. Retrieved on 13 January 2015 from http://www.rijksoverheid.nl/documenten-en-publicaties/ convenanten/2012/06/05/convenant-onbedwelmd-slachten-volgens-religieuze-riten. html.

St John, W.M. 2009. Noeud vital for breathing in the brainstem: gasping-yes, eupnoeadoubtful. Philosophical Transactions of the Royal Society B: Biological Sciences 364, 2625 2633.

Stark, R., Gambles, S., Lewis, J. 1981. Methods to assess breathlessness in healthy subjects: a critical evaluation and application to analyse the acute effects of diazepam and promethazine on breathlessness induced by exercise or by exposure to raised levels of carbon dioxide. Clinical Science 61, 429-39.

Tagawa, M., Okano, S., Sako, T., Orima, H. and Steffey, E.P. 1994. Effect of change in body position on cardiopulmonary function and plasma cortisol in cattle. The Journal of veterinary medical science/the Japanese Society of Veterinary Science 56, 131-134.

Teplan, M. 2002. Fundamentals of EEG measurement. Measurement Science Review 2, 1-17.

Terlouw, E., Arnould, C., Auperin, B., Berri, C., Le Bihan-Duval, E., Deiss, V. 2008. Preslaughter conditions, animal stress and welfare: current status and possible future research. Animal 10, 1501-1517. 
Terlouw, E.M.C, Bourguet, C. and Deiss, V. 2016a. Consciousness, unconsciousness and death in the context of slaughter. Part 1. Neurobiological mechanisms underlying stunning and killing. Meat Science 118, 133-146.

Terlouw, E.M.C, Bourguet, C. and Deiss, V. 2016b. Consciousness, unconsciousness and death in the context of slaughter. Part 2. Evaluation methods. Meat Science 118, 147-156.

Tidswell, S., Blackmore, D. and Newhook, J. 1987. Slaughter methods: Electroencephalographs (EEG) studies on spinal cord section, decapitation and gross trauma of the brain in lambs. New Zealand Veterinary Journal 35, 46-49.

Tolo, E., Christensen, L., Martoft, L., Forslid, A. 2010. $\mathrm{CO}_{2}$-stunning in pigs. Animal Welfare 19, 369-73.

Troeger, K. 1991. Slaughtering: animal protection and meat quality. Current practiceWhat needs to be done? Fleischwirtschaft 71, 298-302.

Upton, R.N., Ludbrook, G.L. 1999. A model of the kinetics and dynamics of induction of anaesthesia in sheep: variable estimation for thiopental and comparison with propofol. British Journal of Anaesthesia 82, 890-899.

Upton, R.N., Martinez, A.M., Grant, C. 2009. Comparison of the sedative properties of CNS 7056, midazolam, and propofol in sheep. British Journal of Anaesthesia 103, 848-851.

Van der Burg, W., and van Willigenburg, T. 1998. Reflective equilibrium: Essays in honour of Robert Heeger. Springer Science \& Business Media.

Van Liere, E.J. and Stickney, J.C. 1963. Hypoxia. The University of Chicago Press, 1-348. Retrieved on May $18^{\text {th }} 2016$ from http://www.anesthesiaweb.org/images/hypoxia/LSH1963.pdf

van Zanden, J. and Koolmees, P. 2003. Het doden van dieren: historische aspecten.

Velarde, A., Gispert, M., Faucitano, L., Manteca, X., Diestre, A. 2000. The effect of stunning method on the incidence of PSE meat and haemorrhages in pork carcasses. Meat Science 55, 309-14.

Velarde, A., Ruiz-de-la-Torre, J.L., Roselló, C., Fàbrega, E., Diestre, A. and Manteca, X. 2002. Assessment of return to consciousness after electrical stunning in lambs. Animal Welfare 11, 333-341. 
Velarde, A., Cruz, J., Gispert, M., Carrión, D., Ruiz de la Torre, J.L., Diestre, A. 2007. Aversion to carbon dioxide stunning in pigs: effect of carbon dioxide concentration and halothane genotype. Animal Welfare 16, 513-22.

Verhoeven, M., Gerritzen, M., Hellebrekers, L. and Kemp, B. 2015a. Indicators used in livestock to assess unconsciousness after stunning: a review. Animal 9, 320-330.

Verhoeven, M., Gerritzen, M., Kluivers-Poodt, M., Hellebrekers, L., Kemp, B. 2015b. Validation of behavioural indicators used to assess unconsciousness in sheep. Research in Veterinary Science 101, 144-53.

Verhoeven, M., Gerritzen, M., Hellebrekers, L. and Kemp, B. 2016a. Validation of indicators used to assess unconsciousness in veal calves at slaughter. Animal x, 1-9.

Verhoeven, M., Gerritzen, M., Velarde, A., Hellebrekers, L. and Kemp, B. 2016b. Time to loss of consciousness and its relation to behaviour in slaughter pigs during stunning with 80 or 95\% Carbon Dioxide. Frontiers in Veterinary Science 3, x-x.

Vimini, R., Field, R., Riley, M. and Varnell, T. 1983. Effect of delayed bleeding after captive bolt stunning on heart activity and blood removal in beef cattle. Journal of Animal Science 57, 628-631.

Vogel, K., Badtram, G., Claus, J., Grandin, T., Turpin, S., Weyker, R. and Voogd, E. 2011. Head-only followed by cardiac arrest electrical stunning is an effective alternative to head-only electrical stunning in pigs. Journal of Animal Science 89, 1412-1418.

von Holleben, K., von Wenzlawowicz, M., Gregory, N., Anil, H., Velarde, A., Rodríguez, P., Cenci Goga, B., Catanese, B. and Lambooij, B. 2010. Report on good and adverse practices - Animal welfare concerns in relation to slaughter practices from the viewpoint of veterinary sciences. Retrieved on 6 October 2012 from http://rytualny.pl/data/uploads/ pdf/dialrel-animal-welfare-during-slaughter-report-2010.pdf.

von Wenzlawowicz, M., von Holleben, K. and Eser, E. 2012. Identifying reasons for stun failures in slaughterhouses for cattle and pigs: a field study. Animal Welfare 21, 51-60.

W

Warren, M.A. 1997. Moral status: Obligations to persons and other living things. Clarendon Press.

Warriss, P. 1984. Exsanguination of animals at slaughter and the residual blood content of meat. The Veterinary Record 115, 292-295.

Wijdicks, E.F. 2001. The diagnosis of brain death. New England Journal of Medicine 344, 1215-1221. 
Wilkins, L., Gregory, N., Wotton, S. and Parkman, I. 1998. Effectiveness of electrical stunning applied using a variety of waveform-frequencycombinations and consequences for carcase quality in broiler chickens. British Poultry Science 39, 511-518.

Williams, V. 2004. The use of carbon dioxide for euthanasia and anaesthesia. Surveillance 31, 28-30.

Woolley, S.C., Borthwick, F. and Gentle, M. 1986. Tissue resistivities and current pathways and their importance in pre-slaughter stunning of chickens. British Poultry Science 27, 301-306.

Wotton, S. and Sparrey, J. 2002. Stunning and slaughter of ostriches. Meat Science 60, 389-394.

$\mathrm{Z}$

Zeller, W., Schatzmann, U., Imhof, A. 1987. Kohlendioxid Betaubung von Schlachttieren. Fleischwirtschaft 67, 1519-22.

Zeman, A. 2001. Consciousness. Brain 124, 1263-1289.

Zeman, A. 2005. What in the world is consciousness? Progressive Brain Research 150, $1-10$.

Zulkifli, I., Goh, Y., Norbaiyah, B., Sazili, A., Lotfi, M., Soleimani, A., Small, A. 2014. Changes in blood parameters and electroencephalogram of cattle as affected by different stunning and slaughter methods in cattle. Animal Production Science 54, 187-193. 
REFERENCES | 171 


\section{LIST OF PUBLICATIONS}

\section{Refereed scientific journals}

Van Leeuwen, J.J.J., Verhoeven, M.T.W., van der Heden-van Noort, I., Kranenbarg, S., Kemp, B., and Soede, N. 2012. Split-weaning before Altrenogest synchronization of multiparous sows alters follicular development and reduces embryo survival. Reproduction in Domestic Animals 47: 530-536.

Gerritzen, M.A., Reimert, H.G.M., Hindle, V.A., Verhoeven, M.T.W., and Veerkamp, W.B. 2013. Multistage carbon dioxide gas stunning of broilers. Poultry Science 92, 41-50.

Gerritzen, M.A., Reimert, H.G.M., Lourens, A., Bracke, M.B.M., and Verhoeven, M.T.W. 2013. Killing wild geese with carbon dioxide or a mixture of carbon dioxide and argon. Animal Welfare 22, 5-12.

Lambooij, E., Reimert, H.G.M., Verhoeven, M.T.W., and Hindle, V.A. 2014. Cone restraining and head-only electrical stunning in broilers: Effects on physiological responses and meat quality. Poultry Science 93, 512-518.

Verhoeven, M.T.W., Gerritzen, M.A., Hellebrekers, L.J., and Kemp, B. (2015a). Indicators to assess unconsciousness in livestock after stunning: a review. Animal 9, 320-330.

Verhoeven, M.T.W., Gerritzen, M.A., Kluivers-Poodt, M., Hellebrekers, L.J., and Kemp, B. 2015b. Validation of behavioural indicators used to assess unconsciousness in sheep. Research in Veterinary Science 101, 144-153.

Ellen, E.D., Peeters, K., Verhoeven, M.T.W., Gols, R., Harvey, J.A., Wade, M.J., Dicke, M., and Bijma, P. 2016. Direct and indirect genetic effects in life-history traits of flour beetles (Tribolium castaneum). 2016. Evolution 70, 207-217.

M.T.W. Verhoeven, M.A. Gerritzen, L.J. Hellebrekers, and B. Kemp. 2016. Validation of indicators used to assess unconsciousness in veal calves at slaughter. Animal x, 1-9.

Verhoeven, M., Gerritzen, M., Velarde, A., Hellebrekers, L. and Kemp, B. 2016. Time to loss of consciousness and its relation to behaviour in slaughter pigs during stunning with 80 or 95\% Carbon Dioxide. Frontiers in Veterinary Science 3. 


\section{CONFERENCE PROCEEDINGS AND ABSTRACTS}

Ellen, E.D., Verhoeven, M.T.W., Peeters, K., Gols, R., Wade, M.J., Dicke, M., and Bijma, P. 2012. Using Tribolium castaneum as a model organism to investigate social interactions among individuals. Proceedings of the $4^{\text {th }}$ International Conference on Quantitative Genetics, 17-22 June, Edinburgh, United Kingdom.

Gerritzen, M.A., Workel, L.D., Reimert, H.G.M., Lourens, A., Verhoeven, M.T.W., Bracke, M.B.M. 2012. Killing wild geese with $\mathrm{CO}_{2}$ and argon. Proceedings of the Minding Animals Conference, 4-6 July, Utrecht, the Netherlands.

Verhoeven, M.T.W., Gerritzen M.G., Peuscher, J., Hellebrekers, L.J., and Kemp, B. 2013. Assessing the EEG in pigs anaesthetised with propofol. Proceedings of the Benelux International Society for Applied Ethology (ISAE) Meeting, 10 October, Sterksel, The Netherlands.

Verhoeven, M.T.W., Gerritzen, M.A., Reimert H.G.M., Hellebrekers, L.J., and Kemp, B. 2013. Assessing unconsciousness to determine effective stunning in pigs stunned with $\mathrm{CO}_{2}$ (poster). Wageningen Institute of Animal Science (WIAS) Science Day, 28 February, Wageningen, the Netherlands.

Verhoeven, M.T.W., Gerritzen, M.A., Kluivers-Poodt, M., Hellebrekers, L.J., and Kemp, B. 2014. Validation of indicators used in sheep to assess consciousness. Proceedings of the WIAS Science Day, 30 April, Wageningen, the Netherlands.

Verhoeven, M.T.W., Peuscher, J., Gerritzen M.A., 2014. Measuring physiological parameters using non-invasive techniques in sheep (poster). Proceedings of the $4^{\text {th }}$ UFAW One-day conferences on 'Recent advances in animal welfare science', 26 June, York, United Kingdom.

Verhoeven, M.T.W., Gerritzen, M.A., Hellebrekers, L.J., and Kemp, B. 2014. Validation of indicators to assess consciousness in sheep. Proceedings of the $65^{\text {th }}$ Annual Meeting of the European Federation of Animal Science (EAAP), 25-29 August, Copenhagen, Denmark.

Verhoeven, M.T.W., Gerritzen, M.A., Hellebrekers, L.J., and Kemp, B. 2015. Validation of indicators used to assess unconsciousness in cattle at slaughter. Proceedings of the HSA International Symposium: Recent Advances II. 16-17 July, Zagreb, Croatia. 
Verhoeven, M.T.W., Gerritzen, M.A., Hellebrekers, L.J., and Kemp, B. 2015. Time to loss of consciousness and the relation with behaviour in fattening pigs. Proceedings of the Benelux International Society for Applied Ethology (ISAE) Meeting, 15 October, Geel, Belgium.

\section{OTHER PUBLICATIONS RELATED TO THIS THESIS}

Verhoeven, Merel. 2013. Waarom heeft stress invloed op de kwaliteit van vlees? KIJK Magazine 11, page 77.

\section{AWARDS AND TRAVEL GRANT}

Award for Best oral presentation by a young scientist in the sheep and goat session of the 65 ${ }^{\text {th }}$ EAAP congress, Copenhagen, Denmark, 25-29 August, 2014.

Award for Best oral presentation at the Benelux ISAE meeting, Geel, Belgium, 15 October, 2015.

Travel grant LEB Foundation for participation in the HSA International Symposium: Recent Advances II, Zagreb, Croatia, 16-17 July, 2015. 
LIST OF PUBLICATIONS | 175 


\section{ACKNOWLEDGEMENTS}

Ondanks dat veel mensen me kennen als een zelfstandige, assertieve dame, had ik dit proefschrift nooit kunnen schrijven zonder de hulp van zovelen. Hoewel ik vele mensen op verschillende manieren dankbaar ben, kwam ik er door de jaren heen wel achter hoezeer ik het getroffen heb met mijn begeleidingscommissie.

Marien, ruim 4 jaar geleden durfde je het aan om met mij dit promotietraject te starten. Door de kennis die je hebt van de sector en wegens het feit dat je die met mij wilde delen, heb je vele deuren geopend voor me. Ik heb een groot respect voor je als persoon, alsmede de manier waarop je je zowel in de wetenschap als de praktijk beweegt. Ik heb altijd dankbaar gebruik (misbruik?) gemaakt van jouw 'open-door-policy' en had me geen betere en prettigere dagelijks begeleider kunnen wensen. Ook voor jou is het vandaag extra feest, want je hebt je eerste aio afgeleverd $(:)$ Gefeliciteerd!

Ik kan me mijn $\mathrm{PhD}$ project echter ook niet voorstellen zonder jou Bas, mijn promotor. Ondanks dat mijn $\mathrm{PhD}$ onderwerp geen dagelijkse kost voor je was, wist je orde te scheppen in alle analyse mogelijkheden en creëerde je een duidelijke structuur in het proefschrift. Je suggesties en ideeën hebben elk manuscript een stuk duidelijker en overzichtelijker gemaakt. En hoewel ik een Lelystad-aio was, gaf je me toch een plek op je afdeling en maakte je me onderdeel van de 'ADP-familie'. Je hebt me vaak bemoedigende woorden toegesproken wanneer je doorhad dat ik dat even nodig had. Veel dank daarvoor!

Ludo, als externe begeleider was je wellicht minder betrokken bij mijn dagelijkse begeleiding, maar zeker niet minder betrokken bij het project. Ik heb je meerdere malen, zeker in de laatste weken, onmogelijke hoeveelheden papier toegestuurd met de vraag of je binnen korte tijd wilde reageren. Niet alleen haalde je die deadlines, je had de manuscripten ook nog eens zeer nauwkeurig doorgelezen. Ik ben je dan ook zeer dankbaar voor al je input. Daarnaast heb ik je interesse in mij als persoon altijd zeer op prijs gesteld, zeker toen het met mij minder goed ging. Ik hoop dan ook dat onze wegen elkaar in de toekomst nog eens zullen kruisen.

I am grateful to Dr. Dorothy McKeegan, Dr. Claudia Terlouw, Dr. Michael Marahrens and Prof. Dr Peter Groot Koerkamp for reading and assessing my thesis and for being a member of the examination committee during the public defence.

Bij het overgrote deel van de onderzoeken die ik heb uitgevoerd was jij, Jan, ook betrokken. Naast technisch onderlegd, ben je innovatief en creatief en zag je altijd mogelijkheden in 
plaats van obstakels. Door jouw handigheid met naald en draad droegen mijn schapen, varkens en koeien modieuze hesjes en halsters en werden er daarmee ook nog eens goede signalen opgepikt. Ik heb altijd met veel plezier met je samengewerkt! Henny, jij leerde me de kneepjes van het vak in de eerste jaren. Je tomeloze geduld, maar bovenal je interesse in mijn onderzoek en mij als persoon heb ik altijd ontzettend gewaardeerd. Vincent, bedankt voor je hulp op verschillende vlakken in mijn project. Naast de financiële man, was je ook praktisch betrokken. Door een beetje bloed liet jij je gelukkig niet afschrikken. Dank ook voor alle Engelse spelling controles, ik kreeg meteen minder opmerkingen van reviewers. Marije, wat een rots in de branding was jij op het einde. Wanneer ik met jou gespard had of ik je commentaar op mijn stukken las, had ik altijd weer het vertrouwen dat het goed kwam. De speciaal biertjes zal ik daarom niet vergeten als ik weer in Oslo ben! Bastiaan, ik was ontzettend blij dat ik jou in de beginfase van mijn aio-traject heb gevonden om te sparren over programmeren en Matlab. Helaas klikten Matlab en ik niet zo, maar aan jouw geduld en inzet heeft het zeker niet gelegen!

Een onmisbare pijler in dit onderzoek wordt gevormd door de mensen die verantwoordelijk zijn voor het reilen en zeilen op de proefaccomodaties en de verzorging van de dieren. In dit verband wil ik graag mijn dank uitspreken naar de medewerkers van de proefaccomodatie in Lelystad. Je hebt niet altijd voor het kiezen wie er in je project werken, maar ik kan iedereen een man al jij, Dirk, aanbevelen. Jouw kennis van de anesthesiologie, gecombineerd met je positieve instelling, hebben het altijd een feest gemaakt om met je te werken! Daarnaast wil ik graag de medewerkers van slachterij Beernink bedanken voor hun inzet. Extra dank gaat uit naar Gerard voor het openstellen van zijn slachterij en Paul voor het organisatorische gedeelte van het betreffende onderzoek. Antonio, thank you for providing me the opportunity to perform my final experiment abroad at facilities. It was a great pleasure to work with you and with your technicians Quim and Xenia. They rock!

Nanette en Lora: jullie zijn echt onmisbaar! Bedankt voor alle administratieve en praktische zaken die jullie voor me regelden. Ik kon altijd bij jullie terecht, ook voor een praatje (dat waren er nog heel wat). Ik gun elk bedrijf een secretariaat als dat van ADP en kan niet anders dan benadrukken dat het meer dan terecht is geweest dat jullie team van het jaar zijn geweest. Lora, ik heb genoten van mijn paardrijd uurtjes met Milou; tof dat je haar mij toevertrouwt.

In elk proefschrift zit inbreng van studenten verborgen en dat is in dit proefschrift niet anders. Samantha, dank voor het werk dat je hebt verzet tijdens je Master afstudeervak. Barneveld heeft hierdoor niet 1, maar 2 mensen met een bovengemiddelde kennis van het beoordelen van bewusteloosheid tijdens het slachtproces. 
Danny, bedankt voor je inzet met betrekking tot de statistische analyses. Ik heb je hulp en tomeloze geduld zeer gewaardeerd.

Dan zijn er diverse (ex) collega's die ik wil bedanken voor hulp en ondersteuning in vele vormen en op verschillende momenten. Allereerst al mijn collega's van de Afdeling Dierenwelzijn van Wageningen UR Livestock Research. Bedankt voor de leuke borrels en afdelingsuitjes. Sander, het was een feestje om deze met jou te organiseren! De volgende keer gaan we naar Ibiza toch? Kathalijne en Marion, kamergenoten in Lelystad, bedankt voor de gezelligheid op de kamer en jullie interesse in mijn onderzoek. Marion, extra dank voor het mee opzetten en je hulp tijdens het schapenexperiment.

Daarnaast zijn er ook de collega's van de afdeling Adaptatiefysiologie (ADP) van Wageningen Universiteit. Omdat ik voor mijn promotie al een jaar hier werkte, maakte dit het heel gemakkelijk terug te keren naar deze groep. Lieve Fleur, Monique, Bjorge, Rudie, Ger en Ilona: hoewel jullie tijdens mijn experimenten niet betrokken waren als OBP'ers, mocht ik altijd binnenlopen voor informatie, lab-spullen, protocollen of gewoon een praatje. Wat heb ik dat fijn gevonden! Ik vond het dan ook heel tof dat jullie op onze bruiloft waren! Dear Tropical Roomies, one can learn so much when interacting with people from foreign countries. I have enjoyed our parties, dinners and talks about cultural differences at work. For those who still need to defend, especially Junchai, best of luck, you can do it!

Ik heb mijn kantoor de afgelopen jaren mogen delen met een hoop toffe mensen: Maarten, Mariska en Moniek. Wat een fijne support groep waren jullie, op allerlei vlakken. Ik wens jullie heel veel succes met de laatste loodjes! Pieter, ik heb groot respect dat je je hart heb gevolgd. Je droom om fulltime met Ijslanders te kunnen werken gaat zeker een keer uitkomen! David, naast dat je een fijne kamergenoot was, heb je me uren tijd bespaard met je computer skills, veel dank daarvoor (-). Carla, je droge humor, interesse en luisterend oor waren soms zo welkom en maakten menig saai kantoor dag leuk $;$. Miss Renny, we hebben uren gewandeld, gepraat en je parate kennis uit de diergeneeskunde hoek is me vaak van pas gekomen. Ik wens je een fantastische bruiloft volgend jaar! Ik hoop het in de toekomst ook zo te treffen met mijn office mates, thnx!

Daarnaast heb ontzettend veel collega aio's die allen op hun eigen manier belangrijk zijn geweest, maar enkele wil ik toch apart naar voren halen: Nanda, je was mijn mede-mattie in Lelystad. Bedankt dat ik mocht spuien als de zaken niet liepen zoals ik het wilde, maar ook de gezellige borreluurtjes. Elske, wat was het tof om in York met je op te trekken, samen met Remco. Lydia, het kwam er wellicht minder vaak van dan we wilden, maar onze diners en breaks waren een zeer welkome afleiding! Heel veel succes met het 
uitbreiden van Ex-Equo. Zoals jij je ziel en zaligheid erin kunt leggen, weet ik zeker dat dat goed komt! Britt, cola-light mattie, je was er altijd voor in om even pauze te houden en ik heb genoten van onze breaks en chill-momentjes, ook buiten Zodiac, in Barneveld.

I have two colleagues in the area of stunning and slaughter that I wish to thank specifically. Jess, I have always been impressed with your work as a scientist, your knowledge, but also your personality. It was a pleasure learning from you those two weeks in Ayr, Scotland and above all, it was great to have a female friend in this area of research who could relate to my stories. Mariagrazia, never have I met such a kind and hospital person as yourself. You are a great person and scientist and I tremendously enjoyed our amazing holiday with you and Ferdinando in Naples. I hope we stay in touch, I value our friendship a lot!

I did not only focus on my PhD project the past four years, but I was also an active member in multiple committees. Being a member of the WAPS-Council and the WPC has taught me many things, but most of all has given me a lot of joy! Thnx to all committee members during those years.

Of course, there are a lot of people in and outside of Zodiac, close to me or a bit further away, that have made these past four years a great experience because we had fun working together, because we were in conferences or courses or just because we talked in the hallway. I may not have mentioned you specifically here, but thank you!

Als je niet kunt ontspannen in je vrije tijd of lol kunt maken met vrienden, dan is het schrijven van een proefschrift een stuk lastiger. Lieve vrienden, dank jullie wel voor jullie interesse in mijn onderzoek, de steuntjes in de rug en de nodige ontspanning die zo belangrijk is voor een aio. Zeker dit afgelopen jaar heb ik veel aan jullie steun gehad en ik voel me gezegend met zulke fijne mensen om me heen.

Daarnaast is er uiteraard ook 'het thuisfront' die je op wat voor manier dan ook helpt een proefschrift te schrijven. Jan $\dagger$, het voelt vreselijk raar dat jij er vandaag niet bij kunt zijn. Ik weet hoe trots je op me was en heb me altijd enorm gesteund gevoeld door je. Je zult nooit vergeten worden! Eef, jouw deur staat altijd open en je hebt me vanaf het begin af aan onderdeel van het gezin laten voelen, ik ben je daar zeer dankbaar voor! Lieve zwagers (Dennis en Marcel), schoonzussen (Aniek en Stevanie) en uiteraard Sophie: bedankt voor de gezelligheid aan tafel, met uitjes en bovenal jullie interesse!

Elza tsja, kouwe kant, maar zo voelt het niet hoor! Leuk dat je er vandaag bij kunt zijn! Dr. Floor, lieve zus, gelukkig was jij eerder klaar dan ik met promoveren (-). We hebben wat afgekibbeld in het verleden, maar ik ben blij dat naarmate we ouder worden we meer 
en meer raakvlakken krijgen! Thushara, broer(tje) lief: je mag dan wel ouder zijn dan ik, ik heb altijd de behoefte gevoeld je te bemoederen. Uiteindelijk bleek dit helemaal niet nodig. Je hebt het dik voor elkaar. Als jij achter die draaitafel staat, is dat een feest op zich. Ik ben dan ook blij dat zowel jij als Floor vandaag mijn steun op het podium zijn!

Ik ben ontzettend trots om de dochter van Jacques en Carlies, papa en mama, te zijn. Jullie liefde, steun en (financiële ;-)) zorg tijdens mijn opvoeding, studententijd en zelfs nu nog, vormden de basis voor dit proefschrift. Papa, bedankt dat je je creativiteit wilde wijden aan tekeningen voor presentaties en uiteraard de kaft van dit proefschrift. Mama, bedankt voor het altijd luisterend oor en de adviezen die je gaf, zowel zakelijk en privé.

Remco, dank voor je liefde en geduld, maar des te meer voor je kritiek en tegenspraak wanneer dit nodig was. De afgelopen jaren waren mooi, de komende jaren worden, als het aan mij ligt, nog mooier. 
ACKNOWLEDGEMENTS | 181 


\section{ABOUT THE AUTHOR}

Merel Theresa Willemijn Verhoeven was born in Breda, the Netherlands, in 1986. She completed her secondary education with honours (Gymnasium) in 2004 in Breda and hereafter started her BSc Animal Sciences in Wageningen. In 2008 she obtained this degree with a minor in Education and Competence studies after which she started her MSc Animal Sciences, also at Wageningen University, with a specialization

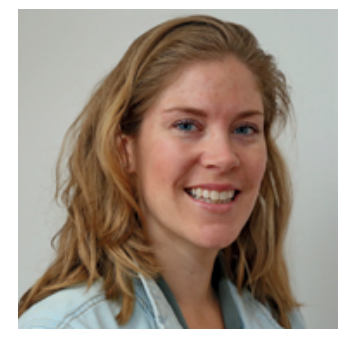
in 'Animal Health and Behaviour'. She conducted both a minor and major thesis at the Adaptation Physiology Group of Wageningen University. Merel's minor thesis focussed on assessing behavioural flexibility in chickens using clicker training. During her major thesis she looked at the effects of follicle size at ovulation on embryo development after Altrenogest treatment in sows. She conducted her internship (4 months) at the University of Alberta, Edmonton, Canada, where she assessed the effect of 'skip-a-heat' or Altrenogest treatment after weaning on reproduction performance in sows.

In 2010, Merel started working as a research assistant at the Adaptation Physiology Group, were she was involved in behavioural research, but also conducted a variety of lab analyses. In 2011 she started as a junior researcher at the Animal Welfare Department of Wageningen UR Livestock Research where she was involved in multiple projects regarding the health and welfare of livestock.

In 2012, Merel started her PhD at the Adaptation Physiology Group in collaboration with the department of Animal Welfare of Wageningen UR Livestock Research in Lelystad. During her PhD, she investigated different ways of assessing unconsciousness in livestock subjected to different stunning and slaughter methods. The results of this research are described in this thesis and have been published in a wide range of journals. After obtaining her $\mathrm{PhD}$ degree Merel aims to stay involved in areas related to animal health, welfare, physiology and nutrition. Merel can be contacted at: mtwverhoeven@ gmail.com. 
ABOUT THE AUTHOR | 183 


\section{TRAINING AND SUPERVISION PLAN}

\section{Completed Training and Supervision Plan ${ }^{1}$}

\section{The Basic Package}

3.0 ECTS

WIAS Introduction course

WIAS course on Philosophy of Science and Ethics

\section{International Conferences}

\subsection{ECTS}

$2^{\text {nd }}$ Minding Animals congress, Utrecht, the Netherlands

$8^{\text {th }}$ Measuring behaviour congress, Utrecht, the Netherlands

$3^{r d}$ UFAW congress, Barcelona, Spain

$3^{r d}$ International Summer School, Kulmbach, Germany

Benelux ISAE congress, Sterksel, the Netherlands

$4^{\text {th }}$ UFAW congress, York, United Kingdom

$2^{\text {nd }}$ HSA International Symposium, Zagreb, Croatia

ISAE Benelux congress, Geel, Belgium

\section{Seminars and Workshops}

\section{Presentations}


Theatre, ISAE Benelux congress, Geel, Belgium

Theatre, University of Naples “Federico II”, Naples, Italy 2015

Poster, $4^{\text {th }}$ UFAW congress, York, United Kingdom 2014

\section{In-depth Courses}

6.3 ECTS

UvA course 'Signal Analysis', Amsterdam, the Netherlands

WIAS course 'Statistics For the Life Sciences', Wageningen, the Netherlands

WIAS course 'Design of Experiments', Wageningen, the Netherlands

\section{Statutory Courses}

2.5 ECTS

Use of laboratory animals, Wageningen, the Netherlands

\section{Professional Skills Support Courses}

Course 'Basic project management', Wageningen, the Netherlands

Personal profile Insights Discovery, Lelystad, the Netherlands

WGS course 'Techniques for writing and presenting a scientific paper', Wageningen, the Netherlands

\section{Research Skills Training}

Preparing own research proposol: WIAS proposal

\section{Didactic Skills Training}

Assisting in MSc course 'Adaptation Physiology-2' 
Management Skills Training

8.5 ECTS

Secretary of the WIAS Associated PhD Students Council

2014-2015

Chair of the WIAS Associated PhD Students Council

2015-2016

Member of the Wageningen $\mathrm{PhD}$ Council

2014-2016

Organisation ' 5 th workshop on fundamental physiology and perinatal development in poultry'

2011

Organisation 'ISAE Benelux congress' 
Colophon

The research presented in this thesis was financed by the Dutch Ministery of Economic Affairs.

Cover: Jac Verhoeven, Breda, the Netherlands

Design\& Layout: Wendy Schoneveld, www.wenzid.nl

Printing: $\quad$ GVO Drukkers \& Vormgevers | Ponsen \& Looijen B.V., Ede, the Netherlands 

(2)

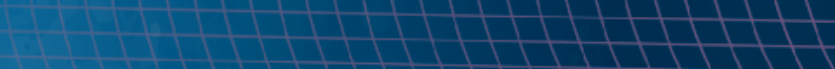

$-1210$

24 1 1 मे मे 24 D

(12) - -1 मे मे

-

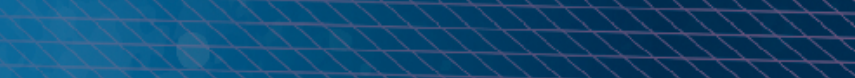

प्रे

प्रेशिले प्रेशे

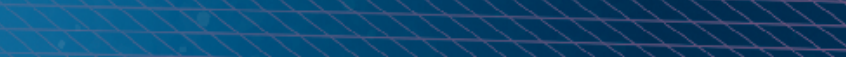

$2+2=0+2$

St

$\mathrm{x}$

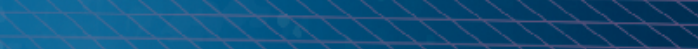

$\sqrt{\mathrm{P}}$

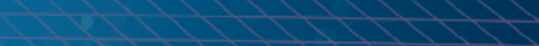

पर

(2)

40

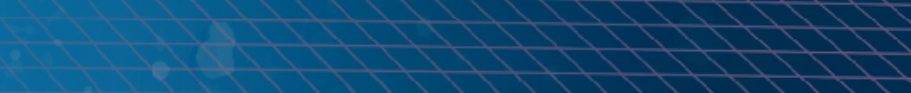

Q.

40

20

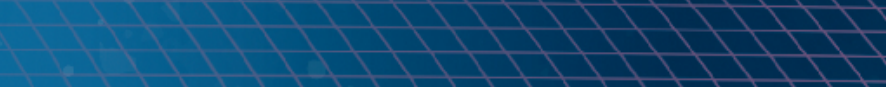

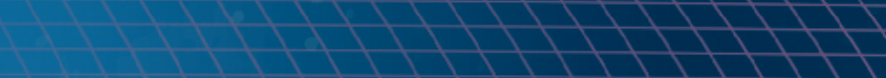

10.01

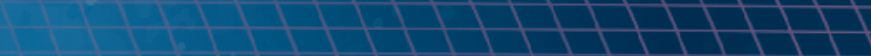

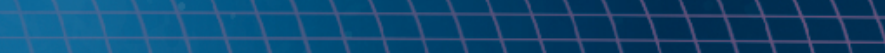

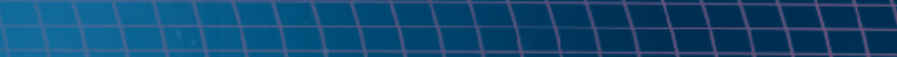

